\title{
Dementia, depression and forgetfulness : clinical studies of the early diagnosis and the differential diagnosis of dementia
}

Citation for published version (APA):

Verhey, F. R. J. (1993). Dementia, depression and forgetfulness : clinical studies of the early diagnosis and the differential diagnosis of dementia. [Doctoral Thesis, Maastricht University]. Datawyse / Universitaire Pers Maastricht. https://doi.org/10.26481/dis.19931027fv

Document status and date:

Published: 01/01/1993

DOI:

10.26481/dis.19931027fv

Document Version:

Publisher's PDF, also known as Version of record

Please check the document version of this publication:

- A submitted manuscript is the version of the article upon submission and before peer-review. There can be important differences between the submitted version and the official published version of record.

People interested in the research are advised to contact the author for the final version of the publication, or visit the DOI to the publisher's website.

- The final author version and the galley proof are versions of the publication after peer review.

- The final published version features the final layout of the paper including the volume, issue and page numbers.

Link to publication

\footnotetext{
General rights rights.

- You may freely distribute the URL identifying the publication in the public portal. please follow below link for the End User Agreement:

www.umlib.nl/taverne-license

Take down policy

If you believe that this document breaches copyright please contact us at:

repository@maastrichtuniversity.nl

providing details and we will investigate your claim.
}

Copyright and moral rights for the publications made accessible in the public portal are retained by the authors and/or other copyright owners and it is a condition of accessing publications that users recognise and abide by the legal requirements associated with these

- Users may download and print one copy of any publication from the public portal for the purpose of private study or research.

- You may not further distribute the material or use it for any profit-making activity or commercial gain

If the publication is distributed under the terms of Article 25fa of the Dutch Copyright Act, indicated by the "Taverne" license above, 


\title{
Dementia, depression and forgetfulness
}

\author{
Clinical studies \\ of the early diagnosis \\ and the differential diagnosis \\ of dementia
}


Photograph cover:

"Am Stamberger See", from left to right: A. Alzheimer, E. Kroepelin, R. Gaupp and F. Nissl

Printed by Datawyse Maastricht / krips Repro Meppel

CIP-GEGEVENS KONINKLIJKE BIBLIOTHEEK, DEN HAAG

Verhey, Franciscus Rochus Jozef

Dementia, depression and forgetfulness: dinical studies

of the earty and the differential diagnosis of

dementia / Franciscus Rochus Jozef Verhey. - Maastricht

Universitaire Pers Maastricht. - Ill.

Ook tekst in het Nederlands. - Proefschrift Maastricht. -

Met lit. opg.

ISBN 90-5278-097-8

NUGI 744

The financial support from Pfizer Nederland bv. (main sponsor) for the publication of this thesis is greatfully acknowledged:

The author whishes also to thank the following companies for their financial support: Boots Pharmaceuticals Nederland, Ethipharma Nederland-Sigma Tau company, Eli Lilly, Hoechst Pharma, Glaxo, Duphar Nederland, Organon International, Roche Nederland, Schering Nederland. 


\title{
Dementia, depression and forgetfulness
}

\author{
Clinical studies \\ of the early diagnosis \\ and the differential diagnosis \\ of dementia
}

\section{PROEFSCHRIFT}

ter verkrijging van de graad van doctor aan de

Rijksuniversiteit Limburg te Maastricht, op gezag van de Pro-Rector, Prof. dr. L. Boon, volgens het besluit van het College van Dekanen, in het openbaar te verdedigen op woensdag, 27 oktober 1993 om 16.00 uur

$$
\text { door }
$$

Franciscus Rochus Jozef Verhey

geboren op 9 november 1955 te Maastricht

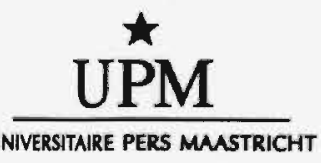


Promotores:

Prof. dr. J. Jolles

Prof. dr. H. M. van Praag

Beoordelingscommissie: Prof. dr. M. A. van den Hout (voorzitter)

Prof. dr. H. van Crevel (Universiteit van Amsterdam)

Prof. dr. J. A. Flendrig

Prof. dr. W. van Tilburg (Vrije Universiteit Amsterdam)

Prof. dr. J. Troost

The studies described in this thesis were carried out at the Department of Neuropsychology, Neuropsychiatry \& Psychobiology and the Academic Psychiatric Center, within the research programme 'Aging of Brain and Behavior' of the University of Limburg, Maastricht, The Netherlands and at the Department of Psychiatry, Academic Hospital of Maastricht. 


\section{Table of contents}

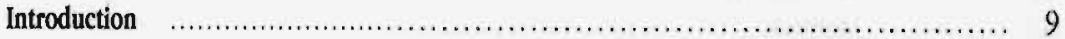

Rationale of the need for an early diagnosis of dementia $\ldots \ldots \ldots \ldots \ldots \ldots \ldots \ldots \ldots, 10$

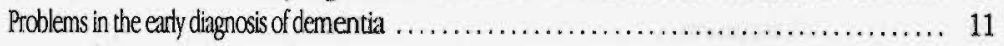

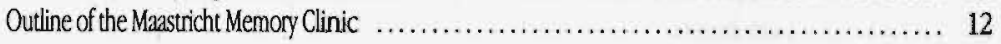

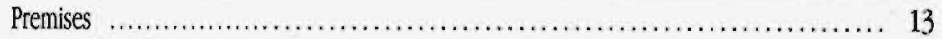

Outine of the diagnostic procedure $\ldots \ldots \ldots \ldots \ldots \ldots \ldots \ldots \ldots \ldots \ldots \ldots \ldots \ldots \ldots \ldots, 13$

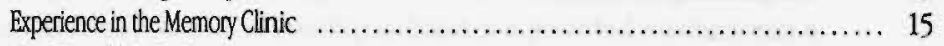

Aims and outline of this thesis ...........................................

II The assessment of mild dementia: diagnostic concepts and methodological

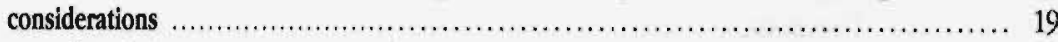

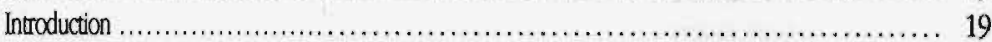

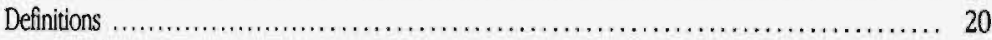

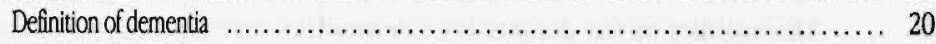

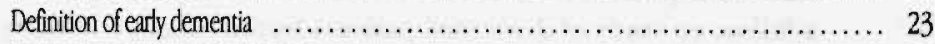

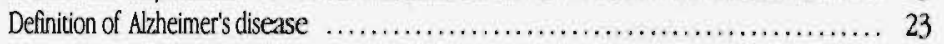

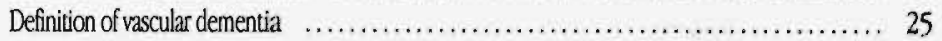

Phenomenology of the prodromes of dementia ................................ 26

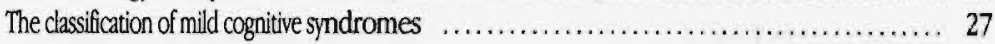

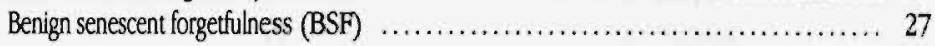

Age-associated memory impairment $\ldots \ldots \ldots \ldots \ldots \ldots \ldots \ldots \ldots \ldots \ldots \ldots \ldots, 28$

DSM-III-(R) criteria: mild dementia . .................................... 29

Global deterioration scale: very mild and mild cognitive decline $\ldots \ldots \ldots \ldots \ldots \ldots . . \ldots$

Clinical dementia rating scale: questionable and mild dementia $\ldots \ldots \ldots \ldots \ldots \ldots . \ldots . \ldots . \ldots$

CAMDEX: minimal dementia and mild dementia $\ldots \ldots \ldots \ldots \ldots \ldots \ldots \ldots \ldots \ldots, 31$

Comparison of the different criteria for mild cognitive syndromes $\ldots \ldots \ldots \ldots \ldots \ldots \ldots \ldots 32$

Clinical and methodological issues .......................................... 34

The continuum between normal ageing and dementia ........................ 34

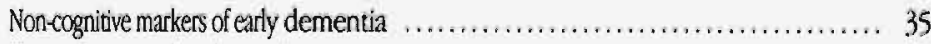

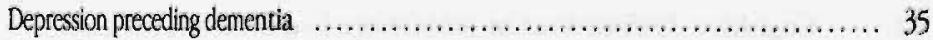

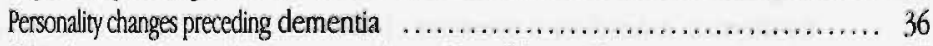

Subjective cognitive complaints as an early marker of dementia .................. 37

Who does the assessing? ............................................ 38

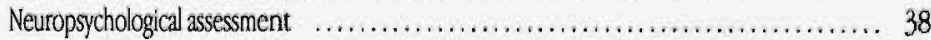

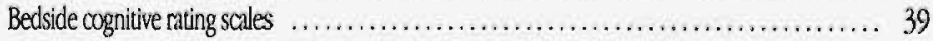

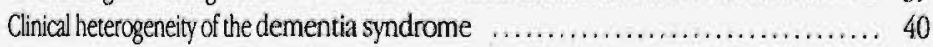

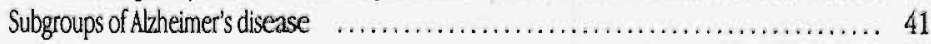

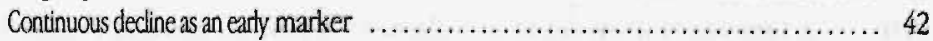

Age and comoridity $\ldots \ldots \ldots \ldots \ldots \ldots \ldots \ldots \ldots \ldots \ldots \ldots \ldots \ldots \ldots \ldots, 42$

Condusions ........................................................ 42 
III Diagnosing dementia:

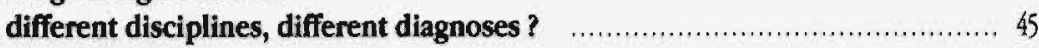

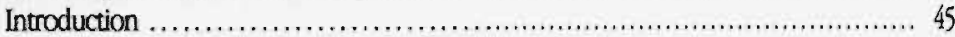

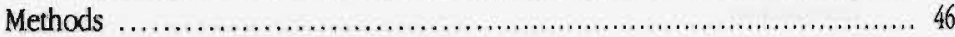

Results .................................................................... 49

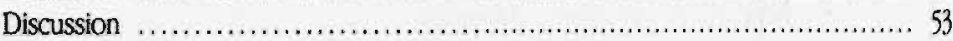

Condusion ......................................................................... 55

IV Diagnosing dementia:

a comparison between a monodisciplinary and a multidisciplinary approach ........ 57

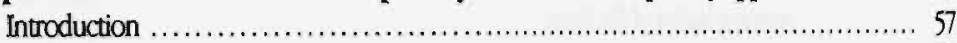

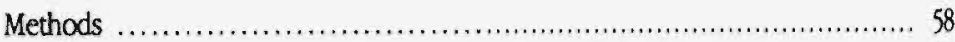

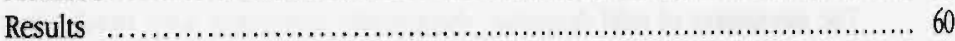

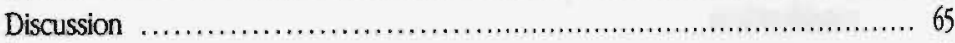

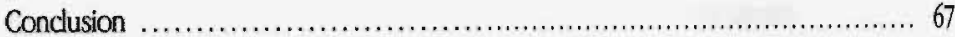

V The validity of the diagnosis of dementia:

a follow up study of demented patients from the Maastricht Memory Clinic .......... 69

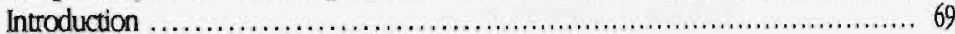

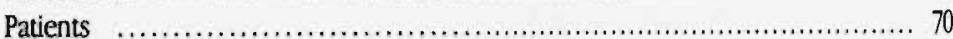

Methods …......................................................................... 70

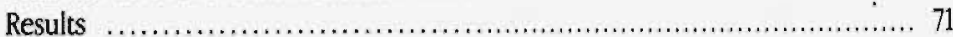

Discussion ........................................................................ 73

VI Psychiatric disorders in patients attending an outpatient memory clinic ............. 75

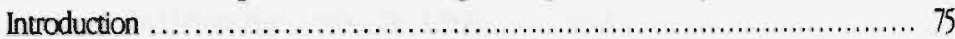

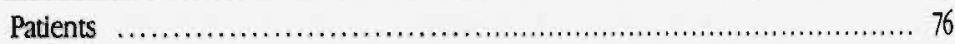

Methods ................................................................... 7

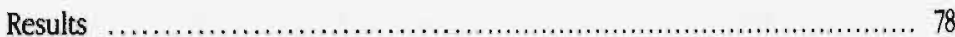

Discussion ................................................................. 81

Condusion ................................................................. 82

VII Cognitive dysfunctions in middle-aged subjects with late-onset dysthymia attending a memory clinic ............................................. 83

Introduction ............................................................ 85

Materials and methods ........................................................ 87

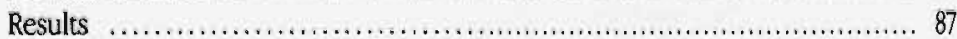

Discussion ................................................................. 89

Condusion .............................................................. 92

VIII A two-year follow-up of non-demented patients attending a memory clinic .......... 93

Introduction .................................................................. 93

Patients and methods ............................................................

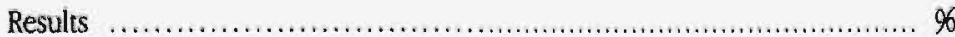

Discussion ..................................................................... 101 


\section{TABLE OF CONTENT}

IX A comparison between six current sets of criteria

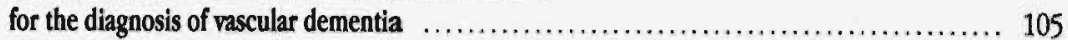

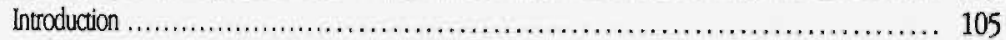

Patients .............................................................. 106

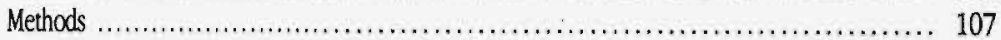

Results ......................................................... 107

Discussion ...................................................... 111

X Depression, insight and personality changes in Alzheimer's disease and vascular

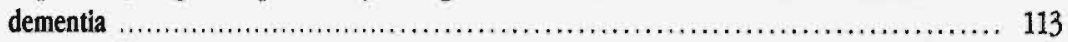

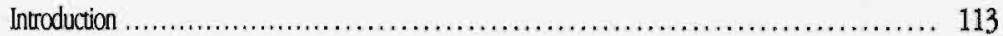

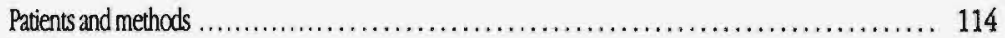

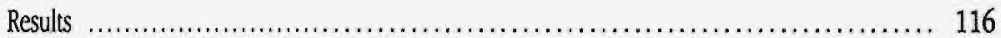

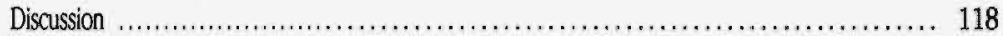

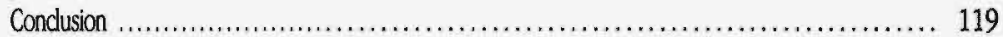

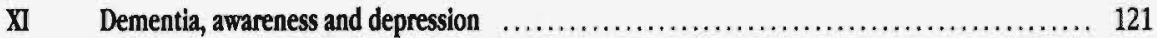

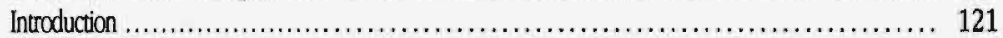

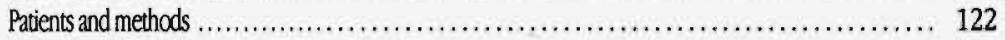

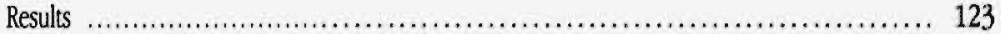

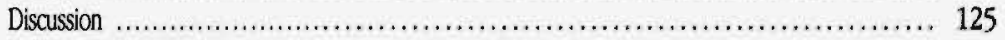

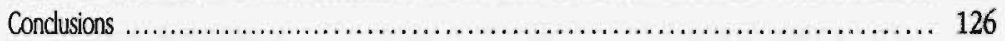

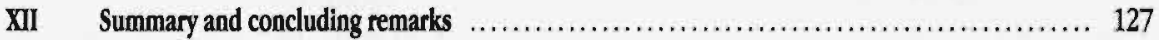

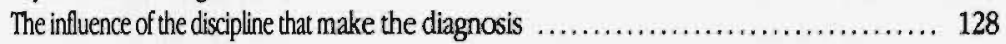

Clinical aspects of non-demented patients with cognitive complaints ................. 130

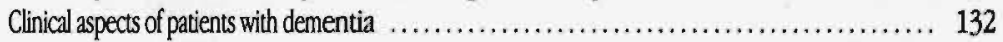

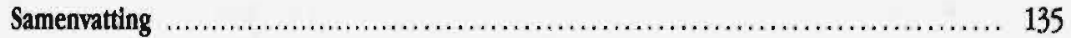

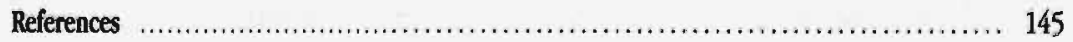

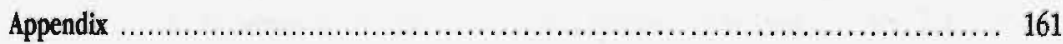

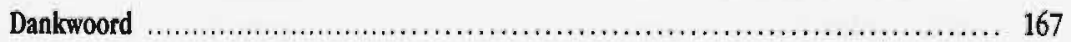

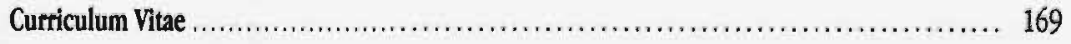

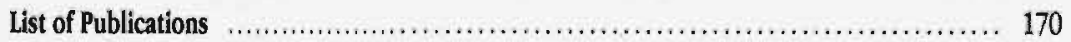




\section{Introduction}

Of the many disabilities associated with old age, dementia (to lose one's' mind, in Latin) is perhaps the most appalling. Approximately 1.2 million people in the United States [156] and 100,000 people in the Netherlands [306] suffer from severe dementia. Estimates for mild dementia are twice as high. These numbers are on the increase in view of the anticipated 'old-age-boom'. In the last decade, dementia has been increasingly recognized as a major public health problem, not least because the psychological and physical burden on caregivers is enormous. Moreover, dementia has an immense effect on society in terms of consumption of medical facilities [161]: the costs of dementia in terms of nursing homes and other forms of care in the United States are calculated at about $\$ 40$ billion per year [225].

The accurate diagnosis of dementia has long been a problem, but in the last decade considerable progress has been made. Consensus procedures have yielded operational criteria for the clinical diagnosis of dementia and the main dementing illnesses [7, 45, 207, 266]. Essentially, the diagnosis of dementia involves two steps: first, the diagnosis of the behavioural syndrome of dementia, and second, the differentiation of diseases that produce dementia. According to current definitions, dementia is a condition of functional dependency [7]. The syndrome of dementia should be differentiated from normal ageing and psychiatric disorders such as depression, delirium and focal neuropsychological deficits. Although dementia can be caused by more than 70 disorders [115], Alzheimer's disease (AD) and vascular dementia (VD) account for about $80 \%$ of all demented patients [156]. The main problem is the diagnosis of $\mathrm{AD}$, which is still largely arrived at by excluding other dementing disorders, with dementia and a progressive course being the only two inclusion criteria. It is evident that an insidious and progressive disease such as $\mathrm{AD}$ does not manifest itself from the very beginning as the complete syndrome of dementia. Early in the course of $\mathrm{AD}$, the patient may exhibit mild 
memory impairments and changes in other mental domains that are not sufficiently severe to merit the diagnosis of dementia. Recognizing these prodromes of $\mathrm{AD}$ is one of the most complex problems in psychogeriatry to date.

The early and differential diagnosis of dementia is the main topic of this thesis. Before discussing the clinical studies that have been performed on this subject, we will discuss shortly the rationale of an early diagnosis of dementia. Most of the studies described in this thesis were carried out in the Memory Clinic of the University Hospital of Maastricht, the Netherlands. The organization of this clinic will also be described briefly. This chapter will also provide a description of the major questions that this thesis attempted to answer.

\section{Rationale of the need for an early diagnosis of dementia}

At present, an accurate diagnosis of early dementia has become a major objective of scientific research and clinical practice $[56,116,124,152,162,219]$. There are many reasons for this.

From a scientific point of view, the diagnosis of early $\mathrm{AD}$ helps to clarify epidemiological aspects such as the natural history of the disease, which is of major importance for planning and developing health-care facilities. The lack of clear diagnostic criteria for early $\mathrm{AD}$ is reflected by the estimates of its prevalence. In contrast to severe dementia, in which the prevalence is remarkably consistent in various studies (about $5 \%$ of the population of 65 years and older), the prevalence of mild dementia ranges from 2.6 [19] to $21.9 \%$ and even up to $52.7 \%$ in one Japanese study [124]. This extreme variation is probably mainly due to differences in the criteria and the methods used to identify cases of mild dementia $[123,124]$. Second, studying the phenomenology of the very first manifestations of $\mathrm{AD}$ provides information about the specific brain regions involved, and this helps to clarify the underlying cause. For instance, early onset Alzheimer's disease presents relatively often with language disorders, suggesting that the left hemisphere may be more vulnerable to the early-onset variant, compared with the late-onset type. Observations such as this have led to the notion of heterogeneity of Alzheimer's disease [27, 200]. Third, it is of vital importance that drugs that enhance cognition in demented patients are tested in the early phases of the disease, before changes in the brain have become structural $[151,336]$.

From a clinical point of view, an early diagnosis of $A D$ is also of importance. First, a significant minority of patients with the diagnosis of dementia suffer from treatable conditions $[104,116,197,290]$, and treatment of these diseases is expected to be more successful in the early stages $[47,180]$. Additionally, the focus of geriatric medicine has shifted during the last decade from the cure of disease to the reduction of 'excessive disability' [13]. This approach supports the idea of an early elective methodological examination in search of treatable concurrent conditions, such as drug intoxication, paranoia or sensory impairment, even when the patient is known to be suffering from irreversible dementia. Second, remedial psychological treatments designed to compensate for the cognitive deterioration of irreversible dementia are likely to be more effective in mild than in severe dementia [101, $129,210,341]$. Third, a diagnosis of mild dementia is important with regard to the future. It 
enables the health caregivers to plan measures to avoid crises and relieve some of the distress of the patients' relatives. The caregivers are thus able to anticipate future measures such as placement of their relative in a nursing home, and they can commence a process of acceptance and realistic coping, thus preventing possible misunderstandings and counterproductive interactions $[110,230,339]$. Fourth, an early diagnosis carries implications for the physician's management of the patient and his or her relatives. Knowledge of the existence of a dementia syndrome has, for example, an influence on the choice of drugs prescribed, notably those with anticholinergic properties $[56,124]$. In addition, early recognition of dementia has an impact on the interpretation of information from the patient's history and should lead to a heightened awareness on the part of the physician. Fifth, it is of great importance to have diagnostic methods that accurately sort out those patients with mild dementia from those with more benign forgetfulness. This in the light of the public's growing awareness of dementia as a major health problem and the corresponding anxiety that is caused by the media $[147,241$, 318].

Active detection of people with cognitive deterioration has also raised some scepticism about the ethical aspects of the consequences for the individual patient, who is left with a dramatic diagnosis for which no cure exists [56]. However, although this criticism may apply to community studies, where people are faced with this diagnosis without having asked for this, it does not apply to daily clinical practice, where an increasing number of elderly people complain about their memory and seek help for their cognitive problems [51, 191]. Memory impairment may compromise the individual's ability to function in intellectually demanding activities and employment situations. Subjects with memory complaints may experience great distress and are not comforted by assurances that the impairment is normal, that they are "just getting older", or that they are "not becoming demented" [59].

\section{Problems in the early diagnosis of dementia}

Many efforts have been made to find objective biological markers for an early diagnosis of Alzheimer's disease; however, none of these markers has as yet proven to be successful in individual cases. Clinical neurological findings for mildly demented patients did not differ from those of normal subjects $[107,213]$. A considerable overlap between normal subjects and mildly demented patients exists in the findings obtained by Computed Tomography (CT) [69, 144], Single Photon Emission Computed Tomography (SPECT) [331] and Magnetic Resonance Imaging (MRI) [280]. Studies with Positron Emission Tomography (PET) have shown more encouraging results [122, 249], but PET is not practical because of its enormous costs and its limited availability. Progress has been made in the development of biochemical markers by using cerebro-spinal fuid from patients with $\mathrm{AD}$, but this still in an experimental phase [109, $112,287]$. Thus, at present biological markers may be of value in differentiating Alzheimer's disease from other dementing illnesses [144] , but not in accurately predicting dementia in subjects who are not yet demented. Neuropathological data from patients in the early stages of $\mathrm{AD}$ are extremely rare and not representative $[60,126,216]$. Given the lack of reliable and 
valid aetiologically based objective tests, the diagnosis of early Alzheimer's disease is still based on detailed clinical description, differentiation from normality and other behavioural diagnoses such as depression, neuropsychological examination and follow-up studies [227, 264].

Of these aspects, the differentiation between normal states and dementia has been especially problematic. In the past 30 years, several attempts have been made to define the clinical and neuropsychological features of the borderline state between normality and frank dementia. Already in $1958 \mathrm{Kral}$ introduced the terms benign and malignant senescent forgetfulness to describe the memory complaints of the elderly he studied [170]. Since then, investigators have tried to describe these states in more detail, eg, very mild or mild cognitive decline [255], questionable dementia [139], minimal dementia [272], age-associated memory impairments [59], age-consistent memory impairment and late-life forgetfulness [23]. The fourth edition of the Diagnostic and Statistical Manual of Mental Disorders (DSM-IV) encompasses a new category as well, namely 'age-associated cognitive decline' [37, 248, 292]. However, the nosological status of many of these proposals is still unclear, especially with regard to what extent they are related to dementia [68]. Despite enormous scientific effort, there are still many uncertainties about the diagnosis of the very first stages of Alzheimer's disease, i.e., before it is manifest as frank dementia. These difficulties in diagnosis are largely inherent to the multidimensional nature of the condition.

It is a common experience of those who are active in the field of dementia that patients only come to the notice of health professionals once they are already clearly demented. This may be because up till now there were only a very few facilities that were adequately equipped for the diagnosis and treatment of the less disturbed patient. As a consequence, traditional psychogeriatric departments 'screen' patients who have already been dementing for some time for potentially treatable causes. In recent years, special memory clinics have been set up in several places for the evaluation and management of elderly patients with milder cognitive deficits $[4,14,38,241,319,328]$. In contrast to traditional psychogeriatric departments, the criterion for referral to memory clinics is usually a mild or moderate cognitive problem, and not severe dementia. The threshold for referral is therefore lower, resulting in earlier and better opportunities for preventive interventions. The diagnostic approach in a memory clinic differs on important aspects from that of the more traditional psychogeriatric departments. For example, the first question is not what causes the dementia, but rather whether dementia is present or not. The specific goals of the memory clinics make specific demands upon the organization and on the diagnostic procedures used. Existing memory clinics differ among themselves in the diagnostic approach and their specific goals [150]. The aims, methods and organization of the Maastricht Memory Clinic (MMC) of the University Hospital of Maastricht, in which most of the studies of this thesis were carried out, is described here. 


\section{Outline of the Maastricht Memory Clinic}

The MMC opened in May 1986. It was a joint effort of the Department of Neuropsychology and Psychobiology, University of Limburg, and the Departments of Psychiatry and Neurology of the University Hospital of Maastricht, the Netherlands $[319,322]$. The explicit aims of the MMC are to provide a new service for the diagnosis and treatment of patients with relatively mild memory problems, whether demented or not, and to perform clinical research with particular reference to the very early stages of Alzheimer's disease. The following sections describe the main aspects with regard to the premises, organization and diagnostic procedures of the MMC. More details of the procedures used are provided in chapters 5, 6, and 7.

\section{Premises}

Before the MMC was opened, some principles were formulated that formed the basis of the working methods to be used. These can be summarized as follows. First, the diagnostic procedures should be based upon clear and well-accepted diagnostic research criteria so that the clinic can be used for research goals as well. Second, a variety of cognitive and noncognitive behavioural aspects should be assessed, because it is not know which features are particularly distinctive for the diagnosis of early dementia. Third, the diagnostic approach should be multidisciplinary, with equal input from psychiatry, neurology and neuropsychology. It was felt that each specialty complements the other with its own specific paradigms, specialistic expertise and investigational methods. Fourth, in order to enable group comparisons, the diagnostic procedure must be standardized as much as possible, by using reliable quantitative measures of the different aspects involved. Fifth, the instruments used must be sensitive enough to assess subtle impairments and changes with time, and must have also a difficulty range appropriate for non-demented elderly and for early Alzheimer's disease in order to avoid floor and ceiling effects. Ideally, both non-demented and demented subjects should be evaluated with the same instruments, in order to allow for follow-up studies. Sixth, the tests should be documented in terms of reliability and validity, and norms should be available as well as equivalent forms for repeated administration. Finally, and most importantly, the clinic should not only be a centre for diagnosis, but must also offer specific treatment and care, in order to meet the expectations of the patients. Such a department should work closely with other disciplines involved in the care of the demented patient.

\section{Outline of the diagnostic procedure}

In the MMC, the criteria of the DSM-III ( and from 1987, the DSM-III-R) are used for the diagnosis of dementia and other psychiatric syndromes. The criteria of the National Institute of Neurological and Communicative Disorders and Stroke and the Alzheimer's Disease and Related Disorders Association (NINCDS-ADRDA) are used for the diagnoses of possible and probable Alzheimer's disease [207]. Hachinski's Ischaemic Score is used for the diagnosis of 
vascular dementia. Other neurological and somatic conditions are diagnosed according to usual clinical guidelines. All patients are examined by, or under the direct supervision of, the author of this thesis, who is specialized in both psychiatry and neurology, and experienced in the area of overlap between the two disciplines. In addition, many patients are examined by a neurologist who has a special interest in neurodegenerative brain diseases. The neuropsychiatric part of the assessment lasts about 1.5 to 2 hours, and information is recorded in a specially designed standardized patient file. The following scales are routinely administered, in order to document several important behavioural aspects: the Global Deterioration Scale, to obtain a measure of the extent of global deterioration [255]; the Blessed dementia scale, as an overall measure of social functioning [24]; Folstein's' Mini Mental State Examination [97] in order to standardize the clinical impression of cognitive functioning; and Hamilton's depression rating scale, as a symptom checklist for affective symptomatology and in order to obtain an overall measure of depression [120]. A CT scan and ancillary laboratory tests, according to general consensus [281], are performed when there are objective cognitive deficits.

The neuropsychological part of the assessment lasts another 2 to 2.5 hours and has been described in length elsewhere [146]. Briefly, this part involves standard psychometric tasks, combined with a neurobehavioural examination according to Luria-Christensen $[42,195]$ and information processing tasks [297], to assess the relevant cognitive functions in the areas of memory, attention, language, praxis, perceptual functions, speed of information processing and other aspects. The battery of neuropsychological tests has been designed in order to contribute to the classification of memory disorders (e.g., dementia or age-associated memory impairments), but also to provide insight into the psychological mechanisms that lead to a certain performance level. Special emphasis is also placed on the use of qualitative tests, so that profiles can be made of the weak and strong aspects of cognitive functioning, thereby making it possible to offer better opportunities for specific advice and guidance as compared when quantitative tasks are used.

After the tests have been completed, the results are discussed at a weekly interdisciplinary meeting. After discussion in this forum, a definite syndromal and aetiological (differential) diagnosis is made, and treatment proposals are formulated. Finally, a letter is sent to the referring physician in which all the diagnostic elements, a final diagnosis and the treatment proposal are described. Once the assessment phase is completed, the diagnostic data are entered into a database.

The MMC cooperates with other regional departments involved in the care of patients with dementia, eg, geriatricians and the psychogeriatric department of the Regional Institute of Ambulatory Mental Health Care (RIAGG). The MMC also participates in the psychogeriatric platform, a weekly meeting at which requests for nursing home admissions are discussed. In addition to psychiatric and neurological treatments, patients of the MMC are offered the chance to participate in ongoing clinical trials with experimental cognition-enhancing drugs and putative anti-Alzheimer agents. Moreover, a psychological treatment programme has been developed with the aim of improving coping and compensation strategies with regard to the cognitive deficits $[50,260]$. 


\section{Experience in the Memory Clinic}

Based on our experience in the MMC, we agree with others $[4,14,38,52,241,328]$ that memory clinics offer an excellent opportunity for combining specific patient care and clinical research into memory impairments and early Alzheimer's disease. At present (May 1993), more than 600 patients have been examined in the MMC. The mean age of the first 430 patients was 61.7 years, ranging from 16 to 88 years. Thirty-five per cent fulfilled the DSM-III-R criteria of dementia, and cognitive impairments could be objectified in another $49 \%$. Sixteen per cent of the patients appeared to have normal cognitive functions on extensive neuropsychological testing. These figures differ from those from traditional geriatric departments and clearly reflect the idea that the MMC is a memory clinic, not a dementia clinic [150].

Some particular problems and limitations of this type of department should be mentioned as well. The diagnostic work-up is time consuming and requires experience, because it encompasses several elements for which clinical judgement is needed. The balance between costs and benefit from the point of view of patient care has still to be examined, although memory clinics definitely contribute immaterially by removing uncertainties and by offering help to patients and their caregivers in dealing with their cognitive deficits. As matters regarding memory having become fashionable, one takes the risk that an expensive service is available for the 'worried well' who merely want to train their unimpaired memory. This is avoided by ensuring that all patients are referred by their physicians before they are assessed in the MMC. However, an intense fear of dementia, whether justified or not, may be a good reason for further evaluation of the subject in the MMC. Finally, the multidisciplinary type of assessment makes special demands on the organization, as the assessments of each specialty have to be combined into an integrated final diagnosis and treatment plan. This means that extra motivation and enthusiasm on the part of those involved are essential to the success of the organization.

\section{Aims and outline of this thesis}

This thesis addresses clinical aspects of an early diagnosis of dementia, with particular reference being paid to the differentiation between early dementia and normal age-associated cognitive impairments and depression. The results of nine different clinical studies are presented in the next chapters. The main subjects of these studies deal with the differences between the specialties involved in the diagnosis of dementia, the clinical characteristics of patients who seek help for cognitive complaints, and the use of clinical features in the differential diagnosis of dementing disorders. The relevant literature is reviewed first (chapter 2), in order to depict the current state of the subject. 
The main questions of this thesis are:

1 What are the differences between the diagnostic outcome of the various specialties involved in the assessment of dementia?

As will be shown in the comprehensive review of the literature (chapter 2), the diagnosis of early Alzheimer's disease is dependent to an important extent on clinical judgement. As a consequence, theoretically important differences may be expected between the various disciplines involved, but there are hardly any data on this subject. A related question is whether a multidisciplinary approach, as is generally recommended, leads to substantial differences in the diagnostic outcome of patients with memory impairments compared to the outcomes achieved with a monodisciplinary type of assessment, as is usually the case in daily clinical practice. Therefore, two studies were carried out. The first study, described in chapter 3, was an experimental study of the interdisciplinary agreement on the diagnosis of dementia among the participants of a consensus meeting on dementia, which was held in Utrecht, the Netherlands, in November 1988. Representatives of several different specialties were asked to diagnose ten fictitious patients with some kind of memory problem. In chapter 4, a study is presented in which the monodisciplinary approach of referring physicians was compared with the multidisciplinary diagnostic approach of the MMC. In order to investigate the validity of the diagnoses of dementia that were made by the MMC, a follow-up study involving patients diagnosed as demented was carried out into the course of social and cognitive functioning. The results of this study are presented in chapter 5 .

2 What are the clinical characteristics of non-demented patients who seek belp for cognitive impairments

On the basis of the literature, it can be concluded that it is important to examine nondemented patients with cognitive complaints and various psychiatric disorders in more detail, as they may provide important clues about the very first manifestations of Alzheimer's disease. Therefore, three studies were carried out with patients from the MMC who had cognitive impairments milder than those meriting the diagnosis of dementia, in order to answer the following questions:

2.1 What psychiatric disorders, apart from dementia, can be accompanied by cognitive complaints or cognitive disorders?

2.2 Can neuropsychological dysfunctions be objectified in non-demented patients with relatively mild complaints about mood, memory and concentration?

2.3 How do cognitive complaints and lowered mood relate to each other. And bow do these neuropsycbological deficits develop furtber?

The study described in chapter 6 provides an inventory of the various psychiatric disorders that may be related to cognitive complaints. Chapter 7 describes a study in which 25 patients with 
a dysthymic disorder were compared to 25 normal subjects of the same age, sex and educational level, in order to investigate whether cognitive deficits could be objectified. A 2-year follow-up study of non-demented patients from the memory clinic is presented in chapter 8. This study was carried out in order to obtain insight into the development of the cognitive impairments, and their relationship with depressive symptomatology.

\section{Are bebavioural characteristics of use in establishing the aetiology of dementia?}

Alzheimer's disease remains a diagnosis that is largely made by excluding other disorders. Thus, the reliability of this diagnosis is directly related to the accuracy with which other disorders can be diagnosed or ruled out. The most frequent disorder that should be differentiated from Alzheimer's disease is vascular dementia. For a long time, this diagnosis was made on the basis of clinical features, with ischaemic scores generally being used as the most important diagnostic tools. The introduction of new diagnostic criteria for vascular dementia puts more emphasis on the results of data collection from brain-imaging techniques such as CT or MRI scan. This led us to pose the following questions:

\subsection{What are the differences between current criteria for vascular dementia and bow great is the impact on the diagnosis of Alzbeimer's disease? \\ 3.2 Can the differentiation between Alzheimer's disease and vascular dementia be made reliably on the basis of behavioural cbaracteristics?}

In Chapter 9, several current diagnostic criteria for the diagnosis of vascular dementia are compared with each other in patients from the MMC in whom disorders other than cerebral ischaemia and primary degenerative brain disease have been excluded. In chapter 10 , the results of a study of some behavioural characteristics that are often presumed to differentiate between Alzheimer's disease and vascular dementia, such as depression and retained insight, are presented.

\subsection{Is the degree of awareness of being cognitively impaired related to the depression in patient with dementia?}

Depression is an important disorder that can precede, mimic, coexist with or result from dementia. The prevalence of depression, or at least depressive symptoms, is reported to be generally higher in patients with dementia than in those without. The pathogenesis of these depressive symptoms is not well understood and can be explained both biologically and psychologically. In chapter 11 the relationship between awareness and depression in demented patients is investigated, in order to determine whether patients who know that they are deteriorating are more at risk of developing depression than those who lack such knowledge.

The main findings and the possible implications of the above-mentioned studies are discussed in chapter 12 . 



\section{The assessment of mild dementia: diagnostic concepts and methodological considerations*}

\section{Introduction}

The clinical and scientific importance of an early diagnosis of dementia was described in the previous chapter of this thesis. In this chapter, we address some relevant issues with regard to current concepts and definitions of dementia, with particular reference to Alzheimer's disease, which is the most frequent cause of dementia. Various diagnostic criteria of the states between normality and frank dementia are discussed. In particular, we pay attention to the validity of these criteria in terms of prognostic significance. The purpose of this review is to identify problem areas that influence the progress of research into the diagnosis of mild dementia. Another purpose of this review is to distil from the literature the clinical phenomenology of the prodromes of dementia, and to identify specific features that may predict progressive mental decline in the very early stages of $\mathrm{AD}$.

*Parts of this chapter have been published in: Verhey FR], Jolles, J. Over de spraakverwaming rond het begrip dementic en de ziekte van Alzheimer. Tijdschrift v Gerontologie en Geriatrie 1988; 19: 89.96. 


\section{Definitions}

Historical reviews of the concept of dementia have been presented elsewhere $[21,74,186]$. Daily practice shows that the way terms are used varies considerably. Therefore, it is essential to consider the various definitions of dementia and of the two most frequent dementing disorders, Alzheimer's disease and vascular dementia.

\section{Definition of dementia}

The term dementia was probably used for the first time in the 17th century [21]. Dementia is now generally regarded as a clinical syndrome and not as a disease, but important differences exist in the interpretation of this notion. To quote Wells: "the word has successfully defied attempts to limit its meaning and to fix its definition" [333]. This leads to semantic confusion, which has a negative influence on health care and on the progress of scientific research. Various meanings of the word dementia are summarized in Table 1.

The term dementia is used by some researchers to denote a clinical condition, defined only by observable behaviour, irrespective of its aetiology. For instance, the report on the NINCDS/ADRDA criteria for clinical Alzheimer's disease states: "Dementia is a diagnosis based on behaviour and cannot be determined by computerized tomography, electroencephalography, or other laboratory instruments, although specific causes of dementia may be identified by these means" [207]. The consequence of this definition is that dementia can be caused by both organic and functional disorders. Others limit the definition of dementia to organic aetiologies. For instance, the DSM-III-R states: "Either there is evidence (...) of specific organic factor(s) judged to be aetiologically related to the disturbance, or, in the absence of such evidence, an aetiologic factor can be presumed if the disturbance cannot be accounted for by any non-organic mental disorder, e.g., major depression accounting for cognitive impairment" [7]. Still others have extended the definition of dementia by the characteristics of irreversibility or a progressive course [272]. In common clinical practice and for lay people, this use of the term is perhaps the most frequent, although it is not supported by any official source. Finally, the term is also used as an equivalent for primary degenerative diseases, and is probably meant to imply Alzheimer's disease [294]: a progressive mental decline caused by a particular irreversible brain disorder.

One is not always fully aware of the fact that the term dementia is a construct only defined by consensus agreement. Confusion arises when different meanings are used without further explanation. Thus, a diagnosis of dementia means to one clinician that a thorough examination should be carried out, whereas the other might perceive patients with the same diagnosis as untreatable. In order to avoid this counterproductive confusion, it is important that all elements of the diagnosis -aetiology, prognosis and clinical phenomenology- are named separately [315]. 
Table 1: Different meanings of the word dementia

\begin{tabular}{|c|c|c|}
\hline Definition of dementia & Author, year & Reference \\
\hline \multirow{4}{*}{$\begin{array}{l}1 \text { Clinical syndrome without any } \\
\text { reference to aetiology }\end{array}$} & NINCDS/ADRDA 1984 & {$[207]$} \\
\hline & Small 1982, 1983 & {$[288,289]$} \\
\hline & Verhey, 1988 & {$[315]$} \\
\hline & Schulte, 1989 & {$[40,281]$} \\
\hline 2 Clinical syndrome with & Kuiper, 1973 & {$[174]$} \\
\hline $\begin{array}{l}\text { progressive course and } \\
\text { imeversibility, without any } \\
\text { reference to aetiology }\end{array}$ & Foster, 1990 & {$[100]$} \\
\hline \multirow{7}{*}{$\begin{array}{l}3 \text { Clinical syndrome with organic } \\
\text { aetiology }\end{array}$} & Wells, 1977 & [333] \\
\hline & DSM-III, 1980, 1987 & {$[6,7][63]$} \\
\hline & Cummings, 1983 & {$[103][198][58][54]$} \\
\hline & Prederiks, 1985 & \\
\hline & Marsden, 1985 & \\
\hline & van Crevel, 1986 & \\
\hline & NIH, 1987 & \\
\hline $\begin{array}{l}4 \text { Clinical syndrome with organic } \\
\text { aetiology and progressive course }\end{array}$ & $\begin{array}{l}\text { Godderis, } 1985 \\
\text { Roth, } 1986\end{array}$ & [111] [272] \\
\hline $\begin{array}{l}5 \text { Clinical syndrome with organic } \\
\text { aetiology,progressive course and } \\
\text { imeversibility }\end{array}$ & & \\
\hline $\begin{array}{l}6 \text { Clinical syndrome with organic } \\
\text { aetiology,progressive course, } \\
\text { irreversibility and specific } \\
\text { neuropathological features }\end{array}$ & Stam, 1985 & [294] \\
\hline
\end{tabular}

The most widely used definition of dementia to date is that of the DSM-III-R [7] (see Table 2). In short, the DSM definition involves a combination of memory impairment with at least one of the domains of abstract thinking, judgement, aphasia, apraxia or agnosia, constructional difficulty, or personality changes. The impairment must be severe enough to interfere with social functioning. It is important to note here that the DSM-III-(R) definition implies the acceptance of some degree of heterogeneity: not all cognitive domains are necessarily affected.

There are some problems with the DSM-III-R definition. First, the inclusion of an organic aetiology raises logical problems. Because functional dementia (often referred to as pseudodementia) may present with clinical picture simular to that of organic dementia $[78,288$, 289], the distinction between the two is made on the basis of the presumption that there is an organic cause underlying dementia. However, to make assumptions in order to fulfil diagnostic 
criteria is logically untenable $[196,315]$. Second, the DSM criteria lead to a diagnostic dilemma, because they require the exclusion of functional disorders such as depression, but the criteria for depression require the exclusion of organic disorders.

Table 2: DSM-III-R criteria for dementia [7]

1 Demonstrable evidence of impairment in short- and long-term memory. Impairment in short-term memory (inability to learn new information) may be indicated by an inability to remember three objects after five minutes. Long-term memory impairment (inability to remember information that was known in the past) may be indicated by an inability to remember past personal information (e.g., what happened yesterday, birthday, occupation) or facts of common knowledge (e.g., past Presidents, well-known dates).

2 At least one of the following:

1 impairment of abstract thinking, as indicated by an inability to find similarities and differences between related words, difficulty in defining words and concepts, and similar tasks

2 impaired judgement, as indicated by an inability to make reasonable plans to deal with interpersonal, family and job-related problems and issues

3 other disturbances of higher cortical function, such as aphasia (disorder of language), apraxia (inability to carry out motor activities despite intact comprehension and motor function), agnosia (failure to recognize or identify objects despite intact sensory function), and "constructional difficulty" (e.g., inability to copy three-dimensional figures, to assemble blocks, or to arrange sticks in specilic designs)

4 personality change, i.e. alteration or accentuation of premorbid traits

3 The disturbances 1 and 2 significantly interfere with work or usual social activities or relationships with others.

4 Not occurring exclusively during the course of delinum

5 Either (1) or (2):

(1) there is evidence from the history, physical examination, or laboratory tests of a specific organic factor or factors) judged to be aetiologically related to the disturbance

(2) in the absence of such evidence, an aetiologic organic factor an be presumed if the disturbance cannot be accounted for by any non organic mental disorder, e.g. Major Depression accounting for cognitive impaiment

DSM III-R criteria for severity of dementia:

Mild : Although work or social activities are significantly impaired, the capacity for independent living remains, with adequate personal hygiene and relatively intact judgement.

Moderate : Hazardous independent living; some degree of supervision is necessary

severe: Activities of daily living are so impaired that continual supervision is required, e.g, unable to maintain personal hygiene; largely incoherent or mute.

Third, the evidence of memory impairment is demonstrated by difficulties in remembering recent or past information. However, the inability of a patient to name this information does not automatically mean that the information is lost. Therefore, the criteria may be more difficult to apply in patients with subcortical dementia, in whom recall is impaired but 
recognition is relatively intact $[3,71,137]$. The distinction of several subgroups of dementia will be discussed in detail in another section of this chapter. Fourth, the criteria do not define the minimal duration of the symptoms, nor do they require a normal premorbid level, although most clinicians will agree that these aspects should be included in the definition. Fifth, the DSM-III criteria have been critized for treating the underlying dimensional concept of dementia as too categorical [152].

Despite these limitations of the DSM-III(-R) criteria, virtually all current research studies make use of them. It should be born in mind that the DSM-III-(R) is essentially a researchorientated instrument. It was not designed to give diagnostic refinement. The main goal is to reduce the number of false-positive diagnoses, in order to obtain homogeneous samples and to keep any distortion or dilution of statistical data at the lowest possible level [154]. Therefore, the threshold for inclusion has been set rather high. In contrast, clinical practice must insist on a minimum of false-negative diagnoses. The differences in these two starting points should be noted, especially when applying the criteria for mild dementia. The use of these criteria has had a major impact on clinical research, and diagnostic accuracy has improved significantly since the introduction of these criteria. In this thesis we make use of the DSM-III-R criteria of dementia, except for the last criterion concerning the presumed organic aetiology. The organicity criterion was also abandoned at the Dutch consensus meeting on the diagnosis of dementia, in Utrecht, November 1988 [40].

\section{Definition of early dementia}

The issue of early dementia is dealt with in the literature in at least two different ways. First, the term refers to the detection of cases of mild or moderate dementia in order to prevent crises and to be able to take the necessary action electively, rather than ad hoc in a crisis situation (e.g., [276]). In this context, it is more correct to speak of the detection of mild, instead of early, dementia, since the latter unjustly suggests a progressive course as part of the definition of dementia.

Second, the issue of early dementia applies to the detection of the prodromes of dementing disorders, i.e., before the disease has developed into dementia (e.g., [1]). According to current definitions, a diagnosis of Alzheimer's disease implies the clinical syndrome of dementia [7, 207]. A semantic problem should be noted here, as this definition implies that Alzheimer's disease in its predementing stages is a contradiction in terms, which is illogical. It is better to define this second approach as the detection of early Alzheimer's disease rather than as the detection of early dementia.

\section{Definition of Alzbeimer's disease}

In 1907, Alois Alzheimer [5] described a patient with progressive dementia, caused by a disease that Kraepelin three years later named 'Alzheimer's disease' [127, 169]. Alzheimer's disease is characterized pathologically by cerebral deposits of abnormal fibres in senile plaques and neurofibrillar tangles. The term Alzheimer's disease has been used in different contexts: to 
denote an onset of dementia before the age of 65 years (as opposed to 'senile dementia', after this age) [159]; for patients who show the clinical triad of aphasia, apraxia, and agnosia (as opposed to 'dementia simplex', without this triad) [55]; and as a neuropathological diagnosis (as opposed to presenile or senile dementia of the Alzheimer Type, in the absence of pathological verification) $[16,187]$. The term 'disease' unjustly suggests a distinct nosological entity, because there is not one single cause of Alzheimer's disease. Case-control studies have shown various risk factors to be associated with Alzheimer's disease, of which age and a family history of dementia are the most prominent [75].

At present, two sets of criteria for $\mathrm{AD}$ are widely used: the DSM-III-R criteria for Primary Degenerative Dementia of the Alzheimer Type (PDD) [7] and the criteria from the National Institute of Neurological and Communicative Disorders and the Alzheimer's Disease and Related Disorders Association (NINCDS-ADRDA)[207]. The DSM-III-(R) criteria for PPD require the presence of dementia, an insidious onset with a generally progressive deteriorating course, and the exclusion of all other specific causes of dementia by history, physical examination, and laboratory tests. The criteria may thus also include other relatively rare disorders, such as Pick's disease, Lewy body disease, and the dementias with non-specific brain changes (referred to as the ' $5 \%$ problem' [158]).

The NINCDS-ADRDA criteria are compatible with those of the DSM-III-R, but are more elaborate and better operationalized. For this reason, they are preferred in most studies. The criteria differentiate between possible, probable, and definite $\mathrm{AD}$. A diagnosis of definite $\mathrm{AD}$ can only be made when the criteria for probable $\mathrm{AD}$ are fulfilled, and when there is histopathological evidence obtained from biopsy or autopsy. Probable $\mathrm{AD}$ is defined by the presence of dementia, an onset between 40 and 90 years of age, and the absence of systemic disorders that in and of themselves could account for progressive deficits in memory and cognition. A diagnosis of possible $\mathrm{AD}$ is made when there are variations in onset, presentation, clinical course or in the presence of a second possibly dementing disorder, which is clinically not considered to be the cause of dementia. Allowance is made for plateaus in the course of progression or in the presence of associated psychiatric, somatic, or neurological symptoms.

The interobserver reliability of the NINCDS-ADRDA criteria is satisfactory, with kappa values of about $0.65[175,194]$. Studies of the validity of the clinical diagnosis of AD from before the NINCDS-ADRDA criteria generally reported a poor accuracy of the clinical diagnosis of $\mathrm{AD}$, with sensitivity and specificity rates of $28 \%$ and $43 \%$, respectively $[214,311]$. In contrast, various studies of the validity of the diagnosis of AD when the NINCDS-ADRDA criteria were used reported sensitivity rates between 64 and $95 \%$ and specificity rates ranging from 81 to $100 \%[26,143,215,310]$. The present evidence is that the clinical diagnosis of $A D$ corresponds with the pathological diagnosis in about $80 \%$ of the cases. It can therefore be concluded that the modern criteria have elevated the accuracy of the clinical diagnosis of $\mathrm{AD}$ to a highly satisfactory level. However, some remarks can be made here on these studies. First, there is considerable variation in the 'gold standard' of the criteria (i.e., neuropathological verification) among different pathologists [76, 142, 162, 237]. Moreover, not all patients who have the typical pathological hallmarks of Alzheimer's disease are per se demented [60]. The alternative way of validating the criteria of $\mathrm{AD}$ in the absence of pathological data is by 
documentation of a continuous progressive course [270], but this method cannot differentiate between $\mathrm{AD}$ and other progressive neurodegenerative disorders. Second, data on pathological validation come almost exclusively from carefully selected samples of patient groups. Most studies have examined relatively young patients from university centres without significant comorbidity. Thus, the results of these data cannot be superimposed on the typical geriatric population without due consideration. Third, most of these validation studies pertain to material obtained from patients in the terminal stages of dementia, a condition that is much easier to diagnose. Therefore, the impact of the results from these validation studies may be more limited in groups of patients who are in earlier stages of their disease.

Another problem is that $\mathrm{AD}$ is largely a diagnosis formed by exclusion of other diagnoses. The only two inclusion criteria are the presence of dementia and a gradual progressive course $[7,207]$. Thus, the accuracy of the diagnosis of $A D$ is directly related to the accuracy with which other diseases are excluded. Consequently, uncertainties in the diagnosis of other aetiologies, such as vascular dementia (vide infra), carry immediate consequences for the diagnosis of $\mathrm{AD}$.

\section{Definition of vascular dementia}

Vascular dementia (VD) is a syndrome of cognitive impairment resulting from vascular diseases, especially ischaemic brain changes. Hardening of the arteries has long been regarded as the single cause of dementia and the issue of defining VD has received appropriate attention only recently. VD is the second most common cause of dementia, accounting for about $20 \%$ of all dementias whereas combinations of VD and AD occur in another $10-15 \%[84,158,313]$. Recent studies with 85 -year-old patients have suggested an even higher prevalence of VD [286]. Previously, it was thought that the most important determinant of cognitive deterioration was the total volume of infarcted brain $[84,313]$. However, the total size of the infarcts is often small, and the cognitive deterioration appears to be also closely related to the side, site, and number of infarcts $[79,85]$. Athough the condition is often named "multi-infarct dementia", repeated infarctions in the brain are but one of the mechanisms leading to VD. Therefore, the term multi-infarct dementia should be avoided. The other cerebrovascular mechanisms include: dementia after one single stroke, small vessel disease, hypoperfusion of the brain, intracerebral haematoma, subarachnoid haemorrhage, inflammatory arteriopathies of the brain vessels, and other non-hereditary and hereditary vascular diseases [85].

The most widely used clinical criteria for vascular dementia, those of the DSM-III and DSM-III-R, are based on clinical description $[6,7]$. The features are not defined in detail and leave much room for interpretation (e.g., 'patchy' distributions of deficits); brain-imaging data are not incorporated. Ischaemic scales have long been used for the diagnosis of VD [117, 192, 269]. More recent criteria have also paid attention to the temporal relationship between dementia and strokes, the number of strokes, and brain-imaging data $[46,266]$.

Current criteria for the clinical diagnosis of VD share the same definition of dementia as those of other aetiologies. This raises the question whether there are phenomenological differences between the various dementing disorders. For instance, depression, retained 
insight, and preserved personality have all been reported to occur more frequently in VD than in $\mathrm{AD}[87,118,199]$. However, there is still little empirical evidence that the two types of dementia can be accurately differentiated on clinical grounds. The cognitive syndrome in advanced $\mathrm{VD}$ may be different from that of $\mathrm{AD}$, but virtually no data exist on the clinical features of VD in the early stages [12].

\section{Phenomenology of the prodromes of dementia}

The onset of $\mathrm{AD}$ is often dated in retrospect and with imprecision. The reports from relatives can provide insight into the very first prodromes, but are largely anecdotal. A fine and instructive description was published in 1950 by a colleague of a medical scientist [9]:

"Over the period that we worked together, I gradually became aware that the fine edge of his intellect was becoming dulled. He was less clear in discussion and less quick to make the jump from a new piece of evidence to its possible significance. He spent more time over his work and achieved less; and he found it increasingly difficult to get his results ready for publication. He tended also to become portentous and solemn about his subject, as though one small comer of knowledge nearly filled his world, and the wider horizons were narrowing in. The change was so slow as to be barely perceptible, and the signs vanished when I tried to pin them down: they were like those faint stars which are seen more easily when they are not in the direct line of vision....To me it was as though a light had gone out, but no-one else seemed to notice anything amiss... By this time he was worried about his general health and attended a doctor from time to time with rather vague symptoms. For several years he had been said to have low blood pressure, but nothing was found wrong apart from this, and he was reassured."

Descriptions such as this are convincing and recognizable for clinicians. A similar clinical picture can be distilled from longitudinal studies and from retrospective studies making use of relatives' accounts. Typically, predemented subjects demonstrate feelings of anxiety, worrying, depression and psychological vulnerability. These feelings are less pervasive than in patients with major depression $[1,238,274]$. Their inner feelings may be more dependent on their environment: they calm down when reassured, but a small amount of stress is likely to put them out of balance $[1,238]$. They get exhausted easily. Passivity, lack of interest, and coarseness may be common features. They appear less spontaneous and extrovert, and more withdrawn. Some mental features of this syndrome reflect a disruption of compensating abilities, such as the rapid exhaustion and the difficulty to adjust to events that are not part of a daily routine, whereas others are more likely to be secondary phenomena, such as withdrawal, anxiety, and the tendency to worry $[29,41,238,240,275]$. Predemented patients themselves may or may not complain about these changes, but their relatives are likely to do so $[25,43,204,226,234,242]$. Neurological examination probably does not reveal any abnormalities nor do ancillary investigations such as laboratory or neuroimaging data [103, 107], although there may be an increase in 'soft' neurological signs early in the course of the illness [326]. Predemented subjects may perform within normal ranges on traditional psychometric tests of memory, but they are likely to need more time and show the tendency 
to get tired and to slow down when testing takes too long $[124,140,299,300]$. If memory is impaired, then it is usually related to tasks measuring delayed recall $[1,238,276,300]$.

The picture of predementia as is presented here should be regarded as tentative. It is not known if all patients with $\mathrm{AD}$ present with a picture similar to the one described here. Moreover, we do not know its predictive value in terms of specificity. To date, no clear diagnostic categories adequately classify the above clinical picture. The state may be too subtle to be described by reliable research criteria. A DSM-III-R diagnosis of dysthymia may be appropriate when the period of duration is longer than two years [7]. Patients who have symptoms of shorter duration will not receive any diagnosis according to DSM-III-R. The clinical picture of the prodromal stages shows similarities with concepts that used to be popular in the past, such as neurasthenia [141] or 'Vorseitige Versagenszustände' (premature states of failure) [20]. A qualitative approach, i.e., a detailed psychopathological description, is probably the most adequate. The importance of a qualitative assessment is underlined by the relative lack of specificity of the traditional psychometric tests [299].

\section{The classification of mild cognitive syndromes}

In this section, several diagnostic concepts and criteria used for the classification of mild cognitive syndromes are reviewed. At present, there is no consensus as to which concept is the most valuable. The main reason for this is the lack of longitudinal data which could provide data on their prognostic value. Nevertheless, the concepts provide a good starting point, acknowledging that more research still needs to be carried out. Only the descriptions of mild cognitive syndromes, including mild dementia, are discussed here, because the more severe stages are beyond the scope of this review. The criteria of the syndromes that are discussed here are shown in the appendix of this thesis.

Benign senescent forgetfulness (BSF)

The concepts of 'Benign Senescent Forgetfulness' (BSF) and 'Málignant Senescent Forgetfulness' (MSF) were introduced more than 30 years ago. They are probably the earliest attempts to distinguish dinically berween normal age-related memory changes in the elderly and those forms of memory change with a worse prognosis $[170,171,173]$. The term has often been used in the medical literature and has become a generally accepted notion among clinicians. BSF presents as "...patchy and variable, with difficulties remembering details of experiences (names and places), but with relative ease in recalling the experiences itself. Usually, the forgotten details are recalled later". The condition is not progressive and does not increase the risk of the subject developing dementia. In contrast, MSF is characterized by an inability of the subject to recall events in the recent past, disorientation with regard to personal data, and retrogressive loss of remote memories. Subjects with MSF remain unaware of their deficit and frequently produce confabulations. 
The description of BSF is based on observations of retirement-home residents with a mean age of nearly 80 years. From a nosological point of view, it is unclear whether BSF is different from normal ageing or should be seen as a distinct entity between normality and pathology (dementia). Kral never made any distinction between a normal group and his benign patients. He considered MSF as the 'axis syndrome of psychosis with senile brain disease' [171]. Thus, MSF was meant to describe pathology, but it is unclear whether the concept should be regarded as a synonym of what is now called dementia, as a part of it, or whether it should be differentiated from it.

BSF and MSF were never defined appropriately in operational terms. As a consequence, research data on the reliability of the criteria are sparse. Only few data exist on the prognostic value of BSF. Kral carried out a 4 -year follow-up study with 20 patients with BSF and 34 with MSF; only one patient with BSF declined cognitively, whereas all patients with the malignant form did [171]. Death rates in BSF and MSF also differed significantly from one another: $38 \%$ vs. $61.7 \%$ after a 4 -year observation period. Recently, a 3-year follow-up study of patients with supposed BSF was reported by O'Brien et al.[226]: six of the 68 patients with BSF (9\%) had become demented, which was about twice the expected rate. These latter findings cast doubt on the view that BSF always follows a benign course.

Because of the ill-defined criteria and their uncertain nosological status, the use of the terms BSF and MSF cannot be recommended for research and clinical purposes, in spite of their widespread popularity.

\section{Age-associated memory impairment}

The term 'Age-Associated Memory Impairment' (AAMI) was introduced by Crook et al. in 1986 [59]. AAMI denotes a condition in otherwise healthy middle-aged or elderly subjects (fifty years or older) who complain about memory loss and who score at least one standard deviation below the mean for younger adults on neuropsychological testing for secondary memory. The AAMI criteria were provisionally established in order to have a operational definition of normal cognitive ageing. Furthermore, they serve as a basis for selecting research subjects in order to study the epidemiology, course, and clinical significance of normal ageing, and to evaluate the effect of pharmalogical or other forms of intervention $[89,203]$.

The concept of AAMI was criticized recently for being too broad. It was also received with scepticism because of the possible attraction to the pharmaceutical industry of delineating a new disorder and thus creating an artificial market for products against ageing $[11,68,227]$. The usefulness of the concept can be doubted since the authors assume that "most of the people over 50 years may be affected by AAMI to some degree" [203]. However, the criteria provide a firm basis for research into memory changes related to normal ageing and, in contrast to BSF and MSF, AAMI has been defined operationally.

More specific remarks on the AAMI concept were made by Blackford and LaRue [23]. Their points of criticism included: omission of an upper age limit, omission of any means of quantification of subjective complaints, the criterion that "performance is one SD below the mean established for young adults on a standardized test for secondary memory", which 
precludes the use of tests developed especially for old populations, and the likelihood of meeting the above-mentioned criterion when more tests are administered. Besides, the AAMI criteria provide a minimum for the deviation in memory performance (at least one standard deviation), but no maximum, although dementia should be ruled out. Blackford and LaRue [23] suggested the use of a battery of four or more tests of secondary memory and added to AAMI the following new categories: Age Consistent Memory Impairment (ACMI), for performance within $\pm 1 \mathrm{SD}$ of the mean established for age on $75 \%$ or more of the tests administered, and Late Life Forgetfulness (LLF) to denote performance between 1 and 2 SD below the mean established for age on $50 \%$ or more of the tests administered. These modifications can be regarded as an interesting elaboration. Data on the reliability or validity of the AAMI criteria have not been published yet.

In summary, the AAMI criteria were meant to denote a normal state related to ageing, but they do not preclude a progressive decline per se. To date, no longitudinal data have been reported and therefore the prognostic significance of the AAMI concept remains unsettled. The use of the term AAMI in clinical practice is limited. Nevertheless, the criteria are well defined operationally and provide an excellent starting point for further scientific research. The recent adaptations of Blackford and LaRue (ACMI and LLF) warrant further evaluation.

\section{DSM-III-(R) criteria: mild dementia}

Neither the DSM-III, nor its revised version, contains criteria to classify mild cognitive impairment under the level of dementia [7]. The DSM-IV will include a new category termed 'Mild cognitive changes (subthreshold)' as part of the class of 'Cognitive impairment disorders' $[248,292,293]$. In contrast to the first version of the DSM-III, the DSM-III-R specifies the severity of dementia in terms of functional capacity (see table) [7].

The DSM-III-(R) criteria of mild dementia have proved to be reliable [99]. Their validity has been examined in a 1-year follow-up study with 79 mildly demented, but otherwise healthy $\mathrm{AD}$ patients [138]. All patients who were initially diagnosed as $\mathrm{AD}$ received the same diagnosis at follow-up, indicating a sensitivity of the clinical diagnosis of $100 \%$. In addition, the clinical diagnosis of $\mathrm{AD}$ appeared to be more sensitive than neuropsychological testing or administering the MMSE. Another follow-up study with $55 \mathrm{AD}$ patients (28 with mild dementia, 22 with moderate dementia and 5 with severe dementia) from several teaching hospitals yielded comparable rates [99]. However, the outcome was less favourable in a recent prospective community study with 87 mildly and moderately demented subjects with a DSM-III diagnosis of the Alzheimer type or Vascular dementia [224] . Of the 48 patients (33 with $\mathrm{AD}$ and 15 with vascular dementia) who could be tested after a period of three years, no less than 20 patients $(11 \mathrm{AD}, 33 \%)$ did not show intellectual deterioration and cognitive decline remained doubfful in another 11 patients ( 9 with $A D, 27 \%$ ). Thus, it can be concluded that the prognostic value of the DSM-III criteria for the mildest stages of dementia is high when applied by experienced clinicians to selected patient groups. In field studies, however, the criteria appear to be less accurate. 


\section{Global deterioration scale: very mild and mild cognitive decline}

In 1982, Reisberg and coworkers [255] presented the Global Deterioration Scale (GDS) for the grading of primary degenerative dementia. The GDS is to date one of the most widely used clinical instruments to stage the course of $\mathrm{AD}$. The scale describes cognitive and behavioural changes in seven stages. Stage 1 describes normality (no complaints, no deficits), stage 4 meets the (DSM-III) criteria for dementia, and stage 7 describes the terminal phase of dementia in which the patient is bedridden and mute [257] (see also the appendix of this chapter). Reisberg et al. stressed in their original paper that clinicians should be aware that the GDS presupposes accurate clinical diagnosis of the syndrome and that symptoms characteristic of stages 2 and 3 do not necessarily imply the onset of $\mathrm{AD}$ [255]. In fact, the absence of any objective deficits in employment or social situations in stage 2 is contradictory to the diagnosis of dementia as defined by the DSM criteria. As a consequence, patients with $\mathrm{AD}$ can only be classified as 'GDS 2 ' in retrospect and, vice versa, patients who receive a GDS score of 2 are by definition not demented, and thus cannot be diagnosed as $\mathrm{AD}$. It is important to note this, because there is a danger that the mere use of the GDS implies a diagnosis of AD. The interrater reliability of the GDS is high (kappa values of 0.82-0.92) [101, 113]. Its criterion validity was demonstrated by significant correlations with psychometric measures, $\mathrm{CT}$ and PET scan parameters [101, 113, 255-257].

Considering the scale in more detail, however, the description of stage 2 appears to be rather non-specific. For example, the description only refers to "forgetting where one has placed familiar objects or forgetting the names of known persons" without specifying for example the impact of the complaints. Furthermore, only a clinical interview is required to exclude objective evidence of memory deficits. The characteristics of stage 3 are more specific, but they refer almost exclusively to memory or related cognitive domains. The GDS has been criticized for being too narrowly defined because it places too much emphasis on memory functioning $[124,140]$. Moreover, behavioural features may array themselves differently from the way they are positioned in the GDS [77].

The prognostic properties of the various stages have been established reasonably. In a 2 year-follow-up study, the 30 patients in stages 2 and 3 (16 and 14 patients, respectively) remained alive, well, and living at home [259]; only the patients in stage 3 of the GDS declined mildly on cognitive but not on functional assessment parameters. In another study, with a follow-up period of approximately 3.5 years, only two of 40 patients with GDS stage 2 demonstrated notable cognitive deterioration [254]. Five of the 32 patients (16\%) with a GDS score of 3 had a negative outcome, i.e., three patients worsened, one was hospitalized and one died; on the other hand, three patients showed improved social functioning. However, the neuropsychological parameters worsened in $72 \%$ of these patients after a 2 -year follow-up [96]. Of the 22 patients with GDS stage $4,72 \%$ went on to deteriorate in social functioning [254]. The patients of this study were healthy (except for dementia), did not suffer from depression and had Hamilton scores lower than 16. Recently, it has been reported that "50\% of the GDS 3 cases declined and received a diagnosis of $A D$ within several years" [88], but details of that study were not available at this time. 
In summary, the GDS provides a practical tool for staging dementia, ranging from normal to very severe dementia. The scale should be used in combination with other diagnostic methods and presupposes an accurate syndromal and aetiological diagnosis. The global nature of the scale is both its weakness and its strength. A GDS score of 2 reflects normal ageing in practically all patients, whereas GDS stage 3 represents borderline cognitive functioning. From the stage of GDS 4, a progressive course of decline is likely.

\section{Clinical dementia rating scale: questionable and mild dementia}

The Clinical Dementia Rating scale (CDR) was designed by the Washington Research group on Dementia [139] and rates the severity of dementia as questionable, mild, moderate, or severe (scores of $0.5,1,2$, or 3 ). The individual performance is rated separately in each of the following categories: memory, orientation, judgement, and problem-solving, community affairs, home and hobbies, and personal care. From these ratings, an overall CDR score is calculated according to a complicated algorithm.

The criteria carry high face validity and are based on a broad sample of cognitive performances in daily life. Memory performance in daily life is considered to be the central criterion, the other features as secondary. The interrater reliability and the construct validity of the CDR have been demonstrated [34]. The scale has been used in several longitudinal studies, which make the CDR probably the best tested rating scale for staging dementia to date $[16-18,276]$. One follow-up study with 43 patients rated as CDR 1 (mild dementia) reported that 41 patients progressed into more severe stages at a follow-up after 66 months $[17,18]$. Recently, more follow-up data were reported from 16 subjects with questionable dementia of the Alzheimer type (CDR 0.5) $[276,300]$. The patients were selected by experienced clinicians using strict inclusion and exclusion criteria. After 84 months of follow-up, 11 of the 16 patients (69\%) had $\mathrm{AD}$ verified by autopsy and/or progressed to a more advanced CDR stage. Subjects with questionable dementia differed from controls and from patients with mild dementia on several psychometric measures [300]. However, neuropsychological testing did not allow for a differentiation between those patients who progressed and those who did not, since objective levels of performance between the groups appeared to overlap extensively.

In summary, the CDR is a well documented scale for rating dementia in selected samples. The scale is more difficult to administer than the GDS. From stage CDR 1 , an outcome of deterioration can be reliably predicted, whereas stage CDR 0.5 reflects the borderline state.

\section{CAMDEX: minimal dementia and mild dementia}

The Cambridge Mental Disorders of the Elderly Examination (CAMDEX) consists of a standardized psychiatric interview with the patient and caregiver, and a brief neuropsychological battery (the CAMCOG) $[271,272]$. The CAMDEX was especially designed for the detection of early dementia and describes four syndromes as guidelines for the staging of dementia: minimal, mild (early), moderate, and severe dementia. In contrast to the GDS 
and the CDR, the CAMDEX can be used to obtain a syndromal and an aetiological diagnosis. The criteria should be used in a "flexible manner", leaving "some room for clinical judgement" [271]. The face validity of the criteria is high. The interrater reliability of the CAMDEX is good [272].

Until now, no studies using the CAMDEX with selected groups of patients have been published. O'Connor et al. applied the CAMDEX in two longitudinal community studies in Cambridge on the same cohort, with a follow-up period of 12 and 24 months $[232,233]$. The subjects had been examined in a two-stage design, by which only those with a score of less than 26 on the Mini-Mental State Examination [97] were assessed further with the CAMDEX. It should be noted that the Cambridge cohort involved a more "geriatric" sample, i.e., the patients were older and less selected for absence of somatic or psychiatric disorders than the above-mentioned studies with the GDS and the CDR. This was reflected in a high overall mortality rate: from 44 subjects diagnosed as minimally demented, 11 patients died and only 29 were able to be reviewed after the first year of the study; only 6 of them showed progression of cognitive impairment, while 13 patients were judged to be normal [232]. However, after one more year of follow-up, 12 of the 24 retested subjects had progressed to dementia [233]. The authors concluded that although the CAMDEX criteria were in general feasible, the criteria for minimal dementia were more difficult to apply in clinical practice $[229,232]$. Errors in the diagnosis of minimal dementia occurred especially in people of a below-average intelligence, in the old and frail, and in patients with sensory impairments. The need for repeated assessments over lengthy periods was stressed in order to document the natural history of dementia correctly. The Cambridge follow-up study provides an illuminating example of the practical problems of community surveys and gives much insight into the differences between an epidemiological approach and approaches using carefully selected and evaluated clinical populations such as those described above [229].

\section{Comparison of the different criteria for mild cognitive syndromes}

When evaluating the prognostic significance of the different proposals for the borderline states between normal forgetfulness and frank dementia, it is important to note that hardly any of the criteria of the above-mentioned studies have been used in their own right: in most of the studies the diagnoses were made on the basis of a thorough investigation by experienced clinicians (e.g., [276]). In these studies, the criteria were used for the staging of severity, not for the diagnosis of $\mathrm{AD}$ itself. Only the studies with the CAMDEX were carried out without any other forms of assessment $[232,233]$. Comparison of the different concepts and criteria discussed above reveals a considerable overlap in clinical characteristics. At the outset, there are few differences between Kral's benign forgetfulness and Reisberg's very mild cognitive decline (GDS 2). Likewise, stage 3 of the GDS closely resembles the description of very mild cognitive impairment of the CDR (CDR 0,5) and the CAMDEX criteria for minimal dementia, whereas GDS 4, CDR 1, and mild dementia of the CAMDEX are all highly compatible with the criteria for mild dementia defined by the DSM-III-R. However, the concepts differ in details 
$[102,219,302]$, and thus comparison of the results of these studies should be approached with some caution.

Table 3: Rates of progressive decline of patients rated according to current concepts of cognitive impairment or early $A D$.

Reference $\mathrm{N}$ follow- age $\mathrm{C} / \mathrm{F}$ \% with up (yrs) decline

(mths)

\begin{tabular}{llllllll}
\hline GDS2 & very mild cognitive dedine & {$[270]$} & 7 & 36 & $66-92$ & $\mathrm{C}$ & 0 \\
GDS2 & very nild cognitive decline & {$[254]$} & 40 & 42 & 68,7 & $\mathrm{C}$ & 5 \\
BSF & benign senescent forgeffulness & {$[171]$} & 20 & 48 & 80,5 & $\mathrm{C}$ & 5 \\
& & {$[227]$} & 68 & 36 & 67,2 & $\mathrm{C}$ & 9 \\
GDS3 & mild cognitive decline & {$[254]$} & 32 & 42 & 71,1 & $\mathrm{C}$ & 16 \\
CAMDEX & minimal dementia & {$[232]$} & 29 & 12 & 80,3 & $\mathrm{~F}$ & 21 \\
CDR 0.5 & questionable dementia & {$[270]$} & 27 & 36 & $66-92$ & $\mathrm{C}$ & 26 \\
BSF & benign senescent forgetfulness & {$[241]$} & 6 & 24 & $?$ & $\mathrm{C}$ & 33 \\
CAMDEX & mild dementia & {$[232]$} & 67 & 12 & 80,3 & $\mathrm{~F}$ & 37 \\
DSM-III & mild and moderate dementia & {$[224]$} & 33 & 36 & 74,0 & $\mathrm{~F}$ & 40 \\
CAMDEX & minimal dementia & {$[233]$} & 24 & 24 & 80,3 & $\mathrm{~F}$ & 50 \\
CAMDEX & mild dementia & {$[233]$} & 56 & 24 & 80,3 & $\mathrm{~F}$ & 50 \\
CDR 0.5 & questionable dementia & {$[276]$} & 16 & 84 & 71,7 & $\mathrm{C}$ & 69 \\
GDS3 & mild cognitive dedline & {$[96]$} & 32 & 24 & 71,3 & $\mathrm{C}$ & 72 \\
GDS4 & moderate cognitive decline & {$[254]$} & 22 & 42 & 72,3 & $\mathrm{C}$ & 73 \\
CDR 1 & mild dementia & {$[17]$} & 53 & 66 & 71,4 & $\mathrm{C}$ & 95 \\
DSM-III & mild dementia & {$[99]$} & 27 & 12 & 74,4 & $\mathrm{C}$ & 96 \\
DSM-III & mild dementia & {$[138]$} & 79 & 12 & 67,4 & $\mathrm{C}$ & 100 \\
& & & & & & & \\
\hline
\end{tabular}

Note $\mathrm{C}=$ Clinical study; $\mathrm{F}=$ Field study

The results from the follow-up studies into the prognosis of the different criteria for early $\mathrm{AD}$ are summarized in Table 3 . On average, the patients were relatively young and the mean follow-up periods were short. Furthermore, the studies show considerable variation in the number of subjects, their mean age, and the duration of the follow-up period. In general, field studies were less successful in predicting dementia than those carried out in specialized dementia clinics. The diagnosis of mild dementia, more so than in the advanced stages, deals with a subtle interplay of disturbed behaviour, cognition, and affectivity, which are all complex concepts in their own right. Simple demarcations between normal ageing and mild dementia, as are necessarily used in epidemiological studies, cannot adequately cover "the wealth of clinical material that needs to be taken into account" [229]. This makes it hard to compare 
the studies with each other. With these conderations in mind, it can be concluded that the likelihood of further cognitive decline is low (but not zero) for subjects with mere memory complaints classified as BSF or GDS 2, intermediate for patients with questionable dementia, GDS 3 or CAMDEX minimal dementia, and high for patients with GDS 4, DSM mild dementia, CAMDEX mild (early) dementia and CDR 1 . Stated in another way, subjects who complain only about poor memory, without objective evidence of impaired memory and who perform well in daily activities cannot be regarded as being at high risk of becoming demented. In contrast, those who demonstrate objective cognitive impairments interfering with social functioning probably will decline. These statements are based on the results of studies with selected samples. In individuals with mild cognitive changes, it is still impossible to predict the outcome accurately.

\section{Clinical and methodological issues}

\section{The continuum between normal ageing and dementia}

When looking for the characteristics of mild dementia and, more specifically, of very early AD, it is important to discuss first what should regarded as normal. Usually, an operational, statistical definition of normality is used. Observations can be regarded as normal if they fall within some fixed boundaries, for instance, in the middle $90 \%$ of their range [132]. Rowe and Kahn recently stressed in an important theoretical review on ageing [273] that the variability of a given parameter, for instance, performance on a memory test, increases with age. The authors paid attention to the heterogeneity present even in normal, i.e., non-diseased, groups of elderly people. They argued that a distinction should be made between 'successful' and 'usual' ageing. Succesful ageing refers to changes that are intrinsic to age itself, whereas usual ageing is the result of ageing plus all non-pathological deficiencies that occurred earlier in life. These deficiencies are not intrinsically related to the process of ageing per se. However, they are still closely associated with ageing, because their effects are greater in old age (due to increased vulnerability or reduced resistance) $[132,149]$. In addition, the chance of being affected by these deficiencies increases with ageing.

The relevance of the distinction between successful and usual ageing was recently illustrated by Houx et al. [132-134], who demonstrated that the variability of various cognitive measures depends largely upon the sum of potentially health-threatening factors that occurred earlier in life. Examples of these factors, called Biological Life Events (BLE s), are mild brain injuries, repeated general anaesthesia, or periods of alcohol abuse. In a group of people who were all "normal" according to regular gerontological criteria, BLE-free subjects showed no increase in the variance of performance in memory and speed, whereas in those with one or more BLE s the age-associated decline was much more pronounced.

These observations are important when looking at the differences between dementia and normality. There is a growing body of evidence that suggests a continuum between normal and pathological cognitive decline. For instance, a study of randomly selected 70- to 79-year- 
old women yielded a smooth and unimodal distribution of cognitive functions and behavioural changes [32]. This does not, of course, imply that Alzheimer's disease is merely exaggerated ageing. Instead, it highlights the fact that differentiation in cross-sectional diagnoses is difficult. Therefore, in the absence of reliable biological markers, some uncertainties must be accepted in the cross-sectional diagnosis of very early $\mathrm{AD}$. Another consequence of the continuum is that different groups need different diagnostic criteria. Currently available norms have only been differentiated according to various age groups. An altemative approach would involve a more individualized assessment of early $\mathrm{AD}$. What is normal for one person, may not be normal for another. For instance, it may be relevant to develop other norms for those subjects with several BLE s as compared to those subjects without any.

\section{Non-cognitive bebavioral markers of early dementia}

Without exception, the current definitions characterize dementia as a state of cognitive failure. Indeed, there is good evidence that deficits of memory are among the earliest features of $\mathrm{AD}[138,268,334]$. However, this does not mean that impaired memory is a prerequisite for the diagnosis of $\mathrm{AD}$ in its prodromal stage. That which has to be proven is accepted as proof when memory impairment is tautologically considered to be the main characteristic of the preceding stages of dementia (e.g., [300]). A large amount of evidence from epidemiological and descriptive studies shows that non-cognitive symptoms are prominent in the disintegration of psychological organization. These non-cognitive symptoms involve perception, motility, personality organization, emotional experience, and volition (see [22] for review). Recently, Berrios has challenged the view that prodromes of dementia should be defined as a form of 'mini-dementia', i.e., only quantitively differing from dementia.

The most frequently studied non-cognitive aspects of early $\mathrm{AD}$ are changes in affectivity and in personality $[29,41,73,78,94,98,240,274-276]$. These are discussed below in more detail.

Depression preceding dementia

Depression can be related to dementia in several ways. First, severe depression may present with the picture of dementia. This condition has also been called 'pseudodementia' [163], although there is nothing 'pseudo' about it for those who adhere to the strict syndromal definition of dementia $[131,196,315]$. Second, depression and dementia may coexist [253]: depression can be then seen as an understandable psychological reaction to the growing awareness of an impending deterioration $[323,333]$ or as a result of biochemical abnormalities in the brain $[342,343]$. Third, depression may precede dementia $[172,252]$ : depression may thus be a very early symptom or subclinical dementia may become manifest under the stress of depression or treatment with antidepressive (often anticholinergic) drugs. Fourth, dementia, notably the subcortical types, can mimic depression [262]. And fifth, it is also possible that both conditions develop independently of one another. 
The notion of depression being an early manifestation of dementia is still under debate. In a retrospective study, relatives of patients with $\mathrm{AD}$ reported depression and agitation in about $40 \%$ as being the first symptom of dementia [181]. In prospective studies, depressive symptoms in elderly patients were also found to increase the risk of the patient developing subsequent dementia when present in combination with cognitive deficits $[172,252]$. However, other studies did not report an increased prevalence of depressive symptomatology in subjects who subsequently developed dementia $[157,238]$.

The results of these studies have to be evaluated carefully, because the definitions used for depression and dementia may be different from those adhered to today. For instance, in most of the studies, a favourable effect of antidepressant drugs was taken as proof of the presence of depression and of the absence of dementia. It is likely that the 'pseudodemented' patients were in fact patients with true dementia caused by Alzheimer's disease, but whose depressive presentation strongly biased the diagnosticians. Support for this notion comes from neuropsychological studies that found more differences between depression and depressive pseudodementia than between depressive pseudodementia and dementia $[78,98]$. Furthermore, most of the evidence of depression as a possible precursor of $\mathrm{AD}$ comes from studies carried out in departments caring primarily for demented or cognitively impaired subjects. This may reflect the referring physician's suspicion of organic cognitive impairment rather than functional psychiatric disorders. Therefore, the results may be biased on the basis of selection.

In summary, patients with depressive symptoms and cognitive deficits may relatively often have subclinical $\mathrm{AD}$. Unfortunately these patients have been rejected from participation in most studies on $\mathrm{AD}$, probably due to a dichotomous view in which depression and dementia are regarded as mutually exclusive. More refined clinical and neuropsychological descriptions and prospective neuroimaging and neurochemical studies should be carried out in order to elucidate the relationship between depressive symptoms and mild dementia.

\section{Personality changes preceding dementia}

Personality changes in relation to $\mathrm{AD}$ have been studied only recently. In a study carried out in the Maastricht Memory Clinic on the first symptoms of dementia reported by the caregivers, personality changes preceded the cognitive manifestations in 10 out of 24 patients with mild $\mathrm{AD}$ (Verhey, 1987, unpublished data). Most of the patients had been judged by their relatives as having become lazier, slower and more withdrawn. Petry et al. [240] compared the spouse ratings of the patient's current behaviour with ratings on personality as remembered prior to the appearance of any symptoms of $\mathrm{AD}$. They found that $\mathrm{AD}$ patients became more passive, more coarse and less spontaneous. The personality changes occurred early in the course of the disease and could not be attributed to the decline in intellectual function. Similar profiles (diminished initiative, loss of self confidence, decreased extroversion, growing apathy, relinquishment of hobbies and increased rigidity) have also been found in other studies $[1,29$, $41,238,274]$. Many subjects had subtle personality changes long before dementia became clinically manifest. These studies revealed a remarkable uniformity in the direction of 
personality changes (slower, less open, more withdrawn). This challenges the frequently claimed notion that personality changes involve an exaggeration of premorbid personality traits (eg, $[7,187])$.

One recent study merits special attention because of its longitudinal design: a nine-year prospective study, performed in Gotenburg, Sweden $[1,238]$. In this study, a sample of randomly selected 70-year-old subjects were examined by using psychological tests and personality assessments. After nine years, those subjects who subsequently developed dementia were compared with those who did not. The findings of the Gotenburg study confirmed the outcome of the above-mentioned studies, namely that personality changes occur early in the course of $\mathrm{AD}$. Moreover, it appeared that the time needed to complete several cognitive tests and the personality dimension pertaining to mental energy predicted the development of dementia. Mental energy was assessed by questions concerning energy resources, attitude towards new and difficult tasks, ability to change from one task to another and a tendency to get stressed and worried. The psychiatric interview revealed significantly more often a history of loss of interests and self-confidence and difficulties in making decisions in those subjects who subsequently deteriorated.

Thus, personality changes are likely to be among the earliest features of $\mathrm{AD}$. Although the assessment of personality in demented patients presents unusual and difficult methodological problems, personality alterations may be seen as a marker of early $\mathrm{AD}$ and warrant further investigation.

\section{Subjective cognitive complaints as an early marker of dementia}

With respect to the early detection of dementia, it is important to know whether the judgement of ones own memory (often referred to as metamemory $[245,284]$ ) reflects objective cognitive functioning and whether memory complaints have diagnostic meaning. Complaints of memory are quite common in the elderly and in middle-aged subjects. Difficulties in remembering were reported by no less than $73 \%$ of the 40 -year-old and by $91 \%$ of the 70-year-old subjects [25]. Cognitive complaints are associated with normal ageing, organic brain diseases, and a variety of other disturbances such as metabolic disorders, use of drugs, or affective disorders. Besides this, the growing interest of the media in dementia and Alzheimer's disease may have increased the awareness of memory deficits as a possible first sign of mental deterioration [318]. Although the majority of people who complain about this do not regard a decreased memory as a severe handicap [303], some of them do experience these complaints as distressing and this warrants their further examination.

A lot of evidence suggests that memory complaints in older adults correlate to a large extent with depression, but only modestly with their performance on objective memory tests $[25,43,204,234,242,303]$. However, the finding that the association of subjective complaints to depression is stronger than to dementia should not be interpreted too dichotomous manner since depression may also be a presenting symptom of Alzheimer's disease [252]. Thus, metamemory seems to be a poor predictor of dementia. However, a recent study reported that the relatives' assessments of the patients' memory, measured by 
standardized questionnaires, was a reasonable predictor of dementia, with an overall classification accuracy rate of $74 \%$; this rate was improved by $11 \%$ with the addition of objective memory tests [204]. These findings underline the diagnostic importance of taking a history from a relative or other informant who can report on the premorbid behaviour of the patient. The value of this has not been investigated sufficiently so far in clinical research. Although metamemory may be a poor predictor of cognitive decline in patients who are already demented, it would be of interest to investigate whether complaints of one's decreasing cognitive functions may anticipate manifest dementia.

\section{Who does the assessing?}

Dementia is generally recognized as a problem that requires a multidisciplinary approach [179, 290,308 ], but in clinical practice the diagnosis is usually made by one discipline. The several specialties involved in the field of psychogeriatrics often regard their own discipline as essential. For example, at the $\mathrm{CBO}$ consensus meeting on dementia in the Netherlands (Utrecht) in 1988, each of the representatives from neurology, psychiatry, neuropsychology, and geriatric medicine stated that patients with dementia should be examined by their own discipline [40]. A meta-analysis of the literature on the prevalence of dementia recently showed that specialty of the person making the assessment (e.g., general practitioner, psychiatrist, neurologist, or geriatrician) is an important methodological variable accounting for interstudy differences in the prevalence of dementia [153]. In view of the importance of clinical judgement in the assessment of mild dementia, it is remarkable that so little attention has been paid to which discipline is the best equipped to make this diagnosis. Will every patient indeed benefit from a diagnostic work-up by all above-mentioned specialities? Or is it possible to determine specific problem areas that require the expertise of specific specialists? These questions are still unanswered.

\section{Neuropsychological assessment}

Neuropsychological tests can make the diagnosis of mild dementia considerably more certain $[124,140]$. There are many neuropsychological tests [183], but special demands are made when they are applied to the diagnosis of mild dementia, e.g., the tests should not be too difficult or time-consuming, and appropriate norms must be available for subjects of different ages [140]. Several approaches for the assessment of $\mathrm{AD}$ have been proposed, including traditional psychometry [299], approaches using the information-processing paradigm [30, $155,297]$, behavioural neurology $[81,301]$, and combinations of these three [146]. Subjects with mild dementia and healthy controls differ on a number of neuropsychological functions, including aspects of memory [81, 86, 114, 265, 299, 334, 335], word fluency [299], spatial abilities [140], and other functions $[145,146,212]$. Memory impairment is a prominent feature of dementia and is regarded as essential for the diagnosis [7, 207]. In particular, measurements of delayed recall have been reported to have a high sensitivity and specificity in the diagnosis of early $\mathrm{AD}[67,265,335]$. Unfortunately, no consensus has been reached so far 
on the choice of neuropsychological tests. The NINCDS-ADRDA criteria for $\mathrm{AD}$ have determined the different cognitive domains to be examined and only suggested some commonly used tests. More recently, however, efforts have been made by the Consortium to Establish a Registry for Alzheimer's Disease (CERAD) to standardize the assessment of cognitive functions $[217,218]$.

Much emphasis is traditionally laid on memory functions in all neuropsychological approaches, but memory loss is not the sole basis for a diagnosis of dementia. By definition, demented subjects also have deficits in cognitive domains other than memory $[7,138,207]$. In particular, tests for language (fluency, naming) have found to be valuable in the assessment of early $\mathrm{AD}[138,217,334,335]$. A broad neuropsychological approach including tests that assess language, praxis, attention, visual perception, and problem-solving is recommended for the diagnosis of $\mathrm{AD}$ [207]. Although memory tests alone differentiate reliably between selected patients with mild $\mathrm{AD}$ and healthy subjects $[81,86,114,239,299]$, diagnostic errors can be expected when the tests are used in unselected populations, e.g., in alcoholics, depressives, or physically ill subjects $[10,49]$. The performance of mildly demented subjects on traditional psychometric tests shows considerable overlap with that of normal subjects, possibly due to a lack of consideration for individual aspects, such as premorbid intelligence, anxiety, sensory impairments, or physical disorders. In addition, genuine memory deficits have to be sorted out from apparent deficits. These apparent deficits can be due to ineffective strategies, impaired attention, reduced processing capacity, fear of failure or impairment of other cognitive processes that influence memory [247]. These psychological aspects have to be taken into account before a reliable judgement on memory functioning can be made.

Therefore, clinical judgement cannot be missed and is probably more sensitive to early $\mathrm{AD}$ than psychometric methods $[138,274]$. Thus, although neuropsychological tests provide an essential cornerstone to the diagnosis of mild dementia, they should be integrated with other types of assessment, notably those from psychiatry and neurology. A combination of the two, clinical assessment and psychometric tests, with techniques based upon different paradigms is the most accurate approach to date.

\section{Bedside cognitive rating scales}

The use of brief screening tests to obtain an objective measure of the mental status has increased dramatically over the past decades. The major advantage of all structured screening tests over unstructured bedside examinations is that they provide standardized methods of data collection and interpretation and thus improve diagnostic accuracy. A variety of different screening tests exist, of which the Mini-Mental State Examination (MMSE) is definitely the most widely used [97]. The MMSE serves as an example of the feasibility of other similar screening instruments as well $[90,91,130,222,246]$, but it should be noted that differences exist between the several scales. However, a detailed discussion of these differences is beyond the scope of this review.

The MMSE was originally designed to differentiate organic from functional psychiatric patients. Nowadays, it is largely used with demented subjects as a diagnostic tool in 
epidemiological and clinical studies for assessing the severity of cognitive impairment and to document cognitive changes over time. The psychometric properties of the MMSE are well documented. The scale has moderate-to-high levels of reliability. Furthermore, its score correlates highly with the scores obtained with other screening instruments, indicating good construct validity; its sensitivity is satisfying in moderate and severe dementia. In addition, the scale can reliably document cognitive changes in longitudinal research with dementia patients (see [312] for an overview).

However, there are also serious limitations to bedside screening instruments. For instance, the MMSE has been criticized because it is highly verbal and it does not pay enough attention to the measurement of visuospatial praxis. Therefore, it cannot detect focal lesions in the right hemisphere. However, the most important shortcoming of all bedside rating scales is their lack of sensitivity to mild cognitive impairments and their failure to discriminate between mild dementia and normal ageing. For instance, applying the traditional cut-off score of the MMSE (between 23 and 24) to patients with mild dementia results in a false-negative outcome in about half of the patients $[106,138,165,246]$. Similar figures were found for other screening tests $[90,91,222,246]$; no dementia screening test is superior in this respect [130]. In addition, many screening tests are influenced by the patient's race and level of education [ 90 , 222].

Thus, bedside screening tests are inaccurate in the diagnosis of mild dementia in individual patients. Bedside tests do not increase the level of diagnostic accuracy achieved through clinical examination alone [312]. However, administering the MMSE, or similar scales, may be of use to standardize global cognitive assessment, to document a clinical impression of cognitive dysfunctioning, and to obtain a baseline measurement for longitudinal evaluation in daily practice.

\section{Clinical beterogeneity of the dementia syndrome}

Dementia is currently defined in an identical way as many different diseases. The DSMIIL-R definition allows for considerable variation in the clinical picture [7]. The sine qua non in this definition is the combination of memory impairments and disruption of social functioning. Furthermore, patients with dementia may or may not have impaired abstract reasoning, disordered judgement, aphasia, apraxia, agnosia, or personality changes. This raises the question whether there is only one type of dementia. There have been several attempts to classify dementia in subgroups according to different patterns of cognitive impairments and to the underlying pathology. The major divisions are between cortical and subcortical, and between frontal and non-frontal.

The subcortical type of dementia was described for the first time in 1974 by Albert et al. [3] in patients with supranuclear palsy (SNP). Subsequently, other neurological disorders have been viewed as being typically subcortical: Huntington's disease, Parkinson's disease, Wilson's disease, lacunar state, normal pressure hydrocephalus, and AIDS $[15,61,72,206]$. Subcortical dementia is characterized by forgetfulness, slowing of thought processes, impaired ability to 
manipulate acquired knowledge, alteration of personality, apathy, and depression. In contrast, amnesia, aphasia, apraxia, and agnosia are regarded as cortical features.

The frontal type of dementia has been described by Neary and coworkers [220, 221]. Its anatomical substrate is in the prefrontal regions of the cerebrum. The typical clinical picture of frontal dementia is characterized by personality changes such as impulsivity, apathy or coarseness, the loss of abstract attitude, perseveration, utilization behaviour and deficits in selective attention, arousal, and ability to screen out irrelevant stimuli $[184,185,195,209$, 221]. Pure amnesia and instrumental deficits (aphasia, apraxia, or agnosia) are rare, although anomia may also be part of the frontal picture. Many of these alterations lead to socially inappropriate behaviours, whereas the cognitive functions may still be relatively intact. A typical example of a disorder presenting with this frontal symptomatology is Pick's disease, although a frontal-type dementia has been described in patients who lack these typical pathological hallmarks of Pick's disease $[220,221]$.

Although the concepts of cortical, subcortical, and frontal dementia have considerably stimulated interest into classifications on the basis of brain-behaviour relationships, their validity remains uncertain. There is a considerable overlap in the clinical presentation of the different types of dementias and their differentiation on the basis of cerebral localization appears to be difficult [338]. Relatively few data exist on the features of the different types of mild dementia. In a recent retrospective study, the presenting symptoms of Alzheimer's disease and Pick's disease were compared [208]: personality changes, hyperorality, and disinhibition were reported to be more frequent in Pick's disease, but personality changes occur also early in the course of Alzheimer's disease [274]. Bradyphrenia and impaired memory recall are said to be early symptoms of subcortical dementia's $[44,71]$. Whether these clinical characteristics can differentiate between the different diseases in their early stages is a question that remains to be answered.

\section{Subgroups of Alzbeimer's disease}

A question related to the above-mentioned issue is whether Alzheimer's disease represents one concept or more. There is much evidence to suggest heterogeneity of Alzheimer's disease. Subtypes can be distinguished with regard to clinical features $[45,200]$, age at onset $[92,121]$, rates of deterioration $[125,296]$, brain-imaging parameters [279], histopathology [27], neurochemical parameters [148], and genetic aspects [160]. Some studies suggested a subgroup of slow or non-progressive $\mathrm{AD}[36,200,296]$. For instance, an early onset has often been associated with more prevalent and severe language disorders and a faster rate of progression than an onset after 65 years of age $[45,125,282]$. Until now, attempts to describe the earliest manifestations of $\mathrm{AD}$ have not paid much attention to the issue of heterogeneity. However, this aspect deserves more investigation as it implies that different subgroups may present with different symptoms. For instance, aphasia may be a more sensitive marker for early Alzheimer's disease in relatively young patients, whereas impaired visuoconstruction may be a more sensitive marker in elderly subjects. 


\section{Continuous decline as an early marker}

Alzheimer's disease is a disorder which is characterized by a gradually progressive decline in cognitive and behavioural functions [207]. The process of change may be more important than the absolute levels of functioning. For instance, the decrease of a few points on the Mini-Mental State Examination [97] within a given period may be more meaningful than the absolute scores, even when above the traditional cut-off point. The course of decline in $\mathrm{AD}$ has only recently been studied more systematically in demented patients $[36,105,325]$, but hardly any studies exist on the prognostic value of certain changes in non-demented subjects. Sophisticated measurements that are sensitive enough to detect small changes with time are needed. Some other questions have still to be answered. How long should the minimal length of the intervals be? Which cognitive and functional changes are among the earliest symptoms? Which characteristics are best suited to longitudinal assessment?

\section{Age and comorbidity}

It should be noted that most of the research into $\mathrm{AD}$ has been carried out in relatively young and healthy patients, although dementia is more prevalent among the older and more diseased population. Older populations show higher levels of comorbidity. The relevance of this aspect has been noticed by O'Connor [229], who was confronted in his field studies with particular diagnostic errors in very old subjects and those with significant comorbidity. Old patients are more difficult to investigate appropriately. Besides, many old patients live in nursing homes or other protected environments, where criteria for social and functional decline are harder to meet than in younger subjects who are still working [60]. Moreover, samples with a high age will inevitably suffer more from methodological flaws such as higher mortality figures and decreased attendance at follow-up. Therefore, data from studies of relatively young patients cannot directly be applied to the typically geriatric population. It seems likely that the early detection of $A D$ in young and healthy subjects requires a different approach compared to that in the older samples. These aspects have generally been neglected so far in research of the earliest stages of $\mathrm{AD}$.

\section{Conclusions}

A conclusion of this review of the literature is that there is no unequivocal and reliable method currently available for the individual diagnosis of $\mathrm{AD}$ under the level of clinically manifest dementia. The first manifestations of this disease are subtle and difficult to distinguish from other behavioural syndromes. Once the threshold of dementia - as defined, for instance, by the DSM-III criteria - is passed, the prognosis can be predicted quite accurately. So far, prodromes of Alzheimer's disease have not yet been adequately translated into reliable diagnostic criteria. This may be due to a number of factors. Perhaps, the instruments used (e.g., consensus criteria, psychometric tests) are not the proper ones. The traditional 'gold 
standard' of neuropathology is virtually unavailable in this area. Validation must come from careful documentation of a progressive decline, with adequate assessment tools and sufficiently long periods of follow-up. Compared to the enormous amount of research into dementia and $\mathrm{AD}$, the number of longitudinal studies of early $\mathrm{AD}$ is small. At present, little can be expected from epidemiological field studies which of necessity work with reduced sets of data, A more individualized approach is needed that takes into account possible confounders from various categories such as earlier biological life events [132, 149], psychological states such as depression, bereavement or anxiety, premorbid personality, social factors, old age, comorbidity and sensory impairment. A clinical phenomenological approach combined with quantitative psychometric tests is probably the most sensitive method for the diagnosis of early AD but has the disadvantage of subjectivity.

Much research remains to be done. Doctors should be made aware of the importance of the detection of early $\mathrm{AD}$ with regard to the development of possible therapeutic strategies in the future, and should be encouraged to refer subjects at risk to specialised multidisciplinary services. A next step will be the collection of clinical data of people suspected of being at risk. Refinement of the available clinical instruments has a high priority. As it is not yet known which features are especially characteristic, a broad description is needed based on methods derived from traditional psychopathology, psychometric tests and qualitative clinical measures. Psychopathological descriptions must include the (alterations in) affectivity, volition, and self-experience. A more standardized assessment of personality characteristics is also useful. The history of the patient's relatives should receive much more attention, as this has proved to be one of the most sensitive instruments for the detection of early changes to date. It certainly needs to become more standardized. The categories from the DSM-III-R are obviously too static to delineate the prodromal stages precisely. More dynamic diagnostic strategies are needed to improve the accuracy of the diagnosis of very early AD. Tasks derived from the information processing paradigm may provide more insight into the different elements of psychological processes [297].

Another step would be to establish the validity of this presumed syndrome of predementia or, perhaps, various clusters of symptoms. This can be done in longitudinal studies, with repeated measurements over time. However, we first need to know which symptoms are the best ones to be followed over time and what the minimal period of follow-up in the earliest phases should be. Other configurations of symptoms may be detected and found to be of relevance by means of statistical procedures such as cluster analysis. Construct validation can be acheived by using SPECT, MRI or other neuroimaging techniques. Syndromes or symptoms with a potentially predictive value can then be isolated and earmarked as the essential hallmarks of early Alzheimer's disease. It will only be possible to develop less extensive diagnostic procedures of sufficient validity once it is known which signs and symptoms are characteristic of early Alzheimer's disease. 



\title{
3
}

\section{Diagnosing dementia: different disciplines, different diagnoses ?}

\author{
An experimental study of interdisciplinary differences \\ of participants of a consensus meeting on dementia*
}

\section{Introduction}

The lack of clear diagnostic criteria for dementia has for a long time hampered research and patient care, but in the last decades much progress has been made. In 1980, the DSM-III criteria for dementia were introduced, with a recent revision in 1987 [6, 7]. In 1984, an expert workgroup of the National Institute of Neurological and Communicative Disorders and Stroke, and the Alzheimer's Disease and Related Disorders Association (NINCDS-ADRDA) reached consensus about the criteria for the clinical diagnosis of Alzheimer's disease, which is the most frequent cause of dementia [207]. Several guidelines for the diagnosis of vascular dementia have been proposed as well $[44,84,119,192,266,269]$, although these are adhered to less uniformly, as their validity is still debated. Usually, the diagnosis of vascular dementia is based on the ischaemic score of Hachinski or one of its modifications [117]. These criteria have been proposed tentatively, to be modified on the basis of new insights. In the last years, several studies have reported on the validity and reliability of these criteria. The inter-rater reliability and reproducibility of the NINCDS-ADRDA criteria appear to be satisfactory $[99,175]$. Their validity is also acceptable, with a clinico-pathological agreement rate of about $80 \%[26,215,261,310]$.

Although the criteria were originally designed for research purposes, they are now generally accepted as the basis for the diagnosis of dementia in daily patient care $[54,167$, 281]. However, although the merits of the criteria for scientific research are obvious, there are

"Translation and adaptation from: FRJ Verhey, LA Plugge, JJE van Everdingen, J Jolles. "Dementie: verschillende disciplines, verschillende diagnosen?" Tijdschrift voor Geriatrie en Gerontologie 1991;22:187-194. 
hardly any data about how the criteria are adhered to in clinical practice. A few studies have investigated possible differences between a referrer's diagnosis on one hand and that of a specialized academic centre on the other $[108,128,322]$. It appeared that dementia was both under- and overdiagnosed in quite a number of patients. However, the academic centres used data that differed both quantitatively and qualitatively from those of the referrer, because the centres had access to more diagnostic resources, such as neuropsychology and sophisticated brain-imaging techniques. Therefore, these studies do not allow examination of the extent to which the differences in diagnoses are related to differences in the use of the criteria.

This paper describes an experimental study into the way clinicians of different disciplines make their diagnosis. We carried out an inquiry among the participants of the Dutch Consensus Meeting on Dementia, which took place in Utrecht, the Netherlands, in November 1988, and which was organized by the Dutch Organization for Quality Assurance in Hospitals (CBO). The participants of this meeting were asked to give their diagnosis (or diagnoses) of ten written case reports, which contained all data required to reach the diagnosis of dementia and its aetiology. In contrast to the studies mentioned in the previous paragraph, all participants received the same set of data.

The first aim of this study was to assess whether there are any systematic differences in the diagnostic outcome between the various disciplines involved in the diagnosis of dementia.. Second, we investigated the influence of certain patient variables, such as degree of cognitive decline, upon the level of consensus and the agreement with a gold standard.

\section{Methods}

\section{Procedure}

All 458 persons who were registered for the $\mathrm{CBO}$ consensus meeting on dementia received a booklet with five case descriptions (set 1) two weeks before the meeting. An introductory letter and a form were enclosed, and participants were requested to write down the diagnosis (or diagnoses) for these case reports. The participants were also asked to answer questions concerning their age, discipline, years of experience with dementia, and number of hours spent weekly in the differential diagnosis and classification of patients with dementia. Participants were not asked explicitly to formulate their diagnoses according to certain guidelines, in order to avoid any reference to the consensus meeting and thereby possibly influencing diagnostic usage and terminology. One week after the meeting a second booklet with five other case descriptions was sent to all who responded to the first part of the inquiry (set 2). Participation in the inquiry occurred anonymously.

\section{Material}

The two sets of five case reports each were selected from the records of real patients from the Maastricht Memory Clinic, but to guarantee the patient's privacy, any information that might 
identify the patient was changed. The information was incorporated in the following paragraphs: introduction, medical history, as reported by the caregiver, psychiatric and neurological history, medical history, medication data, intoxication data, psychosocial data, daily functioning, physical examination, neurological examination, psychiatric examination, neuropsychological examination, blood examination and additional examinations (such as CT scanning, X-ray of the chest and electrocardiogram). The clinical data were presented without any reference to the interpretation of them, e.g., wording such as 'no abnormalities' or 'impaired performances' was avoided as much as possible. The two sets of case reports were comparable in the severity and the complexity of the cognitive disorder. Each case description contained enough data to make a diagnosis according to the DSM-III-R criteria for dementia and to those of the NINCDS-ADRDA for the diagnosis of Alzheimer's disease. Sufficient data were provided to determine a score on Hachinski's Ischaemic Scale [119] and on the Global Deterioration Scale [255], in order to obtain a measure of the probability of a vascular aetiology and the degree of cognitive decline, respectively.

\section{Gold standard}

To establish a reference to enable comparison of the diagnoses, the same ten case descriptions were presented a few weeks after the consensus meeting to an independent multidisciplinary committee of three renowned clinicians (a psychiatrist, a neurologist and a neuropsychologist) experienced in the differential diagnosis and classification of dementia. The three clinicians were given the opportunity to discuss the data thoroughly and to formulate the final diagnoses. The committee was asked to state the diagnoses at both the syndromal and the aetiological level. The committee took about 4 hours to establish the diagnoses. The diagnoses were used as the golden standard or Reference Diagnoses (RD). The committee reached a consensus for all patients, except for the aetiology of patients 4 and 6 : in these two patients there were two possible aetiological diagnoses. The Reference Diagnoses are summarized in Table 1.

\section{Analysis of the data}

In order to perform a meaningful quantitative analysis of the diagnoses, the results of the inquiry were classified according to the following two-step hierarchical system. First, a syndromal diagnosis was made, and then the aetiological diagnosis. At the syndromal level, the possibilities were dementia, cognitive disorder not labelled as dementia, no cognitive disturbances, no statement on cognitive functioning. At the aetiological level, the following categories were included: primary degenerative, cerebrovascular cause, neurological cause other than cerebrovascular, somatic/ internal cause, intoxication/ side-effect of drugs, e.g, major tranquilizers, depression induced, related to psychosocial factors, and no statement on aetiology. Differences in the terminology used were taken into account at scoring; thus, diagnostic statements such as Azheimer's disease, senile dementia, SDAT or primary dementia, were all coded as 'dementia, primary degenerative' and, likewise, statements such 
as vascular dementia, Multi-Infarct Dementia, Binswanger's disease, and atherosclerotic dementia were all coded as 'dementia, cerebrovascular cause'. Although the diagnosis of depression is usually adhered to in a syndromal way [7], it was used here in an aetiological sense, i.e., as a possible cause for cognitive deterioration (cf. depression-induced dementia).

Table 1. Golden standard" diagnoses made by a multidisciplinary expert committee.

\begin{tabular}{|c|c|c|}
\hline Set & $\#$ & Case description \\
\hline \multirow[t]{5}{*}{1} & 1 & $\begin{array}{l}74 \text {-year-old woman } \\
\text { syndrome: moderate dementia, with depression } \\
\text { aetiology: probable Alzheimer's disease }\end{array}$ \\
\hline & 2 & $\begin{array}{l}\text { 80- year-old woman } \\
\text { syndrome: moderate dementia, } \\
\text { aetiology: probable Alzheimer's disease; drug induced parkinsonism }\end{array}$ \\
\hline & 3 & $\begin{array}{l}\text { 80-year-old man, } \\
\text { syndrome: severe dementia, } \\
\text { aetiology: vascular dementia, drug induced parkinsonism }\end{array}$ \\
\hline & 4 & $\begin{array}{l}\text { 66-year-old woman } \\
\text { syndrome: mild dementia, } \\
\text { aetiology: possible Alzheimer's disease or depression induced dementia }\end{array}$ \\
\hline & 5 & $\begin{array}{l}\text { 71-year-old woman } \\
\text { syndrome: slight cognitive deficit } \\
\text { aetiology: CVA; adjustment disorder }\end{array}$ \\
\hline \multirow[t]{5}{*}{2} & 6 & $\begin{array}{l}\text { 62-year-old man } \\
\text { syndrome: slight cognitive deficit } \\
\text { aetiology: history of TLA }\end{array}$ \\
\hline & 7 & $\begin{array}{l}\text { 80-year-old woman } \\
\text { syndrome: severe dementia, } \\
\text { aetiology: probable Alzheimer's disease }\end{array}$ \\
\hline & 8 & $\begin{array}{l}\text { 86-year-old woman } \\
\text { syndrome: mild dementia, } \\
\text { aetiology: vascular dementia with depressive symptoms }\end{array}$ \\
\hline & 9 & $\begin{array}{l}\text { 72-year-old man } \\
\text { syndrome: mild dementia, } \\
\text { aetiology: possible Alzheimer's disease; possible subclinical CVA }\end{array}$ \\
\hline & 10 & $\begin{array}{l}\text { 67-year-old man } \\
\text { syndrome: moderate dementia, } \\
\text { aetiology: vascular dementia }\end{array}$ \\
\hline
\end{tabular}

Note: $\mathrm{CVA}=$ cerebrovascular accident; $\mathrm{TIA}=$ transient ischaemic attack 


\section{Results}

One-hundred and twenty-seven out of the 458 participants of the consensus meeting completed the pre-consensus questionnaire. Of these 127 respondents, 90 completed the postconsensus questionnaire. Five of these 90 respondents were not actively involved in health care; thus, the data of the remaining 85 participants were analysed. Various characteristics of these 85 respondents are shown by discipline in Table 2 . About $20 \%$ of the participants of a given discipline responded, and no significant difference was found between the response rate per discipline and the actual number of participants per discipline who attended the consensus meeting (Pearson chi-square, n.s.). The disciplines were also comparable with regard to the number of years of experience of the participants in health care, mean age, and the reported amount of time spent reading the syllabus by the $\mathrm{CBO}$ on the diagnostic dilemma's of dementia that was edited (ANOVA, n.s.).

Table 2. Number of participants ( $\mathrm{n}, \%)$ in CBO meeting and characteristics of respondents by discipline

\begin{tabular}{|c|c|c|c|c|c|}
\hline discipline & $\begin{array}{l}N(\%) \\
\text { attending the } \\
\text { meeting }\end{array}$ & $\begin{array}{l}N(\%) \\
\text { responding } \\
\text { lo inquiry }\end{array}$ & $\begin{array}{l}\text { years of } \\
\text { experience } \\
\text { in health care }\end{array}$ & $\begin{array}{l}\text { age } \\
\text { (mean) } \\
\text { meeting }\end{array}$ & $\begin{array}{l}\text { bours } C B O \text { syllabus } \\
\text { was read before }\end{array}$ \\
\hline neurologists & $100(22)$ & $24 \quad(26.5)$ & 17.1 & 43.8 & 2.2 \\
\hline psychiatrists & $57 \quad(12.5)$ & $13(14.5)$ & 11.9 & 40.3 & 2.5 \\
\hline psychologists & $66 \quad(14.5)$ & 10 (11) & 13.9 & 43.7 & 3.4 \\
\hline nursing home GP's & $133(29)$ & $26(29)$ & 13.1 & 40.1 & 2.9 \\
\hline other physicians & $74 \quad(16)$ & $12(13.5)$ & 12.1 & 39.8 & 2.75 \\
\hline others & $28 \quad(6)$ & $5(5.5)$ & & & \\
\hline TOTAL & $458(100)$ & $90 \quad(100)$ & & & \\
\hline
\end{tabular}

First, we investigated whether there was any difference in the level of consensus before and after the consensus meeting. This was examined separately for the syndromal and for the aetiological diagnoses. As a measure of the degree of agreement, the proportion of the number of pairs for which there was agreement relative to the possible pairs of assignments was used. The way this proportion of agreement was calculated is derived from the kappamethods of agreement for $k$ raters and $n$ objects [285], and is described in more detail in our previous article [243]. In order to examine the differences in pre-meeting and post-meeting consensus, a two sided test was used to compare the level of proportion of agreement of each discipline for the five case reports. First, we tested for differences among all participants together. There were no significant differences in the level of consensus in the ten case descriptions or any differences with regard to the aetiological diagnoses. When calculated for each discipline separately, we did not find any significant differences either. 


\section{Agreement with the Reference Diagnosis}

Having concluded that there was no significant difference in the level of consensus before and after the consensus meeting, we combined the results of the ten case descriptions for further analyses. We investigated the level of agreement between the respondent's diagnoses and the Reference Diagnoses (RD) made by the multidisciplinary committee. For each diagnosis that corresponded with the $\mathrm{RD}$, the respondent received one point, both with regard to the syndromal diagnosis and the aetiological diagnosis. Thus, 10 points could be scored for the syndromal diagnosis and 10 points for the aetiological diagnosis. In patients 4 and 6 , in which the multidisciplinary committee could not reach consensus on the aetiology, both alternatives were considered "correct". The degree of agreement with the RD for syndrome and for aetiology is shown in the figures 1 and 2 . First, the syndromal diagnoses of all 85 clinicians were analysed together: the mean score of agreement with the $\mathrm{RD}$ was $7.6 \pm 1.4$ (range 1-10). There were no significant differences between the disciplines, the different disciplines being equally accurate in diagnosing dementia. The mean agreement score with the $\mathrm{RD}$ for the aetiological diagnosis, calculated for the entire group, was considerably lower: $5.3 \pm 1.7$ correct diagnoses (range 1-8). Statistical analysis revealed the following significant differences (Waller Duncan K-ratio T test, $\mathrm{p}<0.05$ ) between the disciplines: the neurologists had more correct diagnoses (mean score:6.2) than the psychiatrists, the nursing home physicians, and the other physicians. The psychiatrists made less correct diagnoses than the neurologists and the psychologists, whilst the other disciplines had an intermediate number of correct diagnoses. No significant relationship could be found between the agreement with the $\mathrm{RD}$ and the severity of the cognitive syndrome as assessed by the GDS score. However, the number of diagnoses that were correct appeared to be significantly lower in the five patients in whom depressive symptomatology was present compared to the other five patients without signs of depression ( $\mathrm{T}$ test for paired samples). This difference was found for both the syndromal diagnosis $(4.00$ vs. $3.57, \mathrm{p}<0.05)$ and the aetiogical diagnosis $(3.32$ vs. $1.97, p<0.01)$.

\section{Interdisciplinary differences in the use of particular diagnoses}

Next, we investigated whether some disciplines used some diagnoses more than the other disciplines. The following results were significant (Waller-Duncan $t$ test, $p<0.05$ ): the psychiatrists and the nursing home physicians made a syndromal diagnosis without any aetiological specification the most often, whereas the neurologists made more aetiological diagnoses. The diagnosis Alzheimer's disease was made more frequently by the neurologists than by the other disciplines. The diagnosis depression was made more often by the psychiatrists. Intoxication or drug side-effects was used more often by the nursing home physicians and the other physicians than by the psychiatrists and the psychologists, whereas the neurologists had an intermediate position. There were no significant differences for the other diagnostic categories (i.e., internal somatic, cerebrovascular, neurological and psychosocial). 


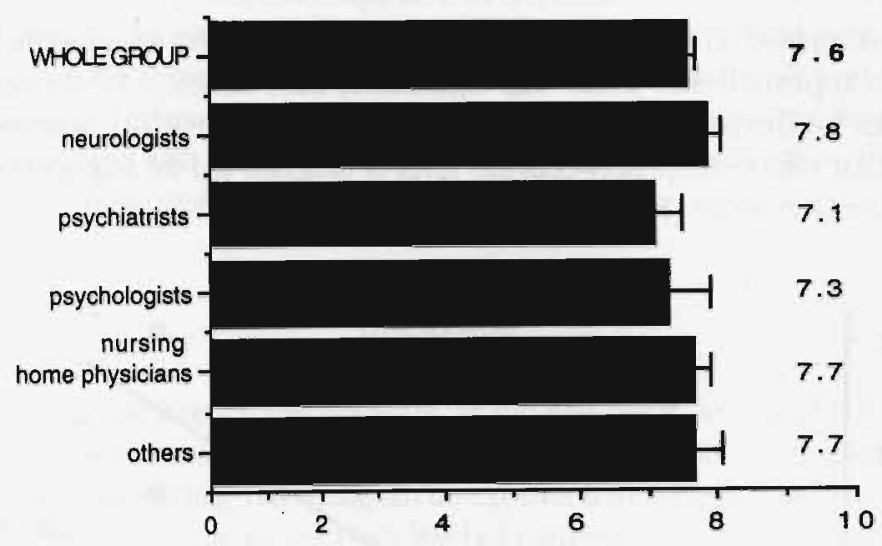

Figure 3.1 Mean number of syndrome diagnoses in agreement with the Reference Diagnosis

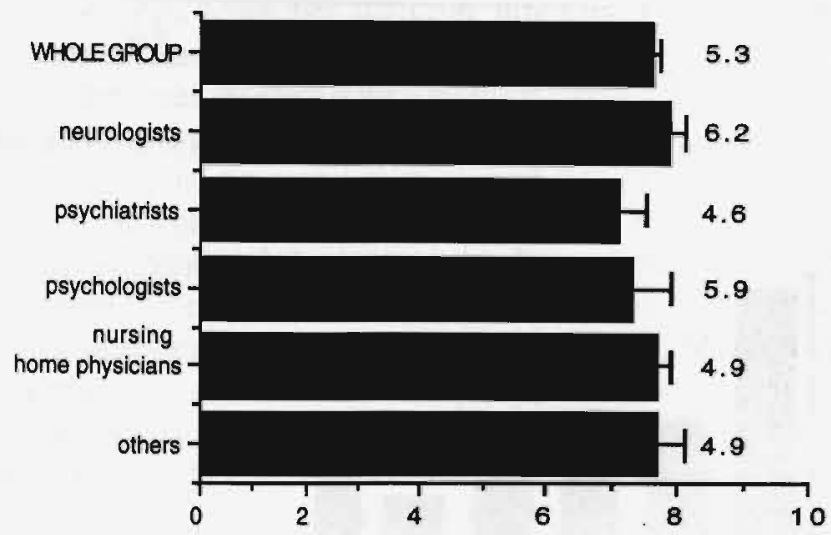

Figure 3.2 Mean number of aetiological diagnoses in agreement with the reference diagnosis. Significant were the following differences: neurologists vs. psychiarrists, nursing home physicians and other physicians; psychologists vs. psychiatrists. 
Factors of influence on the level of consensus

Finally, we investigated whether we could identify certain factors that influenced the level of consensus. It appeared that there was significantly more consensus for the syndromal diagnoses than for the aetiologic diagnoses (two-sided sign test, $p<0.001$ ). Moreover, there was a significant relationship between the level of consensus and the degree of cognitive decline, i.e., the GDS score (Figure 3).

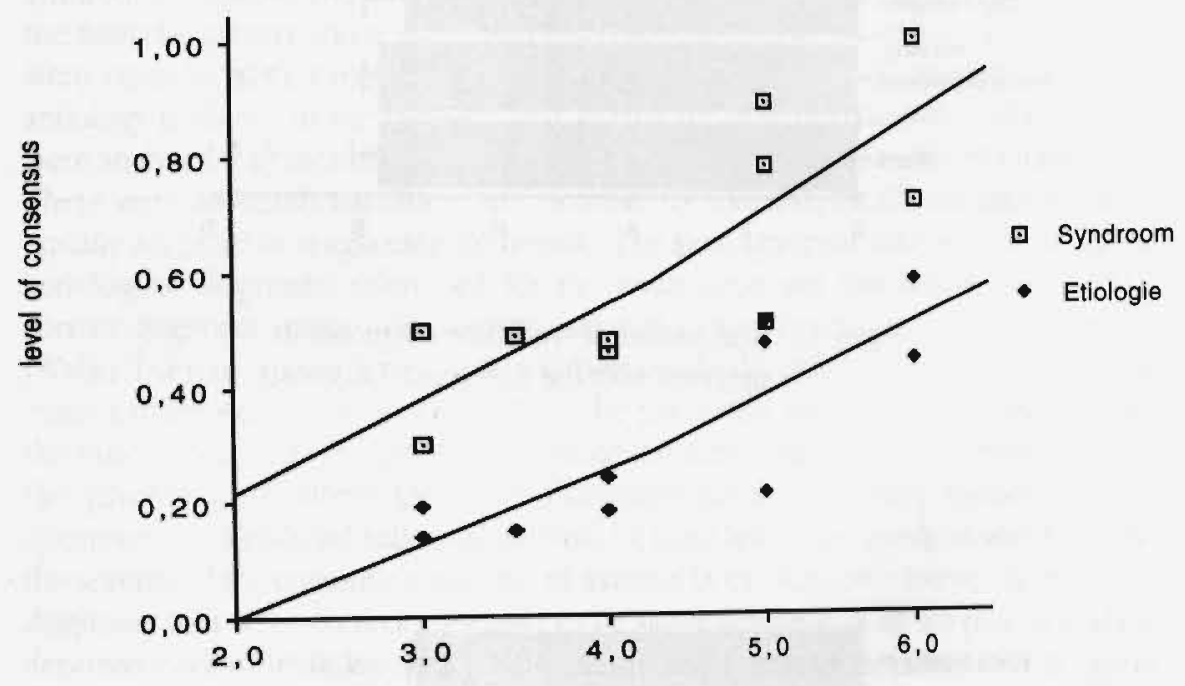

GDS score.

Figure 3.3 Relation between the level of consensus and the GDS score, for syndrome and aetiology.

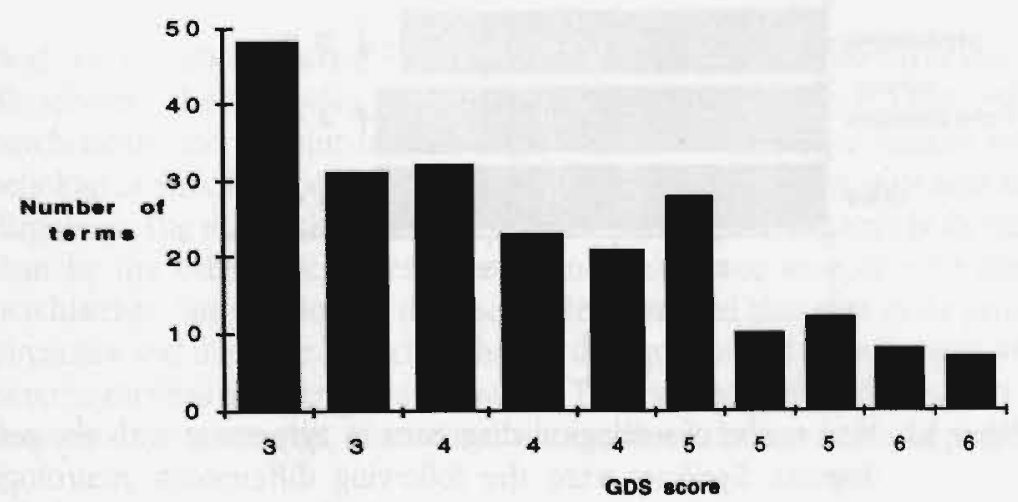

Figure 3.4 Number of terms used by 85 participants of the $\mathrm{CBO}$ consensus meeting on dementia for the cognitive syndrome in ten factious cases 
In patients with a mild or moderate degree of cognitive decline, there was a greater diversity of diagnostic opinions than compared with the more severely disturbed patients. This was the case for both syndromal and aetiological diagnoses ( $R=0.66$ and 0.70 , respectively, $\mathrm{p}<0.05$ ). Thus, almost every participant diagnosed dementia in patients with severe dementia.

The number of different terms that were used to designate the proper diagnosis by the GDS score score of each patient are presented in figure 4. As can be seen, the number of terms increased dramatically in the lower GDS stages.

\section{Discussion}

Several findings have emerged from this study. In the first place, we could not establish any change in the level of consensus one week after the consensus meeting, compared to the situation before the meeting. This finding can be explained in various ways. It is possible that the respondents had already reached a high level of consensus on the diagnosis of dementia before the meeting took place. The syllabus that was sent to all participants may have contributed to this unanimity. It is also possible that those participants who responded to the inquiry formed a selected group of people with a more than average interest in and knowledge of dementia. A third possibility is that the information from the oral presentations and the discussion at the meeting was too limited and fragmentary. However it seems more probable that the time between the first (pre-meeting) and the second (post-meeting) inquiry was too short to achieve any substantial change: physicians who have established their own way of diagnosing dementia over many years may not change their use of criteria and terms within two weeks, even though their diagnostic procedure does not correspond to that proposed at the consensus meeting. A third inquiry after a longer period of time would be necessary in order to assess the effects of the consensus meeting with more confidence.

Second, the level of consensus correlated directly with the severity of the cognitive decline. At first sight, this may seem a quite obvious finding, since the diagnosis of mild dementia is generally regarded as more difficult than that of a more advanced stage. However, upon further consideration this finding is remarkable, because the problem of mild dementia is especially formed by the fact that the condition is insufficiently recognised [124]. "Mild dementia is a latent condition, detectable only by challenge [309]". In our study, all the necessary elements were presented beforehand. Thus, the failure to recognize symptoms of dementia was precluded as a possible source of diagnostic error. Thus, a more probable explanation may be that the diagnostic criteria were applied less vigorously in patients with mild cognitive decline than in patients with more severe dementia. It is also possible that clinicians are more reluctant to use the stigmatizing term of dementia for patients with milder forms of cognitive decline, although it is realized that the regular diagnostic criteria (such as those of DSM-III-R [7]) are met. Both situations may lead to a semantic confusion between clinicians, since the functional significance of the term is disregarded [315].

Third, the number of correct diagnoses, i.e. the agreement with the Reference Diagnosis, was considerably influenced by the presence of depressive symptomatology. Even the 
Multidisciplinary Committee that served as the 'gold standard' could not reach full consensus on patients 4 and 6 , in whom there was a combination of dementia and depression. Apparently, standard criteria are more difficult to apply to patients with a combined symptomatology.

Fourth, the results of this study clearly show that the various disciplines focus upon different aspects of the dementia syndrome and its multidimensional aspects. For example, neurologists and psychiatrists, not so long ago still united in the specialty of neuropsychiatry, now seem to be each other's antipoles. This may be ascribed to differences in diagnostic traditions between the disciplines: neurologists are generally trained in diagnosing nosological entities, whilst psychiatrists are more inclined to use a descriptive and functional diagnostic approach [188]. Both ways of diagnosing complement each other.

The level of agreement with the RD reached for the syndromal diagnosis was on average higher than for the aetiological diagnosis. However, it should be noted that there were more diagnostic possibilities to chose from with regard to the aetiological diagnoses. The relatively low agreement with the RD of the psychiatrists and, to a lesser extent, the nursing home physicians, was marked significant. Apparently, these disciplines are used to a mere syndromal approach.

Some methodological issues of the present study have to be considered. The use of the diagnostic outcome of the Multidisciplinary Expert Committee (MEC) as the 'gold standard' can be debated. The diagnoses made by the MEC could not be compared with post-mortem data. However, the diagnosis of dementia is characterized by the absence of a clear, objective, and unambiguous diagnostic marker. Dementia is a clinical syndrome that is entirely defined by consensus [207]. The same is true for the most frequent causes of dementia, notably Alzheimer's disease, if neuropathological data are not available. In the absence of such external criteria, there is no other standard than the careful application of generally accepted diagnostic criteria. Thus, the diagnoses can be made most reliably by inviting a number of experts from relevant fields and asking them to adhere to the consensus criteria, after ample discussion of each case.

The question could be raised whether the findings of our study, in which written case reports were used, can be generalized to daily practice with 'real' patients. To avoid this source of error, the present investigation should have been carried out with living patients or with videotaped interviews, but such a design would have caused many practical problems. Reduction of the realistic situation by written case reports may have influenced the results of this study in a negative way. However, many of the respondents are faniliar with written case reports, although it is not known whether this is the same for every discipline. Assessment of a patient by reviewing their files ('paper visits') is not unusual in daily clinical practice. Therefore, we do not expect the manner on which the clinical data were presented would have had a substantial effect on our results. It can be stated that the amount of clinical data presented in the case reports of our study is probably much larger, and from more different disciplines, than is usually the case in daily practice.

The extrapolation of the findings of our study to all members of a particular discipline should also be given due consideration. Although 85 (67\%) out of 127 clinicians responded to 
both the first and the second inquiry, they account for only $18 \%$ of the total number of participants. It can be questioned whether those who attended the consensus meeting and, more specifically, those who responded to the inquiry, can be regarded as representative for their profession. As is the case in all inquiries in which the response is voluntary, the respondents cannot be regarded as a random sample. To quote Rumke: "Regarding the apparent objectivity of a statistical conclusion, it should be noted that the decision that the sample can be viewed as random is often subjective itself'. It is clear that the respondents of our study do not represent the average clinician: they probably had more affinity for psychogeriatry than randomly selected clinicians. An indication for this is the mean duration of the respondents' clinical experience, which is more than 10 years (Table 2). Therefore, the level of consensus and the agreement with the Reference Diagnosis will probably be lower in less selected samples of diagnosticians.

\section{Conclusion}

A systematic and multidisciplinary approach, based on generally accepted and validated criteria, has frequently been recommended for the diagnosis of dementia $[7,53,54,167,207$, 281,322 . The present study illustrates the need of such an approach. The study demonstrates that different disciplines focus upon different aspects of the multidimensional problem of dementia. Together, the disciplines form the complete diagnosis. Especially for patients with mild stages of cognitive decline and with a combination of cognitive and depressive symptomatology, a more vigorous adherence to diagnostic criteria is required. In these patients there is much diversity of diagnostic opinions. This aspect should be given more emphasis in the continued education of every clinician who is involved with dementia. 



\section{4}

\section{Diagnosing dementia: \\ a comparison between a monodisciplinary and a multidisciplinary approach*}

\section{Introduction}

In the last decade, a consensus has been reached on the diagnostic criteria for dementia and Alzheimer's disease $(\mathrm{AD})[7,207]$. The National Institute of Neurological Diseases and Communicative Disorders and Stroke and the Alzheimer's Disease and Related Disorders Association (NINCDS-ADRDA) workgroup proposes a broad multidisciplinary and systematic approach for the diagnosis of $\mathrm{AD}$. This includes extensive history taking from the patient and a knowledgeable collateral source as well as physical, neurological, psychiatric and neuropsychological examination. The workgroup recommends documentation by validated scales such as Folstein's Mini-Mental State Examination (MMSE) [97], the Ischemic Score [119], Hamilton's Depression Rating Scale (HDRS) [120] and the Blessed Dementia Scale (BDS) [24]. Moreover, laboratory assessments and computed tomography of the brain are required to diagnose secondary causes of dementia. Although originally designed for research purposes, the NINCDS-ADRDA criteria have been widely accepted as the basis for diagnostic procedures in clinical practice as well $\{40,54,66\}$.

The validity and reliability of the DSM-III and NINCDS-ADRDA criteria has been investigated extensively and appear to be highly satisfactory $[26,175,194,215,261,310]$. It was concluded from these studies that careful use of such a standard classification system, with standard assessment techniques, is important to minimize the variance between clinicians [194].

\footnotetext{
* FRJ Verhey, J Jolles, RWHM Ponds, N Rozendaal, LA Plugge, HCW de Ver, FW Vreeling and PJM van der Lugt. Joumal of Neuropsychiatry and Clinical Neurosciences, 1993; 5; 78-85
} 
Although these criteria have been widely used since their introduction, the way these NINCDS-ADRDA and other criteria are applied in routine clinical practice has received little attention. A multidisciplinary approach to dementia is generally recommended $[179,253,290$, 308 ], but in daily practice the diagnosis is usually made by a member of a single discipline, who lacks the expertise of other relevant disciplines. Given the multiconditional nature of dementia, discrepancies can be expected between the diagnostic outcome from a monodisciplinary and a multidisciplinary approach, but exact figures on this topic are, to our knowledge, sparse.

In a previous study $[244,317]$, we compared the diagnoses of ten case descriptions, made by 85 participants in a Dutch consensus meeting on dementia from varying specialties with those of a multidisciplinary expert committee, regarded as the "gold" standard. We found that 1) neurologists reached a significantly higher agreement with the committees etiological diagnoses than the psychiatrists did; 2) all specialties were comparable with regard to the syndromal diagnosis, i.e. whether dementia was present or not; and 3) depression was diagnosed more often by psychiatrists than by neurologists. However, this inquiry concerned written case reports in which all data relevant for the diagnosis dementia and its etiology had been presented beforehand. The major difficulty in practice is to recognize and to decide about the presence or absence of clinical symptoms; thus, this study did not provide insight into the way diagnoses are made in the less structured situation of daily practice.

The aim of the present study was to obtain insight into possible differences in diagnostic outcome between a systematic multidisciplinary approach and a monodisciplinary one in common clinical practice, particularly with respect to the diagnoses of dementia, Alzheimer's disease, and depression. We therefore compared the diagnoses made by the Maastricht Memory Clinic (MMC), which uses a standardized multidisciplinary approach [322], with the original referrer's diagnosis.

\section{Methods}

Four hundred and thirty consecutive patients, referred between May 1986 and July 1991 to the outpatient MMC of the University Hospital of Maastricht were evaluated. All patients had been referred because of dementia or memory complaints associated with aging. The patients were referred by general practitioners $(n=151)$; neurologists $(n=175)$; psychiatrists or the local psychogeriatric service $(n=77)$; or others, mainly internists or geriatricians $(n=27)$.

\section{Referrer's diagnosis:}

Before a case was evaluated by the $\mathrm{MMC}$, the referrer's diagnosis was obtained from a standardized admission form or from the referrer's letter. Referrers were not made aware of the present study since the aim of the study was to investigate the diagnostic outcome in common daily practice; announcing the purposes of the study would have influenced the diagnostic behavior of the referring clinicians. The MMC assessment was made within 4 weeks 
of the first referrer's assessment. The comparison of diagnostic outcomes pertains only to the cognitive, emotional or behavioral syndrome and the related neuropsychiatric diseases.

MMC diagnosis:

All patients received an extensive diagnostic assessment, which lasted about 4 to 5 hours. The diagnostic program is summarized in table 1 . The history and physical, neurological, psychiatric and neuropsychological examinations were always performed again by the MMC for each patient. Ancillary tests were not routinely repeated if they had been done previously within a reasonable period of time. The criteria for dementia and other psychiatric conditions were those of DSM III and DSM-III-R $[6,7]$. The diagnosis of probable or possible Alzheimer's Disease (AD) was made according to the NINCDS-ADRDA criteria [207]. An Ischemic Score (IS) [119] of less than 5 was also required. An IS of 5 or more was seen as indicative of a cerebrovascular etiology, in the absence of other causes. Other neurological and somatic conditions were diagnosed according to usual clinical guidelines. The neuropsychological investigation has been described extensively elsewhere [146] and has been devised to assess all relevant cognitive functions in the domain of memory, attention, language, praxis, perceptual functions, and speed of information processing. Briefly, a battery of standard psychometric tasks was combined with a neurobehavioral examination according to LuriaChristensen [42,195] and information processing tasks [297]. The standard tests were: Rey auditory verbal learning task, Trail Making Test, Stroop test, symbol- digit modality test, digit span, fluency, various subtests of the Wechler Adult Intelligence Scale (WAIS), and tests for complex visual functions. After the examinations had been completed, the results were discussed at a weekly interdisciplinary meeting. After discussion in this forum, a definite syndromal and etiological diagnosis was made, and a proposal for treatment was formulated. Afterwards, a letter was sent to the referrer in which all diagnostic elements, final diagnosis and proposal for treatment were described.

\section{Analysis of the data}

A two-step procedure was used to classify the diagnoses of the referrer as well as of the MMC. First, it was decided whether a syndrome of dementia was present or not and whether cognitive disorders were objectified or not. This was done because the diagnostic criteria of many neuropsychiatric diseases require the presence of dementia $[7,207]$. For example, for the diagnosis of Alzheimer's disease, the presence of dementia is a prerequisite.

The next step was to classify the etiology of the syndrome, with the following alternatives: 1) Not specified (NS), 2) Probable or possible Alzheimer's disease (AD), 3) cerebrovascular cause, e.g., multi-infarct dementia and focal ischemia (CV), 4) Neurological cause other than cerebrovascular, e.g., Parkinson's disease and head trauma (NE), 5) Somatic/ internal cause, e.g, metabolic disorders (SO), 6) Intoxication/ side effect of drugs, e.g., major tranquilizers (IN), 7) Mood disorder, e.g. major depression and dysthymia (DP)8) Psychiatric or psychological disorder other than 7, e.g. psychosis or personality disorder (PS). 
When there was more than one possible cause, the patient was classified according to the most probable cause for the cognitive deterioration. For this analysis, depression was seen as a cause for the cognitive syndrome and not as a syndrome by itself. The diagnoses of the memory clinic served as a reference for the calculation of the specificity and sensitivity of the referrer's diagnoses.

Table 1. Standard diagnostic procedure in Maastricht Memory Clinic

\section{Neuropsychiatry:}

History from patient and significant other

Medical history

Life events, premorid functioning

Physical examination

Neurological examination

Psychiatric examination

Blood tests (hematology, glucose, biochemical analyses, TPHA, vitamins, TSH)

ECG, chest X-ray

EEG when epilepsia is suspected

CT-scan when cognitive decline is objectified

Neuropsycbology [146]:

Psychometric tasks

Luria-Christensen neuropsychological investigation

Information processing tasks

\section{Rating scales:}

Mini Mental State Examination

Hachinski's Ischemic Score

Hamilton Depression Rating Scale

Blessed Dementia Scale

Global Deterioration Scale

(for degree of cognitive dysunctions)

(for documentation of cerebrovascular factors)

(for degree of depressive symptoms)

(for degree of behavioural dystunctions)

(for global assessment of cognitive dedine)

\section{Results}

Of the 430 patients evaluated, 111 patients had been referred because of dementia; its presumed etiology was specified in 74 cases. The remaining 319 had been referred because of memory complaints without any reference to the diagnosis of dementia. In this group, the referrer specified the presumed etiology in 189 cases. Table 2 lists the patient characteristics and MMC findings by the different referring disciplines. Sixty-ive percent of the patients were between the age of 45 and 75 years. Patients referred by the psychiatrists were younger, whereas those referred by the general practitioners and 'other referrers' were somewhat older than the average of 61.7 years. 
Table 2. Distribution of patient characteristics of 430 memory clinic patients by specialty

\begin{tabular}{|c|c|c|c|c|c|c|}
\hline \multirow{2}{*}{$\begin{array}{l}\text { Characteristics from } \\
\text { MMC evaluation }\end{array}$} & \multicolumn{2}{|l|}{ Coneml } & \multicolumn{3}{|c|}{ Patients referred by } & \multirow[b]{2}{*}{$P<*$} \\
\hline & Practitioner & Neurologist & Psychiatrist & Others & Tonal & \\
\hline N & 151 & 175 & 77 & 27 & 430 & \\
\hline mean age (yrs.) & 64.9 & 60.1 & 57.9 & 64.8 & 61.7 & 0.001 \\
\hline education a & 4.1 & 4.0 & 4.1 & 4.6 & 4.1 & n.s. \\
\hline $\begin{array}{l}\operatorname{sex}(\mathrm{MN}) \\
\text { Syndromal diagnasis, } N(\%)\end{array}$ & $78 / 73$ & $100 / 75$ & $49 / 28$ & $15 / 12$ & $242 / 188$ & \\
\hline dementia & $58(38 \%)$ & $55(31 \%)$ & $24(31 \%)$ & $15(56 \%)$ & $152(35 \%)$ & \\
\hline cognitive disorder & $55(36 \%)$ & $103(59 \%)$ & $42(55 \%)$ & $9(33 \%)$ & $209(49 \%)$ & \\
\hline no cognitive disorder & $38(25 \%)$ & $17(10 \%)$ & $11(14 \%)$ & $3(11 \%)$ & $69(16 \%)$ & \\
\hline Scores, mean & & & & & & \\
\hline MMSE b & 23.1 & 26.1 & 23 & 20.1 & 24.1 & 0.005 \\
\hline GDSe & 3.1 & 3.2 & 3.2 & 4.2 & 3.3 & 0.005 \\
\hline BDS-Id & 4.1 & 2.9 & 4.3 & 7.0 & 3.8 & 0.005 \\
\hline HRSD e & 10.5 & 8.7 & 12.4 & 10.1 & 10.1 & 0.005 \\
\hline
\end{tabular}

\footnotetext{
* Kruskall-Wallis test

a education according to Verhage, $1.7 \mathrm{scale}$ : 1 is incomplete primary school, 7 is university [314]

b Score on the Mini-Mental State Exam of Folstein et al. [97]

c Score on the Global Deterioration Scale [255]

d Score on the Blessed Dementa Scale, first part (behavior, personality) [24]

e Score on the Hamilton's Depression Rating Scale, 17 items [120]
}

Cognitive and social functioning, as measured by MMSE and BDS, was on average better in the patients referred by the neurologists and worse in those referred by 'other referrers'. There were no patients with very severe dementia (i.e., score of 7 ) on the Global Deterioration Scale (GDS)[255]. In 69 patients (16\%), the complaints about cognitive functions could not be objectified by neuropsychological tests.

The sensitivity and specificity rates are shown in Table 3 for the referrer's diagnosis of dementia irrespective of its etiology; for dementia caused by $A D$; and for cognitive disorders without dementia, caused by depression or dysthymia. Because in daily practice the term 'dementia' with etiology unspecified is often used for Alzheimer's disease, rates were also calculated for primary degenerative and unspecified dementia together. In addition, sensitivity and specificity rates were calculated for depression together with other psychiatric conditions in order to examine whether refrrer and MMC agreed in the differentiation between organic or functional etiologic categories. 
Table 3. Sensitivity and specificity of referrer's diagnosis of dementia, Alzheimer's disease and depression, by specialty.

Patients referred by

Discipline

General Neurologist Psychiatrist Others Total

Practitioner

$(n=151) \quad(n=175) \quad(n=77) \quad(n=27) \quad(n=430)$

Dementia syndrome

$\begin{array}{llllll}\text { prevalence } & 0.38 & 0.31 & 0.31 & 0.56 & 0.35 \\ \text { sensitivity } & 0.60 & 0.73 & 0.38 & 0.53 & 0.61 \\ \begin{array}{l}\text { specificity } \\ \text { zheimer's disease }\end{array} & 0.99 & 0.89 & 0.94 & 0.83 & 0.93 \\ \text { prevalence } & & & & & \\ \text { sensitivity } & 0.25 & 0.15 & 0.22 & 0.22 & 0.20 \\ \text { specificity } & 0.18 & 0.33 & 0.12 & 0.33 & 0.23 \\ & 0.98 & 0.95 & 0.97 & 0.95 & 0.96\end{array}$

Alzheimer's disease

(including referrer's diagnoses of "unspecified dementia")

$\begin{array}{llllll}\text { prevalence } & 0.25 & 0.15 & 0.22 & 0.22 & 0.20 \\ \text { sensitivity } & 0.55 & 0.63 & 0.24 & 0.50 & 0.51 \\ \text { specificity } & 0.96 & 0.92 & 0.93 & 0.81 & 0.93 \\ \text { Depression } & & & & & \\ \text { prevalence } & 0.22 & 0.16 & 0.36 & 0.07 & 0.21 \\ \text { sensitivity } & 0.45 & 0.21 & 0.68 & 0.00 & 0.44 \\ \text { specificity } & 0.97 & 1.0 & 0.96 & 1.00 & 0.99\end{array}$

Depression and other psychiatric conditions

$\begin{array}{llllll}\text { prevalence } & 0.33 & 0.25 & 0.44 & 0.15 & 0.31 \\ \text { sensitivity } & 0.42 & 0.16 & 0.62 & 0.00 & 0.37 \\ \text { specificity } & 0.92 & 0.99 & 0.88 & 1.00 & 0.95\end{array}$

Of the 279 patients not fulfilling the criteria of dementia, only 19 (7\%) had been referred as being demented. In 16 of these 19 patients, the DSM-III $(-R)$ criterion that cognitive disturbances must lead to a significant interference with daily activities was not fulfilled. Depressive symptoms coexisted with a neurological disorder, most often cerebral infarction and Parkinson's disease, in 11 of these 19 patients. On the other hand, in 60 of the 152 demented patients ( $39 \%$ ), a diagnosis of dementia had not been made previously. Underreporting of dementia occurred especially with mild dementia: the sensitivity rates for the referrer's diagnosis of dementia were for the GDS stages $3,4,5$ and $6: 0.50,0.54,0.64$ and 0.79 , respectively. The presence of depressive symptoms was another factor that influenced the diagnosis dementia: sensitivity rates for patients with a HRSD score of $<13$ were 0.68 , whereas for patients with a HRSD $\geq 13$ these rates were only 0.36 . The sensitivity rates were 
the lowest for the psychiatrists' diagnoses of dementia and the highest for those of the neurologists; the general practitioners and the other referrers were in between. The same pattern of sensitivity was seen for the diagnosis primary degenerative dementia, whether clustered or not with unspecified dementia. The opposite of this pattern, however, could be seen for the sensitivity rates for depression, with or without the inclusion of other psychiatric conditions. Fifty of the 89 patients with depression without dementia and 82 of the 130 patients with nondementing psychiatric conditions had not been diagnosed previously as such. Neurologists tended to underreport these conditions.

Table 4. Agreements and discrepancies in diagnostic classifications between referrer and Memory Clinic $(\mathrm{N}=4,30)$

Diagnosis by referrer

Diagnosis by Memory Clinic

Dementia

No Dementia

Total by

rejerrer

Dementia

code NS AD CV NE SO IN DP PS

NS AD CV NE SO IN DP PS

Not specified

NS

2562

- $11 \cdot 1 \cdot 137$

Primary degenerative AD

2063

Cerebro vascular

$\mathrm{CV}$

-

2 .

Other neurologic cause NE

Somatic/internal cause SO

Intoxic/drug side effect IN

Depression

DP

Other psychiat disorder PS

\section{No dementia}

Not specified

code NS AD CV NE SO IN DP PS

Primary degenerative $\mathrm{AD}$

Cerebrovascular

Other neurologic cause NE

Somatic/intemal cause SO

Intox. drug side effect IN

Depression

DP

Other psychiat disorder PS

Total diagnosed by $M C$

NS AD CV NE SO IN DP PS

$\begin{array}{llllllll}24 & 4 & 4 & -3 & 30 & 24 & 130\end{array}$

$-6-5 .-11$

- $63-\quad 7278$

- - $\quad-816429$

- - 5118

$111 \cdot .39 \cdot 45$

- $\quad 11.3918$

Note: Values shown are numbers of patients. Figures printed in bold represent agreements in syndromal and etiological diagnoses between referter and Memory Clinic (MC) 
Table 5. Newly reported disorders in 430 patients from the Maastricht Memory Clinic:

\begin{tabular}{|c|c|c|c|c|c|}
\hline Patients referred by & $\begin{array}{c}\text { G.P. } \\
(n=151)\end{array}$ & $\begin{array}{l}\text { Neurologist } \\
(\mathrm{n}=175)\end{array}$ & $\begin{array}{l}\text { Psychiatrist } \\
(n=77)\end{array}$ & $\begin{array}{l}\text { Others } \\
(n=27)\end{array}$ & $\begin{array}{c}\text { Total } \\
(n=430)\end{array}$ \\
\hline \multicolumn{6}{|l|}{ Demented } \\
\hline CVA & 2 & 9 & 2 & - & 13 \\
\hline Vitamin deficiency & 2 & - & 2 & . & 4 \\
\hline Alcohol abuse & 1 & 1 & 1 & - & 3 \\
\hline Drug side effects & 1 & 1 & 1 & . & 3 \\
\hline Thyroid disorder & - & 3 & . & . & 3 \\
\hline Parkinson's disease & 1 & 1 & . & . & 2 \\
\hline Epilepsia & 1 & 1 & . & . & 2 \\
\hline ALS & 1 & & & & \\
\hline Depression / dysthymia & 9 & 7 & . & 3 & 19 \\
\hline Anxiety & 2 & 3 & 2 & - & 7 \\
\hline Psychosis & - & 1 & - & . & 1 \\
\hline Phobia/OCD & 1 & . & . & - & 1 \\
\hline \multicolumn{6}{|l|}{ Nondemented } \\
\hline Alcohol abuse & - & 6 & . & - & 6 \\
\hline CVA & 4 & 1 & 1 & - & 6 \\
\hline Drug side effects & - & 2 & 3 & . & 5 \\
\hline Cerebral neoplasm & 1 & 1 & . & . & 2 \\
\hline Parkinson's disease & . & 1 & . & 1 & 2 \\
\hline Vitamin deficiency & 1 & - & 1 & . & 2 \\
\hline Cerebral contusion & - & - & 1 & . & 1 \\
\hline ALS & - & . & 1 & . & $i$ \\
\hline NPH & - & - & 1 & . & 1 \\
\hline Epilepsia & 1 & - & . & . & 1 \\
\hline Sjögren disease & - & 1 & . & . & 1 \\
\hline Depression / dysthymia & 11 & 33 & 5 & 1 & 50 \\
\hline Adjustment disorder & 1 & 7 & . & . & 8 \\
\hline Personality disorder & 2 & 3 & 2 & - & 7 \\
\hline Phobia/OCD & - & 2 & - & - & 2 \\
\hline Dyslexia & 1 & - & - & . & 1 \\
\hline \multicolumn{6}{|c|}{ Subtotals of newly reported conditions ( $n$ ) } \\
\hline Somatic & 15 & 29 & 14 & 1 & 59 \\
\hline Psychiatric & 27 & 56 & 9 & 4 & 96 \\
\hline
\end{tabular}

Subtotals of newly reported conditions ( $n$ )

Somatic

Psychiatric

15

27

Ratio newly reported to knoun diagnoses (\%)

Somatic

Psychiatric
29

56
14

9

20

72

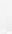

14

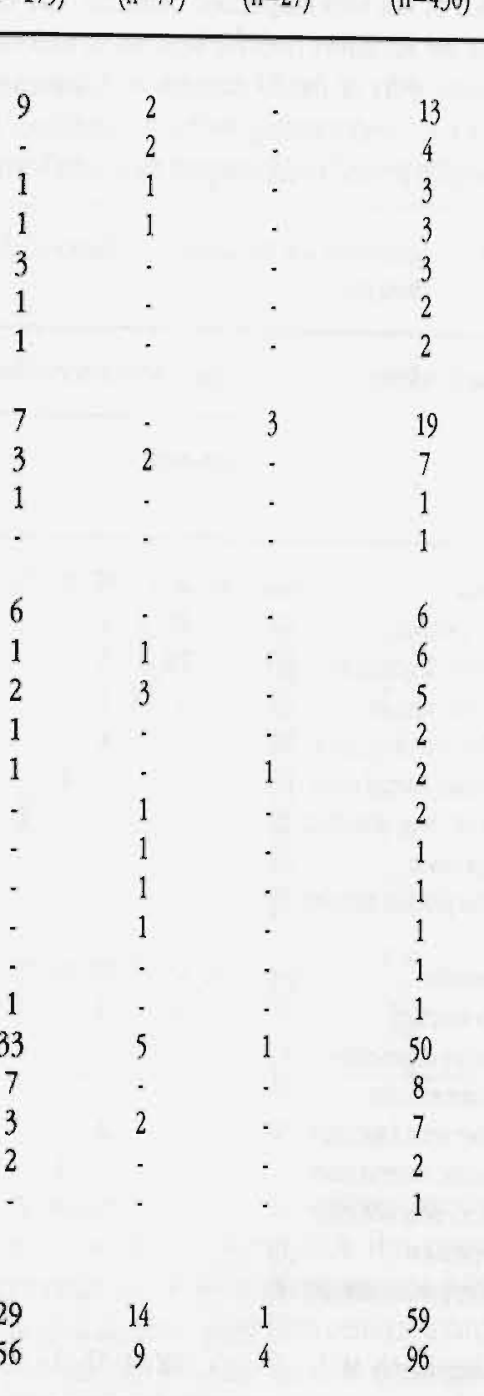


In Table 4, the agreements and discrepancies of diagnoses are shown in more detail for all patients. The overall accuracy rate for all referrers was $46 \%$; that is, in only 198 of the 430 patients did the referrer's and MMC's diagnostic classifications agree. When, more liberally, all unspecified-diagnosis patients not referred as demented were ignored, and when unspecified dementia was classified as primary degenerative, these diagnoses agreed in 199 of the 300 patients (66\%). Calculated for each discipline separately, these more liberal accuracy rates were as follows: general practitioners, $71 \%$; neurologists, $64 \%$; psychiatrists, $68 \%$; other referrers, $50 \%$.

In contrast to the above-mentioned classifications, which were limited to the most important cause, Table 5 enumerates all relevant diagnoses, other than dementia or Alzheimer's disease, that had not been reported previously by the referrer. In this analysis, depression and dementia were not perceived as mutually exclusive. If, for example, a demented patient also fulfilled the DSM-III-R criteria for depression (with the exeption of the last, nonorganic, criterion), both dementia and depression were rated. The findings were ranked according to cognitive status (demented or not) and to diagnostic category, i.e., 'psychiatric' (DSM-III-R's axis 1 and 2) or 'somatic' (axis 3).

As can be seen, $19 \%$ of the somatic diagnoses and $44 \%$ of the psychiatric diagnoses had not been reported previously. Neurologists and psychiatrists were, again, each other's counterparts: the majority of psychiatric diagnoses ( 56 out of $78,72 \%$ ) had not been reported earlier by the neurologists, whereas almost the half of the somatic diagnoses (48\%) had not been mentioned previously by the psychiatrists.

\section{Discussion}

The results of this study show that the multidisciplinary and systematic approach based on the NINCDS-ADRDA and DSM-II-R criteria leads to substantial differences in the diagnostic outcome compared to the monodisciplinary procedure usually adhered to in everyday practice. Disagreement between referrer's and the MMC's diagnoses for dementia and Alzheimer's disease occurred especially in mild dementia, in the presence of depressive symptomatology and in patients referred by psychiatrists. Somatic conditions with relevance for cognitive functioning were often unreported in the group referred by psychiatrists. On the other hand, depression and other psychiatric conditions went frequently unreported, most often in patients referred by neurologists. It should be noted, however, that the patient groups from the various referrers differed with respect to age and the scores on the MMSE, HDRS and BDS, and thus comparisons should be made cautiously. The findings suggest that neurologists and psychiatrists are complementary to each other, and emphasize the need of a combined approach to these multidimensional problems.

Before we discuss the possible implications of this study, a few methodological points have to be addressed. The validity of the MMC diagnoses can be debated. Validation of some of the etiological categories can be achieved by neuropathologic data, which were not available in this study. furthermore, the diagnosis of degenerative or vascular dementia can be validated by 
continued cognitive deterioration $[124,270]$. In another study we followed 110 patients with dementia, and it appeared that $95 \%$ of the patients had deteriorated in cognitive oand/ or social functioning after a mean follow-up period of 41 months, indicating the validity of the MMC diagnosis (Verhey et al., 1992, unpublished data: see chapter 8). Some diagnoses, such as dementia $[7,207]$, depression or other psychiatric conditions [7], are based exclusively on clinical data. Ideally, one would like to have a third set of diagnoses of all patients, independent of those of the MMC or referrer in order to compare the latter two approaches, but such a design would raise many practical problems.

In the absence of clear data on the validity of the MMC diagnoses, the use of sensitivity and specificity measures could be discussed. This study should not be seen as a comparison between 'true' or 'false'. We used sensitivity and specificity rates in order to obtain a quantitative measure for the differences between the diagnostic outcomes from a monodisciplinary, not standardized and subjective approach and a multidisciplinary, systematic and approach, based on clear criteria. However, it is reasonable to assume that the validity of the latter approach will be greater, and thus, with some precautions, can serve as a 'gold standard'. This approach, establishing a so-called 'authoritative opinion' [158], has been used in other studies as well $[108,197]$.

The fact that the refferer's diagnosis was noted by a MMC staff member and thus not obtained blindly is another possible methodological flaw of this study. However, this source of error was minimized by establishing the referrer's diagnosis before any part of the MMC evaluation started.

Finally, the fact that a patient was referred to the MMC might have influenced the accuracy of some referrer's diagnoses, both in a positive and negative way. It is conceivable that referrers were not as thorough in their diagnostic procedures as they would have been had no reference been available. In some cases the referrer's diagnosis may have been only preliminary. However, the opposite might also be the case, that is that feedback by way of MMC reports about earlier referred patients might have increased the chance of agreement on future diagnoses. It is not clear to what extent these factors might have influenced the results of this study.

Our findings confirm the results of previous investigations [194, 243, 244]. Comparisons between the diagnostic outcomes of referrers and a multidisciplinary team have been carried out previously $[108,128,179,197,329]$. In two studies [108, 128], the reference pattern and patient characteristics were comparable with those of the present study. Garcia et al. (1981) found that dementia was overdiagnosed in at least $26 \%$ of the patients, which is much higher than the $7 \%$ of our study. However, it is important to recognize that in Garcia's study all patients had been referred for suspected dementia, which automatically leads to overdiagnosis. In the other study [128], the overall percentage agreement between the tentative diagnosis at referral and the final diagnosis was $59 \%$, which approximates the percentages of $46 \%$ to $66 \%$ found in our study. In Garcia's study three errors were found to be important for the overdiagnosis of dementia: equating atrophy on CT-scans with clinical dementia, taking focal neuropsychological deficits for global, and failure to recognize depression. Of these, only the last was a common source of diagnostic discrepancy in our study. 
Another frequent factor for both over- and underdiagnosing dementia in our study was the failure to obtain information about daily functioning from a knowledgeable collateral source, a prerequisite needed to fulfill criterion C of DSM-III-R for dementia. This finding pleads for a more vigorous application of standardized scales that measure social functioning, such as the $B D S$, in routine diagnostic procedures.

Underdiagnosis often occurred in mildly demented patients and in patients with coexisting psychiatric symptoms or a specific neurological disorder. As has been stated by others [194], these factors may lead to the use of ambiguous terminology and to misinterpretation of the diagnostic criteria. Underreporting of dementia might be related to a reluctancy to use a stigmatizing diagnosis or to the fact that in common linguistic use the term 'dementia' is reserved for Alzheimer's disease. In both situations, however, the avoidance of the term dementia is inappropriate, since it disregards the functional significance of the diagnosis of dementia; namely, that the patient is, at least to a certain degree, dependent on his or her environment for extra support and care.

\section{Conclusion}

This study suggests that there is a need for a standardised and multidisciplinary diagnostic model for the diagnosis of dementia and other age-associated cognitive disorders, using wellaccepted and clear, explicit terms. In the last decade the emphasis on the diagnosis of dementia has shifted from the search for a single reversible cause to the detection of all somatic and psychiatric conditions that may influence cognitive functioning $[13,179]$. This study shows that many chances to influence these conditions go unrecognized without a broad multidisciplinary approach. Although this study does not allow a strict comparison between the specialties, the results suggest that neurologists and psychiatrists complement each other in diagnosing dementia and depression. This underlines the advisability of more frequent use of an integrated neuropsychiatric approach for cognitively disturbed patients. 



\section{The validity of the diagnosis of dementia: a follow up study of demented patients from the Maastricht Memory Clinic}

\section{Introduction}

One of the major steps forwards in research in geriatric psychiatry has been the development of criteria for the clinical diagnosis of dementia and dementing illnesses. At present, the criteria of DSM-III-R for the syndrome of dementia [7], and those of the work-group of the National Institute of Neurological and Communicative Disorders and Stroke (NINCDS / ADRDA) for Alzheimer's disease (AD) [207] are widely accepted. The diagnosis of mild or moderate dementia is notoriously more difficult than in more advanced stages because the history of symptoms is usually shorter and the clinical picture is not fully developed and shows more overlap with that of other mental conditions such as depression, focal neuropsychological deficits or normal age-associated cognitive impairment. In contrast to the situation in more severe stages, the prevalence of mild dementia varies widely depending on which set of criteria has been used. For instance, in a study comparing five current sets of criteria for mild dementia, the frequency varied from 3 to 64 per cent [219].

There are no simple bedside screening instruments with sufficient sensitivity for the mildest stages of dementia. This diagnosis requires clinical judgement and a certain level of experience, given the amount of data that has to be considered [229]. For instance, the DSM-III-R criteria appear to classify dementia successfully, ie, are highly predictable with regard to a progressive course, when adhered to by experienced clinicians [99]; however, when the same criteria were applied by untrained research assistants, the diagnosis of dementia had much less prognostic significance [232]. Thus, the clinical diagnosis of relatively mild dementia still carries some uncertainty and must be validated, even when it is made by using of generally accepted research criteria. Given the difficulties of obtaining in vivo 
neuropathological data, documentation of a progressive cognitive or behavioural decline offers an important alternative for validation of the diagnosis $\mathrm{AD}$ or $\mathrm{VD}[270]$. Athough a progressive course is not required for the diagnosis of dementia, it is characteristic of most of the dementing disorders $[7,46,207]$. As dementia progresses and cognitive or social deterioration becomes more evident, there is less uncertainty about the diagnosis, whereas the diagnosis can be questioned when there is no such decline.

The purpose of the present study was to examine the number of patients who showed progressive decline on follow-up in a sample of patients with $\mathrm{AD}$ and vascular dementia (VD) with mild or moderate dementia, as diagnosed by using current research criteria. These numbers give an indication of the accuracy of the clinical diagnoses and thus of the validity of the diagnostic method that was used. Because patients with aetiologies other than $\mathrm{AD}$ or VD, such as alcohol-related dementia or head trauma dementia, have a more unpredictable clinical course, only the former two aetiological categories were included in this study.

\section{Patients}

Consecutive outpatients from the Maastricht Memory Clinic (MMC) were examined. Patients were included when they 1) fulfilled the DSM-III-R criteria for dementia [7];2) fulfilled the criteria of probable $\mathrm{AD}$ [207] or VD, according to DSM-III-R criteria and a score of four.or more on Rosen's modified Ischemic Score [269], 3) had been investigated at least eighteen months before the study took place. This period was taken as a arbitrary minimum period of follow-up. The diagnostic procedure was based on recent research criteria $[7,207]$ and is described in more detail elsewhere [316]. The procedure included, in short, a semi-structured history, somatic, neurological, psychiatric investigations and an extensive neuropsychological examination; the modified Ischemic Index of Rosen et al. [269]; the Global Deterioration Scale, GDS [255]; the Blessed dementia scale, BDS [24]; Folstein's' Mini Mental State Examination, MMSE [97]; and Hamilton's depression rating scale, HRSD [120]. The syndromal and aetiological diagnoses were made by the author of this thesis after discussion of the clinical findings and the results of the ancillary examinations.

\section{Methods}

Available data that could provide insight into the course of the symptoms since the first assessment in the MMC were collected by a research assistant who did not know the patients. Sources of information encompassed notes in the patient records, including those from other regional departments such as the psychogeriatric service from the Institute of Social Psychiatry (RIAGG), and the minutes of the regional 'psychogeriatric platform'. In this meeting: representatives of the five local professional institutes that are concerned with psychogeriatric care discuss weekly arising problems with demented patients in the Maastricht region. Additionally, requests for admission to a nursing home are always discussed at this meeting, 
and extensive minutes are taken. If information could not be obtained from these sources, the general practitioner was contacted and asked to rate changes in general functioning, cognitive functioning and need for extra support on a five point scale (much better, slightly better, unchanged, slightly worse, much worse).

On the basis of these data, the research assistant judged whether a patient had deteriorated or not. In the cases that were not covered by the above-mentioned descriptions, the clinical course was supposed not to be progressive. Cognitive and social deterioration was defined as follows:

\section{Cognitive deterioration:}

decline as assessed by from quantitative data from cognitive tests, such as a $10 \%$ reduction or more of the score on the MMSE [97] or other neuropsychological tests, of written descriptions, from professionals other than those of the MMC, from which cognitive deterioration was apparent, such as obviously increased forgetfulness or aphasia, or evidence of progressive cognitive dedine as reported by the general practitioner.

\section{Functional deterioration:}

increased need of support in daily activities, apparent from intensive attendance by social psychiatric nurse, distric nurse or comparable organizations, other than the $\mathrm{MMC}_{8}$ or placed in a nursing home or on the waiting list for it in case no need for these measures existed at the date of the first assessment in the $\mathrm{MMC}$, or written descriptions, from professionals other than those from the $\mathrm{MMC}$, by which decreased behavioural functioning was apparent, such as wandering or overt aggressive outburst, or evidence of progressive functional dedine, as reported by the general practitioner.

\section{Results}

One hundred and fourteen patients, 81 with $\mathrm{AD}$ and 33 with VD were included in this study. The patients' main characteristics are shown in table 1 . Fifty-three patients had mild dementia (as defined by a GDS score of 3 or 4), 46 were moderately demented (GDS 5), and 15 were severely demented (GDS6). The average period of follow-up was 41 months (16.7-71.2).

\section{Source of information}

Four patients $(4 / 114,3.6 \%)$ could not be traced. Thirty-six patients had been described by more than one source and in none of these cases were there inconsistencies in the information. The sources of information were as follows: a) 59 patients had been followed in the MMC, b) 60 patients had been discussed in the psychogeriatric platform, c) 22 patients had 
been described in patient records from other institutes and d) 18 patients had been seen by their general practitioner.

Table 1. Patient characteristics

\section{Alzheimer's disease vascular dementia}

$\begin{array}{lll}\mathrm{N} & 81 & 33 \\ \mathrm{M} / \mathrm{F} & 34 / 47 & 21 / 12 \\ & & \\ \text { Age (mean, years.) } & 72.0 \pm 8.9 & 74.7 \pm 7.3 \\ \text { range } & 50-88 & 58-88 \\ & & \\ \text { GDS (mean, range) } & 4.5(3-6) & 5.0(4-6) \\ \text { MMSE (mean, range) } & 17.9(3-26) & 14.7(6-24) \\ \text { BDS (mean, range) } & 6.8 & 9.3(3-23)\end{array}$

\section{Evidence of progressive decline}

Information about cognitive decline was available for 104 patients. Of these, 99 patients ( 95 \%) had been described as having deteriorated cognitively, while in 6 patients the cognitive functions had not clearly changed ( 5 patients) or had improved (1 patient).

With regard to changes in social functioning, information could be obtained for all 110 patients. Twenty-four were still living in their own home without extra help, whereas 3 needed extra professional support. Fifty patients had moved into a psychogeriatric nursing home, 4 were on the waiting list for a nursing home and 13 patients could no longer live independently without professional support. Sixteen patients died since the MMC assessment; in 13 of these patients, cognitive and social functioning had deteriorated in the period before death. Information about the clinical course of the other 3 deceased patients was not available. All patients with social deterioration were reported to have also declined cognitively,

Taken together, 95\% $(99 / 104)$ of the patients traced showed evidence of progressive deterioration. Taking into account the patients who could not be traced or for whom there was too little data, this rate of decline varied between $87 \%$ (99/114, worst case) and $96 \%$ (110/114, best case).

Athough further statistical analysis was not meaningful due to the small numbers, there were no clear differences between patients with $\mathrm{AD}$ and $\mathrm{VD}$ (rates of decline 88.6 and $90.3 \%$, respectively). Slightly fewer patients with mild dementia declined cognitively, compared to those with moderate dementia and with severe dementia, as measured by the GDS score (GDS 3 and 4: 89\%, GDS 5: 97\%, GDS 6: 100\%). The characteristics of patients who did or did 
not show mental decline are shown in table 2. On average, the follow-up period for the nondecliners was 7 months shorter than for the decliners. No substantial differences could be found in age, educational level and the scores on Hamilton's depression scale. The nondecliners were slightly less cognitively disturbed, but had somewhat lower scores than the decliners on the Blessed dementia scale, which reflects social functioning.

Table 2. Comparison between patients who had or had not declined at follow-up

\begin{tabular}{lll}
\hline & Dedined & Not decdined \\
\hline $\mathrm{N}$ & 99 & 5 \\
M/F & $48 / 56$ & $4 / 1$ \\
Number ADND & $71 / 28$ & $3 / 2$ \\
& & \\
Age (mean, years) & $73.1 \pm 8.7$ & $71 \pm 3.4$ \\
Education(1-7) [314] & 3.6 & 3.7 \\
GDS score & $4.65 \pm 0.8$ & $4.2 \pm 0.4$ \\
MMSE score & $17.1 \pm 5.9$ & $19.8 \pm 4.2$ \\
BDS score & $7.7 \pm 4.2$ & $4.8 \pm 2.0$ \\
HRSD score & $8.9 \pm 4.9$ & $7.8 \pm 1.7$ \\
Follow-up period (months) & $41.4 \pm 16.6$ & $34.3 \pm 18.2$ \\
\hline
\end{tabular}

\section{Discussion}

The main purpose of this study was to establish the number of patients who showed a progressive decline, in order to validate the current diagnostic methods for the clinical diagnosis of dementia caused by Alzheimer's disease and vascular dementia. The data indicate that $95 \%$ (86-96\%) of the patients demonstrated the expected course of progressive deterioration, ie, only 6\% (4-14\%) may have been misdiagnosed. Given the large proportion of mild and moderate dementia in this population, this figure can be regarded as satisfactory and illustrates the validity of the clinical diagnostic methods used.

Previous studies reported higher rates of misclassification [158]. In one often cited follow-up study, the percentage of misclassification for dementia after four years was 31 per cent, which could be ascribed for the greatest part to misdiagnosed depression [267]. It is likely that the high misclassification rate found in this study was related to the lack of standardized diagnostic criteria at the time of the study. Later studies on this subject, in which diagnostic criteria were more explicitly applied, suggested lower frequencies of misdiagnosis 
on follow-up, ie, between 1 [180] and 18 [290] per cent, but the degree of dementia in the patients of these studies was on the average more severe.

Because of the qualitative and global nature of the follow-up data, the results of this study should be regarded with caution. It should be noted that the absence of cognitive decline does not rule out a progressive dementing disorder since plateaus in the clinical course may occur [207] or the follow-up period may have been too short. Thus, the real rate of progressive deterioration may be higher than that found in this study. However, evidence of a continuous progressive course does not necessarily imply a diagnosis of $\mathrm{AD}$ or $\mathrm{VD}$, as other degenerative brain diseases share this characteristic as well.

In summary, we conclude that the diagnosis of dementia by the DSM-III-R criteria [8], of Alzheimer's disease by the NINCDS-workgroup [207] and the criteria for vascular dementia [269], as adhered to by the Maastricht Memory Clinic, can be regarded as being accurate. 


\section{Psychiatric disorders in patients attending an outpatient memory clinic ${ }^{*}$}

\section{Introduction}

Deficits of memory and other cognitive functions in the elderly patient may indicate a variety of internal somatic, neurological or psychiatric disorders. Principally, the assessment of the memory disturbed patients involves two steps: first a syndromal diagnosis is made, e.g., "dementia", "delirium" or "amnestic disorder; second, it is investigated which factors produce that cognitive syndrome. Besides a comprehensive internal and neurological examination, a thorough psychiatric investigation is generally recommended $[54,167,207,281]$. Numerous organic disorders are known to cause cognitive deficits. In contrast, most studies mention major depression as the single functional cause $[38,82,108,179,241,290,328,329]$. Because it is not known whether, and to what extent, psychiatric disorders other than major depression are also accompanied by impaired cognition, the present study examined the psychiatric diagnoses of 430 consecutive outpatients attending a memory clinic.

\footnotetext{
*Adapted and translated from:Verhey FRJ, Jolles J, Ponds RWHM and Vreeling FW. Psychiatrische stoomissen bij patiënten van een geheugenpolikliniek. Nederlands Tijdschrift voor Geneeskunde 1993: 37:1054-1058
} 


\section{Patients}

Four hundred and thirty patients received a complete assessment in the memory clinic of the University Hospital of Maastricht between May 1986 and May 1991. Patients had been referred because of deficits or complaints about memory or other cognitive functions. These patients formed a heterogeneous group with respect to the severity of the cognitive impairment and the underlying aetiology. Table 1 shows the relevant demographic characteristics, the sources of referral and the cognitive syndrome (distinguished as dementia, no dementia, cognitive impairments objectified by neuropsychological tests, or subjective complaints, not objectifiable by neuropsychological tests).

Table 1. Characteristics of 430 patients referred because of memory deficits, by aetiological category.

\begin{tabular}{|c|c|c|c|c|c|c|c|}
\hline \multirow[t]{2}{*}{ characteristic } & \multicolumn{7}{|c|}{ aetiological category } \\
\hline & $\begin{array}{l}\text { primary } \\
\text { degenerativ }\end{array}$ & $\begin{array}{l}\text { cerebro- } \\
\text { evascular }\end{array}$ & $\begin{array}{l}\text { other } \\
\text { neurological }\end{array}$ & $\begin{array}{l}\text { internal- } \\
\text { somatic }\end{array}$ & $\begin{array}{l}\text { no } \\
\text { diagnosis* }\end{array}$ & unknown & total \\
\hline number of patients & 91 & 60 & 96 & 24 & 132 & 27 & 4,30 \\
\hline male/female & $36 / 55$ & $40 / 20$ & $60 / 36$ & $18 / 6$ & $71 / 61$ & $17 / 10$ & $242 / 188$ \\
\hline $\begin{array}{l}\text { age, mean (range) } \\
\text { referrer: } N,(\%)\end{array}$ & $72(50-88)$ & $72(35-88)$ & $56.6(16-81)$ & ) $57(27.87)$ & $56(21-85)$ & $61.7(23-85$ & 5) $61.7(16 \cdot 88)$ \\
\hline general practitioner & $39(43)$ & $15(25)$ & $20(21)$ & $10(41)$ & $50(38)$ & $18(67)$ & $152(35)$ \\
\hline neurologist & $28(31)$ & $31(51)$ & $59(61)$ & $8(33)$ & $44(33)$ & $5(19)$ & $175(41)$ \\
\hline psychiatrist & $18(20)$ & $7(12)$ & $12(13)$ & $3(13)$ & $34(26)$ & $3(11)$ & $77(18)$ \\
\hline $\begin{array}{l}\text { other } \\
\text { cognitive syndrome }\end{array}$ & $6(7)$ & $7(12)$ & $5(5)$ & $3(13)$ & $4(3)$ & $1(4)$ & $26(6)$ \\
\hline$N,(\%)$ & & & & & & & \\
\hline $\begin{array}{l}\text { dementia } \\
\text { nodementia }\end{array}$ & $88(97)$ & $41(69)$ & $17(18)$ & $4(17)$ & $2(2)$ & $0(0)$ & $152(35)$ \\
\hline objectifiedt & $3(3)$ & $19(31)$ & $73(76)$ & $17(71)$ & $77(58)$ & $20(74)$ & $209(49)$ \\
\hline not objectified $+\frac{t}{\psi}$ & $0(0)$ & $0(0)$ & $6(6)$ & $3(13)$ & $53(40)$ & $7(26)$ & $69(16)$ \\
\hline
\end{tabular}

* No diagnosis on axis III of DSM-III-R [7]

† Cognitive impairments objectified by neuropsychological tests

¥ Subjective cognitive complaints, without objectifable impaiments

With regard to the physical disorders or conditions described on axis III of the DSM-III-R (Diagnostic and Statistical Manual of mental disorders, 3rd edition [6] and, after 1987, 3rd revised edition (7]), the following alternatives were possible: 1) primary degenerative cause, most often probable Alzheimer's disease, 2) cerebrovascular cause, 3) neurological cause other than cerebrovascular, 4) somatic/ internal cause, also including intoxication and drug side-effects, 5) no diagnosis on axis III and 6) cause unknown. When there was more than one possible cause for the cognitive deterioration, the patient was classified according to the most 
probable cause. The most frequent diagnoses in category 3 ('neurological cause other than cerebrovascular') were epilepsia $(n=23)$, head trauma or whiplash trauma $(n=20)$, Parkinson's disease $(n=15)$, Huntington's disease $(n=7)$, and cerebral tumour $(n=6)$. Frequent diagnoses in category 4 ('somatic-internal') were drug side-effects $(n=6)$ and alcohol abuse $(n=4)$.

\section{Methods}

Every patient underwent an extensive neuropsychiatric and neuropsychological examination according to the standardized diagnostic procedure described in chapter 4. The major elements of this procedure are shown in table 2 .

Table 2. Standard diagnostic procedure in Maastricht Memory Clinic

with particular reference to...

history of the patient

history of the caregiver

neuropsychiatric history

somatic history

somatic investigation

neurological investigation

psychiatric investigation

neuropsychological investigation

ancillary investigations

rating scales complaints, first symptoms, course, insight into ones' deficits, subjective meaning, metamemory.

complaints, first symptoms, course, 'objective' data about social functioning, personality changes.

family history, early development, education, professional career, psychiatric and neurological history, head trauma, epilepsia, cerebrovascular accidents, neurotoxic agents.

standard, alcohol and drugs, nutrition

standard, signs of ageing

standard, release phenomena, behavioural neurology

consciousness, depression, anxiety, obsession, psychosis, personality, behavioural disorders.

psychometric tests, behavioural neurology [42] and tests derived from the information-processing paradigm [297]: memory recall, recognition, span, speed, susceptibility to interfering stimuli, complex information processing, apraxia, aphasia, agnosia, planning, evaluation, orientation, intelligence, reading, witing, calculating, personality questionnaires

blood chemistry, X-any, CT, MRI, EEG

Mini Mental State Examination [97] (for degree of cognitive dysfunctions); Hachinski's Ischemic Score [119] (for documentation of cerebrovascular factors); Hamilton Depression Rating Scale [120] (for degree of depressive symptoms); Blessed Dementia Scale [24] (for degree of behavioural dysfunctions); Global Deterioration Scale [255] (for global assessment of cognitive dedine) 
The neuropsychiatric assessment was carried out by an experienced neuropsychiatrist. The mean duration of the diagnostic assessment, including neuropsychological testing, was about 4 hours.

Psychiatric conditions, including dementia, were diagnosed according to the criteria of the DSM-III [6] and, after 1987, DSM-III-R [7]. For this study, only the axes I, II and III were taken into account (clinical syndromes, personality disorders and physical disorders, respectively). The diagnosis of 'probable Alzheimer's disease' or 'possible Alzheimer's disease' was made according to the criteria of the NINCDS-ADRDA work group [207]. Vascular dementia was diagnosed by using the DSM-III-R criteria and the ischaemic score of Hachinski et al. [119] (score $\geq 7$ ).

\section{Results}

Table 3 shows a matrix of the psychiatric diagnoses on axes I and II (rows) and the different axis III categories (columns). An organic mental syndrome was diagnosed in 189 patients and a functional mental syndrome was diagnosed in 152 patients. The remaining 89 patients did not have a psychiatric diagnosis according to the criteria of the DSM-III(-R),

One hundred and fifty-two of the 189 patients with organic mental syndromes had dementia, and in 53 of them (36\%) the dementia was complicated by additional psychopathology. Thirty-four of these patients had a depressive symptomatology. In two of these patients with dementia combined with depression, the dementia was presumed to be induced by the depression, which was apparent by a significant improvement of cognitive functioning after the start of antidepressive therapy. In the remaining 51 patients with complicated dementia, the psychopathology was not regarded as being the cause of the syndrome of dementia.

In 37 patients, an organic mental syndrome other than dementia was diagnosed, notably organic personality syndrome $(n=19)$ and organic mood syndrome $(n=16)$. Organic personality syndrome occurred with various disorders, e.g., Pick's disease, head trauma and alcohol abuse, whereas organic mood syndrome frequently occurred with Parkinson's disease $(n=6)$. One hundred fifty-two patients received together 159 diagnoses of a functional mental disorder on axis I or axis II. Most often, this involved a mood disorder, notably depression or dysthymia (38\% and $25 \%$, respectively); dysthymia, previously called neurotic depression or personal depression, is a condition which tends to be chronic, in which the mood is less severely disturbed and in which there are fewer somatic symptoms than in major depression [7]. A functional mental disorder other than a mood disorder was diagnosed in 59 patients (38\%). This especially involved adjustment disorders, personality disorders and anxiety disorders. In 101 patients of the 152 with a functional mental disorder.(66\%), the cognitive complaints could be objectified by neuropsychological testing; in the remaining patients, performance in the neuropsychological tests was normal. 
Table 3. Relationship between psychiatric diagnoses (on axes I and II of the DSM-III(-R) [7] and relevant physical disorders (axis III) in 430 patients attending a ourpatient memory clinic .

aetiological category of axis III

\begin{tabular}{|c|c|c|c|c|c|c|c|}
\hline $\begin{array}{l}\text { diagnosis } \\
\text { on axes I and Il }\end{array}$ & $\begin{array}{c}\text { primary } \\
\text { degenerative } \\
(\mathrm{n}=91)\end{array}$ & $\begin{array}{l}\text { cerebro- } \\
\text { vascular } \\
(n=60)\end{array}$ & $\begin{array}{c}\text { other } \\
\text { neurological } \\
(n=93)\end{array}$ & $\begin{array}{l}\text { internal- } \\
\text { somatic } \\
(\mathrm{n}=24)\end{array}$ & $\begin{array}{c}\text { no } \\
\text { diagnosis* } \\
(n=132)\end{array}$ & $\begin{array}{l}\text { unknown } \\
(n=27)\end{array}$ & $\begin{array}{c}\text { total } \\
(n=430)\end{array}$ \\
\hline axis Ior II & 1 & 12 & 34 & 7 & 15 & 20 & 89 \\
\hline
\end{tabular}

organic mental disorder

dementia,

$\begin{array}{lccccccc}\text { uncomplicated } & 55 & 27 & 13 & 4 & - & . & 99 \\ \text { with depression } & 20 & 9 & 3 & - & 2 & - & 34 \\ \text { with delusions } & 2 & 1 & - & - & - & - & 3 \\ \text { with delirium } & - & 1 & - & - & - & - & 1 \\ \text { with anxiety } & 6 & 2 & - & - & - & - & 8 \\ \text { with behavioural disorder } & 3 & 1 & 1 & - & - & - & 5 \\ \text { with obsessions } & 2 & - & - & - & - & - & 2 \\ \text { organic personality syndrome } & 3 & 2 & 10 & 3 & - & 1 & 19 \\ \text { organic mood syndrome } & 2 & 3 & 10 & 1 & - & - & 16 \\ \text { organic delusional syndrome } & - & - & - & 1 & - & - & 1 \\ \text { delinum } & - & - & - & 1 & - & - & 1\end{array}$

functional mental disorders

depression

15

$5 \quad 50 \quad 61$

dysthynia

- 35

39

adjustment disorder

9

23

personality disorder

11

anxiety disorder

phobia/ obsessive

compulsive disorder

dyslexia

mental retardation

somatoform disorder

16

18

1

3

6

atypical psychosis

$\begin{array}{lll}4 & \cdot & 4 \\ 2 & - & 2 \\ 1 & \cdot & 2 \\ 1 & - & 2 \\ 2 & - & 2\end{array}$


Relationship between psycbiatric symptoms, cognitive impairments and underlying disorder

The cognitive symptoms, the psychiatric condition and the underlying disorder may be interrelated in several ways. The following five case histories illustrate this.

Patient A, a 72-year old man, was referred for differentiation between dementia and depression. Investigation yielded apparent signs of Parkinson's disease. Moreover, the patient had progressive dementia with deficits of memory retrieval, abstract thinking, excessive cognitive slowing and depression, for he could give no reason. Low doses of antidepressant medication caused confusion in the patient. In contrast, levo-dopa therapy improved the symptoms of Parkinson's disease and depression, although the cognitive symptoms remained unchanged.

Comment. The psychiatric symptoms as well as the cognitive symptoms in this patient were probably both caused by an underlying brain disease [62].

Patient $B, a 63$ year old man, was also referred to examine whether he suffered from dementia or depression. After his daughter's death 2 years ago, he demonstrated memory deficits, apathy, disorientation and he neglected his personal hygiene. He was found to fulfil the criteria of dementia, and more specifically, with characteristics of the subcortical type. Formal neuropsychological testing appeared hardly possible due to a lack of motivation. The patient responded with numerous "don't know" answers on questioning. Antidepressant therapy and supportive psychotherapy resulted in a significant improvement of both the affective and the cognitive symptoms.

Comment. In this patient, a primary psychiatric disorder (i.e., depression) resulted in cognitive impairments, severe to warrant the diagnosis dementia. This condition, previously termed "pseudodepression, can be explained in psychological terms, e.g., lack of motivation $[48,78]$, as well as by biochemical mechanisms $[78,343]$. However, this condition of so called depression-induced dementia eventually turns out to be the very first manifestations of a primary brain disease, notably Alzheimer's disease, in more cases than would be expected on the basis of chance.

Patient $\mathrm{C}$ was a 70 year old woman whose brother had been diagnosed as suffering from Alzheimer's disease 2 years before. From the start she was terrified by that diagnosis: "I have the same disease in my head as my brother, and that's very bad!". She reported panic attacks when confronted with her cognitive impairments, and she showed signs of panic on neuropsychological testing. The diagnostic assessment revealed mild dementia caused by Alzheimer's disease, with memory deficits and slight aphasia dominating the clinical picture.

Comment. Besides the primary organic mental syndrome, there were also signs of a psychological reaction: cognitive symptoms resulted from a brain disease, but her intact insight into her deficits led to the feeling of fear. Such situations can exist especially in the early stages of Alzheimer's disease (see also chapter 11, [321]). 
Patient $D, a 56$ year old book-keeper, has been fired because of signs of 'surménage'. He had complaints of depression, poor concentration, irritability and abnormal fatiguebility. The classification according to the DSM-II-R criteria was dysthymic disorder. He had a history of two mild head injuries and two operations with general anaesthesia many years ago. On neuropsychological testing, no memory impairments could be objectified, but he performed poorly on tests measuring susceptibility for interfering stimuli and cognitive speed. After 2 years, he reported no signs of depression, but his neuropsychological performance remained unchanged.

Comment. Patients with this type of profile of complaints and neuropsychological performance are encountered quite frequently in our memory clinic. Although these complaints may often be interpreted in psychological terms [2], biological factors may also be relevant with regard to their pathogenesis. Earlier studies showed that a group of patients with dysthymia had a higher prevalence of previous exposure to potentially brain damaging influences, such as use of alcohol, brain trauma and anaesthesia, than normal age-matched and education matched controls $[149,320]$. These so called "biological life events" are correlated with decreased cognitive functioning with increasing age. It is often unclear whether the cognitive symptoms result from the psychiatric condition, or vice versa, i.e., whether the cognitive impairments lead to symptoms of depression. The fact that the signs of depression in this patient had improved after 2 years, in contrast to the cognitive aspects, suggests a primary role of the cognitive impairments. This will be discussed in more detail in chapter 8 of this thesis.

Patient E, a 62 year old woman, worried about her memory, although neuropsychological testing revealed no abnormal performances. Her personality could be described as obsessive. Moreover, she was very afraid of becoming demented.

Comment. There were no signs of an organic disorder or of any cognitive deficit in this patients. Her complaints were probably related to worries about Alzheimer's disease and the. extreme demands that she made on her own memory.

\section{Discussion}

Although affective disorders (major depression and dysthymia) formed the greater part of the functional psychiatric diagnoses of the group that was studied, memory complaints also occurred together with many other psychiatric conditions. In most other studies, however, depression is seen as the single functional cause of cognitive impairments. In eight publications on the causes of memory disorders in patients from comparable memory clinics, involving 998 patients, psychiatric disorders other than dementia were diagnosed in 133 patients. Major depression was the main disorder, accounting for 112 patients $(84 \%)[38,82$, $108,179,241,290,328,329]$. In our sample, however, primary and secondary affective disorders together formed not more than $60 \%$ of all psychiatric conditions. Notably, adjustment disorders, anxiety disorders and personality disorders also occurred with the 
cognitive symptoms. As illustrated by the five case histories described above, the relationship between the psychiatric condition and the cognitive problems can be more complex than just a linear causal one and has to be elucidated by individual psychiatric assessment.

It could be argued that the MMC is a specialized neuropsychiatric department, and therefore some selection bias may have influenced the outcome of our study. As was shown in chapter 4 , however, in almost half of the patients (44\%) the psychiatric condition was not recognized until assessment took place in our memory clinic [316]. As a consequence, the existence of a psychiatric disorder in these patients could have led to selection bias. Thus, the results of the present study are probably relevant to the more general neurological, psychiatric or geriatric practice.

There are several possible explanations for the discrepancies between the results of our study and those of most other studies. The MMC partly functions as a tertiary reference centre; about half of the patients had been referred by their general practitioner. Sources of referral of other studies show considerable variation: some show quite a few self-referrals [241], others referrals by general practitioners $[38,82,108]$, whereas the majority of patients in other studies were referred by medical specialists [179]. Moreover, studies differ substantially with regard to the mean age of the patients (varying from 57.7 years [179] to 75.8 years [290]), as well as to the proportion of patients with dementia (varying from 51\% [241] to $94 \%$ [179]). In study, the mean age was 61.7 years, and $35 \%$ of the patients fulfilled the criteria of dementia. Each of these aspects influences the prevalence of psychiatric disorders, although it is difficult to account for the exact extent and the direction of the selection bias in terms of these factors.

Additionally, the differences between the various studies could be explained by differences in the way the assessment was carried out. Thus, the psychiatric evaluation in some studies is explicitly restricted to checking whether symptoms of depression are present or not $[38,108]$. In other studies, psychiatric evaluation only takes place when other specialists suspect depression to be present [82], or the diagnostic criteria for psychiatric disorders are not stated clearly $[241,290]$. Therefore, it is plausible that the relatively high number of patients with depression and the low number of patients with non-affective psychiatric disorders could result from an incomplete psychiatric examination. This would probably lead to an underestimation of the prevalence of non-affective psychiatric disorders and, as a consequence, potential possibilities for treatment will not be used or will be used inappropriately.

\section{Conclusion}

Athough affective disorders are the most frequent psychiatric disorders that accompany memory complaints, several other psychiatric conditions are also related to these complaints. Therefore, psychiatric assessment of patients with memory disorders should not be restricted merely to looking for depressive symptoms. 


\section{Cognitive dysfunctions in middle-aged subjects with late-onset dysthymia attending a memory clinic*}

\section{Introduction}

It is well established that many cognitive abilities deteriorate with age $[57,145,247]$. Elderly people aged 65 and above perform tasks involving new information, planning of new activities and effortful, controlled processing relatively $[28,57]$. Above all, there is a general slowing of behaviour with increasing age [277]. There is relatively less information about the effect of age on cognitive functioning in middle-aged subjects although complaints about memory, attention and concentration among middle-aged persons are very common $[59,176,247]$. It is relevant in this respect that a significant part of the patients in specialized memory clinics [38, $39,241,316]$ is formed by middle-aged subjects with complaints about memory, attention and concentration. These patients often do not fit well into the available classifications, e.g. DSM III-R. Recent experimental evidence suggests that the cognitive performance of middle-aged subjects might already be inferior to that of young adults [132-134]. However, information on the relation between cognitive complaints and objective deficits in middle-aged subjects is sparse.

There are only few diagnoses that cover middle-aged persons with complaints about memory related functions, loss of energy and mood disturbances. When accompanied by mild but persistent affective complaints, a diagnostic classification of late-onset dysthymia is the most relevant. According to the Diagnostic and Statistical Manual for Mental disorders, revised 3rd edition, (DSM-III-R) [7], a diagnosis of dysthymia is made when there is no major depression, when two or more of a variety of nonspecific complaints are present and when the symptoms are chronic, i.e., lasting two years or more. The complaints mentioned in DSM III-R

*JJolles, FRJ Verhey, PJ Houx and EJ Reyersen wan Buuren, submitted for publication. 
are: chronic disturbance of mood (depressed and/or irritable), poor appetite, insomnia, low energy and fatigue, feelings of hopelessness, as well as cognitive complaints, such as poor concentration and difficulty making decisions. Subtyping of DSM-II's dysthymia is allowed for age of onset (before and after the age of 21) and the relationship to preexisting chronic axis I or III disorders (primary or secondary type). Other relevant diagnostic categories for memory complaints in elderly subjects concern Benign Senescent Forgetfulness (BSF) [171] and Age-Associated Memory Impairment (AAMI) [59]. BSF originally describes memory complaints in the senium. The concept is ill defined and its nosological status has not been established yet. A diagnosis of AAMI essentially pertains to normal aging and is only possible when problems in the cognitive domain are prominent and when no major neurological or psychiatric diagnosis can be made, documented for example by a score on the Hamilton Depression Rating Scale [120] of less than 13.

There is little knowledge about the nature of the cognitive dysfunctions that might be present in middle-aged subjects who complain about memory and who have mild but persistent mood changes. In the last decade, information processing deficiencies have been proposed in relation to affective disorders. In this view, every stage and type of information processing has implications in determining aspects of cognitive dysfunctioning in depressive people [211]. Attentional and motivational dysfunctions, encoding and rehearsal problems, and alterations in decision making and response patterns can thus be present. Weingartner [330] linked the cognitive dysfunctions in depression to alterations in motivation, appreciation and drive, resulting in disturbances of all kinds of effort-demanding cognitive processes as formulated in terms of the influential theory of Shiffrin and Schneider [283]. The problems that these depressive patients experience are especially evident in tasks involving sustained attention, effort, time constraints and increased memory load. Similar deficits are seen in the various subtypes of major depression [201]. However, it is not known whether milder forms of depression - including dysthymia - are also characterised by these cognitive deficits. In addition, little is known about the relative contribution of the factor age. The literature contains conflicting reports regarding the negative effects of depression and age on cognitive functioning. For instance, some authors argue that depression and age have no effect [223] whereas others state that they have an adverse effect [251].

The present study investigates middle-aged dysthymic subjects (aged 40-65) who seek help for their cognitive complaints in a specialized memory clinic. Such health care facilities have been set up in recent years primarily for the early diagnosis and treatment of elderly subjects with dementia, but few facilities exist for the diagnosis and treatment of memory complaints in non-demented presenile and senile persons $[59,316]$. The major question was whether these patients are characterised by objectively assessed decreased performance in neuropsychological tests as compared to age-matched controls. The relevance of finding cognitive dysfunctions in this particular group is that dysthymia is usually seen from a psychopathological or psychosocial perspective, e.g, as a residual state of major depression, as a personality disorder or neurotic disorder $[2,164,166,332]$ and not from the perspective of physiological aging. A clinical experiment was performed in which the performance of 25 dysthymic patients was compared to that of individually age-matched control subjects in a number of neuropsy- 
chological tests. The selection of the cognitive neuropsychological tests was based upon earlier work in which impairments were found in particular stages of information processing in AAMI patients $[31,330]$. Our main hypothesis was that the patients would show a deficit in tasks involving effortful, time consuming processing. Tasks requiring mainly automatic processing would not distinguish the two groups from each other.

\section{Materials and methods}

Patients

From 430 outpatients of the Maastricht Memory Clinic of the University Hospital of Maastricht, the Netherlands, 100 met the DSM III-R criteria for affective disorder, i.e. 61 had major depression and 39 dysthymia. Only the 25 patients with dysthymia and aged between 40 and 61 years were included in this study. The mean age was 49.1 year (sd 5.2, range 41-61), there were 19 male and 6 female. The patients had been referred to this department by general practitioners, psychiatrists and neurologists because of problems concerning cognitive functioning -most often problems in memory and concentration. The diagnostic procedure has been described elsewhere in detail [316]. In brief, this involved a standardised psychiatric interview, a physical and neurological examination, an extensive neuropsychological investigation and laboratory investigations. Diagnostic classification was made by a neuropsychiatrist according to the DSM-III-R [7]. Patients were excluded when a physical, neurological or major psychiatric disorder - including dementia, major depression, double depression or adjustment disorder- was diagnosed, or when they used medications with known psychoactive effects. A Hamilton Depression Rating Scale (HDRS) [120] was administered to all patients to obtain an indication for the degree of the depressive symptoms, but no cut-off was used as a diagnostic criterion. The mean HDRS-score was 14.1 (sd 4.9, range 5-26) The mean score on the Mini-Mental State Examination was 28.8 (sd 1.4, 26-30). The cognitive and mood complaints existed for a mean of 4.6 years (sd 3.6, range 16.4-1.5 years) and the start of the complaints was gradual. Although the DSM-III-R requires officially a duration of complaints of at least 24 months, in this sample five subjects were included who had complaints between 18 and 24 months.

\section{Control subjects}

The control group of 25 volunteers was drawn from a larger population of subjects who were normal and healthy according to regular criteria used in gerontological research $(n=247)$. Control subjects were selected by means of matching to the individual patients with respect to age ( \pm 3 years), gender and level of education ( \pm 1 point). The latter was assessed by a Dutch scoring system [314], which comprises a scale ranging from 1 (primary education not finished) to 7 (university degree). The two groups thus consisted of twenty-five matched pairs. The procedure for recruitment of the controls has been described elsewhere [134]. They underwent the same procedure of routine physical and neurological examination and neuro- 
psychological investigation as the index group. All subjects were free of significant somatic, neurological and/or psychiatric disorders. HDRS scores from the controls were not available, but all denied complaints of lowered mood, nor did they report significant problems of memory or concentration. The control subjects can be regarded as a representative selection of the middle aged population [132].

\section{Neuropsycbological Tests}

The Digit Span (forward) is a subtask in the Wechsler Adult Intelligence Scale [183] and is taken as a measure of primary memory for auditory-verbal material.

The Auditory Verbal Learning Test is a Dutch version of the Rey Auditory Verbal Learning Test $[31,183]$. The test consists of one list of 15 monosyllabic and concrete nouns in Dutch, which are presented in 5 trials by means of a tape recorder. The nouns ("tree, bread, dog, knee,...") occur very frequently and are acquired earlier in life. Items were presented in the same sequence at a rate of one per two seconds. Each trial ends with a free recall of the words (free immediate recall). After a period of 20 minutes following the fifth trial, the subject is requested to recall as many words as possible (free delayed recall). A yes/no recognition test, consisting of the 15 former words and 15 new but similar words is given after the delayed recall test. The variables used are: the total number of correct words over the 5 trials as a measure of learning capability; the number of correct words on delayed free recall as a measure of retrieval from long-term memory; the number of correct responses on delayed recognition as a measure of memory consolidation; the total number of errors and double responses as a measure of the efficiency of the different memory processes and of self monitoring and evaluation.

The Revised Trail Making Test (R-TMT; [324]) is an adapted version of the task described by Reitan [183], which is a test of conceptual and visuomotor tracking. The R-TMT consists of three subtasks. In each subtask, the subject has to connect small circles with digits or letters in the right order on an A4 sheet of paper. The subject is requested to do this as quickly as possible. In the first subtask (A), the subject must draw lines to connect consecutively numbered circles (1-26). In subtask B, the lettered circles A-Z have to be connected, and subtask C consists of 26 circles containing 13 digits (1-13) and 13 letters (A-M). The subject is asked to alternate between the two sequences (1-A-2-B etc). The layout of the three subtasks is similar. The variables are: response time in version $A$ and $B$ as a measure of conceptual tracking. The times needed to complete each subtask are used in the formula: $t C-1 / 2(t A+t B)$ as a measure of concept shifting ability for task $\mathrm{C}(\mathrm{t}=$ time in seconds).

The Stroop Colour Word Interference Test [183] is a perceptual interference task and consists of three subtests. The test examines the speed at which colour names are read (subtask 1) and the speed to name colours (subtask 2). Subtask 3 involves colour names again, but the printing ink is different from the colour name. The speed at which the printing ink of the words is named is taken as the test variable. The interference score results from the subtraction of the time needed for subtask 2 from that of subtask 3 . 
The Memory Scanning Task (MST; paper and pencil version, see [31] for description) is based upon the Sternberg paradigm [297]. Briefly, a set of 1,2,3 and 4 letters has to be memorised. The subject is asked to search for these letters on a test form consisting of 144 capital letters typed in a $12 \times 12$ matrix, 4 spaces apart (A4 format). The subject has to mark the targets with a pencil. A sixth of these letters are items from the memory set, the rest are distracter letters. There is a practice run with the symbol '\%' as memory set before part one (set size 1). For each of the subtasks, both time and the number of errors of omission and false positives are noted. The intercept of the time/sersize function is taken as a measure of general speed of information processing and the slope is a measure of the efficiency of the memory-scanning stage.

\section{Results}

The test performance of both groups was analysed with a multivariate analysis of variance (MANOVA) with or without repeated measurement for consecutive subtasks. Variables that deviated markedly from a Gaussian distribution were transformed according to Stevens [298]. $P$-values lower than 0.05 were regarded significant.

Multivariate analysis yielded a significant group-effect (Wilks lambda: $0.39, F=3,75$, $p=0.01$ ). Therefore, separate univariate tests per cognitive parameter were carried out. Mean performances on the various tests are shown in figures $1 \mathrm{a}, \mathrm{b}, \mathrm{c}, \mathrm{d}$ and in table 1 . No group differences were observed in auditory immediate memory (Digit Span). With regard to the accuracy with which the various tasks were performed, reflected by the number of errors, corrections, omissions and double responses, significant differences were only found in the memory scanning test and Stroop-III.

A repeated measures analysis of variance revealed a significant group-effect for the AVLT : $F(1,48)=31.61, p<.001$. The mean maximum score (usually at the 5 th trial) was $22 \%$ lower in dysthymic subjects than in controls $(9,76$ vs. 12,6; MANOVA $F=28.9, p<0.001)$. Furthermore, delayed recall and recognition after 20 minutes were both poorer in dysthymic subjects (respectively, $F(1,48)=34.17, p<0.001$ and $F(1,48)=15.2, p<0.001$ ). Repeated measures analysis of variance of the Trail Making Test (R-TMT, fig.1b) revealed a significant group effects $(F(1,45)=5.68, p<0.05)$, which means that visuomotor tracking is inferior in dysthymic subjects. There was no significant group by time-interaction. This indicates that dysthymic subjects did not need more time to shift between the concepts of digit and letter sequences. Repeated measures analysis of variance of the Stroop test showed a significant group effect $(F(1,48)=13.7, p<.001)$ and a group by time-interaction $(F(2,47)=4.86, p<.01)$. It appeared (fig 1c) that dysthymic subjects were slower in reading, colour naming, and in the interference task. Further subgroup analysis showed that this was mainly caused by the fact that the dysthymic patients, compared to controls, needed much extra time for the interference task. The results of the paper-and-pencil memory scanning task (fig 1d) show that the dysthymic subjects were generally slower in processing visuomotor information as measured by the lettersubtask $(F(1,44)=11,72, p<0.01)$. Moreover, the slope of the total time needed per test sheet, plotted against the number of items that have to kept in short-term memory, was 
steeper in dysthymic subjects. This suggests that they have slower memory search processes, especially with increasing memory load.

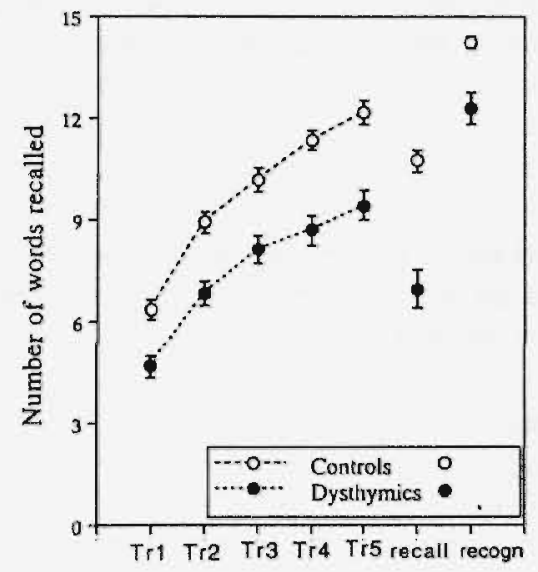

Figure 1a. Auditory Verbal Learning task

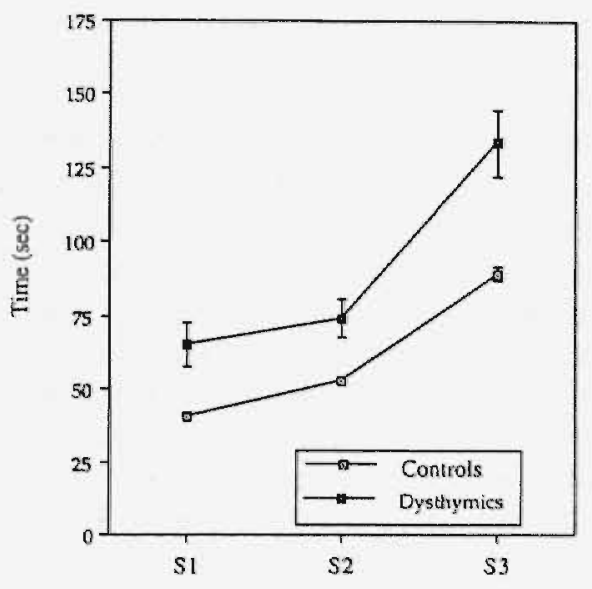

Figure 1c. Stroop Color-Word-Interference Test

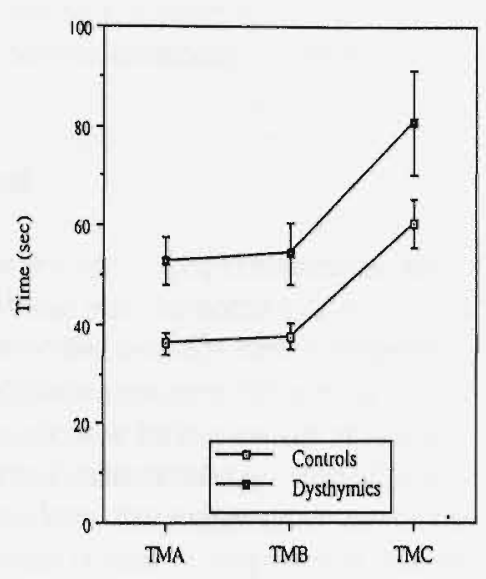

Figure $1 \mathrm{~b}$. Trail Making A, B and C.

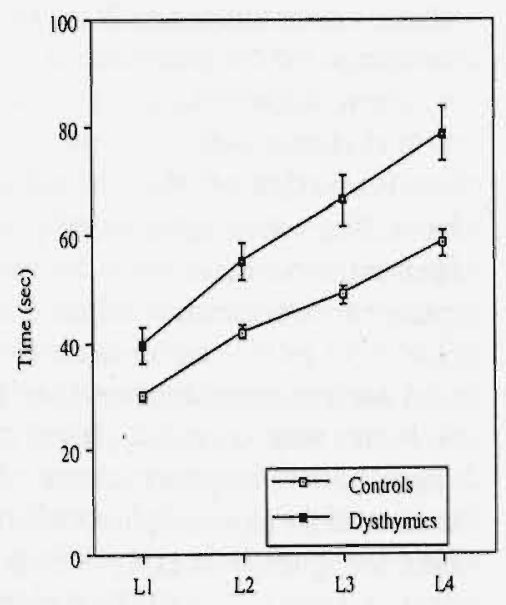

Figure 1d. Memory Scanning Test 
Table 1. Psychometric differences between dysthymic patients and controls $(\mathrm{N}=25)$ : Performance on main test variables

\begin{tabular}{|c|c|c|c|c|c|c|c|c|}
\hline & \multicolumn{3}{|c|}{ Dysthymics } & \multicolumn{3}{|c|}{ Controls } & \multirow[b]{2}{*}{ F } & \multirow[b]{2}{*}{$p$} \\
\hline & $\mathrm{N}$ & mean & sd & $\mathrm{N}$ & mean & sd & & \\
\hline Digitspan & 25 & 5.3 & 1.0 & 25 & 5.7 & 1.1 & 1.1 & n.s. \\
\hline \multicolumn{9}{|l|}{ Auditory Verbal Learning Test } \\
\hline Total in 5 trials & 25 & 37.7 & 8.3 & 25 & 49.0 & 5.7 & 27.7 & 0.001 \\
\hline Delayed recall & 25 & 7.0 & 2.8 & 25 & 10.8 & 1.6 & 27.9 & 0.001 \\
\hline Delayed recognition & 25 & 12.3 & 2.4 & 25 & 14.2 & 0.8 & 18.7 & 0.001 \\
\hline \multicolumn{9}{|l|}{ Stroop Color Word Test } \\
\hline Stroop-I (reading) & 25 & 65.1 & 38.0 & 25 & 40.2 & 4.7 & 10.6 & 0.01 \\
\hline Stroop-II (color naming) & 25 & 74.2 & 32.2 & 25 & 52.6 & 7.2 & 10.8 & 0.01 \\
\hline Stroop-III (interference) & 25 & 133.7 & 56.2 & 25 & 89.5 & 12.7 & 14.7 & 0.001 \\
\hline \multicolumn{9}{|l|}{ Trail Making Test } \\
\hline Trial A & 25 & 52.8 & 23.6 & 25 & 36.1 & 11.5 & 10.3 & 0.01 \\
\hline Trial B & 22 & 54.3 & 31.2 & 25 & 37.7 & 12.7 & 6.0 & 0.05 \\
\hline Trial C & 23 & 80.8 & 52.1 & 25 & 60.4 & 24.6 & 33 & n.s. \\
\hline \multicolumn{9}{|l|}{ Memory Scanning Test } \\
\hline "\%" & 24 & 33.8 & 17.1 & 25 & 24.7 & 4.0 & 9.9 & 0.01 \\
\hline 1 letter & 24 & 39.7 & 17.5 & 25 & 30.0 & 4.9 & 5.8 & 0.05 \\
\hline 2 letter & 24 & 55.2 & 17.2 & 25 & 42.0 & 6.8 & 10.3 & 0.01 \\
\hline 3 letter & 23 & 66.3 & 23.1 & 25 & 48.9 & 8.7 & 9.3 & 0.01 \\
\hline 4 letter & 21 & 78.6 & 25.4 & 25 & 58.4 & 11.9 & 12.6 & 0.001 \\
\hline intercept & 21 & 39.9 & 18.7 & 25 & 31.0 & 5.0 & $Z=1.65$ & $\mathrm{n} . \mathrm{s}$ \\
\hline slope & 21 & 12.7 & 6.2 & 25 & 9.2 & 3.3 & $Z=2.26$ & 0.02 \\
\hline
\end{tabular}

Pigures represent means and standard deviation in time (seconds), except for Verbal Learning Test, in which the number of words recalled are presented. Wilcoxon test for results on intercept and slope of memory scanning test; repeated measures analysis of variance is applied for all other variables.

\section{Discussion}

The major question of the present study was whether middle-aged dysthymic subjects who seek help from a memory clinic with complaints about memory are characterised by cognitive deficits, as assessed by their performance in neuropsychological tests. It appeared that the performance of the dysthymic subjects was significantly inferior to that of age-matched controls in tests of secondary memory, such as the immediate and delayed recall as well as the delayed recognition on a verbal learning task. In contrast, primary memory, reflected by the 
score on the Digit Span, did not differentiate between the two groups. Differences were found in the performance of information processing tasks in which task variables were manipulated in order to change the cognitive complexity of the task: significant differences were found for those subtasks that had comparatively high cognitive complexity. For instance, the dysthymic subjects needed significantly more time with increasing memory load to perform the Memory Scanning Task, as reflected by a significantly increased slope for this task (Fig.1d, Table 1) than the controls did. In addition, the patients needed more time for the Colour Word Interference Subtask (Stroop III). In addition to the findings on the complex subtasks, significant differences were found with those subtasks of Memory Scanning Task, Stroop and R-TMT that measure the general speed of information processing. Thus, dysthymic subjects were somewhat slower than the controls in the Memory Scanning Task, in colour naming and word reading in the Stroop and in visuomotor tracking in the Revised Trail Making Test.

The results can be taken to indicate that the dysthymic subjects, apart from being slower, had a particular problem with effort-demanding processing. This type of task requires 'controlled processing' as opposed to 'automatic processing', to use the terms proposed by Shiffrin \& Schneider[283]. The results suggest that dysthymic subjects make more use of a controlled processing strategy, which costs them more time and energy. This is exactly the complaint that many of these patients have, namely that they are not able to perform simple tasks as automatically as they used to; that everything costs energy and effort; that they feel unable to perform complex tasks involving several actions parallel to each other and/or in a short time period. Similar conclusions were reached in studies with depressive subjects (e.g., [330]). The present findings appear to provide an objective counterpart of the subjective complaints of the dysthymic subjects investigated. More rearch is needed to investigate in more detail the performance on tasks which are effort demanding in relation to the subjective complaints, since cognitive effort may be confounded with limited time for performance in speeded tasks.

Alternative explanations for the differences in test performance between the dysthymic subjects and controls concern differences in motivation, age and intelligence. However, the dysthymic subjects were in general highly motivated to perform well because of a subjective fear of dementia for which they sought help in the memory clinic. Thus, it is not likely that differences in motivation account for the cognitive group effects. Intelligence Quotient had not been assessed from the controls and thus, possible differences in intelligence to explain for the findings of this study can not be completely ruled out. However, this explanation seems highly unlikely since the groups were matched for level of education. By matching the two groups for age, we controlled at least for chronological age.

With respect to the relationship between the cognitive impairments and dysthymia, there are several possibilities. In the first place, it may be that the cognitive dysfunctions are primary and result into disturbed adaptation in social and professional life, which in tum leads to a chronically depressed state and other symptoms of dysthymia. If cognitive impairment is primary, the question is by what it is caused. One possibility as to aetiology is that an abnormal aging process is involved. Some of the middle-aged dysthymic subjects could be in a very early phase of a neurodegenerative disease such as Alzheimer's Disease. Patients with major depression and cognitive deficits have been shown to have an increased risk to develop 
dementia $[78,172,252]$. Longitudinal research will be carried out with the subjects of this study to check this notion. It is of interest in this respect that the cognitive deficit profile of the dysthymics in this study is similar with the profile of cognitive decline commonly reported for normal aging in persons over 65 years [134]. Another possibility is that other, as yet not identified, biological factors are responsible for the decreased performance of the middle-aged subjects. There is some evidence that dysthymics differ from normal subjects and from patients with major depression with respect to the dexamethasone suppression test, response on stimulation with 'Thyrotropin-Releasing Hormone (TRH), respons to antidepressive drugs and several other biological aspects. $[135,136]$ These differences may reflect an altered brain functioning, which leads to poor cognitive performance. Interestingly, several conditions have been identified in which a minor event to the brain (e.g., very mild brain trauma, longstanding exposure to industrial agents or recovery from infectious diseases) yields a neurasthenic syndrome -a concept that is covered by the DSM criteria for dysthymia- in which no major physical or neurological abnormalities can be identified [187]. The findings of the present study support this possibility, which has also been under consideration in other studies [132, 133]. These indicate that such biological life events could accentuate the effect of normal biological aging and become manifest in middle-age when the normal biological aging process becomes evident. The second possibility with regard to the aetiology is that the cognitive dysfunctions might be an inherent part of the dysthymic disorder, parallel to or even secondary to mood changes, insomnia, low energy, fatigue etc. that are caused by particular psychosocial events. A life event as a psychological cause for dysthymia was ruled out by the exclusion of adjustment disorders, but it is theoretically possible that the patients responded in a neurotic way to the physiological changes associated with normal biological aging. However, the results show that the cognitive complaints could be objectified, which precludes a mere neurotic perception of normal aging as a plausible explanation. Interestingly, the patients in the present study had functioned well and esteemed life in a positive way both from a social and professional point of view in the period before the dysthymia had started $(1.5$ to 6 years before the start of the present study), which makes an underlying characterological disorder unlikely.

A third possible way to conceptualize the relationship between cognitive symptoms and dysthymia could be that the cognitive complaints as well as some of the dysthymic symptoms, such as poor appetite and insomnia, are both related to aging itself. This possibility seems improbable, since the results showed significant differences between the age-matched groups, all subjects were free from significant somatic problems and since the symptoms above occur more frequently in the senium, but probably not in subjects aged between 40 and 65 . However, in older patients dysthymia and the process of aging may share some common characteristics. More longitudinal studies into the course of the different symptoms can elucidate the relationship between affective and information processing features and will be carried out in our department. 


\section{Conclusion}

The present study shows that middle-aged dysthymic subjects who seek help from a memory clinic for complaints about memory-related functions are characterized by cognitive deficits when compared to age-matched controls. The results resemble those found recently for information processing deficits in depression. The question whether the cognitive dysfunctions found in the memory clinic population can be generalised to all dysthymic subjects can not be answered in the present study. We have found that middle-aged patients suffering from dysthymia -or at least a subgroup of this condition- are characterised by information processing deficits in addition to the known psychiatric symptoms. The data may provide new insights into the nature of a disease which, although generally considered as mild, may lead to severe handicaps in normal daily life. In addition, the data draw attention to the importance of the factor age in the evaluation of mild cognitive complaints. New models and techniques aimed at coping styles and cognitive rehabilitation $[50,236,260,337]$ can be based upon a thorough knowledge of the cognitive deficits described in the present study. 


\section{A two-year follow-up of non-demented patients attending a memory clinic*}

\section{Introduction}

The differentiation of early Azheimer's disease from normal age-associated memory impairment is problematic when the clinical symptomatology is below the threshold for dementia. There is no consensus on the diagnostic criteria of the borderline states between normal ageing and frank dementia, because there is limited knowledge about which are the crucial symptoms that accurately predict the development of dementia. A strategy has been recommended by which a range of variables are followed longitudinally, in the hope that time will show which symptoms are characteristic for the prodromes of dementia [124].

In this chapter we describe an ongoing longitudinal project at the Memory Clinic of the University Hospital of Maastricht. The aim of this project is to collect data on a cohort of middle-aged and older patients who seek help for cognitive complaints in a specialized memory clinic, in order to establish the clinical characteristics of the prodromes of dementia. A related goal of this project was to investigate the relationship between cognitive and affective symptoms by following their course over time. In this chapter, the experiences and difficulties related to this approach are discussed. The first results of this longitudinal study will be reported.

\footnotetext{
"Verhey FRJ Rozendaal N, Houx P, Ponds RWHM, Vreeling FW and Jolles J, submitted
} 


\section{Patients and methods}

\section{Patients}

The cohort of this study consisted of 65 patients who had been examined in the Maastricht Memory Clinic of the University Hospital of Maastricht (MMC), The Netherlands before May 1990. Patients were included in this study 1) when they had completed the diagnostic procedure; 2) when they were 40 years or older; 3) when they were not demented, according to the criteria of the DSM-III-R [7] or a score on the Mini-Mental State Examination (MMSE) [97] of 24 or less; and 4) when no organic or somatic causes for the cognitive complaints could be established after careful assessment as described elsewhere [316]. Because depression or anxiety may be early signs of Alzheimer's disease [172, 252], patients with these disorders were not excluded in this study. If treatment for a psychiatric disorder was considered necessary, this was performed after the initial assessment according to regular practice. The patients were asked to discontinue medications with known psychoactive effects a few days before the follow-up assessment. The patients had been referred by a general practitioner or a medical specialist (neurologist or psychiatrist) primarily because of their cognitive complaints, and not primarily because of a psychiatric condition. There were no self-referrals.

Patients fulfilling the inclusion criteria were contacted 2 years \pm 3 months after the initial investigation in the MMC and were invited to attend follow-up appointments. When patients declined to participate, they were interviewed by telephone about the reason for their refusal and about any changes since their visit to the MMC two years ago.

\section{Initial assessment}

The initial assessment of each patient was carried out by a neuropsychiatrist and a neuropsychologist and included a detailed history provided the patient and a significant other, a mental status (including psychiatric) examination, a physical (including neurological) examination and ancillary examinations such as laboratory tests and a CT scan of the brain. Psychiatric disorders were diagnosed with DSM-III-R criteria [7]. The Mini-Mental State Examination (MMSE [97]) was administered to 55 patients (this test was not part of the routine MMC procedure in the first months of the clinic, resulting in missing values for the first 10 patients). Hamilton's Rating Scale for Depression (HRSD, [120]), the Global Deterioration Scale (GDS, [255]) and the dementia scale (BDS, [24]) were also administered. The neuropsychological assessment included psychometric tests, qualitative tests [195] and tests derived from the information-processing paradigm [297]. The diagnostic procedure of the MMC has been described in [316].

\section{Follow-up assessment}

The follow-up assessment, which lasted 1.5 to 2 hours, was carried out by research assistants who were trained and supervised by the clinicians working in the MMC with regard to the 
neuropsychiatric and neuropsychological diagnostic procedures. The follow-up assessment consisted of a clinical and a neuropsychological part.

The clinical measures of the follow-up assessment included a medical history, a standardized questionnaire, the MMSE and the HRSD. The questionnaire inquired about the patient's perception of any changes in his or her cognitive functions over the last two years. Four neuropsychological tests were used to evaluate cognitive changes over the period between the first and the second assessments: the auditory verbal learning test (AVLT, [183]), the Revised Trail Making Test (R-TMT; [324]), the Stroop Colour Word Interference Test [183] and the Memory Scanning Task (MST; paper and pencil version [31]). These tests are described extensively in chapter 7 . The neuropsychological tests were selected on the basis of the literature on ageing and dementia that suggest that memory decline and cognitive slowing are among the first aspects of Alzheimer's disease and on previous results from our group concerning patients with age-associated memory impairment $[31,81,124,132,140$, $146,238,299,300,307]$.

\section{Data analysis}

The number of parameters was reduced to three main variables by means of composite scores. These variables were: memory, sensomotoric speed and cognitive speed. Table 1 lists the individual test variables that formed the three composite scores. The memory score was composed of parameters that were thought to represent different aspects of secondary memory. Sensomotoric speed was composed of those test variables that were thought to reflect the basic routine cognitive operations, such as visual scanning and reading. Cognitive speed consisted of variables that were thought to best reflect the speed of mental processing. In order to form the composite scores, individual test scores were converted to standard zscores so that the different tests for each cognitive measure could be combined. For transformation into $z$ scores, the means and the standard deviations of the normative data of different age groups published by Houx (1991) were used as reference. For a comprehensive discussion on the rationale of the composite scores, the reader is referred to Houx (1991).

The composite score of a given variable was regarded as impaired compared to that of the normative data when its value was below the arbitrary level of -2 . In order to obtain a measure of change within the individual patient, we also examined whether the individual performances had declined or improved. A decline of a given variable was defined as a decrease of 2 or more of the composite score of that variable, whereas an improvement was defined as an increase of 2 or more.

Parametric variables, such às age and time needed to perform a given neuropsychological test, were compared between groups by using t-tests for independent samples. In order to make comparisons within groups, t-tests for paired samples were used. Wilcoxon rank sum tests were used for non-parametric variables, Two-tailed analyses were used to assess the significance of differences between groups; a level of $5 \%$ was considered statistically significant. One-way analyses were only used in the analysis of the differences between the patients who developed dementia and those who did not. 
Table 1. Composite cognitive measures and their core variables

\begin{tabular}{ll}
\hline Measures & Core variable \\
\hline Memory & Auditory Verbal Leaming Test \\
& 1. maximum number of words recalled \\
2. total number of words recalled \\
3. total number of words of the delayed recall \\
1. basic speed of memory scanning test "\%"task, time (seconds) \\
2. basic speed of concept-shifting test (TMT-A): time (seconds) \\
3. reading speed of Stroop test (Stroop-1): time (seconds) \\
4. basic speed of memory scanning test, one letter: time (seconds) \\
1. slope of memory scanning test: time in seconds for test with three letters \\
2. Stroop interference (Stroop-III): time (seconds) \\
3. concept-shifting interference (TMT-C): time (seconds)
\end{tabular}

\section{Results}

\section{Description of the patients}

Of the 65 patients who fulfilled the inclusion criteria of the study, 5 had moved from their previous address and could not be traced despite intensive effort. Of the remaining 60 patients, 13 subjects dropped out of the study: 1 had died of a heart attack and 12 declined to participate ( 3 found the tests too tiring, 3 refused because "they no longer had complaints", 1 was unable to come due to personal circumstances and the other 5 gave no reason). Only 1 of the patients who found the test too tiring was considered by her daughter to have deteriorated cognitively, whereas the others were not considered by their relatives to be developing dementia.

The remaining group of 47 patients consisted of 28 men and 19 women. The mean followup period was $25.0 \pm 3.6$ months. The mean age was $57.5 \pm 11.0$ years. Twenty-one patients $(44 \%)$ were between 40 and 55 years, 12 (25\%) between 55 and 65 years old, 14 (29\%) were 65 years or older. Twenty-six patients were classified as stage 2 and 21 as stage 3 of the GDS [255]. Thirty-four (71\%) had an initial MMSE score of 27 or higher, indicating a high global level of cognitive functioning. The initial Hamilton depression scores of 33 subjects were below the cut-off score of 13 that has been proposed as a criterion for Age-Associated Memory Impairment (AAMI [59]), whereas 10 patients had a score between 13 and 18 , and 3 had a depression score higher than 18 . 
Table 2. Means and standard deviations for clinical and cognitive measures

Measure

$\frac{\text { Year0 }}{n \text { mean }(\mathrm{sd})} \quad \frac{\text { Year } 2}{\mathrm{n} \text { mean }(\mathrm{sd})} \underset{(\%)}{\text { Difference }}$ df $\mathrm{P}$

Clinical measures

HDRS score

MMSE score

Cognitive measures

15 Words leaming Test

total recall

delayed recall

delayed recognition

Trail making Test (TMT)

TMT-A

TMT-B

TMT.C

Stroop Color Word Test

Stroop-I (reading)

Stroop-II (color naming)

Stroop-III (interference)

Memory scanning test

baseline ("'o")
with 1 letter
with 2 letters
with 3 letters
with 4 letters

Composite scores

Memory

Sensomotoric speed

Cognitive speed $\begin{array}{lll}46 & 9.9 & (5.7)\end{array}$

$4028.3(1.7)$

$\begin{array}{rrr}45 & 6.5 & (5.5) \\ 45 & 27.5 & (1.9)\end{array}$

$-34$

$43<.001$

$-3$

$37<.01$ 


\section{Effects of attrition}

There were no differences in age, level of education and the initial scores on the MMSE between those patients who were tested in year 2 and those who were not. The only variable that was different between the two groups was the initial score on the HRSD $(9.9 \pm 5.7$ in the patients who were retested, versus $13.3 \pm 5.5$ in those who were not retested: $p<0.05$, Wilcoxon rank sum test, two-tailed). Thus, there was some selective attrition in this study: the patients who could not been retested had a higher level of depressive symptomatology, than with the entire cohort.

\section{Group cbanges}

Table 2 shows the numbers of observations, the means and the standard deviations for the clinical and cognitive measures at the initial assessment and at follow-up for the 47 patients who attended the follow-up assessment. Some data are missing, because a number of patients found the tests to too tiring and wanted to stop prematurely, despite encouragement by the test assistant. As a consequence, the composite score that comprised the variable that was missing could not be obtained. The mean scores on the HRSD and the MMSE of the tested group had decreased significantly (two-tailed t-test for paired samples). None of the single cognitive variables had changed significantly, but the composite score of cognitive speed had increased significantly at follow-up.

Table 3. Development of the composite scores at follow-up, by initial peffomance

\begin{tabular}{|c|c|c|c|c|c|}
\hline \multirow[t]{2}{*}{ composite score } & \multirow{2}{*}{$\begin{array}{l}\text { initial } \\
\text { assessment }\end{array}$} & \multirow[t]{2}{*}{$N(\%)$} & \multicolumn{3}{|c|}{ follow-up assessment } \\
\hline & & & dedined & unchanged & improved \\
\hline \multirow[t]{2}{*}{ Memory $(n=45)$} & impaired: & $36(80)$ & $20(56)$ & $3(8)$ & $13(36)$ \\
\hline & not impaired: & $9(20)$ & $3(33)$ & $4(44)$ & $2(22)$ \\
\hline \multirow[t]{2}{*}{ Sensomotoric speed $(n=41)$} & impaired: & $36(88)$ & $11(31)$ & $14(39)$ & $11(31)$ \\
\hline & not impaired: & $5(12)$ & $1(20)$ & $3(60)$ & $1(20)$ \\
\hline \multirow[t]{2}{*}{ Cognitive speed $(n=36)$} & impaired: & $31(86)$ & $2(6)$ & $15(32)$ & $14(61)$ \\
\hline & not impaired: & $5(14)$ & $1(20)$ & $4(80)$ & $0(\cdot)$ \\
\hline
\end{tabular}

Note: Values represent numbers of patient (\%).

Table 3 shows the number of patients with impaired and unimpaired composite scores at the initial assessment and the development of these scores at follow-up. The memory score was initially impaired in the majority of the patients who were tested. Memory appeared to have deteriorated at follow-up in more than $50 \%$ of these patients. In contrast, most of the patients who initially had impaired scores of sensomotoric and cognitive speed did not appear to have declined further at follow-up. 
Development of dementia

At the time of follow-up, 4 patients were found to have become clearly demented by the two medical specialists, and 1 other patient was diagnosed as questionable dementia (i.e., suspected of having dementia, but some data were missing). One patient was diagnosed as demented by one specialist and as questionable dementia by the other. The other 41 patients were not found to have become demented at follow-up. Thus, the two raters showed $100 \%$ agreement when the two categories of dementia and questionable dementia were combined.

Table 4. Clinical measures and composite scores by diagnosis at follow-up

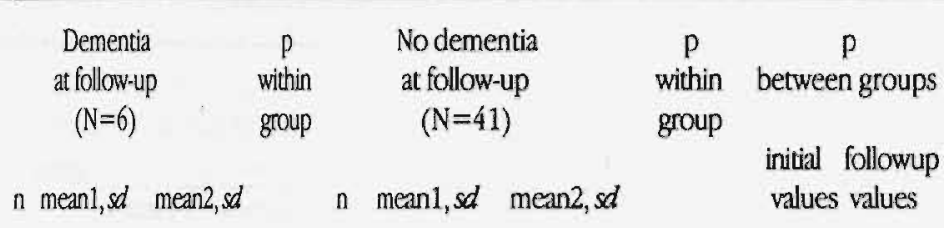

\section{Clinical measures}

\begin{tabular}{|c|c|c|c|c|c|c|c|c|c|}
\hline Initial age (years) & $6 \quad 66.09 .4$ & & & 4155.8 & 10.5 & & \multicolumn{3}{|c|}{$<.05$} \\
\hline HDRS score & $\begin{array}{lll}6 & 9.7 & 3.3\end{array}$ & $6.6 \quad 4.6$ & ns & 409.9 & 5.9 & $6.8 \quad 5.9$ & $<.001$ & ns & ns \\
\hline MMSE score & 528.21 .3 & $27.2 \quad 1.7$ & ns & 3528.4 & 1.8 & 27.51 .9 & $<.01$ & ns & nS \\
\hline \multicolumn{10}{|c|}{ Composite scores } \\
\hline Memory & $6 \cdot-14.86 .9$ & .16 .65 .4 & ns & $41 \cdot 7.5$ & 6.3 & -8.37 .5 & ns & $<.01$ & $<.01$ \\
\hline Sensomotoric speed & d5 $\quad-5.8 \quad 4.6$ & $-13.0 \quad 13.6$ & ns & $\begin{array}{ll}40 & -8.2\end{array}$ & 8.1 & .6 .76 .1 & ns & $\mathrm{ns}$ & $<.05$ \\
\hline Cognitive speed & $3-6.5 \quad 6.8$ & $\begin{array}{rr}-5.6 \quad 6.8\end{array}$ & ns & $37-5.8$ & 4.7 & $-4.6 \quad 3.4$ & $<.05$ & ns & ns \\
\hline
\end{tabular}

$p=$ level of significance. T-test for independent samples, one-tailed, for comparisons between groups; t-test for paried samples;, one-tailed for comparisons within groups.

The 6 patients who were diagnosed as demented (or probably demented) all showed evidence of gradual deterioration in social functioning at follow-up. None of the patients had had cerebrovascular symptoms in the follow-up period, nor any other somatic abnormalities. Therefore, these 6 patients were thought to suffer from probable Alzheimer's disease.

Table 4 shows the characteristics of those patients who became demented in the follow-up period and those who did not. An analysis of the differences between the two groups showed that the patients who developed dementia were on average 10 years older and had significantly worse memory scores, both at the initial and at the follow-up assessment, than the patients who did not develop dementia. In addition, the sensomotoric speed of the patients who developed dementia was significantly worse in the dementing group at follow-up. The two groups did not differ significantly with regard to the initial and the follow-up scores on the HRSD and the MMSE. Within the group of the patients who developed dementia, none of the variables had changed significantly. It should be noted, however, that the number of patients 
who developed dementia was small $(\mathrm{N}=6)$, and that, additionally, several values were missing. Within the group of patients who did not develop dementia, the score on the HRSD had improved significantly, whilst that on the MMSE had decreased. The mean cognitive speed of the non-dementing group had improved significantly.

\section{Relationship between depression and cognition}

Table 5 shows the main characteristics of patients with an initial HRSD score of 13 or more and patients who had a lower depression score. Besides the ratings on the depression scale, no significant differences could be found between the two groups.

Table 5.Clinical measures and composite scores by diagnosis at follow-up

\begin{tabular}{|c|c|c|c|c|c|}
\hline $\begin{array}{l}\text { initial } H R S D \geq 13 \\
\quad(n=13)\end{array}$ & $\begin{array}{c}\mathrm{P} \\
\text { within } \\
\text { group }\end{array}$ & & $\begin{array}{c}\text { initial HRSD }<13 \\
\quad(n=33)\end{array}$ & $\begin{array}{l}p \\
\text { within } \\
\text { group }\end{array}$ & $\begin{array}{c}p \\
\text { between groups }\end{array}$ \\
\hline mean1,sd mean2, sd & & $n$ & mean $1, s d$ mean $2, s d$ & & $\begin{array}{l}\text { initial follow-up } \\
\text { values values }\end{array}$ \\
\hline
\end{tabular}

\section{Clinical measures}

Initial age (yrs)

1359.511 .7

3356.610 .2

HDRS-score

$1316.5 \quad 3.6$

$\begin{array}{lllll}6 & 10.3 & 6.7<.02\end{array}$

$\begin{array}{llll}33 & 7.1 & 3.7\end{array}$

$4.94 .0<.01$

ns

MMSE-score

$\begin{array}{llllll}11 & 28.3 & 1.7 & 26.9 & 1.6 & <.05\end{array}$

$\begin{array}{lll}29 & 28.4 & 1.8\end{array}$

$27.81 .9<.02$

$<.001<.001$

\section{Composite scores}

Memory

$\begin{array}{llllll}12 & -7.8 & 7.2 & -8.9 & 6.9 & \mathrm{~ns}\end{array}$

$32-8.4 \quad 6.5$

$\begin{array}{llll}6.5 & -9.2 & 7.8 & \mathrm{~ns}\end{array}$

nS nS

Sensomotoric speed $11 \quad-5.8 \quad 4.6 \quad-8.2 \quad 7.4 \quad$ ns

$\begin{array}{lllllll}30 & -7.5 & 6.1 & -6.8 & 6.1 & \mathrm{~ns}\end{array}$

Cognitive speed

$\begin{array}{llllll}8 & -6.4 & \text { i } & -5.0 & 2.7 & \text { ns }\end{array}$

$28-5.6 \quad 4.7 \quad-3.8 \quad 3.1<.01$

ns ns

ns ns

ns ns

$\mathrm{p}=$ level of significance. T-test for independent samples, one-tailed, for comparisons between groups; $\mathrm{t}$-test for paired samples, one-tailed for comparisons within groups.

Despite efforts to discontinue psychoactive medication at the initial and the follow-up assessment, 6 patients used psychoactive drugs at the time of the initial assessment: the scores for memory and cognitive speed was somewhat lower in these patients (memory: 12.1 in patients using drugs vs. 9.1 in those without, $p<0.05$ two-tailed Wilcoxon Rank sum test). At follow-up, there was no relationship between the use of psychoactive medication $(n=6)$ and the neuropsychological performance. Within the groups, cognitive speed had improved significantly in the patioents whose initial HRSD scores were below 13 . Twenty-four patients had received a diagnosis of depression (including dysthymia) at the initial assessment and 19 of them also had impaired memory scores. Ten of these 21 patients had deteriorated at followup, but their mean depression score had not changed significantly (HRSD 13.0 6.6 initially, and $12.1 \pm 7.6$ at follow-up). However, the memory scores of 8 of these 21 patients had improved, and so had their depression ratings (initial HRSD score $12,5 \pm 3.3$ and $7.8 \pm 4.6$ at 
follow-up). Of the 6 patients who subsequently developed (clear or questionable) dementia, 3 had received a psychiatric diagnosis at the initial assessment: 1 had been diagnosed as having major depression, 1 as having dysthymia and 1 as having an anxiety disorder. Decliners and nondecliners with depression had memory scores similar to those of patients without depression. There was no significant relationship between the direction of change of the memory scores and that of the depression score.

\section{Discussion}

There are several limitations of the present study that should be considered before discussing the possible implications of its results. First, a substantial number of patients did not come for follow-up: almost $30 \%$ of the original cohort could not be retested. Some patients refused because they found the tests too tiring, others gave no reason for their refusal. The group that could not be reassessed had higher ratings on the HRSD, indicating a higher level of depressive symptomatology. Because a tendency to get stressed and an increased exhaustibility may occur early in the development of dementia [238], the patients who dropped out of the study probably represent a group that is at more risk of becoming demented than those who participated. As a consequence, the results obtained with the retested group probably underestimate the real level of deficits of the entire cohort. Second, quite a few patients could not finish all the neuropsychological tests, especially those tests that demanded relatively more mental effort. For instance, composite scores of cognitive speed could not be obtained in $23 \%$ of the patients who were retested. This is another cause of selective attrition effects in this study, because the patients who could not finish these tests are probably those with the worst performance. Third, a control group was not studied in parallel to the study group. Therefore, the study does not allow for comparisons between the patient group with the general population, with regard to the clinical course of the symptoms studied. Because of these methodological problems, the results of these study should be interpreted with due consideration.

Six out of the 47 patients who were tested (13\%) were thought to have become demented at the two-year follow-up by the two clinicians. Given the fact that the mean age of the population under study was quite low and that the time of follow-up was short, this number is much higher than expected on the basis of known incidence figures. If the same method is applied as has been used by others [227] for estimating the incidence rate in a given group on the basis of the age distribution, the expected number of patients that would develop dementia in two years would be less than one $(0.47)$ in our study population. Thus, the rate observed in this study was much higher than expected. The figures presented here give only some global indication and should be considered with caution, however, because the absolute number of patients was small and we did not study a control group. Nevertheless, these results clearly illustrate the methodological value of services such as the Memory Clinic for research into the very early manifestations of dementia. Furthermore, a follow-up period longer than the two years of our study would probably have detected more cases of dementia, 
as there were several patients who showed a decline of cognitive functions but who were not thought to have become demented.

The present research confirms the findings of other studies that memory decline is among the first signs of Alzheimer's disease $[140,299,300]$. It should be noticed, however, that the patients were referred to a memory clinic, by which the results may be biased by selection. We could not confirm the finding of two other studies that Alzheimer's disease begins with cognitive slowing $[238,307]$. In our study, patients who developed dementia were not cognitively slower than those who did not develop dementia, although the first group had low scores for sensomotoric speed at follow-up. The discrepancy between the results of the two above-mentioned studies and our investigation may be due to the methodological problems as discussed in the previous paragraph, but also to the fact that patients with psychopathological symptomatology had not been excluded from our study. In this respect, our study group was more heterogeneous than that of the other studies. Cognitive slowing has also been found in affective disorders [330] and may therefore not be specific for Alzheimer's disease.

There have been few longitudinal studies of the non-cognitive aspects of early dementia. In fact, patients with psychiatric disorders are usually excluded from most studies. The number of patients who subsequently developed dementia would have been $50 \%$ lower if psychiatric disorders had been an exclusion criterion in our study. Changes of affectivity and personality may be among the earliest features of dementia $[1,172,238,252$, 274]. Therefore, patients with cognitive complaints who also demonstrate psychopathological symptoms may represent a population of particular interest with respect to the early detection of dementia.

The results of the present study do not support the view that the level of depressive symptomatology always parallels the degree of memory impairment. Two patterns of clinical courses could be distinguished: some patients with depression appeared to have deteriorated with regard to the memory score, the HRSD score being unchanged, whilst others showed an improvement of both the depression ratings and the level of cognitive functioning. In the latter group, it is likely that impaired memory is secondary to the depressive symptomatology. In the first group, however, the cognitive functions went on to decline irrespective the level of depression ratings. Therefore, the affective symptoms of this latter group may well be a psychological consequence of cognitive impairments.

Because of the above-mentioned limitations, the findings of the present study cannot be considered as conclusive with regard to the early cognitive changes of Alzheimer's disease. More research still has to be done with larger patient groups and with longer follow-up periods. The study has also shown that neuropsychological tests that are theoretically of interest in the assessment of early dementia may be difficult to administer in practice. Many of the methodological problems that we encountered seem to be inherent to the specific aims of the study, i.e., looking for the first manifestations of dementia. Patients who are the most anxious and uncertain about their cognitive abilities may be the first to refuse to participate. Patients who experience relatively little stress as been a burden, a feature which is probably one of the first characteristics of dementia, may be particularly likely to stop the assessment procedure prematurely. These findings may explain why only a few longitudinal studies into early dementia have been performed so far. They underscore the need for a more 
individualized approach to the diagnosis of early dementia. It may be worthwhile to develop a more qualitative type of assessment as well, in which the reason for failing to finish a given test is also taken into account. The present study will be extended by reassessing the patients after another two years, parallel to a control group of normal subjects, in order to enable comparisons between the patients' course and that of normal subjects. 



\section{A comparison between six current sets of criteria for the diagnosis of vascular dementia*}

\section{Introduction}

Dementia can be caused by a variety of disorders [333], but by far the most frequent causes are Alzheimer's Disease (AD) and vascular dementia (VD) [156]. Therefore, the differentiation between $\mathrm{AD}$ and $\mathrm{VD}$ is of major importance, not only from a clinical viewpoint $[116,178]$, but also for epidemiological research [286]. Several instruments have been developed for this purpose. In 1975, Hachinski and coworkers designed the Ischaemic Score (H-IS) to rate the probability of a vascular pathogenesis in patients with dementia [117]. The H-IS consists of 13 items related to the course, risk factors, signs and symptoms. Although Hachinski's IS has been criticized for several reasons $[70,189,190]$, it appears to differentiate between VD and AD reasonably well, but not between $\mathrm{VD}$ and mixed dementia (i.e., $\mathrm{AD}$ coexisting with cerebral ischaemic lesions) $[79,214,269,327]$. The H-IS has had considerable impact and still serves as the gold standard in clinical research.

Since the publication of Hachinski's IS, at least five other sets of criteria have been proposed to improve the accuracy of the diagnosis of VD (see also the appendix of this chapter). Rosen's modification of Hachinski's IS (R-IS) was formulated on the basis of pathological data from 14 patients. In this version, five of the original H-IS items were omitted that did not contribute to the clinical differentiation between AD and VD [269]. Loeb and Gandolfo validated the H-IS by using CT scan data from 101 patients with dementia and proposed another version of the IS (L-IS) on the basis of their findings. The L-IS includes only

"Verhey FRJ, Lodder J, Rozendaal N, Jolles J. Submitted for publication 
four of the original H-IS items, and is expanded to include single- or multiple low density areas on CT scan $[192,193]$. The criteria for Vascular Dementia from the DSM-III-R, similar to those from the DSM-III [7], comprise, besides the presence of dementia, presence of a stepwise deteriorating course with a patchy distribution of deficits; focal neurological signs and focal neurological symptoms; and evidence from history, physical examination or laboratory tests of significant cerebrovascular disease judged to be etiologically related to the disturbance. The DSM criteria are not quite unambiguous. The "patchy pattern of deficits" is difficult to operationalize, and it is not clear whether the second criterion should be taken literally as signs and symptoms, or, more liberally, as signs and/or symptoms, as has been interpreted by others [93]. Erkinjuntti et al. defined VD, multi-infarct type, as dementia evolving in connection with acute neurological symptoms or signs and/or findings on CT indicating multiple cortical and/or deep vascular lesions of the brain $[80,83]$. Finally, a recent proposal for the diagnosis of VD was presented in a report by the State of California Alzheimer's Disease Diagnostic and Treatment Centers (ADDTC) [46]. In this proposal, no reference is made to any course characteristic; neuroimaging evidence of an infarct (by CT or MRI) is necessary; and at least two ischaemic strokes are required, or, in case of one stroke, the evidence of a temporal relationship to the onset of dementia. Both Erkinjuntii's criteria and those from the ADDTC allow for a diagnosis of possible $\mathrm{VD}$, when there is a single stroke without a temporal relationship to dementia. Moreover, the ADDTC criteria classify Binswanger's syndrome also as possible VD.

Thus far, none of the above sets of criteria for VD has clearly been proven to be superior and all are currently used for clinical and research goals. In this study we applied the six sets to a sample of demented patients visiting an university hospital, in order to compare the prevalence rates of VD and $\mathrm{AD}$ as detected by each criterion and to obtain insight into the similarities and differences between the different criteria. The aim of this study was to examine whether or not the different criteria can be regarded as interchangeable, which would have implications for the comparison of studies into VD or $\mathrm{AD}$ carried out with different criteria.

\section{Patients}

The data of the patients consecutively referred to the Maastricht Memory Clinic (MMC) were used for this study. Included were patients with completed diagnostic work-up, and with dementia according to the DSM-III-R criteria[7] whithout any somatic, neurological or psychiatric disorders other than of primary degenerative and/or vascular origin. The diagnostic procedure has been described elsewhere [316] and includes a semi-structured history provided by the patient and his or her caregiver, a standardised psychiatric interview, an internal, neurological examination and neuropsychological investigation, laboratory tests and a CT-scan of the brain (in most cases: Philips Tomoscan 310). A neurologist experienced in cerebrovascular pathology (JL) examined all CT scans for the presence of ischaemic (lacunar or cortical) infarctions and leukoaraiosis, according to regular criteria[80] and without knowledge 
of the clinical diagnosis. Only CT scans made during the assessment of dementia, and not those eventually made in the acute stage of a stroke, were used for this study.

\section{Methods}

Soon after the diagnostic work-up, all relevant clinical data of each patient had been entered in the database of the MMC. These included, among others, all items from the Hachinski's Ischaemic scale, number, type and localization of the strokes, relevant CT scan data, existence of a temporal relationship with the onset of dementia and clinical features of binswager's syndrome as defined in the ADDTC report.[46] Thus, although most of the patients had been assessed before the ADDTC criteria were published (March 1992), the different sets of criteria could be applied in retrospect for all selected patients. The only data that were not available pertained to the DSM-II-R criterion for VD of a "patchy distribution of deficits". This aspect was ignored, because it was felt that it could not be operationalized easily. Furthermore, the second criterion of the DSM-III-R was interpreted as signs and/or symptoms. Cut-off scores for $\mathrm{VD}$ and $\mathrm{AD}$ were used as described in the original publications. For uniformity of terms, the concepts Multi-Infarct Dementia as used by the different authors [7, 83, 117, 192, 269] and probable IVD [46] are all referred to as "Vascular Dementia". Likewise, the term "mixed dementia" denominates patients with IS scores between the cut-off values, probable and haemodynamic type $\mathrm{VD}[80,83]$ and possible Ischaemic VD [46]. A fourth category is formed by the patients who could neither be classified as $\mathrm{AD}$ nor as $\mathrm{VD}$ or mixed dementia. Frequencies of the diagnosis of VD obtained for a specific set of criteria were compared pairswise with those for $\mathrm{AD}$ (or mixed dementia) obtained for the corresponding set of criteria to exclude a vascular etiology (see also table 2)

The agreement between the different sets of criteria was assessed by calculating the kappa statistics. Kappa is the rate of observed agreement between a single pair of sets adjusted for the proportion of the agreement that can be exspected to occur by chance.

\section{Results}

One hundred and nine patients were included in this study. The mean age of the patients ( 52 male, 57 female) was $70.1 \pm 8.8$ years. The patients were mildly or moderately demented, as reflected by a mean score on the Mini-Mental State Examination [97] of $17.9 \pm 5.8$. The main clinical and CT-scan characteristics are summarized in Table 1. 
Table 1. Patient characteristics $(\mathrm{N}=109)$

\begin{tabular}{|c|c|c|}
\hline Feature & $\mathbf{N}$ & (\%) \\
\hline Acute onset & 24 & $(19 \%)$ \\
\hline Stepwise deterioration & 25 & (20\%) \\
\hline Fluctuating course & 26 & $(21 \%)$ \\
\hline Noctumal confusion & 20 & $(16 \%)$ \\
\hline Preserved personality & 54 & $(44 \%)$ \\
\hline Depression & 41 & $(33 \%)$ \\
\hline Somatic complaints & 30 & $(24 \%)$ \\
\hline Emotional lability & 27 & $(22 \%)$ \\
\hline (History of) hypertension & 29 & $(23 \%)$ \\
\hline History of TIA's or stroke(s) & 31 & (28\%) \\
\hline Signs of associated atherosclerosis & 24 & (19\%) \\
\hline Focal neurological symptoms & 19 & $(15 \%)$ \\
\hline Focal neurological signs & 36 & $(29 \%)$ \\
\hline Multiple low dense areas on CT & 11 & (9\%) \\
\hline Single low density area on CT & 13 & $(10 \%)$ \\
\hline Evidence of mulciple strokes & 13 & $(10 \%)$ \\
\hline with temporal relation to dementia & 13 & $(10 \%)$ \\
\hline without temporal relation to dementia & - & $\cdot$ \\
\hline Evidence of a single stroke & 24 & $(22 \%)$ \\
\hline with temporal relation to dementia & 10 & $(9 \%)$ \\
\hline without temporal relation to dementia & 14 & $(13 \%)$ \\
\hline Binswanger's syndrome & 3 & $(2 \%)$ \\
\hline
\end{tabular}

\section{Agreements and discrepancies between the criteria}

Table 2 shows the numbers of patients diagnosed according to each criterion. Thirty-nine had vascular dementia according to at least one set of criteria, whereas only 8 patients were diagnosed as VD by all sets of criteria. Seventy-five patients had $\mathrm{AD}$ diagnosed by at least one set of criteria, whereas 51 patients were diagnosed as such by all sets. The R.IS and the H-IS resulted in the highest number of patients with VD (about 30\%), whereas only $13 \%$ of the subjects were diagnosed as having VD when the ADDTC, the DSM or Erkinjuntti's criteria were used. The proportion of patients diagnosed as $\mathrm{AD}$ varied from $64 \%$ (using the L-IS) to $54 \%$ (using the DSM criteria). Between 8 and $32 \%$ of the patients were diagnosed as suffering from mixed dementia. The proportion of patients who could not be classified as either VD, mixed dementia or $\mathrm{AD}$, ranged from $32 \%$ (using the DSM criteria) to $2 \%$ (using the H-IS). 
Table 2. Frequencies of vascular dementia and Alzheimer's disease according to six different sets of criteria

\begin{tabular}{|c|c|c|c|}
\hline Criterion & · & $\mathrm{N}$ & $(\%)$ \\
\hline \multicolumn{4}{|c|}{ Hachinski's Ischaemic Scale } \\
\hline VD: & $\mathrm{H} \cdot \mathrm{IS}$ score $\geq 7$ & 32 & $(29)$ \\
\hline mixed: & H-IS score $>4$ and $<7$ & 10 & (9) \\
\hline $\mathrm{AD}:$ & NINCDS/ADRDA criteria, and H-IS score $\leq 4$ & 65 & $(60)$ \\
\hline unclassified & & 2 & (2) \\
\hline \multicolumn{4}{|c|}{ Rosen's Ischaemic scale } \\
\hline VD: & R-IS score $\geq 4$ & 36 & (33) \\
\hline mixed: & R.IS score of 3 & 6 & (6) \\
\hline$A D:$ & NINCDS/ADRDA criteria, and R-IS score $\leq 2$ & 64 & (59) \\
\hline unclassified & & 3 & (3) \\
\hline \multicolumn{4}{|c|}{ Loeb's ischaemic Scale } \\
\hline $\mathrm{VD}$ : & L.IS score $\geq 5$ & 23 & (21) \\
\hline mixed: & L-IS score of 3 or 4 & 12 & (11) \\
\hline $\mathrm{AD}:$ & NINCDS/ADRDA criteria, and L-IS score $\leq 2$ & 70 & (64) \\
\hline unclassified & & 4 & (4) \\
\hline \multicolumn{4}{|l|}{ DSM-III(-R) } \\
\hline VD: & 'Multi-infarct dementia' & 15 & (14) \\
\hline mixed: & (not covered by DSM criteria) & - & - \\
\hline $\mathrm{AD}:$ & 'Primary Degenerative Dementia' & 59 & (54) \\
\hline unclassified & & 35 & (32) \\
\hline \multicolumn{4}{|c|}{ Erkinjuntti's criteria } \\
\hline $\mathrm{VD}:$ & Multi-infarct dementia (MID) & 14 & (13) \\
\hline mixed: & 'PVD' and 'Haemodynamic cype demencia' & 26 & (24) \\
\hline $\mathrm{AD}:$ & NINCDS-ADRDA criteria, with exclusion of MID & 61 & $(56)$ \\
\hline unclassified & & 8 & (7) \\
\hline \multicolumn{4}{|c|}{ ADDTC criteria } \\
\hline W: & 'Probable IVD' & 15 & (13) \\
\hline mixed: & 'Possible IVD' & 9 & (8) \\
\hline AD: & NINCDS-ADRDA criteria, with exclusion of probable or possible IVD & 66 & $(61)$ \\
\hline unclassified & & 19 & (17) \\
\hline
\end{tabular}

Note: $\mathrm{VD}=$ Vascular Dementia; $\mathrm{AD}=$ Alzheimer's Disease; H-IS = Hachinski's Ischaemic Score [119]; R-IS = Rosen's Ischaemic Score [269]; L-IS = Loeb's Ischaemic Score [192]; MID = Multi-Infarct Dementia; PVD = Probable Vascular dementia [83]; IVD = Ischaemic Vascular Dementia|46]; unclassified $=$ not classifyable as either $\mathrm{VD}, \mathrm{AD}$ or mixed dementia

In Tables $3 a$ and $3 b$, the kappa values between all possible combinations of criteria are shown for $V D$ and $A D$ (using the different criteria to exclude vascular dementia), respectively. On the average, the agreement for $A D$ was somewhat higher than for $\mathrm{VD}$ (kappa: 0.75 versus 0.70 ), whereas only fair agreement existed for mixed dementia (kappa: 0.32). Concerning the diagnosis of $\mathrm{VD}$, almost perfect agreement existed between the ADDTC criteria and those of 
Erkinjuntti and the DSM, and between the H-IS on the one hand and the R-IS and the L-IS on the other. Moderate agreement existed between the H-IS and R-IS on the one hand and the ADDTC and Erkinjuntti's criteria on the other. With respect to the diagnosis of $\mathrm{AD}$, the H-IS, the R-IS and the L-IS showed considerable agreement, and so did the ADDTC with the DSM-III-R and Loeb's criteria.

Table 3a. Agreement (kappa-values) berween the different sets of criteria for the diagnosis of vascular dementia.

\begin{tabular}{lccccc}
\hline Criteria & Hachinski & Rosen & Loeb & DSM-Ill & Erkinjunti \\
\hline Rosen & 0.894 & & & & \\
Loeb & 0.808 & 0.777 & & & \\
DSM-III & 0.648 & 0.622 & 0.722 & & \\
Erkinjuntti & 0.559 & 0.536 & 0.744 & 0.687 & \\
ADDTC & 0.588 & 0.564 & 0.772 & 0.867 & 0.937 \\
\hline
\end{tabular}

Table 3b. Agreement (kappa-values) between the different sets of criteria to exclude vascular dementia for the diagnosis of Alzheimer's Disease.

\begin{tabular}{lccccc}
\hline Criteria & Hacbinski & Rosen & Loeb & DSM-III & Erkinjunti \\
\hline Rosen & 0.905 & & & & \\
Loeb & 0.825 & 0.807 & & & \\
DSM-III & 0.664 & 0.684 & 0.681 & & \\
Erkinjuntii & 0.775 & 0.794 & 0.829 & 0.555 & \\
ADDTC & 0.675 & 0.657 & 0.805 & 0.869 & 0.680 \\
\hline
\end{tabular}

Note: Kappa statistics, interpretation of the level of agreement: 0.0 to 0.2 , slight; 0.2 to 0.4 , aar; 0.4 to 0.6 , moderate; 0.6 to 0.8 , substantial; and 0.8 to 1.0 , almost perfect [177].

\section{Reasons for diagnostic discrepancies}

The highest disagreement occurred between the H-IS and the R-IS on the one hand and the ADDTC and Erkinjuntti's criteria on the other: 32 patients had a H-IS score indicative of VD, but no more than 20 of them would not have been diagnosed as such according to the ADDTC criteria (21 according to Erkinjuntti's criteria). Five of these patients had an insidious onset of dementia and no clinical history of stroke or hypodense areas on the CT scan, and would have been diagnosed as $\mathrm{AD}$ by the NINCDS/ADRDA criteria when the ADDTC criteria were used for the exclusion of VD; however, four of them demonstrated slight unexplained focal signs and / or symptoms (e.g., one-sided palmomental reflex), whereas the fifth patient demonstrated a fluctuating course and scored on all one-point items of the H-IS, leading to the high H-IS 
score. Nine other patients with VD according to the H-IS were classified as mixed dementia by the ADDTC: three patients with Binswanger's syndrome (as defined in the ADDTC report) and six with evidence of only one stroke without a clear temporal connection with dementia. Six patients identified with the H-IS as VD could not be classified appropriately by the ADDTC criteria: 3 patients were said to have become demented acutely instead of insidiously (thus not fulfilling criteria for $A D$ ), neither was there evidence of stroke in the history, physical examination or on CT scan (thus not fulfilling the criteria for possible or probable VD). Moreover, three other patients became demented as a result of a single stroke without there being demonstrable hypodense areas on the CT scan. The ADDTC criteria do not cover these two situations, but Erkinjuntti's criteria would have classified them as mixed dementia.

Of the 24 patients who had a history of one single stroke, in 14 there was no temporal relationship between the stroke and the onset of dementia. Most of these patients had had a minor stroke many years before the first manifestations of cognitive impairment. With the R-IS, all these 14 patients would have been diagnosed as VD, whereas with the H-IS and the L-IS 11 and 10 patients would have had this diagnosis respectively. In contrast, only 3 of the 14 patients, namely those who showed evidence of more than one infarct on the CT scan, had been diagnosed as VD by the ADDTC or Erkinjuntti's criteria, whereas the other 11 were merely diagnosed as mixed dementia.

\section{Discussion}

The present study shows that application of the six sets of criteria for the diagnosis (or the exclusion) of vascular dementia led to differences in the frequency of $\mathrm{AD}$ and $\mathrm{VD}$ that cannot be neglected. For example, the IS of Rosen resulted in more than twice as many patients with VD than the DSM-III-R criteria. The ADDTC and Erkinjuntti's criteria showed almost perfect agreement with each other, and so did the ischaemic scales of Hachinski and of Rosen. Otherwise, the sets of criteria cannot be regarded as interchangeable. The choice of a particular set of criteria appears more critical in demonstrating a vascular etiology for the diagnosis of $\mathrm{VD}$ than in making such an etiology unlikely for the diagnosis $\mathrm{AD}$. In the 'clear-cut' patients, e.g., in those who demonstrated clear evidence of multiple strokes in their history, clinical examination, and CT'scan, different criteria led to similar diagnoses. The criteria diverged when information from one category did not confirm the other, e.g., evidence of stroke from CT scan without focal neurological symptoms or vice versa.

In the absence of neuropathological data, no definite conclusion can be drawn as to which of the criteria is superior. However, pathological data are of relative value for the confirmation of the clinical diagnosis of VD, since the existence of ischaemic infarctions at autopsy does not prove that they actually produce dementia. Only the ADDTC and Erkinjuntti's criteria provide the opportunity to assess explicitly the issue of causality between strokes and dementia by making use of the criterion of a temporal relationship with dementia. This is also true for the DSM criteria ("evidence of cerebrovascular disease judged to be related to dementia"), although rather implicilly. The present study shows that if the aspect of a temporal connection 
is taken into account, it influences the diagnostic outcome considerably. The same is true when the neuroimaging data are taken into consideration (the L-IS, the ADDTC and Erkinjuntti's criteria). E.g., evidence of ischaemic infarcts on CT scan was found in our study in 45 to $100 \%$ of the patients diagnosed as VD, and in 0 to $13 \%$ of the patients with $\mathrm{AD}$, depending on which criterion was used. Ischaemic infarcts have been described in other studies in 20 to $90 \%$ of the patients with VD and in 0 to $37 \%$ of the patients with $A D[80,250$, 263,291 ]. These great variations are likely to be explained by differences in the criteria used for the diagnosis of VD, as has been suggested by others [286].

In summary, this investigation shows that the results of studies which use different criteria for the diagnosis or the exclusion of VD must be compared with caution. Given the impact of the issues of temporal connection and of neuroradiological data, the ADDTC and Erkinjuntii's criteria can presently be regarded as the criteria of choice, although they still await further validation by pathological studies. The controversy on whether VD is overdiagnosed [33] or underdiagnosed [228] may be related to differences in the criteria used, which is possibly an overlooked source of interstudy outcome variance. 


\section{Depression, insight and personality changes in Alzheimer's disease and vascular dementia*}

\section{Introduction}

Alzheimer's disease $(\mathrm{AD})$ and vascular dementia (VD) are the two most common causes of dementia [156]. Differentiation between these two disorders in an early phase is important as remedial therapeutic interventions for vascular dementia are probably then more successful [116]. Furthermore, homogeneous diagnostic groups are a prerequisite for epidemiological and pharmacological research. In the past, many attempts have been made to diagnose VD on clinical grounds. Mayer-Gross et al.[199] described, in the Handbook of Clinical Psychiatry $(1969)$, its typical clinical presentation as follows:

"The patient with VD is in the first stages of the disease restlessness, very emotional, and inclined to wander at night. (..) Menory and intellectual impairment may be preceded by a caricature of one or more personality traits....) Drive and initiative diminished (...) yet judgement and the basic personality may be well preserved and the patient can retain remarkably good insight ...leading to despondency and pessimism.(..) Depression is noticeable at some stage in almost a third of cases".

This description provided Hachinski and coworkers with the basis for the ischemic scale (IS) as a tool to assess the cerebrovascular etiology of dementia [119]. Hachinski's IS is still widely used and contains, among others, the items 'depression' and 'relatively retained personality'.

In view of the widespread popularity of Hachinski's IS, it is apparently assumed that depression and preserved personality occur more frequently in VD than in AD. Additionally, a

\footnotetext{
"Verhey ERT, Ponds RHWM, Rozendaal N and Jolles J,submitted for publication
} 
relatively intact insight into ones' deficit is also generally regarded as being more characteristic for patients with VD than for patients with $\mathrm{AD}[7,199]$. However, there is little empirical evidence that $\mathrm{AD}$ and $\mathrm{VD}$ can be discerned reliably on the basis of these features. Hardly any study has determined whether an intact insight and a preserved personality can be used to differentiate between $\mathrm{AD}$ and VD. The few studies that there are on the relationship between depression and the type of dementia have yielded conflicting results. For instance, depression has been found to occur more often in VD than in $\mathrm{AD}$ [64], less often in VD than in $\mathrm{AD}$ [252], or in similar frequencies [94]. It is relevant to note that these studies for the most part included inpatients and that the results are thus difficult to generalize to less severely demented outpatients. Thus, reliable data on the prevalence of clinical characteristics that differentiate between $\mathrm{AD}$ and $\mathrm{VD}$ in relatively mild stages of dementia are virtually non existent. Additionally, variations in the frequencies of occurence of a given feature seem most related to differences in the diagnostic criteria. For instance, some have used Hachinski's IS to distinguish between $\mathrm{AD}$ and $\mathrm{VD}[64,65]$. When depression and personality are studied, the use of this scale leads to circularity since it includes these two features. Another source of variation may stem from differences in the study samples. For example, some have reported that depressive symptoms occur more often in the early stages of $\mathrm{AD}[35]$ and in female subjects $[35,182]$. Moreover, the age of onset of dementia may be a possible important characteristic that influences the results of comparisons of different groups [78]. Thus, the severity of dementia, sex distribution, and age of onset are potentially confounding factors that should be taken into account when comparing the prevalence of depression in different groups of demented patients.

The present study addresses the question whether patients with VD and patients with $\mathrm{AD}$ differ in the prevalence of depression, lack of insight, and personality changes. In order to rule out differences attributable to differences in related sample characteristics, we compared patients with $\mathrm{AD}$ to patients with VD who were carefully matched for age, sex, and extent of dementia. The aim of this study was to examine whether or not these clinical features would help to differentiate between $\mathrm{AD}$ and $\mathrm{VD}$ in a relatively early phase of the disease.

\section{Patients and methods}

We selected demented outpatients with $\mathrm{AD}$ and VD who had been consecutively referred to the Maastricht Memory Clinic of the University Hospital of Maastricht, The Netherlands [316]. Dementia was diagnosed according to DSM-III-R criteria. [7] AD was diagnosed using the NINCDS/ADRDA criteria for possible and probable Alzheimer's Disease [207]. The diagnosis of VD was based on the DSM-III-R criteria for dementia and a score of 4 or more on Rosen's modified ischemic score [269]. In contrast to Hachinski's original IS, the modified IS does not include the items depression and retained personality. The severity of dementia was assessed by the Global Deterioration Scale (GDS) [255]. The degree of functional impairment was measured by the dementia scale of Blessed-Tomlinson-Roth (BDS) [24]. 
The original group consisted of 93 patients with $\mathrm{AD}$ and 48 patients with VD. Compared to the patients with VD, the patients with $\mathrm{AD}$ were significantly younger (74.0 vs. 70.7 years, $p<.05$ ) and less severely demented, as measured with the GDS (mean GDS score 4.8 and 4.3 respectively; Wilcoxon test, $d f=1, p<0.02$ ). Therefore, we selected 48 patients from the $A D$ group who were matched pairswise with regard to the GDS score, age, and sex to the 48 patients with VD. Matching was preferred rather than controlling and correcting for an effect of the GDS score and age by means of an ANCOVA procedure, in order to avoid the problems of using an ordinal variable as a covariate [285]. Matching was carried out by a research assistant who was blind to patient data other than these three characteristics. Forty-four out of the 48 patients were matched within the same GDS score. Patients were matched for age within \pm 3 years, all patients were matched for sex.

A distinction was made between depression, as a syndrome, and depressive symptoms. Depression was diagnosed according to the DSM-III-R criteria for a major depressive episode, ignoring the organic exclusion [7]. Depressive symptoms were assessed using Hamiltons' rating scale for depression, which also served as a global measure for the extent of the depressive symptomatology [120]. The HRSD has been proven to be reliable in demented patients [113].

For the purpose of this study, impaired awareness or lack of insight was defined, according to Schachter (1990), as the absence of knowledge or recognition of cognitive deficits. In analogy to others, the extent of awareness was assessed by comparing the patients' and the caregivers' history $[205,278]$. Awareness of deficits was rated on a four-point rating scale, ranging from 4 ('intact') to 1 ('absent'), using the following guidelines (see also appendix of chapter 11). At the initial interview, the patient was asked to say why he or she had been referred. The caregiver had been asked not to make any comments before being requested to do so. Awareness was judged to be intact (awareness score, $A S=4$ ) when the cognitive problems were mentioned spontaneously by the patient in reply to the opening question: of why he or she had been referred, and when the history of the caregiver corresponded with that of the patient. Awareness was scored as mildly impaired $(\mathrm{AS}=3)$ when the patient commented spontaneously about his or her memory in reply to the opening question, but when there were apparent discrepancies between the patient's and the caregiver's anamnesis. Awareness was scored severely impaired $(A S=2)$ when there the patient made no comment to the first question, when he acknowledged memory impairments only on explicit questioning, and when there were clear discrepancies between the patient's and the caregiver's anamnesis. Awareness was scored as absent $(\mathrm{AS}=1)$ when the patient denied any problems related to dementia, even when asked explicitly. Awareness was scored by the neuropsychiatrist, who saw the patient first. In order to assess inter-observer reliability, awareness was scored independently by two clinicians (a neuropsychiatrist and a neuropsychologist) in a subgroup of 20 consecutive patients. The kappa value was 0.70 , indicating substantial interobserver agreement [177]. Details about the assessment of awareness have been described elsewhere [321].

As an indication of the extent of the change in personality, the sum of the scores for the BDS subitems concerning changes in personality was calculated (BDS personality subscale, 
items 12-19). These eight subitems were: increased rigidity, increased egocentricity, impairment of regard for feelings of others, corsening of affect, impaired emotional control, e.g. irritability; hilarity in inappropriate situations, diminished emotional responsiveness, and sexual misdemeanour, appearing de novo in old age. Each subitem could be scored as 1 (the specific change in personality was present for more than 6 months, or as 0 (no change, or the change was present for less than 6 months). Scoring was carried out by an experienced neuropsychiatrist on the basis of the information provided by the caregiver

\section{Statistical analysis}

A t-test was used to compare both groups on the variable age. Because of the ordinal level of most of the data (GDS, HDRS, BDS, and the awareness score) nonparametric tests were used: Wilcoxon two-sample tests, analysis of variance (ANOVA) with rank sums, and Spearman's rankorder correlation coefficients (Rs) [285]. Probability ( $P$ ) values lower than 0.05 were regarded as being significant.

\section{Results}

The main patient characteristics of both groups are shown in Table 1. Seventy-nine percent of the patients had (very) mild or moderate dementia, as measured by the GDS score (very mild, GDS 3: $4 \mathrm{AD}, 5 \mathrm{VD}$; mild, GDS 4: 12 patient in each group; moderate, GDS 5:22 AD, 23 VD; severe, GDS 6: $10 \mathrm{AD}, 8 \mathrm{VD}$; very severe, GDS 7: no patients). Only nine patients, three with $\mathrm{AD}$ and six with VD, fulfilled DSM-III-R criteria for major depression. These numbers were too small to carry out a meaningfull statistical analysis.

Table 1. Patient characteristics of matched groups

\begin{tabular}{llll}
\hline & $\begin{array}{c}\text { Alzheimer"s disease } \\
n=48\end{array}$ & $\begin{array}{c}\text { vascular dementia } \\
\mathrm{n}=48\end{array}$ & $\mathrm{P}$ \\
\hline Age (mean, sd) & $72.9 \pm 7.6$ & $73.9 \pm 7.7$ & $\mathrm{~ns}$ \\
Sex $(\mathrm{M} / \mathrm{F})$ & $31 / 17$ & $31 / 17$ & \\
GDS (mean, sd) & $4.8 \pm 0.9$ & $4.7 \pm 0.9$ & $\mathrm{~ns}$ \\
BDS (mean, sd) & $7.9 \pm 4.2$ & $8.0 \pm 4.5$ & $\mathrm{~ns}$ \\
Dumation in years (mean, sd) & $3.28 \pm 2.4$ & $3.31 \pm 2.1$ & $\mathrm{~ns}$ \\
Education $(1-7)$ & $3.6 \pm 1.3$ & $3.7 \pm 1.5$ & ns \\
\hline
\end{tabular}

Note: GDS = Global Deterioration Scale, BDS = Blessed Dementia Scale. Level of education according to [314]: 1 : primary school - 7: University grade. 


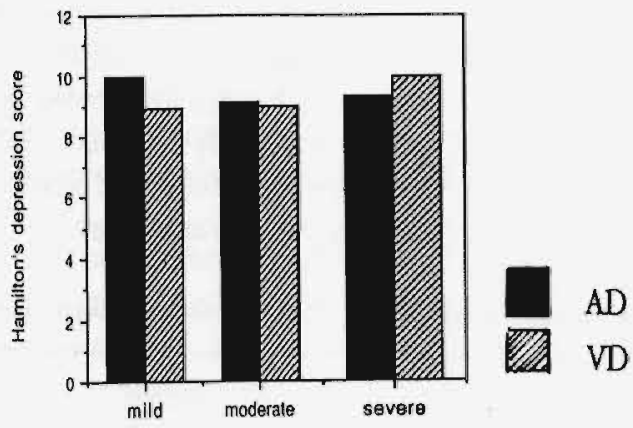

Figure 1. Mean Hamilton's depression scores in patients with Azheimer's disease and vascular dementia, by severity of dementia

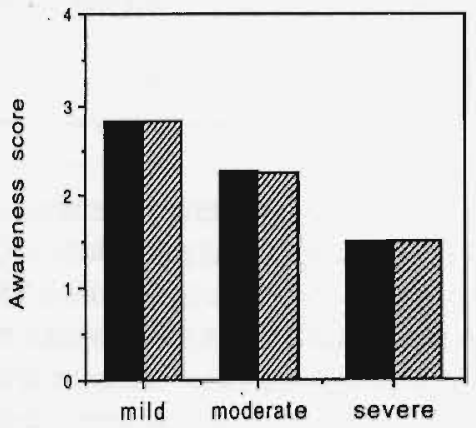

Figure 2. Mean awareness score in patients with Alzheimer's disease and vascular dementia, by severity of dementia

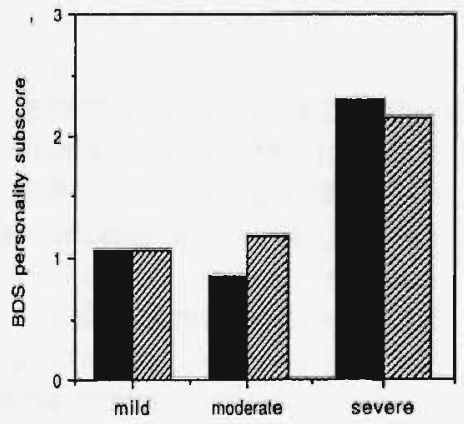

Figure 3. Mean BDS personality-subscore in patients with Alzheimer's disease and vascular dementia, by severity of dementia 
The mean scores on the HRSD did not differ significantly between the patients with $\mathrm{AD}$ and those with VD $(9.4 \pm 9.4$ for patients with $\mathrm{AD}$, and $9.1 \pm 4.5$ for patients with $\mathrm{VD} ; \mathrm{Z}=-0,16$, Wilcoxon test, n.s.). An analysis of the separate items of the HRSD did not reveal differences in any of the subitems between the groups. A correlation between the severity of dementia (GDS score) and the score on the HRSD was virtually nonexistent in both groups (Rs: 0.04 and 0.01 for $\mathrm{AD}$ and $\mathrm{VD}$, respectively, n.s.), i.e., mildly demented patients of both etiologies had similar depression scores compared to the more advanced stages.

Table 2. Distribution of awareness scores among patients with Alzheimer's disease and vascular dementia

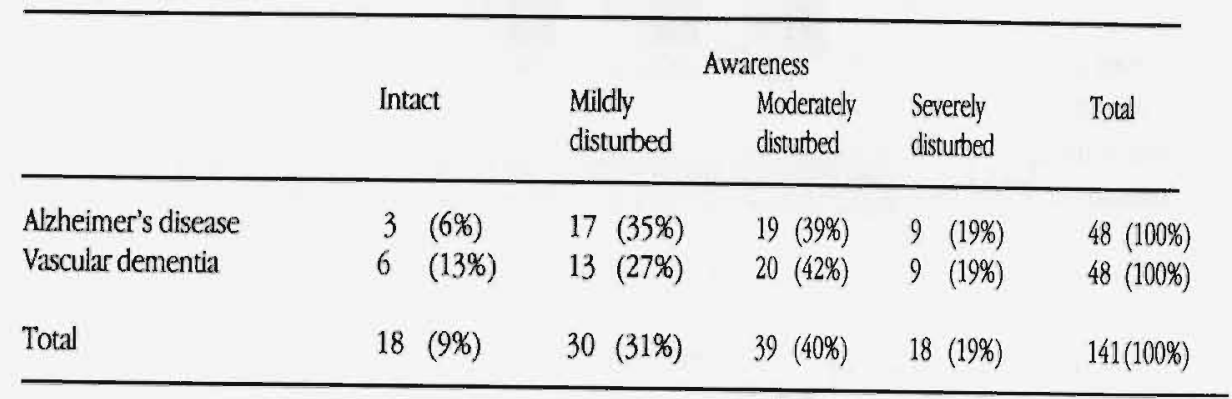

The distribution of awareness scores is shown in table 2. The awareness scores were more or less equally distributed among patients with $\mathrm{AD}$ and patients with VD (Wilcoxon test, n.s.). The mean score on the personality subscale was $1.9 \pm 1.2$ for patients with $A D$ and $1.3 \pm 0.8$ for patients with VD. The scores were not statistically significantly different (Wilcoxon test).

The number of patients with depression, the Hamilton depression scores, the awareness scores and the personality scores did not differ significantly between patients with $A D$ or VD when the items were analysed separately with a rank-sum ANOVA for mild (GDS 3 and 4), moderate (GDS 5), and severe dementia (GDS 6) (see figures).

\section{Discussion}

The main conclusion that emerged from this study is that level of depression, lack of insight, and personality changes occurin $\mathrm{AD}$ and in VD with comparable frequencies.

Differences between $A D$ and $V D$ with regard to depressive symptoms have been examined in four other studies $[64,65,94,252]$. The patients from our study were all ambulatory, in contrast to the patients from these previous studies, which included hospitalized patients from specialized wards. Therefore, the results of our study are difficult to compare with those of the other reports. The two studies that used the IS of Hachinski $[64,65]$ yielded higher depression scores in the VD patients than in the $\mathrm{AD}$ patients. However, this might be related to the fact that patients with depression have an increased change of being diagnosed as having VD when the IS of Hachinski is used. The severity of dementia of the different groups 
was mentioned only in two studies, and in both reports the patients with $\mathrm{AD}$ appeared on average significantly more severely demented than the patients with VD. In one of these two studies [94], the patients with $\mathrm{AD}$ had higher depression scores than the patient with VD, whereas the other study reported the opposite [64]. The study sample showing higher depression scores in patients with VD consisted of chronic inpatients who were, on average, nine years older than the patients of the other study. These differences probably influence the study outcome.

There are hardly any other studies that explicitly addressed differences in the level of awareness between these two types of dementia. We know of only one study, in which awareness was reported to be significantly less impaired in $\mathrm{VD}$ than in $\mathrm{AD}$ [65]. However, this study cannot be regarded as conclusive because there were no clear diagnostic criteria and because of the vague operationalization of the terms.

Depression and preserved personality have been examined implicitly in several studies that investigated the validity of Hachinski's IS $[79,83,93,192,214,269]$. Without exception, neither depression nor preserved personality appeared to discriminate between $\mathrm{AD}$ or $\mathrm{VD}$. Thus, there is no basis for the view that depression, preserved awareness, and/or personality favor a vascular etiology rather than Alzheimer's disease. Therefore, these features should not be included in the diagnostic criteria for vascular dementia.

Both the awareness scores and the BDS-personality subscores were associated with the degree of the dementia, irrespective its etiology (Rs: 0.56 , with $p<.0001$ and Rs: 0.23 with $p<0.05$, respectively). In addition, age correlated significantly with the GDS score (Rs $=0.49$, with $p<0.0001$ ). When we would have compared the original group of (younger and less disturbed) patients with $\mathrm{AD}$ to the patients with $\mathrm{VD}$, the level of awareness and the personality changes would have been significantly different between $\mathrm{AD}$ and $\mathrm{VD}$. However, this finding should be regarded as spurious, as the two groups would not have been comparable with regard to age and severity of the dementia. This underscores the necessity to control for these factors when different types of dementia are compared.

A limitation of our study may be that no pathological data were available to confirm the clinical diagnosis. However, it should be emphasized that pathological data are of relatively little value for the confirmation of the clinical diagnosis of VD, since the existence of ischemic infarctions at autopsy does not prove that they actually caused the dementia. In this study, the diagnosis of VD was based in this study on Rosen's IS. More elaborated diagnostic criteria have been published very recently, but these were not available at the time of this study. Although patients in the vaguest category of 'mixed' dementia (i.e., with a score on Rosen's IS of 3) were not included in this study to avoid diagnostic uncertainties, the results may be still somewhat different if the new and probably more accurate criteria would have been applied.

\section{Conclusion}

We conclude that depression or depressive symptoms, a lack of insight into ones' deficits and/or a preserved personality are not of relevance for the differential diagnosis between $\mathrm{AD}$ and $\mathrm{VD}$ in outpatients with relatively mild stages of dementia. 



\section{Dementia, awareness and depression*}

\section{Introduction}

It is well established that symptoms of depression occur more frequently in patients with Alzheimer's disease $(\mathrm{AD})$ and other dementing disorders than in the normal elderly population $[94,182,202,340]$. Insight into the relationship between depression and dementia contributes to the understanding of the pathogenesis of the disorder and has important implications for the choice of therapeutic strategies. An overlap in the neuropathological and neurochemical substrate of depression and $\mathrm{AD}$, cerebrovascular disease and Parkinson's disease has been suggested as a biological explanation for the higher prevalence rate of depression in dementia (e.g., $[62,295,342,343])$. In contrast, psychological mechanisms underlying the depressive symptoms of demented patients have received much less attention.

In this respect, the phenomenon of awareness in dementia may be relevant. The terms unawareness of deficits, lack of insight and anosognosia (which are used interchangeably in this article, in accordance with the literature) refer to a lack of knowledge or recognition of one's deficits (e.g., [205] ). The few publications that exist on anosognosia in dementia report a general pattern of decreasing insight with increasing severity of the dementing process $[87$, 205, 258]. Depressive symptoms have been reported to occur also more frequently in mild dementia than in the more severe stages $[64,94]$. Thus, depressed feelings in demented patients may be seen as an understandable reaction, as a patient becomes aware of his or her loss of intellectual capacity and impending deterioration; as insight deteriorates with the progression of dementia, the patient may be less liable to suffer from depression (e.g., [118, $323,333])$.

\footnotetext{
*Verhey FRJ, Rozendaal N, Ponds R W H M, Jolles]. Intemational Joumal of Geriarric Psychiarry 1993; 8: in press
} 
The aim of this study was to ascertain whether or not depressive symptoms are indeed more likely to occur in demented patients who have some degree of awareness of their deficits than in those who lack this insight. We expected the outcome to show that patients who are relatively aware of their deficits are more likely to develop depression or, at least, depressive symptoms and that anosognosia may 'protect' against depressive symptoms in dementia.

\section{Patients and methods}

The subjects were 170 outpatients with dementia who had been consecutively referred to the Maastricht Memory Clinic of the University Hospital of Maastricht [316]. Dementia was diagnosed according to the DSM-III-R criteria [7]. Patients were diagnosed as possible or probable Alzheimer's Disease (AD) based on the NINCDS criteria [207]. The diagnosis of vascular dementia (VD) was based on a score of 4 or more on Rosen's modified Ischaemic Score [269]. It should be noted that, in contrast to Hachinski's original IS, the modified IS does not include the items depression and retained personality. Other etiologies were diagnosed according to regular criteria. The severity of dementia was assessed by the Global Deterioration Scale (GDS) [255]. In the assessment of depression in dementia, a distinction was made between the syndrome of depression and symptoms of depression. A diagnosis of a depressive syndrome was made according to the DSM-III-R criteria for Major Depressive Episode without applying the organic exclusion [7]. The Hamilton Depression Rating Scale of Depression (HRSD, 17 item version [120]) was administered to obtain a measure of the severity of depression and as a checklist for depressive symptoms. The reliability of this clinician-rated scale has been demonstrated in demented patients $[113,235]$.

\section{Assessment of awareness}

No generally accepted standardized assessment procedure to measure anosognosia or impaired awareness is available to date. For the purpose of this study, impaired awareness was defined, according to Schachter (1990), as the absence of knowledge or recognition of cognitive deficits. In analogy to others, the degree of awareness was assessed by comparing the patients' and the caregivers' history (see [205, 278]). Awareness of deficits was rated on a four-point scale, ranging from 4 ('intact') to 1 ('absent'), using the guidelines as are shown in the appendix of this chapter. It was scored by the neuropsychiatrist who carried out the initial interview with the patient and his or her caregiver.

\section{Statistical analyses}

Because of the ordinal level of most of the data, non-parametric tests were used for the analysis of the dependent variables of awareness score, GDS score and depression score: rankorder correlation coefficient (Spearman) for correlations between variables, Wilcoxon's test 
(rank sums) for group differences, and Kruskal-Wallis rank ANOVA with covariates, which approximates a chi-square distribution [285]. T-tests were used to examine differences in age between the groups. Probabilities greater than 0.05 were considered as non-significant. In the analysis of the HRSD-subitems, a level of P less than 0.002 was adopted according to a Bonferroni-type adjustment, to protect against spurious significance arising from multiple comparisons.

\section{Results}

The study group consisted of 170 patients, 103 with $\mathrm{AD}$ and 43 with VD and 24 with other causes (seven Parkinson's disease, four frontal lobe dementia, two normal pressure hydrocephalus, two alcohol related dementia, two Huntington's disease and seven miscellaneous causes). Their mean age was $71.2 \pm 8.6$ years, sex ratio 77 males $/ 94$ females. The mean duration of cognitive symptoms was $38.3 \pm 28.2$ months. Most patients were mildly or moderately demented, as measured by the GDS score (very mildly demented, GDS 3: 17 patients, mildly demented, GDS 4: 77 patients; moderately demented, GDS 5: 55 patients; severely demented, GDS 6: 21 patients). The mean score on the Mini-mental State Examination [97] was $18.1 \pm 6.1(2-29)$. The three groups (AD, VD and others) differed with regard to age $(70.7,74.0$ and 68.0 years respectively, $\mathrm{p}<.05)$ and the degree of dementia (average GDS scores 4.4, 4.7 and 4.1 respectively, Wilcoxon chi-square:11.3, $\mathrm{DF}=2, \mathrm{p}<0.05$ ).

Table 1. Distribution of awareness scores by severity of cognitive decline (GDS score) among 170 patients with dementia.

\begin{tabular}{|c|c|c|c|c|c|}
\hline \multirow[b]{2}{*}{ GDS stage } & \multicolumn{5}{|c|}{ Awareness-score } \\
\hline & $\begin{array}{l}4 \\
\text { Intact }\end{array}$ & $\begin{array}{l}3 \\
\text { Mildly } \\
\text { disturbed }\end{array}$ & $\begin{array}{l}2 \\
\text { Moderately } \\
\text { disturbed }\end{array}$ & $\begin{array}{l}1 \\
\text { Severely } \\
\text { disturbed }\end{array}$ & Total \\
\hline 3 Very mild dementia & $5 \quad(29 \%)$ & $11(65 \%)$ & $1 \quad(6 \%)$ & $=(0 \%)$ & $17(100 \%)$ \\
\hline 4 Mild dementia & $5(7 \%)$ & $45(58 \%)$ & $17(22 \%)$ & $10(13 \%)$ & $93(100 x)$ \\
\hline 5 Moderate dementia & $2(4 \%)$ & $16(29 \%)$ & $30 \quad(55 \%)$ & $7 \quad(13 \%)$ & $48(100 \%)$ \\
\hline 6 Severe dementia & . $\quad(0 \%)$ & $1(5 \%)$ & $7 \quad(33 \%)$ & $13(62 \%)$ & $41(100 \%)$ \\
\hline
\end{tabular}

In order to assess inter-observer reliability, awareness was scored independently by two clinicians (a neuropsychiatrist and a neuropsychologist) in a subgroup of 20 consecutive patients. The kappa value was 0.70 , indicating substantial interobserver agreement $[177]$.

Lack of insight was a common phenomenon in this population. Of the 170 patients, only twelve (7\%) were able to give their history spontaneously without there being clear 
discrepancies between their histories and that given by the caregiver. On the other hand, only 30 patients (18\%) showed a complete lack of insight. Patients with $\mathrm{AD}$ tended to have somewhat higher awareness scores than patients with $\mathrm{VD}$, but this difference was not statistically significant (Kruskal-Wallis, chisq $=2.62, \mathrm{df}=2$ ).

Table 2. Correlation coefficients (Spearman rank order, Rs) between awareness-scores and score on the Hamilton Rating Scale of Depression (HRSD) in demented patients $(n=169)$.

\begin{tabular}{|c|c|c|}
\hline & mean & Rs \\
\hline HRSD, total score: & 9.14 & 0.03 \\
\hline \multicolumn{3}{|l|}{ Individual HRSD items: } \\
\hline 1 Depressive mood & 1.17 & 0.14 \\
\hline 2 Suicidal ideation & 0.05 & 0.04 \\
\hline 3 Feeling of guilt & 0.26 & 0.12 \\
\hline 4 Insomnia, early & 0.09 & 0.07 \\
\hline 5 Insomnia, middle & 0.13 & -0.05 \\
\hline 6 Insomnia, late & 0.16 & 0.01 \\
\hline 7 Work and activities & 1.99 & -0.19 \\
\hline 8 Retardation & 1.03 & -0.09 \\
\hline 9 Agitation & 0.71 & -0.04 \\
\hline 10 Anxiety, psychic & 1.08 & $0.27^{*}$ \\
\hline 11 Anxiety, somatic & 0.57 & 0.11 \\
\hline 12 Gastrointestinal complaints & 0.20 & 0.02 \\
\hline 13 Somatic complaints, general & 0.65 & 0.06 \\
\hline 14 Genital symptoms & 0.10 & 0.10 \\
\hline 15 Hypochondriasis & 0.05 & 0.01 \\
\hline 16 loss of weight & 0.26 & -0.04 \\
\hline 17 Psychological insight & 0.53 & -0.05 \\
\hline
\end{tabular}

Spearman-rank order correlation, ${ }^{*} p<0.0001$

The awareness scores correlated significantly with age (Spearman rank order correlations -Rs:0.33 with $p<0.0001$ ). The distribution of the awareness scores by the severity of dementia (measured by the GDS) is shown in Table 1. There was a significant negative correlation between the GDS score and the awareness score (Rs: 0.51 , with $p<0.0001$ ). In contrast to what has been found in other studies [64,94], we did not find a clear correlation berween the HRSD score and the GDS scores (Rs: 0.11 , n.s.), i.e., patients who were mildly demented had similar depression-scores to those with severe dementia. Only 16 out of 169 patients (9\%), 9 with $\mathrm{AD}, 4$ with VD, and 3 with other causes, fulfilled the DSM-III-R criteria for major depression (missing data: $n=1$ ). The distribution of the awareness scores was not unequal between the patients with depression and those without (Kruskal-Wallis, chisq. $=.05, \mathrm{df}=1$, 
ns). Actually, the majority of the patients with depression ( 9 out of 16 ) displayed little or no insight into their cognitive deterioration. Using rank ANOVA with age and GDS scores as covariate, no significant relation could be found between the awareness score and the depression score ( $\mathrm{F}=0.42, \mathrm{df}=3, \mathrm{~ns}$ ). Spearman rank order correlation coefficients between the awareness scores and the scores on the HRSD (total and subitems) are shown in table 2. The mean score on the HDRS was $9.1 \pm 4.9(0-25)$. No significant correlation could be found between the total HRSD score and the awareness score ( $R s=-0.03, n . s$.). As is shown in this table, only the score on the item 'psychic anxiety' showed a weak but significant correlation with the awareness scores, i.e., patients showing a greater degree of insight had higher scores on this subitem $(R=0.28$ with $P<0.0001)$.

\section{Discussion}

This study showed that impaired awareness was very common in this sample of demented patients. In only 7 percent of the patients were there no obvious discrepancies between the history provided by the patient and that of the caregiver. However, it was also noted that about half of the patients spontaneously reported problems with their memory at the opening question, indicating at least some partial knowledge of their impairments. Thus, it seem oversimplified to state that "it is not possible for patients with memory disorders to remember that one has forgotten" [95].

A statistically significant correlation was found between the awareness scores and severity of the dementia syndrome as measured by the GDS score, a finding which is consistent with other reports $[87,258]$. No significant relationship could be demonstrated between the level of awareness and the presence of major depression or the total score on the HRSD. The relationship between awareness and depression in dementia has only been studied previously in one study [87], in which also no significant correlation could be found between denial scores and Hamilton depression scores. An additional finding in our study was that the depression scores did not correlate with the severity of the dementia. Therefore, this study cannot provide any support for the view that depression lessens as awareness deteriorates with the increasing severity of dementia. A relatively intact awareness was not clearly associated with the development of depressive symptoms in dementia and anosognosia did not protect against depression. A modest but significant correlation could be found between awareness scores and the HRSD-subscores on the item 'psychic anxiety' even after a Bonferroni type of adjustment. Although this finding should be considered with caution untill further research has been done, it may suggest that feelings of anxiety may be secondary to insight into cognitive decline. This supports the notion that symptoms reflecting an internal psychic state are related to psychological mechanisms [182]. In contrast, major depression in dementia, apparently unrelated to the degree of insight, may be predominantly based on neuropathological or neurochemical abnormalities. The possible implications for the treatment of these complaints, namely a more psychological approach to symptoms of anxiety, and an emphasis on biological interventions for the treatment of major depression, should be looked at in further studies. 
It could be argued that the absence of a relationship between awareness and depression is due to the fact that impaired awareness pertains to both cognition and depression. In this view, a low score on HRSD items might simply reflect anosognosia for depressive feelings, rather than the absence of these symptoms. However, this does not seem very likely, since one may expect that severity of dementia would have correlated then with the depression scores, which is not the case. Additionally, the applicability of the HRSD as an objective clinical rating scale, in contrast to self-report measures, has been demonstrated even in advanced dementia $[113,235]$.

Some limitations in this study have to be considered. It is likely that patients who experience cognitive decline will consent more readily to further evaluation in the Memory Clinic than those without any complaints, and thus some degree of selection bias may have been occurred. Furthermore, a four-point rating scale may be too global for the assessment of such a complex phenomenon as awareness of deficits. Systematic investigation of anosognosia has begun only recently, and to date, there is no assessment tool available that provides reliable and valid information with certainty. Finally, the assessment of awareness in this study was based on discrepancies between the patients' and the caregivers' reports and thus depends largely on the reliability of the caregiver. However, caregivers of patients with $\mathrm{AD}$ were recently found to be reliable informants of their relatives' memory deficits [168] and thus, this does not seem an important source of error.

\section{Conclusions}

Our findings suggest that a relatively intact level of awareness in demented patients is not accompanied by an increase in the prevalence of depression. However, it may lead to some specific depressive symptoms, notably psychic anxiety. More research is needed to confirm theresults of this study. 


\section{Summary and concluding remarks}

This thesis deals with clinical aspects of the early diagnosis and the differential diagnosis of dementia, particularly in relation to depression and normal forgetfulness. The thesis opens with a general introduction on the rationale of an early diagnosis of dementia (Chapter 1). The patients of the studies described have been examined in the Memory Clinic of the Academic Hospital of Maastricht, between May 1986 and October 1992. The aims, methods and organisation of the Maastricht Memory Clinic is presented. The MMC addresses especially patients with cognitive impairments which are not severe enough to merit the diagnosis of dementia or for patients who are suffering from mild stages of dementia. This specific aim of the MMC is reflected by the characteristics of the patients: they were on average younger and less cognitively impaired than in traditional psychogeriatric departments. Therefore, the results of the studies of this thesis cannot be generalized to populations of older, more severely disturbed patient groups without due consideration.

An introductory review of the literature on mild dementia is presented in Chapter 2. The aim of this review is to present the state-of-the art knowledge and to identify critical issues in the research of mild stages of dementia. Dementia is generally regarded as a syndrome, not as a disease, but important differences exist in the interpretation of this notion. Therefore, the various meanings of the term are discussed first. Next, the most frequently used criteria for the clinical diagnosis of dementia (the DSM-III-R criteria) and of Alzheimer's disease (those of the NINCDS/ADRDA workgroup) are amply discussed. The accuracy of the diagnosis of dementia has largely been improved by these diagnostic criteria. An attempt is made to depict the clinical features of Alzheimer's disease before it has developed to frank dementia, on the base of empirical data from longitudinal studies. The earliest manifestations of Alzheimer's disease are qualitative rather than quantitative: patients may perform on traditional 
neuropsychological tests within normal limits, but they need more time and get tired easily. Non-cognitive features are prominent in early stages: feelings of anxiety and psychological vulnerability, passivity, an increased dependency on the environment and decreased spontaneity are likely characteristcs of very early Alzheimer's disease. Unfortunately, noncognitive aspects have received much less attention than cognitive aspects. Among the earliest neuropsychological deficits are impaired delayed recall, fluency and general speed of information processing. It is concluded that the diagnosis of early Azheimer's disease is based on clinical judgement and repeated cognitive measures over time. It is not known, however, which features can best be followed, and how long the intervals between the tests should be. In recent decades, many different proposals have been made for the classification of the state of mild memory impairment. These are discussed in view of their reliability and predictive validity. Important clinical issues in the assessment of mild dementia are the differentiation from normal aging, age-associated memory impairments and depression, clinical variations related to subtypes of dementia, and the influence of comorbidity and age upon the diagnosis of dementia.

The issues of studies of this thesis can be divided into three main themes: the influence of the discipline that make the diagnosis, clinical features of non-demented subjects with cognitive complaints and clinical characteristics of patients with dementia.

\section{The influence of the discipline that make the diagnosis}

In view of the fact that the diagnosis of dementia still depends to a large extent on clinical judgement, it is quite remarkable that the influence of different specialists upon this diagnosis has so far been neglected. Therefore, an experimental study of interdisciplinary differences in the diagnosis of dementia was carried out, described in Chapter 3. An inquiry was held among the participants of the Dutch consensus meeting on dementia. Eighty-five clinicians diagnosed the case descriptions of ten patients with various forms of cognitive dysfunctions. There was no difference between the disciplines with regard to syndrome diagnoses. However, with regard to aetiology, significant differences existed between the specialties: neurologists were more often in agreement with a "golden standard" diagnosis than the psychiatrists. Furthermore, the level of consensus increased with the severity of the cognitive disturbances of the patients. The diagnosis was more often erroneous when depressive symptoms existed. The nature of the diagnoses appeared to depend on the discipline of the diagnostician. Finally, the number of the various terms that were used by the responding clinicians was inversely related to the severity of the cognitive deterioration.

In Chapter 4, the discrepancies in diagnostic outcome were examined between the multidisciplinary, criteria-based approach in patients referred to the Memory Clinic and the monodisciplinary approach of the referrers. Of the 278 patients not fulfilling the criteria dementia, 19 had been previously diagnosed as being demented (specificity 0.93). In 60 of 152 demented patients, dementia had not been diagnosed before (sensitivity 0.61 ). Underreporting was frequent in mildly demented patients and in patients with coexisting 
depressive symptoms. In patients referred by psychiatrists, sensitivity rates for dementia and Alzheimer's disease were low; in patients referred by neurologists, depression often went unreported.

In order to investigate whether the use of the MMC diagnosis as the 'gold standard' in the above-mentioned study was correct, a study was carried out on the predictive validity of the diagnoses of dementia from the MMC. Available clinical data were collected on the course of the cognitive and behavioral symptoms of 114 patients, 81 with Alzheimer's disease and 33 with vascular dementia. Most patients were only mildly or moderately demented at the initial assessment. After a mean period of 41 months (16.7-71.2), $95 \%$ of the patients were described as having deteriorated since the initial assessment. This figure indicates that the diagnosis of dementia, even in relatively mild stages, can be made reliably. This finding supports the validity of the diagnostic model of dementia, as used in the MMC.

Mild dementia is not a diagnosis that can be made by rudimentary bedside screening instruments, but neither do we believe that the diagnosis can only be made by esoteric experts that use highly sophisticated technology. The main difference between our approach and the usual monodisciplinary approach is the combined and integrated use of common assessment methods that are used in daily clinical practice, and the consequent application of generally accepted diagnostic criteria.Many sorts of data have to be taken into account. For instance, it was found that referrers quite often failed to investigate the functional significance of the cognitive impairments, as is required by criterion C of the DSM-III-R.

The studies further confirm the stereotype ideas about several disciplines: psychiatrists and psychologists are good at recognizing depression, neurologists look especially at brain diseases, whereas physicians from nursing-homes and geriatricians pay particular attention to potentially treatable causes such as polypharmacy. These are all important aspects, but unfortunately a price has to be paid for specialization; psychiatrists did not always complete the somatic diagnostic work-up, and neurologists and other somatically orientated clinicians did not recognize psychiatric disorders. Thus, all of the specialties had specific advantages, but also certain flaws. This is not say that each discipline should be blamed for not being an expert in their colleague's area of interest, but rather to plead for more modesty when working separately, as no one specialism knows everything. Neurologists, psychiatrists and neuropsychologists complement each other in the early stages of dementia, whereas internists or geriatricians may be of value in the older patient who has several other severe medical disorders and significant comorbidity.

Thus, a more systematic use of multidisciplinary services for patients with cognitive disorders is to be encouraged. However, such a multidisciplinary approach should be more than the mere sum of the different elements. Special emphasis must be laid upon the integrative abilities of each one: the different disciplines should be familiar with each other's terminology and paradigms. Trainees in one relevant area need to be better educated in the integration of their specific findings with those of others. 


\section{Clinical aspects of non-demented patients with cognitive complaints}

It is generally known that memory and other cognitive complaints are seen in a variety of organic disorders, one of which is Alzheimer's disease. However, it is often not sufficiently recognized that cognitive impairments are also a common feature of many functional psychiatric disorders: in clinical practice major depression is often seen as the single functional cause. The study described in Chapter 6 was carried out in order to determine whether psychiatric conditions other than depression are relevant in elderly patients with memory disturbances. Therefore, 430 consecutive outpatients (242 males, 188 females; mean age 61.7 years) who visited the Maastricht memory clinic were examined by using a standardized diagnostic procedure which included somatic, neurological, psychiatric and neuropsychological examinations. Psychiatric disorders were diagnosed according to DSM-III(-R) criteria. Of 152 patients with dementia, 34 had a secondary depressive syndrome and 19 another secondary psychiatric disorder. Of the 37 patients with an organic mental disorder other than dementia, 16 had an organic mood disorder. Of the other 241 patients, 152 had 159 primary psychiatric diagnoses: there were 100 diagnoses of mood disorders (depression and dysthymia) and 59 diagnoses of other psychiatric disorders, especially adjustment disorders, anxiety disorders and personality disorders. Together, no fewer than $40 \%$ of all primary or secondary psychiatric diagnoses concerned other disorders than mood disorders. Therefore, the differential diagnosis of memory deficits should encompass more than just looking at whether depression is present or not. Moreover, the relationship between psychiatric disorders and cognitive functioning is complex and requires a careful psychiatric assessment. The studies of this thesis illustrate the idea that a too linear view on causality between the two, i.e, depression causing cognitive impairments, does not do justice to the complex interplay between cognition, substrate, emotionality and subjective meaning.

From a scientific point of view, studying the combination of psychiatric symptoms and cognitive deficits in the elderly is of importance, because they may be the very first features of dementing disorders. Longitudinal studies have indicated that these symptoms are often too mild to merit the diagnosis of major depression. This condition may be covered more-or-less by other DSM diagnoses, notably dysthymia. Cbapter 7 reports a study in which the nature of cognitive functioning was examined in a group of 25 middle-aged dysthymic subjects, who sought help for memory complaints in the Maastricht Memory Clinic. There were no differences in the performance of primary memory. However, their performance for secondary memory was significantly inferior to that of controls matched for age, sex-distribution and educational level. In addition, the performance on a memory scanning task (with subtasks with increasing memory load) and the Stroop Colour-Word Interference Task was also impaired in dysthymic subjects. The results suggest that dysthymic patients are characterised by a problem with effort demanding processes; the patients appear to make more use of a controlled processing strategy, which takes them more time and energy and which results in subjective complaints.

Recent neuropsychological concepts have made it possible to unravel the process of retaining information into different psychological operations, each one of which can be 
analysed separately, e.g., attention, speed of processing, susceptibility for interfering stimuli, encoding strategies and rehearsal strategies, and automatic versus controlled processing of information. 'Tasks derived from this paradigm may provide more insight into the psychological processes of early Alzheimer's disease than the tasks that are usually adhered to in medical practice, in which old-fashioned terms such as short-term and long-term memory still dominate. The findings of the study described in chapter 7 illustrate this point clearly: all dysthymic patients studied had scores within the normal range on Folstein's Mini-Mental State Examination, a widely used, but static and not very demanding test for non-demented subjects. In contrast, when there was increasing memory load, the performances of the patients diverged significantly from those of the controls. The questions whether the cognitive dysfunctions found in the memory clinic population can be generalised to all dysthymic subjects, cannot be answered in the present study. Interestingly, the profiles of the dysthymic patients had much in common with those seen in ageing. It is unknown yet to what extent the neuropsychological deficits of the patients of this study may be caused by a primary degenerative brain disease: we must wauit for the results of a longitudinal study that is presently carried out in our department. As our studies have been carried out in a memory clinic, the relatively high proportion of patients that had cognitive complaints together with functional disorders may be due to our method of patient selection. Therefore, the study into the cognitive aspects of dysthymia should be reproduced in a general psychiatric practice, in order to find out whether these findings can be generalized to less well-selected patient samples.

Longitudinal studies can shed light upon the development of the psychiatric and cognitive symptoms and their mutual relationship, and, hopefully, provide important clues about the prodromes of dementia. In Cbapter 8, a twoyear follow-up study of 65 non-demented patients complaining of their memory is described. Forty seven patients could be reexamined after a mean follow-up period of $25.0 \pm 4$ months. Patients who could be retested had lower initial depression scores than those who were not retested, indicating some selective attrition. Six out of 47 patients were found to be demented at follow-up. This figure is a manyfold of the one that was to be expected. Patients with subsequent dementia were older and performed worse with regard to memory aspects, but were comparable to those who were not demented at follow-up with regard to the depression score, and ratings of cognitive and sensomotor speed. The level of depressive symptomatology did not always parallel the degree of cognitive impairments. Some patients appeared to be improved on both measures of cognition and depression, but others deteriorated with regard to cognitive functions while the depression score remained unchanged. These results indicate that affective disorders may cause cognitive dysfunctions in some patients, and, inversely, be the (psychological) consequence in others. The conclusions of the study described are limited because of the many methodological problems that were involved. For instance, selective attrition seem inherent to this type of study, as those subjects who are the most uncertain and anxious about becoming demented are the most likely to refrain from participating in the study. Larger samples with longer followup periods are necessary. Despite the limitations of this study, it can be concluded that 
departments such as the memory clinic are suitable for clinical research into the earliest features of Alzheimer's disease.

Apart from a possible relationship between the cognitive aspects of psychiatric disorders and dementia, there are other reasons why cognitive functioning deserves more attention than they receive at present. Cognitive aspects of functional disorders are too often viewed as mere epiphenomena, only of diagnostic significance because they are included in some of the DSM-III-(R) criteria. It is important to note that the patients that were examined in our memory clinic sought help primarily for their cognitive problems and not for their psychiatric condition. Cognitive impairments may compromise the individual's capacity to function in demanding activities. Besides, patients may perceive their limited cognitive capacity as the first signs of dementia and become severely distressed. Therefore, the development of therapeutic psychological interventions aimed at coping strategies for the cognitive impairment is another negelcted area of research. Memory clinics can also be useful in this respect.

\section{Clinical aspects of patients with dementia}

The differentiation between Alzheimer's disease and vascular dementia was, untill recently, mainly clinical: Ischaemic scores, in which several clinical features are collected, have long been used as a diagnostic tool for the diagnosis of vascular dementia. However, more elaborate diagnostic criteria have been published recently. The new criteria differ from the old criteria in that brain-imaging data are taken into consideration and in that attention is paid to the temporal relationship between stroke and dementia. Introduction of new criteria for vascular dementia also has an impact on the diagnosis of Alzheimer's disease, as the frequency of this latter diagnosis is directly to the exclusion of other disorders: vascular dementia is the most frequent disorder to be ruled out. Chapter 9 describes a comparison between six currently used sets of clinical criteria for the diagnosis of vascular dementia in a sample of 109 demented patients. These are: the ischaemic scales of Hachinski, Rosen and Loeb, the criteria from the DSM-III, those outlined by Erkinjuntii and coworkers, and those from the State of California Alzheimer's Disease Diagnostic and Treatment Centres (ADDTC). Depending on which criteria were used, the frequency of vascular dementia ranged from $13 \%$ 10 $33 \%$, and the frequency of Alzheimer's disease from 54\% to 64\%. The Ischaemic Scales of Hachinski and Rosen resulted in the highest frequencies of vascular dementia, whereas the criteria of Erkinjuntti et al, the DSM-III criteria and the ADDTC yielded the lowest. The number of patients with vascular dementia was reduced substantially when neuroradiological data and the temporal relationship between stroke and dementia were taken into consideration. The ADDTC criteria and those of Erkinjuntti seem to be the most advisable. The NINCDS-ADRDA criteria for Alzheimer's disease should be adopted on this point, once the validity for the criteria of vascular dementia has been established. Differences in the criteria for vascular dementia and Azheimer's disease may be an overlooked source of interstudy variance. 
The study described in chapter 10 investigated the popular notion that depression, intact awareness or preserved personality favour a diagnosis of vascular dementia rather than Alzheimer's disease. Most of the studies on this subject have been carried out with severely demented inpatients, and confounding factors such as age, sex and severity of dementia have not been taken into account. We compared 48 patients with relatively mild vascular dementia with 48 patients with Alzheimer's disease who were matched for age, sex and severity of dementia. The two groups did not differ with regard to the prevalence of major depression, the mean depression score, the awareness score or the sum of scores on the items of the Blessed dementia Scale concerning personality changes. We concluded that depression, lack of insight and personality changes do not favour an aetiology of vascular dementia rather than Alzheimer's disease.

Chapter 11 reports a study into the relationship between insight into ones' own deficits and depression in demented patients. It is commonly thought that demented patients may become depressed when they become aware of their cognitive limitations, although the empirical evidence for this notion is little. We examined 170 outpatients, 103 with Alzheimer's disease, 43 with vascular dementia and 24 with various other causes, in order to investigate whether or not depressive symptoms were more likely to occur in dementia patients who had some degree of awareness of their cognitive deterioration. Awareness was rated on a four point scale that assessed discrepancies between the patient's and the caregiver's history. The level of awareness was significantly related to the severity of dementia, but not to depression or to the score on Hamilton's depression scale. However, the score on the item 'psychic anxiety' showed a significant correlation with the level of insight. The occurence of anxiety and other non-affective psychiatric symptoms in dementia have received much less attention than depression. It would be worthwhile investigating symptoms of anxiety in demented patients more extensively, as the relationship to insight into one's condition may suggest a more psychological origin. Related to this, symptoms of anxiety may respond better to psychological treatments, such as psycho-education.

The studies in chapter 10 and 11 illustrate an important methodological point as well, namely the necessity of considering age and degree of dementia when the characteristics of the different types of dementia are to be compared. Our patients with Alzheimer's disease differed from those with vascular dementia with regard to the degree of awareness and of personality changes, but these differences disappeared after correction for age and severity of the dementia. This point has not had enough coverage in the literature so far.

In general, cognitive aspects of dementia have received much more attention than the noncognitive aspects, although it is these aspects that cause the caregivers more problems for than those related to cognition. That non-cognitive aspects of dementia are so often neglected may be related to the fact that they are more difficult to measure. Therefore, more refined instruments should be developed in order to establish these non-cognitive aspects more precisely, both qualitatively and quantitatively. Psychopathological symptoms in organic brain disorders are the subject of an ongoing research project in our department. Until better measures for behavioural and emotional symptoms become available, we must rely on the subtleties of clinical evaluation. 



\section{Samenvatting}

Dit proefschrift gaat over de klinische aspecten van de vroege - en differentiële diagnose van dementie. Het proefschrift begint met een algemene beschouwing over het nut van een vroege diagnose bij dementie (boofdstuk 1). De patienten die voor dit proefschrift werden onderzocht, werden tussen mei 1986 en oktober 1992 gezien in de polikliniek voor geheugenstoornissen van het Academisch Ziekenhuis Maastricht. De doelstellingen, werkwijze en organisatie van deze Maastrichtse geheugen-polikliniek worden in dit hoofdstuk beschreven. Deze polikliniek richt zich vooral op patiënten met lichte dementie, of met dermate lichte cognitieve stoornissen dat ze niet voldoen aan de diagnose dementie. Dit weerspiegelt zich in de kenmerken van de patiënten: deze zijn in het algemeen jonger en minder ernstig cognitief gestoord dan in traditionele psychogeriatrische afdelingen. Het generaliseren van de bevindingen van dit proefschrift naar de situatie bij oudere patiènten met ernstiger cognitieve stoornissen dient daarom met enige terughoudendheid plaats te vinden.

Ter inleiding wordt in boofdstuk 2 cen overzicht van de literatuur over het stellen van de diagnose dementie, vooral in een licht stadium, gegeven. Het doel van dit overzicht is de huidige stand van zaken te beschrijven, en belangrijke aspecten voor het onderzoek te bespreken. In het algemeen wordt dementie beschouwd als een klinisch syndroom, maar deze opvatting blijkt in de dagelijkse praktijk verschillend te worden geinterpreteerd. Daarom wordt eerst stilgestaan bij de verschillende betekenissen van het begrip dementie. In het hoofdstuk wordt vervolgens ingegaan op de meest gehanteerde diagnostische criteria voor dementie (de DSM-III-R-criteria) en de ziekte van Alzheimer (de NINCDS/ADRDA-criteria). Door deze criteria is de betrouwbarheid van de klinische diagnose dementie de laatste jaren aanmerkelijk verbeterd. Daarna wordt er een poging gedaan om de allereerste verschijnselen van de ziekte van Alzheimer te beschrijven aan de hand van empirische gegevens uit 
longitudinaal onderzoek. De allereerste verschijnselen van de ziekte van Alzheimer zjin vooral kwalitatief: patiënten presteren op kwantitatieve psychometrische tests vaak nog binnen de normale grenzen, maar hebben meer tijd nodig en zijn sneller moe. Niet-cognitieve verschijnselen staan in de vroegste stadia van de ziekte van Alzheimer vaak op de voorgrond: de patiënten zijn psychisch kwetsbaar, angstig, passief, afhankelijk van de omgeving, teruggetrokken en minder spontaan. Deze niet-cognitieve aspecten zijn echter veel minder onderzocht dan de cognitieve verschijnselen. De eerste neuropsychologische stoornissen betreffen de uitgestelde herinnering, de vloeiendheid van de taal (fluency) en de snelheid waarmee informatie wordt verwerkt. Een conclusie uit het literatuuroverzicht is dat de diagnose 'beginnende ziekte van Alzheimer' vooral gebaseerd is op een kwalitatief klinisch oordeel en op verschillende metingen van het cognitief functioneren in de tijd. Het is evenwel nog onduidelijk welke symptomen zich het beste lenen om in de tijd vervolgd te worden, en hoe lang de periodes tussen de tests zouden moeten zijn. In de laatste decennia zijn er verschillende voorstellen gedaan om tot een classificatie te komen van patiënten met lichte cognitieve stoornissen. Deze worden eveneens in dit hoofdstuk besproken, waarbij met name gelet wordt op de betrouwbaarheid en de voorspellende waarde (predictieve validiteit) van de verschillende concepten. Belangrijke problemen bij het diagnostiseren van lichte dementie zijn verder het onderscheid met normale veroudering, veroudering-gerelateerde geheugenstoornissen en depressie, de variatie in de verschijningsvormen en de invloed van bijkomende ziektes en de leeftijd op de diagnostiek.

De onderwerpen van de onderzoeken uit dit proefschrift betreffen drie hoofdthema's, namelijk de invloed van de discipline die de diagnose stelt, klinische kenmerken van patiënten met cognitieve stoornissen bij wie geen sprake is van dementie, en klinische kenmerken van patiënten met dementie.

\section{De invloed van de discipline die de diagnose dementie stelt}

Gezien het feit dat de diagnose dementie in belangrijke mate is gebaseerd op het klinisch oordeel, is het opmerkelijk dat nauwelijks onderzocht is in hoeverre de discipline van de diagnosticus hierop van invloed is. Een experimenteel onderzoek naar de verschillen tussen de disciplines in het diagnostiseren van cognitieve syndromen wordt beschreven in boofdstuk 3 . Hiertoe werd er onder de deelnemers aan de Nederlandse consensus-vergadering over dementie een enquête gehouden. Vijfentachtig clinici, afkomstig uit verschillende disciplines, diagnostiseerden tien 'papieren' casus van tien verschillende patiënten met cognitieve stoornissen. Ten aanzien van de syndromale diagnose (dementie of geen dementie) werden er geen verschillen tussen de disciplines gevonden. Ten aanzien van de etiologische diagnose bleken er echter wèl significante verschillen te bestaan: neurologen bleken daarbij de 'gouden standaard' diagnosen meer te benaderen dan psychiaters. In geval van lichte dementie liepen de meningen sterker uiteen dan in ernstiger gevallen. Men was het vaker onderling oneens wanneer er ook sprake was van depressieve symptomen. De diagnostische uitspraak bleek mede bepaald te zijn door de discipline die men vertegenwoordigde. Ten 
slotte bleek dat het aantal verschillende termen die door de respondenten werden gebezigd in omgekeerde zin samenhing met de ernst van de cognitieve stoornissen.

In boofdstuk 4 wordt een onderzoek beschreven bij 430 patiënten naar de verschillen tussen de diagnosen van de (multidisciplinair werkende) Maastrichtse polikliniek voor geheugenstoornissen en die van (in het algemeen monodiscipliniar werkende) verwijzers, vooral neurologen, psychiaters en huisartsen. Zestig van de 152 patiënten met dementie waren door de verwijzer niet eerder als zodanig gediagnostiseerd (sensitiviteit: 0.61). Dit betrof vooral patiënten met lichte dementie en patiënten die tevens depressieve verschijnselen hadden. Van de 278 patiënten die niet voldeden aan de criteria voor dementie, bleken er 19 eerder wel als dement te zijn gediagnostiseerd (specificiteit: 0.93). Bij de patiënten die door psychiaters werden verwezen werden vaak de diagnosen 'dementie' en 'ziekte van Alzheimer' gemist; bij de patiënten die door neurologen waren verwezen werd de diagnose 'depressie' vaak niet gesteld.

In het hierboven beschreven onderzoek golden de diagnosen van de geheugenpolikliniek als de gouden standaard waarmee de verwizers werden vergeleken. Om na te gaan of een dergelijke handelswijze geoorloofd was, werd er een onderzoek uitgevoerd naar de voorspellende waarde van de diagnose dementie, zoals die was gesteld door de polikliniek voor geheugenstoornissen (boofdstuk 5). Hiertoe werden diverse beschikbare gegevens verzameld over het beloop van het cognitief en algemeen functioneren van 114 demente patiënten, 81 met de ziekte van Alzheimer en 33 met vasculaire dementie. De meeste patiënten waren ten tijde van het eerste onderzoek op de polikliniek voor geheugenstoornissen slechts licht of matig dement. Na een gemiddelde periode van 41 maanden (16.7-71.2), bleken 109 van de 114 patiënten (95\%) in cognitief en/of sociaal opzicht slechter te functioneren dan bij het eerste onderzoek. De geldigheid van de diagnose dementie, zoals die door de geheugenpolikliniek werd gesteld, wordt hiermee ondersteund.

De hier beschreven onderzoeken bevestigen de bekende ideeën over de diverse disciplines: psychiaters en psychologen letten vooral op het voorkomen van depressie, neurologen kjiken vooral naar hersenpathologie en verpleeghuisartsen en geriaters letten vooral op potentieel behandelbare médische aandoeningen, zoals polypharmacie. Dit zijn leder voor zich natuurlijk belangrijke aspecten, maar hier staat tegenover dat ieder specialist ook zijn (haar) eigen blinde vlekken had: de psychiaters verrichtten onvolledig lichamelijk onderzoek, de neurologen en de andere somatisch geörienteerde clinici mistten juist vaak psychiatrische stoornissen. Kennelijk heeft ieder specialisme zijn sterke en zijn zwakke kanten in de benadering van de patiënt met cognitieve stoornissen. Uiteraard kan men niet van de verschillende disciplines verwachten dat ze expert zijn op het gebied van hun collega's. Wèl nopen de resultaten van dit onderzoek tot meer bescheidenheid, want kennelijk is er geen specialisme dat alle wijsheid in dezen in pacht heeft. Neurologen, psychiaters en neuropsychologen vullen elkaar vooral bij lichte dementie goed aan. Internisten en geriaters kunnen een waardevolle bijdrage leveren bij de oudere patiënt met meer bijkomende lichamelijke aandoeningen.

Het is niet verwachten dat de diagnose 'lichte dementie' betrouwbaar gesteld kan worden met behulp van een eenvoudige screeningstest, maar evenmin is de diagnose slechts 
weggelegd voor een handjevol superspecialisten die de beschikking hebben over zeer gespecialiseerde onderzoeksmethoden. In feite was het belangrijkste verschil tussen de diagnostische benadering van de geheugen-polikliniek en die van de verwijzers de geïntegreerde multidisciplinaire aanpak en de consequente toepassing van algemeen geaccepteerde diagnostische criteria. Voor de diagnose moeten vele gegevens van uiteenlopende aard in beschouwing worden genomen. Zo bleek bijvoorbeeld dat verwijzers te weinig letten op de functionele betekenis van de cognitieve stoornissen, zoals dat door het $\mathrm{C}$ criterium van de DSM-III-R bijvoorbeeld wordt vereist.

De bevindingen van deze onderzoeken pleiten voor een meer systematisch gebruik van een multidisciplinaire benadering bij patiënten met cognitieve stoornissen. Een dergelijke multidisciplinaire aanpak hoort evenwel meer te zijn dan alleen maar de som van de afzonderlijke delen. Er dient meer nadruk gelegd te worden op de integratie van de verschillende disciplines onderling: specialisten zouden meer van elkaars werkwijzen, referentiekaders en terminologie op de hoogte moeten zijn. Studenten en assistenten zouden bij het onderwijs in de verschillende specialismes meer dan thans het geval is geoefend moeten worden in de integratie van de bevindingen van het eigen vak met die van hun collegae.

\section{Klinische aspecten van niet- demente patiënten met cognitieve verschijnselen.}

Het is algemeen bekend dat klachten over het geheugen en andere cognitieve functies in het kader van veel verschillende organische stoornissen voor kunnen komen. Het is echter veel minder bekend dat cognitieve stoornissen ook een veel voorkomend verschijnsel zijn bij diverse functionele psychiatrische stoornissen. In de dagelijkse praktijk wordt er bij het onderzoek naar psychiatrische oorzaken voor geheugenklachten vijjwel uitsluitend aandacht gegeven aan depressie. Het onderzoek dat in boofdstuk 6 wordt beschreven, werd uitgevoerd om na te gaan in hoeverre ook andere psychiatrische stoomissen dan depressie voorkomen bij patiënten met geheugenklachten. Vierhonderddertig patiënten van de Maastrichtse polikliniek voor geheugenstoomissen (242 mannen en 188 vrouwen, gemiddelde leeftijd 61,7 jaar) werden onderzocht volgens een gestandaardiseerd diagnostisch protocol, dat onder meer een lichamelijk, een neurologisch, een psychiatrisch en een neuropsychologisch onderzoek inhield, Psychiatrische stoornissen werden gediagnostiseerd volgens de criteria van de DSMIII(-R). Van de 152 patiënten met dementie hadden er 34 tevens een depressief syndroom en 19 een andere bijkomende psychiatrische stoornis. Van de 37 patiënten met andere psycho-organische stoornissen hadden er 16 een organische stemmingsstoornis, 21 andere psychopathologie. Bij de overige 241 patiënten werden er bij 152 in het totaal 159 functionele psychiatrische diagnosen gesteld: in 100 gevallen betrof dat een stemmingsstoornis, in 59 andere stoornissen, vooral angst-, aanpassings- en persoonlijkheidsstoornissen. In het totaal betroffen maar liefst $40 \%$ van alle psychiatrische aandoeningen een andere stoornis dan depressie of dysthymie. Hoewel stemmingsstoornissen dus de meest voorkomende psychiatrische stoornissen waren in deze populatie, waren er diverse andere 
psychiatrische aandoeningen in het kader waarvan geheugenproblemen ook konden voorkomen. Het psychiatrisch onderzoek bij patiënten met geheugenklachten dient derhalve niet uitsluitend gericht te zijn op het al dan niet vaststellen van depressieve verschijnselen. Bovendien kunnen psychopathologie, cognitieve symptomen en onderliggend substraat op verschillende wijzen met elkaar samenhangen. Aan de hand van een aantal casus wordt duidelijk gemaakt dat een te lineair causale visie hierop (depressie als oorzaak van cognitieve stoornissen) geen recht doet aan de ingewikkelde relatie die er kan bestaan tussen cognitie, emoties en substraat. Het belang van een goed uitgevoerd psychiatrisch onderzoek bij de patiënt met geheugenstoornissen wordt hiermee onderstreept.

Ook uit wetenschappelijk oogpunt is het van belang om onderzoek te doen bij patiënten met de combinatie van psychiatrische verschijnselen en cognitieve stoornissen, omdat er aanwijzingen zijn dat het bij hen relatief vaak zou kunnen gaan om de allereerste manifestaties van de ziekte van Alzheimer. Uit longitudinaal onderzoek is gebleken dat de emotionele veranderingen die in het kader van beginnende ziekte van Alzheimer worden gezien vaak niet zo ernstig zijn als bij een depressie in engere zin. Deze toestand voldoet betrekkelijk vaak aan de criteria van dysthymie volgens de DSM-III-R. In boofdstuk 7 wordt een onderzoek beschreven naar het cognitief functioneren van 25 dysthyme patiënten van middelbare leeftijd die in verband met geheugenklachten werden verwezen naar de polikliniek voor geheugenstoornissen. Ten aanzien van het primaire geheugen werden geen duidelijke verschillen tussen de patiënten en de contrôle-groep gevonden. Wat het secondaire geheugen betreft presteerden deze patiënten echter gemiddeld slechter dan 25 normale proefpersonen met dezelfde leeftijd, geslachtsverdeling en opleidingsniveau. Verder bleek dat de dysthyme patiënten sprake was van een vertraagd geheugen-zoekproces en van een verhoogde afleidbaarheid voor taak-irrelevante stimuli. Deze bevindingen suggereren dat althans deze groep van dysthyme patiënten wordt gekenmerkt door problemen bij inspanning vereisende cognitieve processen. Dit gaat dan ten koste van tijd en (mentale) energie, en leidt dan tot de subjectieve klachten van dysthymie.

Recente neuropsychologische concepten hebben het mogelijk gemaakt om geheugenprocessen te ontrafelen in verschillende psychologische operaties, die ieder afzonderlijk kunnen worden onderzocht, zoals aandacht, snelheid van informatieverwerking, gevoeligheid voor storende stimuli in de omgeving, het gebruik van strategieën bij het inprenten en opdiepen van informatie, en het automatisch versus gecontroleerd verwerken van informatie. Hiermee wordt een beter inzicht verkregen in de dynamiek van de psychische veranderingen bij het begin van de ziekte van Alzheimer dan met de tests die traditioneel worden gebruikt in de dagelijkse praktijk, waar nog altijd vrijwel uitsluitend begrippen als korte- en langetermijn geheugen worden gehanteerd. De bevindingen van het onderzoek in hoofdstuk 7 illustreren dit: alle onderzochte patiënten scoorden binnen de normale grenzen op de MiniMental State Examination, een veel gebruikte maar statische en weinig belastende test voor mensen die niet dement zijn. Bij de geheugenvergelijkingstaak bleek dat, naarmate het geheugen meer werd belast, de prestaties van de dysthyme patiënten steeds meer achterbleven bij die van de normale proefpersonen. Het profiel van de cognitieve stoornissen bij de patiënten uit dit onderzoek vertoont overeenkomsten met dat wat op oudere leeftijd 
verwacht kan worden. Het is derhalve mogelijk dat het hier gaat om een versterkt leeftijdseffect wat het cognitief functioneren betreft. Dit onderzoek kan geen uitsluitsel geven over de vraag in hoeverre de patiënten inderdaad lijden aan een beginnende primair neurodegeneratieve aandoening zoals de ziekte van Alzheimer. Hiervoor moeten de resultaten afgewacht worden van een longitudinaal onderzoek, dat thans in de polikliniek voor geheugenstoornissen wordt uitgevoerd. Of de bevindingen gegeneraliseerd kunnen worden naar andere dysthyme patiënten kan met dit onderzoek evenmin worden beantwoord omdat er waarschijnlijk sprake is van een selectie van patiënten: allen werden immers naar een geheugenpolikliniek verwezen in verband met cognitieve klachten.

Om meer te weten te komen over de voorstadia van dementie en over de samenhang tussen cognitieve en psychische veranderingen werd een follow-up-onderzoek uitgevoerd bij 65 patiënten mèt geheugenklachten, maar zònder dementie. Dit onderzoek wordt in boofdstuk 8 beschreven. Na een periode van gemiddeld $25.0 \pm 4$ maanden konden 47 patiënten weer opnieuw worden onderzocht. Patiënten bij wie follow-up onderzoek plaats kon vinden hadden lage uitgangs-scores op de depressie-schaal van Hamilton dan de patiënten die niet vervolgd konden worden. Er was dus sprake van enige selectieve uitval uit het onderzoek. Zes van de 47 patiënten die werden vervolgd werden als dement gediagnostiseerd bij follow-up. Dit aantal was een veelvoud van hetgeen mocht worden verwacht op grond van bekende incidentiegegevens. Patiënten die dement waren geworden waren gemiddeld ouder en hadden slechtere prestaties op de geheugenmaten dan degenen die niet dement waren geworden, maar er werd verder geen verschil gevonden in score op de depressieschaal of op de maten voor de cognitieve en de sensomotorische snelheid. Het bleek dat de mate van stemmingsstoornissen niet altijd parallel liep met de mate waarin cognitieve stoornissen aanwezig waren: sommige patiënten bleken na twee jaar te zijn verbeterd, zowel in cognitief opzicht als wat hun stemming betreft; anderen waren juist in cognitief opzicht verslechterd, met gelijke scores op de depressieschaal. Deze resultaten suggereren dat stemmingsstoornissen bij sommige patiënten de oorzakk zijn voor de cognitieve stoornissen, maar bij anderen juist het gevolg.

Een aantal methodologische problemen doen zich voor bij dit onderzoek. Zoals gezegd wis de uitval het onderzoek selectief. Dit probleem lijkt inherent te zijn aan het type onderzoek, waarbij juist de personen bij wie de sterkste verdenking op beginnende dementie bestaat, het meest onzeker zijn bij het onderzoek, en dus eerder van verdere deelname zullen afzien. Bovendien ontbrak een contrôlegroep en konden bij een aantal patiënten niet alle gegevens worden verkregen, omdat men bijvoorbeeld de taken te moeilijk vond en deze voortijdig moest beeindigen. Hierdoor is het niet goed mogelijk 'harde' conclusies te trekken uit de bevindingen van dit onderzoek. Een grotere groep van patiënten wordt thans een langere periode vervolgd. Wel kan op basis van onze ervaringen met dit onderzoek worden gesteld dat de geheugenpolikliniek een geschikte voorziening is om dit soort onderzoek uit te voeren.

Afgezien van de mogelijke relatie tussen de cognitieve stoornissen en eventuele dementie, is er nog een andere reden waarom het cognitief functioneren bij psychiarrische stoornissen meer aandacht verdient dan tot dusver het geval is. Cognitieve stoornissen bij functionele 
aandoeningen worden te vaak nog beschouwd als een soort epifenomeen, hooguit relevant omdat ze nu eenmaal in de DSM-III criteria staan. Het is van belang erop te wijzen dat alle patiënten die in onze polikliniek voor geheugenstoornissen werden onderzocht in eerste instantie hulp zochten voor hun cognitieve problemen en niet voor hun psychiatrische toestand. Cognitieve stoornissen kunnen het functioneren van de patiënt aanzienlijk beinvloeden. Bovendien zien veel patiënten hun geheugenklachten als een eerste teken van dementie, hetgeen een extra bron van ongerustheid vormt. Het ontwikkelen van therapeutische interventies die zich richten op het omgaan met deze cognitieve stoornissen is een tot dusver verwaarloosd gebied. Geheugenpoliklinieken kunnen ook in dit opzicht van waarde zijn.

\section{Klinische aspecten van patiënten met dementie}

Het onderscheid tussen de ziekte van Alzheimer en vasculaire dementie werd tot voor kort vooral gemaakt op basis van de kliniek. Vasculaire dementie werd vooral gediagnostiseerd met behulp van één van de diverse ischemische scores, waarin een aantal klinische criteria verzameld zijn. Niet lang geleden zijn er echter andere, meer omvattende, criteria voor vasculaire dementie voorgesteld. Het verschil tussen deze nieuwe en de oude criteria is, dat in de nieuwe voorstellen rekening wordt gehouden met gegevens van neuroradiologische technieken zoals CT en MRI, en dat aandacht wordt gegeven aan de relatie in de tijd tussen de dementie en eventuele beroertes. Het introduceren van nieuwe criteria voor vasculaire dementie heeft rechtstreeks gevolgen voor de diagnose 'ziekte van Alzheimer', omdat deze diagnose vooral gesteld wordt door andere aandoeningen uit te sluiten. Vasculaire dementie is de meest voorkomende vorm van dementie die moet worden uitgesloten: de bekende NINCDS/ADRDA criteria voor de ziekte van Alzheimer adviseren de ischemische score van Hachinski hiervoor te gebruiken.

Hoofdstuk 9 beschrift een onderzoek waarin zes verschillende maar veel gebruikte criteria voor vasculaire dementie werden toegepast bij 109 patiënten met dementie. Deze criteria waren: de ischemische scores van respectievelijk Hachinski, Rosen en Loeb, de criteria van de DSM-II-R, de criteria van Erkinjuntil et al., en die van de State of California Alzheimer's Disease Diagnostic and Treatment Centres (ADDTC). Afhankelijk van welke criteria werden toegepast, varieerde de mate waarin vasculaire dementie in deze groep voorkwam van 13 tot $33 \%$, en de ziekte van Alzheimer van 54 tot $64 \%$. De ischemische scores van Hachinski en Rosen leidden tot het hoogste aantal diagnosen vasculaire dementie. Het aantal patienten met vasculaire dementie nam duidelijk af wanneer de neuroradiologische gegevens en de tijdsrelatie bij de diagnostiek werden betrokken. Deze aspecten zijn belangrijk en op grond hiervan lijken de criteria van Erkinjuntii en die van de ADDTC de voorkeur te genieten. Dit onderzoek laat evenwel geen uitspraken toe over de validiteit van de verschillende criteria. Zodra de validiteit van de nieuwe criteria bevestigd is, dienen de NINCDS/ADRDA-criteria voor de diagnose ziekte van Alzheimer op dit punt te worden aangepast. 
Het onderzoek beschreven in boofdstuk 10 richtte zich op de wijdverbreide opvatting dat depressie, aanwezig ziektebesef en een behouden persoonlijkheid differentiaal-diagnostisch meer pleiten voor vasculaire dementie dan voor de ziekte van Alzheimer. De meeste onderzoeken hiernaar werden uitgevoerd bij opgenomen patiënten met ernstige dementie, en in het algemeen werd daarbij onvoldoende rekening gehouden met factoren als leeftijd, geslacht en ernst van de dementie. Ten aanzien van deze aspecten werden 48 patiënten met vasculaire dementie en 48 patiënten met de ziekte van Alzheimer, met dezelfde gemiddelde leeftijd, geslachtsverdeling en ernst van de dementie, onderling vergeleken. De meeste patiënten waren licht dement. De twee groepen verschilden onderling niet in de mate waarin depressie voorkwam en hadden vergelijkbare scores op schalen waarmee de mate van depressieve symptomen, de mate van ziektebesef en de mate van persoonlijkheids. veranderingen werden gemeten. Op grond hiervan concludeerden wij dat deze aspecten geen bijzondere waarde hebben bij de differentiële diagnostiek tussen vasculaire dementie en de ziekte van Alzheimer.

Hoofdstuk 11 beschrijft een onderzoek naar de relatie tussen ziektebesef en depressie bij demente patiënten. Het wordt vaak aangenomen dat demente patiënten depressief worden wanneer zij besef hebben van hun aftakelingsproces, maar empirisch onderzoek hierover is er nauwelijks. Om na te gaan of depressieve verschijnselen inderdaad meer voorkomen bij patiënten die blijk geven van enig besef van hun deterioratie, onderzochten wij 170 demente patiënten, 103 met de ziekte van Alzheimer, 43 met vasculaire dementie en 24 met verschillende andere oorzaken. De mate van ziektebesef werd vastgelegd op een vierpuntsschaal, waarmee discrepanties tussen de anamnese van de patiënt en die van de begeleider (gebruikt als referentie) werden nagegaan. Het bleek dat de mate waarin ziektebesef aanwezig was omgekeerd samenhing met de ernst van de dementie, maar niet met de het voorkomen van depressie of de score op de depressie schaal van Hamilton. Het item 'psychische angst' van deze Hamilton-schaal hing daarentegen wèl significant samen met het ziektebesef. Angstverschijnselen en andere niet-affectieve psychische symptomen zijn veel minder dan depressie onderzocht bij demente patiënten. Het is van belang uitgebreider onderzoek te doen naar het voorkomen van angstsymptomen bij dementie, omdat het verband met een intact ziektebesef suggereert dat deze symptomen vooral psychologisch bepaald zouden kunnen zijn. Depressie bij dementie zou daarentegen meer biologisch bepaald kunnen zijn. Als dit waar is, heeft dit gevolgen voor de wijze waarop deze verschijnselen therapeutisch moeten worden benaderd.

De onderzoeken beschreven in de hoofdstukken 10 en 11 laten nog een belangrijk methodologisch punt zien, namelijk het belang rekening te houden met leeftijd en ernst van dementie bij het vergelijken van klinische kenmerken van verschillende types dementie. Aanvankelijk verschilden de patiënten met de ziekte van Alzheimer uit ons onderzoek wèl van die met vasculaire dementie, maar dit verschil verdween nadat voor bovengenoemde variabelen was gecontroleerd. In de betreffende literatuur is tot dusver onvoldoende rekening gehouden met dit gegeven.

Hoewel cognitieve aspecten bij dementie veel meer aandacht genieten dan de nietcognitieve, zijn het vooral deze laatste symptomen die voor de verzorgers sterk belastend 


\section{SAMENVATTING}

zijn. Dat er niettemin nauwelijks onderzoek gedaan is naar deze niet-cognitieve symptomen, heeft wellicht te maken met het feit dat deze veel moeilijker te meten zijn. Daarom is het noodzakelijk dat er verfijndere instrumenten worden ontwikkeld waarmee de niet-cognitieve symptomen zowel kwantitatief als kwalitatief nauwkeuriger vast te stellen zijn. Zolang deze instrumenten er nog niet zijn, moeten wij varen op de nuances van het klinisch onderzoek. 


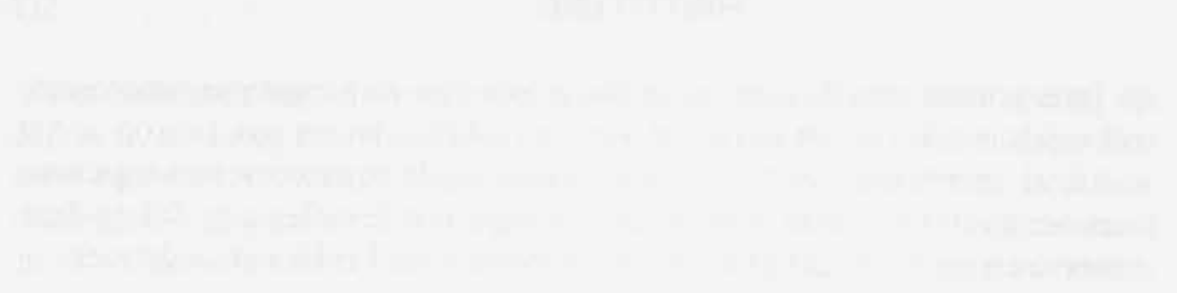




\section{References}

1. Aevarsson 0 and Skoog I. Prodromal symptoms of dementia. Neurobiol Aging 1992; 13: S5.

2. Akisal HS. Dysthymic disorder: psychopathology of proposed chronic depressive subtypes. Am J Psychiat 1983; 140: 11.20.

3. Albert ML, Feldman RG and Willis AL, The "subcortical dementia" of progressive supranuclear palsy. J Neurol Neurosurg Psychiat 1974; 37(121-130).

4. Almeida OP, Hill K, Howard R, O'Brien J and Levy R. Demographic and clinical features of patients attending a memory clinic. Int J Geriat Psychiat 1993; 8: 497-502.

5. Alzheimer A. Ueber eine eigenartige Erkrankung der Hirnrinde. Allgemeine Zeitschr fuer Psychiatrie 1907; 64: 146-148.

6. American Psychiatric Association. Diagnostic and Statistical Manual of Mental Disorders. 3rd ed. 1980; Washington: American Psychiatric Association.

7. American Psychiatric Association. Diagnostic and Statistical Manual of Mental Disorders. 3rd, revised ed. 1987; Washington: American Psychiatric Association.

8. American Psychiatric Association task force on Alzheimer's disease. The Alzheimer's disease imperative: the challenge for psychiatry. Am J Psychiat 1988; 145: 1550-1551.

9. Anonymous. Denth of a mind: a study into desintegration. Lancet 1950; i: 1012.

10. Anonymous. Memory testing: no thermometers available. Lancet 1987; i: 604-606.

11. Barker $A$ and Jones R. Age-Associated Memory Impairment: diagnostic and treatment issues. Int J Geriat Psychiat 1993; 8: 305-311.

12. Barr $A$, Benedict $R$, Tune $L$ and Brandt J. Neuropsychological differentiation of Alzheimer's disease from Vascular dementia. Int J Geriat Psychiat 1992; 7:621-627.

13. Barry PP and Moskowitz MA. The diagnosis of reversible dementia in the elderly: a critical review. Arch Int Med 1988; 148: 1914-1918.

14. Bayer $\mathrm{A}]$, Pathy J and Twining $\mathrm{C}$. The memory clinic, a new approach for the detection of early dementia. Drugs 1987; 33, suppl 2: 84-89. 
15. Benson BF. Subcortical dementia: a clinical approach. In: The dementias, R. Mayeux and R.W. G, Editor. 1983; Raven Press: New York.

16. Berg L, Danziger WL, Storandt M, Coben L, Gado M, Hughes CP, Knesevich JW and Botwinick J. Predictive features in mild senile dementia of the Alzheimer type. Neurology (Cleveland) 1984; 34: 563-569.

17. Berg L, Miller JP, Storandt MA, Duchek J, Morris JC, Rubin EH, Burke WJ and Coben LA. Mild senile dementia of the Alzheimer type: 2. Longitudinal assessment. Ann Neurol 1988; 23:477484.

18. Berg L, Smith DS, Morris JC, Miller JP, Rubin EH, Storandt M and Coben LA. Mild dementia of the Alzheimer type: 3. Longitudinal and cross sectional assessment. Ann Neurol 1990; 28:648-652.

19. Bergmann K, Kay D, Foster EM, McKechnie AA and Roth M. A follow up study of randomly selected community residents to assess the effects of chronic brain syndrome and cerebrovascular disease. In: New prospects in the study of mental disorders in old age. Proceedings of the 5 th world congress of psychiatry, E. Medica, Editor. 1971; Amsterdam. 856-865.

20. Beringer K and Mallison R. Vorseitige Versagenzustände. Allgemeines Zeitschrift fuer Psychiatrie 1949; 124: 100-130.

21. Berrios GE. Dementia during the seventeenth and eighteenth centuries: a conceptual history. Psychol Med $1987 ; 17: 829-837$.

22. Berrios GE. Non-cognitive symptoms and the diagnosis of dementia: history and dinical aspects. Brit J Psychiat 1989; 154 (suppl 4): 11-16.

23. Blackford RC and LaRue A. Criteria for diagnosing age-associated memory impairment: proposed improvements from the field. Dev Neuropsychol 1989; 5(4): 295-306.

24. Blessed $G$, Tomlinson $B$ and Roth $M$. The association between quantitive measurements of dementia and the senile changes in the cerebml grey matter of elderly subjects. Br J Psychiat 1968; 114: 797-811.

25. Bolla $\mathrm{KI}$, Lindgren $\mathrm{KN}$, Bonnacorsy $\mathrm{C}$ and Bleecker ML. Memory complaints in older adults. Fact or fiction? Arch Neurol 1991; 48: 61-64.

26. Boller F, Lopez OL and Moossy J. Diagnosis of dementia: dinicopathologic correlations. Neurology 1989; 39; 76-79.

27. Bondareff W, Mountjoy CQ, Roth $M$, Rossor MN, Iversen LL and Reynolds GP. Age and histopathologic heterogeneneity in Alzheimer's disease. Arch Gen Psychiat 1987; 44: 412-417.

28. Botwinick J. Neuropsychology of Aging. In: Handbook of Clinical Neuropsychology, S.B. Filskov and T.J. Boll, Editor. 1981; John Wiley: New York. 135-171.

29. Bozolla FG, Gorelick PB and Freels S. Personality changes in Alzheimer's Disease. Arch Neurol 1992; 49: 297 . 300.

30. Brand $\mathrm{N}$ and Jolles J. Leaming and retrieval rate or words presented auditorly and visually. J Gen Psychol 1985; 112: 201-210.

31. Brand $\mathrm{N}$ and Jolles J. Information processing in depression and anxiety. Psychol Med 1987; 17: 145-153.

32. Brayne $C$ and Calloway $P$. Normal ageing, impaired cognition, and senile dementia of the Alzheimer type: a continuum? Lancet 1988; i: 1265-1267.

33. Brust JCM. Vascular dementia is overdiagnosed. Arch Neurol 1988; 45: 799-801.

34. Burke WJ, Miller JP, Rubin EH, Morris JC, Coben LA, DuchekJ, Wittels IG and Berg L. The reliability of the Washington University Clinical Dementia Rating. Arch Neurol 1988; 45:31-32.

35. Bums A, Jacoby R and Ley R. Psychiatric phenomena in Alzheimer's disease. III: Disorders of mood. Brit J Psychiat 1990; 157: 81-86.

36. Bums A, Jacoby R and Lew R. Progression of cognitive impairment in Alzheimer's disease. J Am Geriat Soc 1992; 39: 39-45. 
37. Caine ED. Should aging-associated cognitive decline be included in DSM-IV? J Neuropsychiat Clin Neurosc 1993; 5: 1-5.

38. Cammen TJMvd, Simpson JM, Fraser RM, Preker AS and Exton-Smith AN. The memory clinic. A new approach to the detection of dementia. Brit J Psychiat 1987; 150: 359-364.

39. Cammen TJMvd, Wright G, Fraser RM, Simpson JM, Rai GS and Exton-Smith AN. Review of the work of the memory clinic after two years. In: Third Congress of the International Psychogeriatric Association. 1987; Chicago, llinois:

40. CBO. Dutch Consensus Development Conference: Diagnosis of the Dementia syndrome. 1988; Utrecht: $\mathrm{CBO}$.

41. Chatteriee A, Strauss ME, Smyth KA and Whitehouse PJ. Personality changes in Alzheimer's disease. Arch Neurol 1992; 49: 486-491.

42. Christensen AL. Luria's Neuropsychological Investigation. 1975; Copenhagen: Munksgaard.

43. Christensen $\mathrm{H}$. The validity of memory complaints by elderly people. Int J Geriat Psychiat 1991; 6: 307-312.

44. Chui HC. Dementia. A review emphasising dinicopathologic correlation and brain-behavior relationships. Arch Neurol 1989; 46: 806-814.

45. Chui HC, Teng EL, Henderson VW and Moy AC. Clinical subtypes of dementia of the Alzheimer type. Neurology 1985; 35: 1544-1550.

46. Chui HC, Victoroff JI, Margolin DM, Jagust W, Shankle R and Katzman R. Criteria for the diagnosis of ischemic vascular dementia proposed by the State of Califomia Alzheimer's Disease Diagnostic and Treatment Centers. Neurology 1992; 42(March 1992): 473-480

47. Clarfield AM. The reversible dementia's: do they reverse? Annals of Internal Medicine 1988; 109: 476-486.

48. Cohen RM, Weingarner $\mathrm{H}, \mathrm{Smallberg}$ SA, Pickar D and Murphy DL. Effort and cognition in depression. Arch Gen Psychiat 1982; 39: 593-597.

49. Colsher PL and Wallace RB. Epidemiologic considerations in studies of cognitive function in the elderly: methodology and nondementing acquired dysfunction. Epidemiologic Reviews 1991; 13: 1-27.

50. Commissaris CJAM, Jolles J, Reyersen van Buuren EJ and Verhey FRJ. Effecten van geheugenvoorlichting door middel van functiegerichte begeleiding (FGB). Gedrag en Gezondheid 1993; .

51. Commissaris CJAM, Verhey FRJ, Ponds RWHM, Jolles J and Kok GJ. Publieksvoorlichting over normale vergeetachtigheid en dementie. Effectiviteit van een planmatig ontwikkelde voorlichtingsbrochure. T Geront Geriat 1993; .

53. Commissie voor de Gezondheidsraad. Advies inzake psychogeriatrische ziektebeelden, in het bijzonder dementie, depressie en delirium. Ministerie van Welzjin, Volksgezondheid en Cultuur. 1988; Den Hang: .

54. Consensus conference. Differential diagnosis of dementing diseases.J Am Med assoc 1987; 258.

55. Constantidinis] and Richard J. Atheimer's disease. In: Handbook of Clinical Neurology, Vinken en Bruyn, Editor. 1985; Elseviers Science Publishers BV: Amsterdam .

56. Cooper B and Bickel H. Population screening and the early detection of dementing disorders in old age: a review. Psychol Med 1984; 14:81-95.

57. Craik FIM and Byrd M. Aging and Cognitive Deficis: the role of attentional resources. In: Aging and cognitive processes, F.M. Craik and S. Trehub, Editor. 1982; Plenum Press: New York. 191-211.

58. Crevel Jv. Clinical approach to dementia. In: Progress in brain research (70). 1986; Elseviers Science Publishers BV: Amsterdam. 3-13.

59. Crook T, Bartus RT, Ferris SH, Whitehouse P, Cohen GD and Gershon S. Age associated memory impairment: Proposed criteria and measures of clinical change. Dev Neuropsychol 1986; 2: 261-276. 
60. Crystal H, Dickson D, Fuld P, Masur D, Scott R, Mehler M, Masdeu J, Kawas C, Aronson M and Wolfson L. Clinico-pathological studies in dementia: non-demented subjects with pathologically confirmed Alzheimer's disease. Neurology 1988; 38: 1682-1687.

61. Cummings JL. Subcortical dementia. Neuropsychology, neuropsychiarty, and pathophysiology. BritJ Psychiat 1986; 149: 682-697.

62. Cummings JL. Depression in Parkinson's disease: a review. An J Psychiat 1992; 149: 443-454.

63. Cummings JL and Benson DF. Dementia, a clinical approach. 1983; London: Butterworhs;

64. Cummings JL, Miller B and Hill MA. Neuropsychiatric aspects of multi-infarct dementia and dementia of the Alzheimer type. Arch Neurol 1987; 44: 389-93.

65. Danielczyk W. Various mental behavioral disorders in Parkinson's disease, primary degenerative senile dementia and multiple infarction dementia. J Neural Transm 1983; 56: 161-176.

66. Danish Hospital Institute. Konsensus-rapport Senil Demens: report of the Danish Medical Research Counsil in cooperation with the Danish Hospital Institute. 1989; Copenhagen:.

67. Davis PE and Mumford SJ. Cued recall and the nature of the memory disorder in dementia. Brit J Psychiat 1984; 144: 383-386.

68. Dawe B, Procter A and Philpot $M$. Concepts of memory impairment in the elderly and their relationship to dementia-a review. Int J Geriat Psychiat 1992; 7: 473-479.

69. DeCarli C, Kaye JA, Horwitz B and Rapoport SI. Critical analysis of computer assisted tranverse axial tomography to study human brain in aging and dementia of the Alzheimer type. Neurology 1990; 40:872883.

70. Dening TR and Berrios GE. The Hachinski Ischaemic Score: a reevaluation. Int J Geriat Psychiat 1992; 7:585. 589.

71. Derix MMA Neuropsychological differentiation of dementia syndromes. 1991, University of Amsterdam:

72. Derix MMA, Gans Jd, Stam J and Portegies p. Mental changes in patients with ADS. Clin Neurol Neurosurg 1990; 92(215-222).

73. Devanand DP, Miller L, Richards M, Marder K, Bell K, Mayeux R and Stern Y. The Columbia University scale for psychopathology in Azheimer's disease. Arch Neurol 1992; 49:371-376.

74. Dillmann RJM. Alzheimer's disease. The concept of disease and the construction of medical knowledge. Doctoral Thesis, Free University of Amsterdam, 1990; Amsterdam: Thesis Publishers.

75. Duijn CMv Risk factors for Alzheimer's disease. 1992, Erasmus University Rotterdam:

76. Duyckaerts $C$, Delaère $P$ and Hauw $\mathrm{JJ}$. Ratings of the lesions in senile dementia of the Aleheimer type: concordance between laboratories. A European multicenter study under the auspices of EURAGE. J. Neurol Sci 1990; 97: 295-323.

77. Eisdorfer C, Cohen D, Paveza GJ, Ashford JW, Luchins DJ, Gorelick PB, Hirshman RS, Freels SA, Lew PS, Semla TP et al. An empirical evaluation of the Global Deterioration Scale for staging Alzheimer's disease Am J Psychiat 1992; 149: 190-194.

78. Emery VO and Oxman TE. Update of the dementia spectrum of depression. Am J Psychiat 1992; 149; 305 317.

79. Erkinjuntii T, Haatia M, Palo J, Sulkava R and Paetau A Accuracy of the clinical diagnosis of viscular dementia: a prospective clinical and post-mortem neuropathological study. J Neurol Neurosurg Psychiat 1988; 51: 1037. 1044 .

80. Erkinjuntii $T$, Ketonen $L$, Sulkava $R$, Vuorialho $M$ and Palo J. CT in the differential diagnosis between Alzheimer's disease and vascular dementia. Acta Neurol Scand 1987; 75:262-270.

81. Erkinjuntii $T$, Laaksonen $R$, Sulkava $R$, Syrjäläinen $R$ and Padoj. Neuropsychological differentiation between nomal aging, Alzheimer's disease and vascular dementia. Acta Neurol Scand 1986; 74:3934403. 
82. Erkinjuntii T, Sulkava R, Kovanen J and Palo J. Suspected dementia: evaluation of 323 consecutive referrals. Acta Neurol Sand 1987; 76: 359-364.

83. Erkinjuntti T. Differential diagnosis between Alzheimer's disease and vascular dementia: evaluation of common clinical methods. Acta Neurol Scand 1987; 76: 433-442.

84. Erkinjuntti T Dementia. Clinical diagnosis and differential diagnosis with special reference to multi-infarct dementia. 1988, University of Helsinki:

85. Erkinjuntti T and Hachinski VC. Rethinking Vascular Dementia. Cerebrovasc Dis 1993; 3: 3-23.

86. Eslinger P, Damasio A, Benton A and Van Allen M. Neuropsychologic detection of abnormal mental decline in older persons. J Am Med assoc 1985; 253: 670-674.

87. Peher EP, Mahurin RK, Inbody SB, Crook TH and Pirozzolo FJ. Anosognosia in Alzheimer's disease. Neuropsychiatry, Neuropsychology and Behavioral Neurology 1991; 4: 136-146.

88. Ferris SH, Flicker C, Kluger A, de Leon MJ, Mittelman M, Sinaiko E and Reisberg B. Early clinical prediction of Alzheimer's disease in mildly impaired elderly. In: Third international conference on Alzheimer's disease and related disorders. 1992; Abano Terme, Italy: Pergamon Press; 21.

89. Ferris SH, Flicker C, Reisberg B and Crook T. Age-associated memory impairment, benign forgetfulness and dementia. Diagnosis and treatment of senile dementia, ed. M. Bergener and B. Reisberg. 1989; BerlinHeidelberg: Springer Verlag. $72-82$.

90. Fillenbaum G, Heyman A, Williams K, Prosnitz B and Burchett B. Sensitivity and specificity of standardized screens of cognitive impaiment and dementia among elderly black and white community residents. Joumal of Clinical Epidemiology 1990; 43: 651-660.

91. Fillenbaum GG, Heyman A, Wilkinson WE and Haynes CS. Comparison of two screenings tests in Alzheimer's disease. The correlation and reliability of the Mini-Mental State Examination and the modified Blessed test. Arch Neurol 1987; 44: 924-927.

92. Filley $\mathrm{CM}$, Kelly J and Heaton RK. Neuropsychological features of early- and late-onset Alzheimer's disease. Arch Neurol 1986; 43: 574-576.

93. Fischer $P$, Jellinger $K$, Gatterer $G$ and Danielcyk W. Prospective neuropathological validation of Hachinski's Ischemic Score in dementias. J Neurol Neurosurg Psychiat 1991; 54: 580-583.

94. Fischer P, Simanyi M and Danielczyk W. Depression in dementia of the Alzheimer type and in multi-infarct dementia. Am J Psychiat 1990; 147: 1484-1487.

95. Fisher CM. Neurological fragments. II remarks on anosognosia, confabulation, memory, and other topics; and an appendix on self-observation. Neurology 1989; 39: 127-132.

96. Flicker C, Ferris SH and Reisberg B. Mild impaiment in the elderly: predictors of dementia. Neurology 1991; 41: $1006-1009$.

97. Folstein M, Folstein S and McHugh P. "Mini-mental State": a practical method for grading the cognitive state of patients for the clinician. J Psychiat Res 1977; 12: 189-198.

98. Folstein M and McHugh P. Dementia syndrome of depression. In: Senile dementia and related disorders, $R$. Katzman, R.D. Terry, andK.L. Bick, Editor. 1978, Raven Press: New York

99. Forette F, Henry J, Orgogozo J and al e. Reliability of clinical criteria for the diagnosis of dementia. Arch Neurol 1989; 46: 646-648.

100. Foster JR and Candace CM. Dementia. 1990; Chap 6.

101. Foster JR, Sclan S, Welkowitz.J, Boksay I and Seeland I. Psychiatric assessment in medical long care facilities: reliability of commonly used mating scales. Int J Geriat Psychiat 1988; 3: 229-233. 
102. Fratiglioni FY, Grut M, Viitanen GM and Winblad B. Clinical staging of dementia in a population survey: comparison of DSM-III-R and the Washington University Clinica Dementia Rating scale. Acta Psychiat Scan. 1992; 86: 49-54.

103. Frederiks JAM. The neurology of aging and dementia. ed. Vinken en de Bruyn. Vol. Handbook of clinical neurology (46). 1985; Amsterdam: Elseviers Science Publishers BV. Chap 11.

104. Freemon F. Evaluation of patients with progressive intellectual deterioration. Arch Neurol 1976; 33:658-9.

105. Galasko D, Corey-Bloom J and Thal L. Monitoring progression in Alzheimer's disease.J Am GeriatSoc 1991; 39: 932-941.

106. Galasko D, Klauber MR, Hofstetter R, Salmon DP, Lasker B and Thal LJ. The mini-mental State Examination in the early diagnosis of Alzheimer's disease. Arch Neurol 1990; 47: 49.52.

107. Galasko D, Kwo-on-Yuen PF, Klauber MR and Thal L. Neurological findings in Alzheimer's disease and nomal aging. Arch Neurol 1990; 47: 625-627.

108. Garcia C, Reding M and Blass J. Overdiagnosis of dementia. J Am Geriat Soc 1981; 29: $407-10$.

109. Giacobini E. Brain acetocholine-A view from the cerebrospinal fuid. Neurobiol Aging 1986; 7:392-396.

110. Godber C. Planning services for the elderly demented patient. Age Aging 1977;6:100-103.

111. Godderis J. Gerontopsychiatrie. 1985; Leuven: Acco.

112. Gool WAv. Cerebrospinal fluid markers of Alzheimer's disease. JAm Geriat Soc 1991; 39: 1025-1039.

113. Gottlieb GL, Gur RE and Gur RC. Reliability of psychiatric scales in patients with dementia of the Alzheimer type. Am J Psychiat 1988; 145: 857-860.

114. Grober E, Buschke $\mathrm{H}, \mathrm{Crystal} \mathrm{H}$, Bang $\mathrm{S}$ and Dresner R. Screening for dementia by memory testing. Neurology 1988; 38: 900-903.

115. Haase GR. Diseases presenting as dementia. In: Dementia, C.E. Wells, Editor. 1977; FA Davis Company: Philadelphia. 27-67.

116. Hachinski V. Preventable dementia: a call for action against the vascular dementias. Lancet 1992; 340:645. 648.

117. Hachinski V, Illif L, Zilkha E, Du Boulay G, McAllister V, Marshall J, Ross Russell RW and Symon L. Cerebral blood flow in dementia. Arch Neurol 1975; 32: 632-637.

118. Hachinski VC. Differential diagnosis of Alzheimer's disease: multi-infarct dementia. In: Alzheimer's disease: the standard reference, B. Reisberg, Editor. 1983; Free Press: New York. 632-637.

119. Hachinski VC, Potter $P$ and Merskey H. Multi-infarct dementia: a cause of mental deterioration in the elderly. Lancet 1974; ii: 207-210.

120. Hamilton M. A rating scale for depression. J Neurol Neurosurg Psychiat 1960; 23:56-62.

121. Han L and Birkett DP. Alzheimer's disease in the old and the young. IntJ Geriat Pyychiat 1993; 8:481.486.

122. Haxby JV, Grady CL and Koss E. Longitudinal study of cerebral metabolic asymmetries and associated neuropsychological patterns in early dementia of the Alecheimer type. Arch Neurol 1990; 47:753-760.

123. Henderson AS. The epidemiology of Alzheimer's disense. British Medical Bulletin 1986; 42:3-10.

124. Henderson AS and Huppert FA. The problem of mild dementia. Psychol Med 1984; 14:5-11.

125. Heyman A, Wilkinson WE, Hurwizz BJ and al e. Early onset Alzheimer's disease. Neurology 1987; 37: 980-984.

126. Hof PR, Bierer LM, Perl DP, Delacourte A, Búee L, Bouras C and Morrison JH. Evidence for early vulnerability of the medial and inferior aspects of the temproal lobe in a 82 year-old parient with preclinical signs of dementia. Arch Neurol 1992; 49: 946-953.

127. Hoff $\mathrm{P}$ and Hippius H. Alois Alzheimer 1864-1915. Ein Ueberblick ueber Leben und Werk anlaessich seines

125. Geburtstages. nervenarzt $1989 ; 60(332-337)$.

128. Hoffman R. Diagnostic errors in the evaluation of behavioral disorders. J Am Med assoc 1982; $248: 964667$. 
129. Holden UP and Woods RT. Reality orientation: psychological approaches to the 'confused' elderly. 2 ed. ed. C. Livingstone. 1988; Edinburgh: .

130. Hooijer $C$, Dinkgreve $M$, Jonker $C$ and Lindeboom J. Short screening test for dementia in the elderly population. I. A comparison between AMTS, MMSE, MSQ and SPMSQ. Int J Geriat Psychiat 1992; 7:559.571.

131. Horsman LT and Tilburg Wv. Pseudodementie, een achterhaald begrip. Tijdschr Psychiat 1984; 26: 458-471.

132. Houx PI Cognitive aging and health-related factors. 1991, Rijksuniversiteit Limburg:

133. Houx PJ, Vreeling FW and Jolles J. Age associated cognitive decline is related to biological life events. In: Alzheimer's disease: basic mechanisms, diagnosis and therapeutic strategies, K. lqbal, et al., Editor. 1991; John Wiley \& Sons Lid: Chichester, UK. 353-358.

134. Houx PJ, Vreeling FW and Jolles J. Rigorous health screening reduces age effect on memory scanning task. Brain Cognition 1991; 15: 246-260.

135. Howland RH. Pharmacotherapy of dysthymia: a review. J Clin Psychopharm 1991; 11: 83-92.

136. Howland RH and Thase ME. Biological studies of dysthymia. Biol Psychiat 1991; 30: 283-304.

137. Huber SJ and Paulson GW. The concept of subcortical dementia. Am J Psychiat 1985; 142: 1312-1317.

138. Huff FJ, Becker JT, Belle SH, Nebes RD, Holland AL and Boller F. Cognitive deficits and clinical diagnosis of Alzheimer's disease. Neurology 1987; 37: 1119-1124.

139. Hughes CP, Berg L, Danziger WL, Coben LA and Martin RL. A new rating scale for the staging of dementia. Brit J Psychiat 1982; 140: 566-572.

140. Huppert FA and Tym E. Clinical and neuropsychological assessment of dementia. Brit Med Bull 1986; 42: 11 18.

141. Janet P. Rapport des crises de psycholepsie avec les acces epileptiques. Les obsession et la psychasthenie. 1919 ;

142. Jellinger $\mathrm{K}$, Lassman $\mathrm{H}$, Fisher $\mathrm{P}$ and Danielczyk $\mathrm{W}$. Validation of diagnostic criteria for Alzheimer's disease. In: Alzheimer's disease: basic mechanisms, diagnosis and therapeutic strategies, K Iqbal, et al., Editor. 1991; John Wiley \& Sons Ltd: Chichester, UK.

143. Joachim Cl, Morris JH and Selkoe DJ. Clinically diagnosed Alzheimer's disease: autopy results in 150 cases. Ann Neurol 1988; 24: 50-56.

144. Jobst KA, Smith AD, Szatmari M, Molyneux A, Esiri E, Smith A, Jaskowski A, MCDonald B and Wald N. Detection in life of confimed Alzheimer's disease using a simple measurement of medial temporal lobe atrophy by computed tomography. Lancet 1992;340: 1179-1183.

145. Jolles J. Cognitive, emotional and behavioural dysfunctions in aging and dementia. In: Progress in Brain Reserrch, D.F. Swaab, et al, Editor. 1986; Elscvier Science Publishers: Amsterdam. 15-39.

146. Jolles J. The eatly diagnosis of dementia: a possible contribution from neuropsychology. Aging of the brain, ed. W.H. Gispen and J. Traber. 1986; Berlin: Springer Verlag. 84-100.

147. Jolles ]. Het neuro-weten en de geest. 1986; Maastricht: Rijksuniversiteit Limburg.

148. Jolles J, Bothmer J, Markerink M and Ravid R. Reduced phosphatidylinositol kinase activity in Azheimer's disease: effects of age and onset. Dementa 1993;4:81-86.

149. Jolles J, Houx PJ, Vreeling FW and Verhey FRJ. Cognitive aging, biological life events and primitive reflexes. Neurosci Lett 1993; : in press.

150. Jolles J and Verhey FRJ Psycho/neurogeriatrie in AZM 1986-1990. 1990, University of Limburg, Maastricht, the Netherlands:

151. Jolles J and Verhey FRJ. On the rationale behind treatment studies in cognitive aging and dementia. In: Alzheimer's disease: back to the future, E.C. Wolters and P. Scheltens, Editor. 1993; Vrije Universiteit Amsterdam: Amsterdam. 75-84. 
152. Jorm AF and Henderson AS. Possible improvements to the diagnostic criteria for dementia in DSM-III. BritJ Psychiat 1985; 145: 394-399.

153. Jorm AF, Korten $\mathrm{AE}$ and Henderson $\mathrm{AS}$. The prevalence of dementia: a quantitative integration of the literature. Acta Psychiat Scan. 1987; 76:465-479.

154. Karasu TB. Economic realities force psychiatrists to reevaluate all kinds of psychotherapies. Clinical Psychiatric News 1986; 14: 1-27.

155. Kaszniak AW, Poon LW and Riege W. Assessing Memory deficits: an information-processing approach. In: Handbook for clinical memory assessment of older adults, L.W. Poon, Editor. 1986; American Psychological Association: Washington. 108-128.

156. Katzman R. Alzheimer's disease. N Engl J Med 1986; 314:964-973.

157. Katzman R, Aronson M, Fuld P, Kawas C, Brown T, Morgenstern H, Frishman W, Gidez L, Eder H and Ooi WL. Development of dementing illnesses in an 80-year-old volunteer cohort. Ann Neurol 1989; 25: 317.324.

158. Katzman R, Lasker $B$ and Bernstein N. Advances in the diagnosis of dementia: accuracy of diagnosis and consequences of misdiagnosis of disorders causing dementia. 1988; Aging and the brain: Raven Press.

159. Katzman R and Terry RD. The neurology of aging. 1983; Philadelphia: Davis, FA

160. Kay DWK. Genetics, Alzheimer's disease and senile dementia. BritJ Psychiat 1989; 154:311-320.

161. Keen J. dementia: questions of cost and value. Int J Geriat Psychiat 1993; 8:369-378.

162. Khachaturian ZS. Diagnosis of Alzheimer's disease. Arch Neurol 1985; 42: 1097-1105.

163. Kiloh LG. Pseudodementia. Acta Psychiat Scan. 1961; 37: 336-351.

164. Klein DN, Taylor EB, Dickstein S and Harding K. The early-late onset distinction in DSM-III-R dysthymia.J Affect Dis 1988; 14: 25-33.

165. Knopman DS and Ryberg S. A verbal memory test with high predictive accuracy for dementla if the Alzheimer's type. Arch Neurol 1989; 46: 141-145.

166. Kocsis JH and Frances AJ. A critical discussion of DSM-III dysthymic disorder. Am J Psychiat 1987; 144: 1534 1542.

167. Konsensus-rapport. Senil Demens. 1989; Kobenhavn: Danish Medical Research Counsil in cooperation with the Danish Hospital Institute.

168. Koss E, Patterson MB, Ownby R, Stuckey JC and Whitehouse PJ. Memory Evaluation in Alzheimer's disease. Caregivers' appraisals and objective testing. Arch Neurol 1993; 50: 92-97.

169. Kraepelin. Psychiatrie. Ein Lehrbuch für Studierende und Ärtze. Vol. Band II. 1910; Leipzig: Barth, JA.

170. Kral VA. Neuro-psychiatric observations in an Old People's home.J Gerontol 1958; 13: 169-176.

171. Kral VA. Senescent forgetfulness: benign and malignant. J Canad Med Assoc 1962; 86: 257.260.

172. Kral VA and Emery O. Long term follow-up of depressive pseudodementia. Can J Psychiatry 1989; 34: 445 447.

173. Kral VA and Müller H. Memory disfunction, a prognostic factor in getiatric patients. Can Psychiart Assoc J 1966; 11: 343-349.

174. Kuiper PC. Hoofdsom der Psychiatrie. 1973; Utrecht: Erven J Bijleveld.

175. Kukull W, Larson E, Reifler B, Lampe T, Yerby $M$ and Hughes J. Inter-rater reliability of Azheimer's Disease diagnosis. Neurology 1990; 40: 257-260.

176. La Rue A. Memory Loss and Aging. Distinguishing Dementia from Benign Senescent Forgetfulness and Depressive Pseudodementia. Psychiatric Clinics of North America 1983; 5;89-103.

177. Landis JR and Koch GG. The measurement of observer agreement for categorical data. Biometrics 1977;3: 159-174.

178. Larson EB. Illnesses causing dementia in the very elderly. N Engl J Med 1993; 328: 203-205. 
179. Larson EB, Reifler BV, Featherstone $\mathrm{HJ}$ and English DR. Dementia in elderly outpatients: a prospective study. Ann Int Med 1984; 100: 417-423.

180. Larson EB, Reifler BV, Sumi SM, Canfield CG and Chinn NM. Diagnostic evaluation of 200 elderly outpatients with suspected dementia. J. Gerontol 1985; 40: 536-543.

181. LaRue A, Watson J and Plockin DA. First symptoms of dementia: a study of relatives' reports. Int J Geriat Psychiat 1993; 8: 239-245.

182. Lazarus LW, Newton N, Cohler B, Lesser J and Schweon C. Frequency and presentation of depressive symptoms in patients with primary degenerative dementia. Am J Psychiat 1987; 144: $41-45$.

183. Lezak MD. Neuropsychological assessment. 2nd edition ed. 1983; New York: Oxford University Press.

184. Lhermitte F. Human autonomy and the frontal lobes. Part 2: patient behaviou in complex and social situations: the environment dependency syndrome. Ann Neurol 1986; 19: 335-343.

185. Lhermitte F, Pillon B and Serdaru M. Human autonomy and the frontal lobes. Part 1: imitation and utilisation behaviour: a neuropsychological study of 75 patients. Ann Neurol 1986; 19: 326-334.

186. Lipowski ZJ. Organic mental disorders: Their history and classification with special reference to DSM-III. In: Clinical aspects of Azheimer's disease and senile dementia, N.E. Miller and G.D. Cohen, Editor. 1981; Raven Press: New York. 3745.

187. Lishman WA. Organic Psychiatry: the psychological consequences of cerebral disorder. 1987; Oxford: Blackwell Scientific. 603-605.

188. Lishman WA. Neurologists and psychiatrists. In: The bridge between neurology and psychiatry, E.H. Reynolds and M.R. Trimble, Editor. 1989; Churchill Livingstone: Edinburgh. 24-37.

189. Liston EH and La Rue A. Clinical differentiation of primary degenerative and multi-infarct dementia: a critical review of the evidence. Part I: Clinical studies. Biol Psychiat 1983; 18: 1451-1465.

190. Liston $\mathrm{EH}$ and La Rue A. Clinical differentiation of primary degenerative and multi-infarct dementia: a critical review of the evidence. Part II: Pathological studies. Biol Psychiat 1983; 18: 1466-1484.

191. Livingstone $G$, Hawkins A, Graham N, Blizard B and Mann A. The Gospel Oak study: prevalence rates of dementia, depression and activity among eldedy residents in inner London. Psychol Med 1990; 20: 137-146.

192. Loeb C. Clinical diagnosis of multi-infarct dementia. In: Aging of the brain and dementia, L. Amaducci, Editor. 1980; Raven press: New York. 251-260.

193. Loeb C and Gandolfo C. Diagnostic evaluation of degenerautve and vascular dementia. Stroke 1983; 14: 399401.

194. Lopez O, Swihart A, Becker J, Reinmuth O, Reynolds C, Fezek D and Daly F. Reliability of NINCDS-ADRDA clinical criteria for the diagnosis of Alzheimer's Disease. Neurology 1990; 40: 1517-1522.

195. Luria AR. Higher cortical functions in man. second edition ed. 1980; New York: Basic Books.

196. Mahendra B. Pseudodementia: abandon the term? Am J Psychiat 1984; 141: 471-472.

197. Marsden C and Harrison M. Outcome of investigation of patients with presenile dementia. Brit Med 1972; 2 : 249 .

198. Marsden CD. Assessment of dementia. ed. V.e. Bruyn. Vol. Handbook of clinical neurology (46). 1985; Amsterdam: Elseviers Science Publishers BV. Chap 12.

199. Mayer-Gross W, Slater E and Roth M. Clinical Psychiarry. 1969; London: Ballière Tindall \& Cassell. 593-600.

200. Mayeux $\mathrm{R}$, Stern Y and Spanton S. Heterogeneity in dementia of the Alzheimer type: evidence of subgroups. Neurology 1985; 35: 453-461.

201. McAllister TW. Cognitive Functioning in the Affective Disorders. Comp Psychiat 1981; 22: 572-586.

202. McAllister TW and Price TRP. Severe depressive pseudodementia with and without dementia. Am J Psychiat $1982 ; 139: 626.629$. 
203. McEntee WJ and Crook TH. Age associated memory impairment: a role for catecholamines. Neurology 1990; 40: $526-530$.

204. McGlone J, Gupta S, Humphrey D, Oppenheimer S, Mirsen T and Evans DR. Screening for early dementia using memory complaints from patients and relatives. Arch Neurol 1990; 47: 1189-1193.

205. McGlynn SM and Schachter DL. Unawareness of deficits in neuropsychological syndromes. J Exp Neuropsychol 1989; 1: 143-205.

206. McHugh PR and Folstein MF. Psychiatric syndromes in Huntington's chorea: a clinical and phenomenologic study. In: Psychiatric aspects of neurologic disease, D.F. Benson and D. Blumer, Editor. 1975; Grune \& Stratton: New-York. 267-286.

207. McKhann G, Drachmann D, Folstein M, Katzman R, Price D and Stadlan E. Clinical diagnosis of Alzheimer's Disease: report of the NINCDS-ADRDA workgroup under the auspices of the department of Health and Human Services task force on Alzheimer's Disease. Neurology 1984; 34: 939-944.

208. Mendez MF, Selwood AS, Mastri AR and Frey WH. Pick's disease versus Alzheimer's disease: a comparison of clinical characteristics. Neurology 1993; 43: 289-292.

209. Mesulam M. Patterns in behavioural neuroanatomy: association areas, the limbic system and hemispheric specialisation. In: Mesulam, M Principles of behaviral neurology, D. Company, Editor. 1985; Phlidelphia.

210. Miller E. Psychological approaches to the management of memory impairments. Brit J Psychiat 1992; 160: 1-6.

211. Miller W. Psychological deficits in depression. Psychol Bull 1975; 82:238-260.

212. Mohs R, Kim Y, Johns CA, Dunn D and Davis KL. Assessing changes in Alzheimer's disease: memory and language. In: Handbook for clinical memory assessment of older adults, LW. Poon, Editor. 1986; American Psychological Association: Washington. 149-155.

213. Molloy DW, Clamette RM, Mcllroy WE, Guyatt G, Rees L and Lever J. Clinical significance of primitive reflexes in Alzheimer's disease. J Am Geriat Soc 1991; 39: 1160-1163.

214. Mölsä PK, Paljärvi L, Rinne JO, Rinne UK and Säkö $\mathrm{E}$. Validity of clinical diagnosis in dementia: a prospective clinicopathological study.J Neurol Neurosurg Psychiat 1985; 48: 1085-1090.

215. Morris J, McKeel $\mathrm{D}$ and Fulling $\mathrm{K}$ Validation of clinical diagnostic criteria in senile dementia of the Alzheimer type. Ann Neurol 1988; 24: 17-22.

216. Morris JC and Fulling K Early Alzheimer's disease: diagnostic considerations. Arch Neurol 1988; 45:345-349.

217. Morris JC, Heyman A, Mohs RC, Hughes JP, van Belle G, Fillenbaum G, Mellits ED and Clark C. The Consortium to Establish a Registry for Alzheimer's Disease (CERAD). Neurology 1989; 39: 1159-1165.

218. Morris JC, Mohs R, Rogers H, Hughes JP, Belle Bv, Fillenbaum G, Mellits ED and Clakk C. CERAD dinical and neuropsychological assessment of Alzheimer's disease. Psychopharmacological Bulletin 1988; 24: 641-651.

219. Mowry BJ and Burvill PW. A Study of mild dementia in the community using a wide range of diagnostic criteria. Brit J Psychiat 1988; 153: 328-334.

220. Neary D, Snowden JS, Bowen DM, Sims NR, Mann DMA, Benton IS, Northen B, Yates PO and Davison AN. Neuropsychological syndromes in presenile dementia due to cerebral atrophy.J Neurol Neurosurg Psychiat $1986 ; 49: 163-174$.

221. Neary D, Snowden JS, Northen B and Goulding P. Dementia of frontal lobe type. J Neurol Neurosurg Psychiat $1988 ; 51: 353-361$.

222. Nelson A, Fogel BS and Faust D. Bedside cognitive screening instruments: a critical assessment.J Nerv Ment Dis $1986 ; 174$ (2): $73-83$.

223. Niederehe G. Depression and Memory Impairment in the Aged. In: Clinical Memory Assessment of older adulss, L.W. Poon, Editor. 1986; Van Nostrand Reinhold.: New York. 226-237. 
224. Nielsen H, Lolk A, Pedersen I, Autzen M, Sennef C and Kragh-Sørensen P. The accuracy of early diagnosis and predictors of death in Alzheimer's Disease and vascular dementia - a follow-up study. Acta Psychiat Scan. 1991; 84: 277-282.

225. NIH. Differential diagnosis of dementing diseases. In: NIH Consensus Development Conference. 1987; $\mathrm{NIH}$;

226. O'Brien JT, Beats B, Hill K, Howard R, Sahakian B and Levy R. Do subjective memory complaints precede dementia? A three year follow-up study of patients with supposed 'benign senescent forgetfulness'. Int J Geriat Psychiat 1992; 7:481-486.

227. O'Brien JT and Lewy R. Age associated memory impaiment. Brit Med 1992; 304: 5-6.

228. O'Brien MD. Vascular dementia is underdiagnosed. Arch Neurol 1988; 45: 797-798.

229. O'Connor DW, Pollit PA, Hyde JB, Miller ND and Fellowes JL. Clinical issues relating to the diagnosis of mild dementia in a British community survey. Arch Neurol 1991; 48: 530-534.

230. O'Connor DW, Pollit PA, Brook CPB, Reiss BB and Roth M. Does early intervention reduce the number of elderly people with dementia admitted to institutions for long term care? Brit Med 1991; 302: 871-875.

231. O'Connor DW, Pollit PA, Hyde JB, Brook CPB, Reiss BB and Roth M. Do general practitioners miss dementia in elderly patients. Brit Med 1988; 297: 1107-1110.

232. O'Connor DW, Pollit PA, Hyde JB, Fellows J, Miller ND and Roth M. A follow-up study of dementia diagnosed in the community using the Cambridge Mental Disorders of the elderly examination. Acta Psychiat Scand 1990; 81: 78-82.

233. O'Connor DW, Pollit PA, Jones BJ, Hyde JB, Fellowes JL and Miller ND. Continued clinical validation of dementia diagnosis in the community using the Cambridge Mental Disorders of the elderly examination. Acta Psychiat Scand 1991; 83: 41-45.

234. O'Connor DW, Pollitt PA, Roth M, Brook CPB and Reiss BB. Memory complaints and impairment in normal, depressed and demented elderly persons identified in a community survey. Arch Gen Psychiat 1990; 47: 224227.

235. Ott BR and Fogel BS. Measurement of depression in dementia: self vs clinician rating. Int J Geriat Psychiat 1992; 7: 899-904.

236. Parker $\mathrm{G}$, Brown L and Blignault I. Coping Behaviours as Predictors of the course of clinical Depression. Arch Gen Psychiat 1986; 43: 561-565.

237. Paulus WP, Bancher $C$ and Jellinger $K$. Interrater reliability in the neuropathologic diagnosis of Alzheimer's disease. Neurology 1992; 42: 329.332.

238. Persson G, Berg S, Nilsson L and Svanborg A Subclinical dementiá. Relation to cogntion, personality and psychopathology: a nine-year prospective study. Int j Geriat Psychiat1991; 6: 239-247.

239. Petersen RC, Smith G, Kokmen E, linik RJ and Tangalos EG. Memory function in normal aging. Neurology 1992; $42: 396.401$.

240. Petry S, Cummings JL, Hill MA and Shapira J. Personality alterations in dementia of the Alzheimer type. Arch Neurol 1988; 45: 1187-1190.

241. Philpot MP and Levy R. Memory dinic for the early diagnosis of dementia. Int J Geriat Psychiat 1987; 2: 195200.

242. Plotkin DA, Mint. JM and Jarvik LF. Subjective memory complaints in geriatric depression. Am J Psychiat 1985; 142: 1103-1105.

243. Plugge L, Verhey F, Everdingen vE and Jolles J. Differential diagnosis of dementia. An experimental study into intra- and interdisciplinary agreement. J Geriat Psychiat Neurol 1991; 4: 90-97.

244. Plugge LA, Verhey FRJ and Jolles J. Differential diagnosis of dementia: a comparison berween the expert system EVINCE and clinicians. J Neuropsychiat Clin Neurosc 1991; 3: 398-404. 
245. Ponds RWHM, Bruning HA and Jolles J. Ouderen en geheugenklachten. De rol van zelfkennis over het geheugen en depressie. Tijdschr Geront Geriat 1992; 23: 188-194.

246. Ponds RWHM, Verhey FRJ, Rozendaal N, Jolles J and Deelman BG. Dementie-screening: validiteit van de cognitive screeningstest en de mini-mental state examination. Tijdschr Geront Geriat 1992; 23: 94-99.

247. Poon LW. Differences in Human Memory and Aging: Nature, causes and dinical implications. In: Handbook of the Psychology of Aging, 2 ed., J.E. Birren and K.W. Schaie, Editor. 1985; Van Nostrand Reinhold.: New York. 427-462.

248. Popkin MK, Tucker G, Caine E, Folstein M and Grant I. The fate of organic mental disorders in DSM-VI: a progress report. Psychosomatics 1989; 30: 438-441.

249. Powers WJ, Perlmutter JS, Videen TO, Herscovitch P, Griffeth LK, Royal HD, Siegel BA, Morris JC and Berg L. Blinded clinical evaluation of positron emission tomography for diagnosis of probable Alzheimer's disease. Neurology 1992; 42: 765-770.

250. Radue EW, Boulay GHd, Harrison MJG and Thomas DJ. Comparison of angiographic and CT scan findings between patients with multiinfarct dementia and those with dementia due to primary neuronal degeneration. Neuroradiology 1978; 16: 113-115.

251. Raskin A. Partialing Out the Effects of Depression and Age on Cognitive Functions: Experimental Data and Methodological Issues. In: Clinical Memory Assessment of older adults, L.W. Poon, Editor. 1986; Van Nostrand Reinhold.: New York. 244-255.

252. Reding $M, H$, Haycox $J$ and Blass J. Depression in patients referred to a dementia clinic: a three year prospective study. Arch Neurol 1985; 42: 894-896.

253. Reifler BV, Larson $\mathrm{E}$ and Hanley R. Coexistence of cognitive impairment and depression in geriatric outpatients. Am J Psychiat 1982; 139: 623-629.

254. Reisberg B, Ferris S, Borenstein J, Sinaiko E, de Leon MJ and Buttinger C. Assessment of presenting symptoms. In: Handbook for clinical memory assessment of older adults, L.W. Poon, Editor. 1986; American Psychological Association: Washington. 108-128.

255. Reisberg B, Ferris $\mathrm{S}$, de Leon $\mathrm{M}$ and Crook $\mathrm{T}$. The global deterioration scale for assessment of primary degenerative dementia. Am J Psychiat 1982; 139: 1136-1139.

256. Reisberg B, Ferris SH, Deleon M], Sinaiko E, Franssen E, Kuger A, Mir P, Borenstein J, George AE, Shulman E et al. Stage-specific behavioral, cognitive and in vivo changes in community residing subjects with ageassociated memory impairment and primary degenerative dementia of the Alzheimer type. Drug Development Research 1988; 15: 101-114.

257. Reisberg B, Ferris SH, Kluger A, Franssen E, Deleon MJ, Mittelman M, Borenstein J, Rameshwar K and Alba R. Symptomatic changes in CNS Aging and dementia of the Alzheimer type: cross sectional, temporal and remediable concomitants. Diagnosis and Treatment of dementia, ed. Bergener and Reisberg. 1989; BerlinHeidelberg: Springer Verlag. 193-223.

258. Reisberg B, Gordon B, McCarthy M, Ferris SH and deLeon MJ. Insight and denial accompanying progressive cognitive decline in normal aging and Alzheimer's disease. In: Geniatric Psychiatry: ethical and legal issues, A.P. Press, Editor. 1985; Washington DC.

259. Reisberg B, Shulman E, Ferris $\mathrm{SH}$, de Leon $\mathrm{MJ}$ and Geibel V. Clinical assessment of age-associated cognitive decline and primary dementia: prognostic concomitants. Psychophamacology Bulletin 1983; 19:734739.

260. Reyersen van Buuren EJ and Jolles J. Function Oriented Guidance as a form of neuropsychological rehabilitation for memory disturbed patients. In: Rehabilitation of the brain injured: a neuropsychological perspective, E. Vakil, D. Hoofien, andZ.L. Groswasser, Editor. 1990; Freund Publishing: London. 87-103.

261. Risse $S$, Raskind $M$ and Nochlin D. Neuropathological findings in patients with clinical diagnosis of probable Alzheimer's disease. Am J Psychiat 1990; 147: 168-172. 
262. Roberts JKA. Differential diagnosis in neuropsychiatry. 1984; Chichester, New York Brisbane Toronto Singapore: Wiley \& Sons.

263. Roberts MA, McGeorge AP and Caird Fl. Electroencephalography and computerised tomography in vascular and non-vascular dementia. J Neurol Neurosurg Psychiat 1978; 41: 903-906.

264. Robins E and Guze SB. Establishment of diagnostic valdidty in psychiatric illness: its application to schizofrenia. Am J Psychiat 1970; 126: 983-987.

265. Robinson-Whelen $S$ and Stomndt $M$. Immediate and delayed prose recall among normal and demented adults. Arch Neurol 1992; 49:32.34.

266. Romàn GC, Tatemichi TK, Erkinjuntii T, Cummings JL, Masdeu JC, Garcia JH, Amaducci L, Orgogozo J-M, Brun A and Hofman A. Vascular dementia: diagnostic criteria for research studies. report of the NINAIREN international workshop. Neurology 1993; 43:250-260.

267. Ron MA, Toone BK, Garralda ME and Lishman WA. Diagnostic accuracy in presenile dementia. Brit J Psychiat 1979; 134: 161-168.

268. Rosen WG and Mohs RC. Evolution of cognitive decline in dementia. In: Alzheimer's disease: a report of progress, S. Corkin, Editor. 1982; Raven Press: New York. 183-188.

269. Rosen WG, Terry R, Fuld PA, Katzman R and Peck A. Pathological verification of ischemic score in the differentiation of dementias. Ann Neurol 1980; 7:486488.

270. Rosenman S. The validity of the diagnosis of mild dementia. Psychol Med 1991; 21: 923-934.

271. Roth M, Huppert FA, Tym E and Mountioy CQ. CAMDEX: the Cambridge examination for mental disorders of the lederly. 1988; Cambridge: Cambridge University Press.

272. Roth $M$, Tym E, Mountioy CQ, Huppert FA, Hendrie H, Verma S and Goddard R. CAMDEX: a standardised instrument for the diagnosis of mental disorder in the elderly with special reference to the early detection of dementia. Brit J Psychiat 1986; 149: 698-709.

273. Rowe JW and Kahn RL. Human aging: usual and succesful. Science 1987; 237: 143-149.

274. Rubin EH and Kinscherf DA. Psychopathology of very mild dementia of the Alzheimer type. Am J Psychiat 1989; 146: 1017-1021.

275. Rubin $\mathrm{EH}$, Morris JC and Berg L. The progression of personality changes in patients with mild senile dementia of the Alzheimer type.J Am Geriat Soc 1987; 35: 721.725.

276. Rubin EH, Morris JC, Grant EA and Vendegna T. Very mild senile dementia of the Alzheimer type: 1. Clinical assessment. Arch Neurol 1989; 46: 379-382.

277. Salthouse TA. Speed of behaviour and its implications for cognition. In: Handbook of the Psychology of Aging, 2 ed.J.J. Birren and K.W. Schaie, Editor. 1985; Van Nostrand Reinhold.: New York. $400-426$.

278. Schacher DL. Towards a cognitive neuropsychology of awareness: implicit knowledge and anosognosia. J Clin Experiment Neuropsychol 1990; 12: 155-178.

279. Scheltens P MR Imaging in Atheimer's disease. 1993, Free University of Amsterdam:

280. Scheitens P, Leys D and Barkhof F. Hippocampal atrophy on magnetic resonance imaging in Alzheimer's disease and normal aging. Neurology 1991; 41 (suppl 1): 341-342.

281. Schulte BPM. Consensusdiagnostiek bij het dementiesyndroom. Ned Tijdschr Geneesk 1989; 133: 981-985.

282. Selzer B and Sherwin I. A comparison of clinical features in early and late onset primary degenerative dementia: one entity or wo? Arch Neurol 1983; 40: 143-146.

283. Shiffin RM and Schneider W. Controlled and automatic human information processing. II: Perceptual leaming, automatic attending and a general theory. Psychological Review 1977; 84: 127-190.

284. Shimamura AP and Squire LR Memory and metamemory: a study of the feeling-of-knowing phenomenon in amnestic patients.J Exp Psychol 1986; 12: 452460. 
285. Siegel $\mathrm{S}$ and Castellan NJ. Nonparametric systems for the behavioral sciences. Statistical series, ed.J.D. Anker. 1988; New York: McGraw-Hill.

286. Skoog I, Nilsson L, Palmertz B, Andreasson L and Svanborg A. A population-based study of dementia in 85year olds. N Engl J Med 1993; 328: 153-158.

287. Small GW and Greenberg DA. Biological markers, genetics and Alzheimer's disease. Arch Gen Psychiat 1988; 45: 945-947.

288. Small GW and Jarvik LF. The dementia syndrome. Lancet 1982; ii: 1443-1446.

289. Small GW and Jarvik LF. DSM-III diagnosis of dementia. Am J Psychiat 1983; 140: 948.

290. Smith J and Kiloh L. The investigation of dementia: results in 200 consecutive admissions. Lancet 1981; ii: 824-827.

291. Soininen H, Puranen M and Riekkinen PJ. Computed Tomography findings in senile dementia and normal aging. J Neurol Neurosurg Psychiat 1982; 45: 50-54.

292. Spizzer R, First MB, Williams JBW, Kendler $K$, Pincus $H A$ and Tucker $G$. Now is the time to retire the term "organic mental disorders". Am J Psychiat 1992; 149: 240-244.

293. Spitzer R, Williams JBW and First M. A proposal for DSM-VV: solving the "organic-nonorganic" problem.J Neuropsychiat Clin Neurosc 1989; .

294. Stam FC. Dementie. ed. F.C. Stam. 1985; Utrecht: Bohn, Scheltema en Holkema. Chap 1.

295. Starkstein SE and Robinson RG. Affective disorders and cerebrovascular disease. Brit J Psychiat 1989; 154: $170-182$.

296. Stern Y, Mayeux R, Sano M, Hauser WA and Bush T. Predictors of disease course in patients with probable Alzheimer's disease. Neurology 1987; 37: 1649-1653.

297. Stemberg S. Memory scanning; new findings and current controversies. Q.J. Exp. Psychol. 1975; 27: 1-32

298. Stevens J. Applied multivariate statistics for the social sciences. 1986; Hillsdale, New Jersey: Lawrence Erlbaum Associates, Publishers.

299. Storandt M, Botwinick J, Danziger WL, Berg L and Hughes CP. Psychometric differentiation of mild dementia of the Alzheimer type. Arch Neurol 1984; 41: 497-499.

300. Storandt $\mathrm{M}$ and Hill RD. Very mild senile dementia of the Alzheimer type: 2. Psychometric test performance. Arch Neurol 1989; 46: 383-386.

301. Sulkava R and Amberla K. Alzheimer's disease and senile dementia of the Alzheimer type: a neuropsychological study. Acta Neurol Scand 1982; 65: 541.552.

302. Summers WK, Deboynton V, Marsh GM and Majovski LV. Comparison of seven psychometric instruments used for the evaluation of treatment effect in Alzheimer's disesse. Neuroepidemiology 1990; 9: 193-207.

303. Sunderland A, Watts K, Baddeley AD and Harris JE. Subjective memory assessment and test performance in elderly adults. J. Gerontol 1986; $41: 376-384$.

304. Sunderland T, Tarriot PM, Cohen RM, Weingartner $\mathrm{H}$, Mueller EA and Murphy DL. Anticholinergic sensitivity in patients with dementia of the Alzheimer type and age matched controls. A dose respons study. Arch Gen Psychiat 1987; 44(5): 418-426.

305. Sunderland T, Tarriot PM, Mueller EA, Murphy DL, Weingarner H and Cohen RM. Cognitive and behavioral sensitivity to scopolamine in Alzheimer patients and controls. Psychopharmacological Bulletin 1985; 21(3): 676-679.

306. Tanja TA and Hofman A. De epidemiologie van seniele dementie. Ned Tijdschr Geneesk 1985; 129: 2206 . 2209.

307. Taylor JL, Miller TP and Tinklenberg JR. Correlates of memory decline: a 4.year longitudinal study of older adults with memory complaints. Psychology and Aging 1992; 7: 185-193.

308. Teitelbaum M. Towards a better integration of medical and psychiatric care J Am Med assoc 1982; $248: 97$. 
309. Thompson MK. Myths about the care of the elderly. Lancet 1985; i: 523.

310. Tierney MC, Fisher RH, Lewis AJ, Zorzitto ML, Snow WG, Reid DW and Nieuwstraten P. The NINCDSADRDA work group criteria for the clinical diagnosis of probable dementia: a clinicopathologic study of 57 cases. Neurology 1988; 38: 359-364.

311. Todorov AB, Go RCP, Constantidinis J and Elston RC. Specificity of the clinical diagnosis of dementia.J Neurol Sciences $1975 ; 26: 81-98$.

312. Tombaugh TN and Mclntyre NJ. The Mini-Mental State Examination: a comprehensive review. J Am Geriatr Ass 1992; 40: 9222-935.

313. Tomlinson BE, Blessed $G$ and Roth M. Observations on the brains of demented old people. J. Neurol Sci 1970; 11: $205-242$.

314. Verhage F. Intelligentie en leeftijd (Intelligence and Age). 1964; Assen, the Netherlands: Doctoral dissertation.

315. Verhey FRJ and Jolles J. Over de spraakverwarring rond het begrip dementie en de ziekte van Alzheimer. Tijdschr Geront Geriat 1988; 19: 89.96.

316. Verhey FRJ, Jolles J, Ponds RWHM, Rozendaal N, Plugge L, Vet HCWd, Vreeling FW and Lugt PJMvd. Diagnosing dementia: a comparison between a monodisciplinary and multidisciplinary approach. J Neuropsychiat Clin Neurosc 1993; 5: 78-85.

317. Verhey FRJ, Plugge LA, Everdingen JEv and Jolles J. Verschillende disciplines, verschillende diagnosen? - een enquête onder de deelnemers van de consensusvergadering over dementie. Tijdschr Geront Geriat 1991; 22: 187.194.

318. Verhey FRJ, Ponds RWHM, Jolles J and Lug PJMvd. Een fobie voor vergeten: een nieuw psycho-geriatrisch syndroom. Medisch Contact 1991; 46:575-576.

319. Verhey FRJ, Reyersen van Buuren E and Jolles J. De geheugenkliniek: multidisciplinaire benadering bij stoornissen van het geheugen en andere cognitieve stoornissen. Ouder worden $\mathrm{Nu}$, ed. Houweling $\mathrm{T}$. J. 1987; Amsterdam: Gerontologisch instituut. 82-88.

320. Verhey FRJ, Reyersen van Buuren EJ and Jolles]. Neuropsychiatric disturbances in the presenium: possible contribution to early diagnosis of dementia. Clin Neurol Neurosurg 1987; 89 supl II: 22.

321. Verhey FRJ, Roozendaal N, Ponds RWHM and Jolles J. Dementia, depression and awareness. Int J Geriat Psychiat 1993; .

322. Verhey FRJ, Vreeling FW and Jolles J. DSM-III and NINCDS/ADRDA criteria for dementia and Alzheimer's disease: impact of diagnostic procedures on daly practice. In: Fifth Meeting of the Intemational Study Group on the pharmacology of Memory Disorders Associated with Aging, Zünich, january 1989. 1989; Zürich: Center for Brain Sciences and Metabolism Charitable Trust; 419-423.

323. Verwoerdt A. Individual Psychotherapy in senile dementia. In: Clinical Aspects of Alzheimer Disease and senile Dementia, N.E. Miller and G. Cohen, Editor. 1982; Raven Press: New York.

324. Vink $M$ and Jolles J. A new version of the Trail Making Test as a information processing task. J Clin Exp Neuropsychol 1985; 7: 162.

325. Vitaliano PP, RussoJ, Breen AR, Vitiello MV and Prinz PN. Functional decline in the early stages of Alzheimer's disease.J Psychol Aging 1986; 1:4146.

326. Vreeling FW, Jolles J, Verhey FRJ and Houx PJ. Primitive reflexes in Alzheimer's disease and vascular dementia. $\mathrm{J}$ Geriarric Psychiat Neurol in press; .

327. WadeJPH, Mirsen TR, Hachinski WD, Fisman M, Lau C and Merskey H. The clinical diagnosis of Alzheimer's disease. Arch Neurol 1987; 44: 24-29.

328. Walstra GJM, Derix MMA, Hijdra A and Crevel Hv. Een polikliniek voor geheugenstoornissen; eerste ervaringen. Ned Tijdschr Geneesk 1992; 136: $328-332$. 
329. Weiner MF, Bruhn M, Svelik D, Tintner R and Hom J. Experiences with depression in a dementia clinic. Journal of Clinical Psychiatry 1991; 52: 234-238.

330. Weingartner H. Automatic and Effort-demanding Cognitive Processes in Depression. In: Clinical Memory Assessment of older adults, L.W. Poon, Editor. 1986; Van Nostand Reinhold: New York. 218-225.

331. Weinstein H SPECT in Alzheimer's disease. 1992, Free University of Amsterdam:

332. Weissman MM, Leaf PJ, Bruce ML and Florio L. The epidemiology of dysthymia in five communities: rates, risks, comorbidity and treatment. Am J Psychiat 1988; 145: 815-819.

333. Wells CE. Dementia: definition and description. In: Dementia, C.E. Wells, Editor. 1977; FA. Davis Company: Philadelphia. Chap 1.

334. Welsh $\mathrm{K}$, Butters $\mathrm{N}$, Hughes J, Mohs R and Heyman A. Detection of abnormal memory decline in mild cases of Alzheimer's disease using CERAD neuropsychological measures. Arch Neurol 1991; 48: 278-281.

335. Welsh KA, Butters N, Hughes JP, Mohs R and Heyman A. Detection and staging of dementia in Azheimer's disease. Arch Neurol 1992; 49: 448-452.

336. Whalley LJ. Drug treatments of dementia. Brit J Psychiat 1989; 155:595-611.

337. Whitbourne SK. The psychological construction of life span. In: Handbook of the Psychology of Aging, J.E. Birren and K.W. Schaie, Editor. 1985; Van Nostrand Reinhold.: New York. 594618.

338. Whitehouse PJ. The concept of subcortical and cortical dementia: another look. Ann Neurol 1986; 19: 1-6.

339. Williamson J, Stokoe IH, Gray S, Fisher M, Smith A, McGhee A and Stephenson E. Old people at home: their unreported needs. Lancet 1964; 1: 1117-1120.

340. Wragg RE and Jeste DV. Overview of depression and psychosis in Alzheimer's disease. Am J Psychiat 1989; 146: $577-587$.

341. Yesavage JA. Nonpharmacologic treatments for memory losses with normal aging. AmJ Psychiat 1985; 142: $600-605$

342. Zubenko GS and Moossy J. Major depression in primary dementia: dinical and neuropathological correlates. Arch Neurol 1988; 45: 1182-1186.

343. Zubenko GS, Moossy J and Kopp U. Neurochemical correlates of major depression in primary dementia. Arch Neurol 1990; 47: 209-214. 
Diagnostic criteria for forgetfulness and mild dementia:

\section{Age-associated memory impairment (AAMI), Crook et al. , 1986 [59]}

Inclusion of:

1 Age above 50 years.

2 Complaints of memory loss reflected in everyday problems.

3 Onset of memory loss gradual, without sudden worsening in recent months.

4 Memory test performance at least 1 standard deviation below the mean established for young adults in a test of secondary memory with normative data.

5 Evidence of adequate intellectual function as determined on the Vocabulary subtest of the Wechsler Adult Intelligence Scale.

6 Absence of dementia as determined by a score of 24 or higher on the Mini-Mental State Exaination.

Exclusion of:

7 Evidence of delirium, confusion, or other disturbances of consciousness.

8 Any neurologic disorder that could produce cognitive deterioration.

9 History of infective or inflammatory brain disease.

10 Evidence of cerebral vascular pathology as determined by the Hachinski Ischaemia Score or by neuroradiologic examination.

11 History of repeated minor head injury or one major head injury.

12 Current psychiatric diagnosis (according to DSM-III criteria) of depression, mania or major psychiatric disorder.

13 Current diagnosis or history of alcoholism or drug dependence.

14 Evidence of depression as determined by the Hamilton Depression Rating Scale score of 13 or more.

15 Any medical disorder that could produce cognitive deterioration.

16 Use of any psychotropic substance or any other drug that may significantly affect cognitive function. 
Global deterioration scale (GDS), Reisberg et al. , 1982 [255]

Very mild cognitive decline (GDS 2: forgetfulness pbase):

Subjective complaints of memory deficits, most frequently in the following areas:

1 forgetting where one has placed familiar objects;

2 forgetting names one formerly knew well.

3 No objective evidence of memory deficits on clinical interview.

4 No objective deficits in employment or social situations. Appropriate concern with respect to symptomatology

Mild cognitive decline (GDS 3: early confusional phase):

Earliest clear-cut deficits.

Manifestations in more than one of the following areas:

1 patient may have got lost when travelling to an unfamiliar location

2 co-workers become aware of patient's relatively poor performance

3 word and name finding deficit become evident to intimates

4 patient may read a passage or a book and retain relatively little material

5 patient may demonstrate decreased facility in remembering names upon introduction to new people

6 patient may have lost or misplaced an object of value

7 concentration deficit maybe evident on clinical testing

And

8 Objective evidence of memory deficits obtained only with an intensive interview

9 Decreased performance in demanding employment and social settings

10 Denial begins to become manifest in patient. Mild to moderate anxiety accompanies symptoms. 
Clinical dementia rating (CDR) Hughes et al. 1982 [139]

Questionable dementia (CDR 0,5):

1 Mild consistent forgetfulness; partial recollection of events; "benign forgetfulness"

And no more than two of the following:

2 Some difficulty with time relationships; oriented for place and person at examination but may have geographic disorientation

3 Moderate difficulty in handling complex problems; social judgement usually maintained

4 Unable to function independently at community affairs though may still be engaged in some; may still appear normal in casual inspection

5 Mild but definite impairment of function at home; more difficult chores abandoned; more complicated hobbies and interests abandoned

6 Needs occasional prompting in personal care

Or:

1 No memory loss or slight inconstant forgetfulness

and two of the above mentioned criteria 2.6

Mild dementia (CDR I)

1 Moderate memory loss, more marked for recent events; defects interferes with everyday activities

At least three of the following:

2 Some difficulties with time relationships; oriented for place and person at examination but may have geographic disorientation

3 Moderate difficulty in handling complex problems; social judgement usually maintained

4 Unable to function independently in community affairs though still be engaged in some; may still appear normal to casual inspection

5 Mild but definite impairment of function at home; more difficult chores abandoned; more complicated hobbies and interests abandoned

6 Needs occasional prompting in personal care 


\section{CAMDEX Roth et al., 1986 [271, 272]}

\section{Minimal dementia:}

1 Limited and variable impairment in acquisition of new information and in recalling recent events

2 An increased tendency to misplace and lose possessions

3 Minor and variable errors in orientation

4 Some blunting in the capacity to follow or pursue a reasoned argument and to solve problems

5 Occasional errors (but of slowly advanced frequency) in occupational tasks and/or housework, Errors of judgement on occasion in professional or highly skilled tasks or socially responsible roles requiring difficult decisions or choices

6 Self-care unimpaired

7 Emotional life and responses well preserved

8 Clinical examination usually yields negative results except for manifest anxiety when asked to carry out demanding tasks

\section{Mild (early) dementia:}

1 Difficulty in acquiring new information and recalling recent events. Belongings are therefore lost or misplaced and information imparted intermittently forgotten or totally lost

2 Orientation as regards the date, day or week, time and place is impaired to a limited extent or in a patchy and inconsistent manner

3 Impairment is evident in activities demanding problemsolving or reasoning

4 speech shows mild defects in respect of clarity of meaning

5 Defects of knowledge of names of prominent figures, important events, simple geographical information

6 Impairment of skills in daily living, errors and confusion of tasks in everyday work, mistakes in housework, cooking (inappropriate ingredients or other errors). More conspicuous errors of judgement and inappropriate conduct in professional, highly skilled or socially responsible activities

7 Self care mildly impaired or not at all. There may be occasional errors in dress and a limited decline from usual standards of tidiness and cleanliness

8 Emotional responsiveness may be well retained or mildly impaired according to the type of dementia. There may be blunting or lability of emotion or both

9 Clinical examination at this stage shows the social facade well preserved, but systematic enquiry reveals indoubitable cognitive deficits and emotional or personality changes 
Items of six different sets of criteria for the diagnosis of ischaemic vascular dementia

Hachinski Rosen Loeb DSM-III Erkinjuntti ADDTC

$[117] \quad[269] \quad[192] \quad[7] \quad[83] \quad[46]$

$\begin{array}{lcccccc}\text { abrupt onset } & 2 & 2 & 2 & - & + & - \\ \text { stepwise deterioration } & 1 & 1 & - & + & - & - \\ \text { fluctuation } & 2 & - & - & - & - & - \\ \text { nocturnal confusion } & 1 & - & - & - & - & - \\ \text { preserved personality } & 1 & - & - & + & - & - \\ \text { depression } & 1 & - & - & - & - & - \\ \text { somatic complaints } & 1 & 1 & - & - & - & - \\ \text { emotional lability } & 1 & 1 & - & - & - & - \\ \text { hypertension } & 1 & 1 & - & - & - & - \\ \text { history of strokes } & 2 & 2 & 1 & + & - & + \\ \text { signs of ath.sclerosis } & 1 & - & - & - & - & - \\ \text { focal neurol. symptoms } & 2 & 2 & 2 & + & + & + \\ \text { focal neurol. signs } & 2 & 2 & 2 & + & + & + \\ \text { low density area on ct } & - & - & 2 / 3 \mathrm{a} & \pm \pm & + & + \\ \text { multiple strokes } & - & - & - & \pm \pm & + & +\mathrm{c} \\ \text { temporal relation } & - & - & - & - & +\mathrm{b} & +\mathrm{d}\end{array}$

note: $+=$ obligatory; $\pm \pm=$ ambiguous

a either isolated ( 2 points) or multiple ( 3 points) hypodense areas on ct scan

$b$ in absence of a temporal relation: probable vd

$c$ at least one infarct outside the cerebellum

$d$ in case of a single stroke 


\section{Guidelines for the rating of awareness of cognitive deficits}

General instructions:

After the clinician has introduced himself, he concentrates on the patient, and the caregiver is requested to refrain from any comment. The following questions are asked: Please tell me about the problems you are bere for. Wby did dr... send you to this clinic? When the patient has other complaints not directly related to dementia: Do you bave any otber complaints?

When the patient has no spontaneous complaints about his cognitive functions: How is your memory functioning? Do you think you bave a poor memory? When the patient denies deficits of memory or other cognitive functions: So, there are no memory problems at all? Is everytbing going all rigbt for you? After these opening questions, the complaints are discussed more extensively in an open interview, in which the clinician tries to get an impression of the degree and the nature of the cognitive symptoms and the patient is asked to provide examples.

Then, the clinician puts the same questions to the caregiver adapted to find out about the patients' cognitive functions (e.g., Wby did dr... referred your fatber/motber/... to this clinic?). Scoring of awareness is made directly after the interview. Allowance should be made in scoring for cognitive impairments such as paraphasias or concretisms (eg, "There is a bole in my brain").

\section{Scoring}

4 Adequate

3 Mildly disturbed

2 Moderately disturbed

1 Severely disturbed
Patient has adequate knowledge of his cognitive deficits. Spontaneous complaints about memory or other cognitive disfunctions. Gives examples. History of the patient is congruent with the history of the informant.

Patient has some knowledge of his cognitive deficits, but with some gaps. Spontaneous complaints about memory. History of the patient shows some discrepancies with the history of the informant.

Patient has only vague and passive knowledge of cognitive deficits. No spontaneous complaints, admits to memory deficits only when questioned about them. Obvious discrepancies with the history of the informant.

Denies any deficits. No complaints about memory whatsoever, even after explicit questioning. 


\section{Dankwoord}

Voordat één lettertje geschrapt kon worden uit mijn academische titel (drs), moesten er eerst 421.970 letters geschreven worden. Dit had niet kunnen gebeuren zonder de steun van velen. Bij het voltooien van dit proefschrift wil ik de kans aangrijpen allen te bedanken die hieraan hebben bijgedragen. Een aantal wil ik bij naam noemen.

Professor Jolles, beste Jelle. Jou ben ik met name zeer erkentelijk voor je voortdurende stimulans, je inspirerende en enthousiasmerende ideeën en de ruimte die je mij altijd gegeven hebt. Ik hoop dat onze altijd plezierige en vriendschappelijke samenwerking nog lang kan worden voortgezet.

Professor van Praag, beste Herman. Ik dank je voor je de snelle en kritische wijze, waarop je eerdere versies van het manuscript van commentaar voorzag. Ik voel het als een eer een steentje aan jouw 'laatste berg' bij te dragen.

Zeer veel dank gaat uit naar Nico Rozendaal voor zijn snelle onvermoeibare ondersteuning bij het analyseren van de data en voor zijn waardevolle methodologische bijdragen. Ik zou alle promovendi toewensen dat zij met een even kundig en sympatiek iemand kunnen samenwerken.

Mijn naaste collegae, met name Rudolf Ponds, Fred Vreeling Leo Plugge en Peter Houx, wil ik danken voor de plezierige en collegiale wijze waarop wij met elkaar van ideeën wisselden en de Wetenschap bespraken. Jullie vormen een goed bewijs voor de stelling dat de beste basis voor een wetenschappelijke samenwerking gevormd word door een goede onderlinge verstandhouding. De studenten Juliette Nijlant, Liesbeth Hak, Ingrid Bayens en Liesbeth Scheepers zeg ik dank voor hun hulp bii het verzamelen van gegevens voor dit proefschrift. 
Professor van der Lugt en professor Troost ben ik zeer erkentelijk voor de faciliterende steun om daadwerkelijk te komen tot een geïntegreerde neurologisch-psychiatrische voorziening als de Maastrichtse geheugenpoli. Ook professor Verhey ben ik dankbaar voor zijn medewerking in dezen, maar het zal duidelijk zijn dat ik hem uiteraard voor nog veel meer in mijn leven erkentelijk ben.

Ook wil ik Jane Sykes-Bär en Ann Knip-Hilton hier bedanken voor de nauwgezette wijze waarop zij van mijn inglisjh English maakten.

Alle andere collegae die dagelijks ervoor zorg(d)en dat de geheugenpolikliniek prettig is om in te werken is ben ik eveneens dankbaar: Helma de Veth-Servais, Maria Braam, Rinie Lenaerts, Monique de Lugt, Christel Bruggeman, Robbie Coppens, Rianne wennekes, Anita Hendriks, Aggie Savelkoul, Charlotte Kindt.

Maar het meest nog dank ik Brigit. En Erik, Josefien en Louis. 


\section{Curriculum Vitae}

Frans Verhey werd op 9 november 1955 geboren te Maastricht. Na het gymnasium bèta in Maastricht studeerde hij geneeskunde aan de Universiteit van Amsterdam. Na zijn doctoraal examen (1978) volgde hij een stage in Caçapava do Sul, Rio Grande, Brazilië bij dr. Victor Lang. In 1980 was hij zes maanden als assistent werkzaam op de afdeling cardiologie in het voormalige Binnen-Gasthuis bij prof, dr. A. J. Dunning. In 1982 begon hij zijn specialisatie als zenuw- en zielskundige (zenuwarts): zijn stage neurologie werd gevolgd in het ziekenhuis St Annadal te Maastricht bij prof. dr. P.J.M. van der Lugt, zijn stage psychiatrie deed hij in het Academisch Ziekenhuis Utrecht bij prof. dr. M. Kuilman. Het viffde keuzejaar was gewijd aan de neuropsychologie en wetenschappelijk onderzoek, onder leiding van prof. dr. J. Jolles, Rijksuniversiteit Limburg te Maastricht. Op 1 april 1987 werd hij ingeschreven in het specialistenregister. Vanaf 1986 was hij werkzaam als universitair docent bij de vakgroep Neuropsychologie \& Psychologie. Vanaf 1993 is hij staflid / hoofd polikliniek psychiatrie in het Academisch Ziekenhuis Maastricht (hoofd: prof. dr. H. M. van Praag). Hij is medisch hoofd van de Maastrichtse Geheugenpolikliniek en consulent neurologie/neuropsychiatrie in het Psychomedisch Streekcentrum Vijverdal. Zijn huidige wetenschappelijke activiteiten hebben betrekking op de vroege onderkenning van dementie, diagnostiek en behandeling van organisch affectieve stoornissen, de toepasbaarheid van brain-imaging technieken bij neuropsychiatrische patiënten en klinisch geneesmiddelen onderzoek bij de ziekte van Alzheimer. Hij is getrauwd met Brigit Hoek; zij hebben drie kinderen: Erik, Josefien en Louis. 


\section{List of Publications}

Bohnen, N., Jolles, J. and Verbey, F. R. J. (1993) Persisitent neuropsychological deficits in cervical whiplash patients without direct headstrike. Acta neurologica Belgica 93, 23-31.

Commissaris, C. J. A. M., Verbey, F. R. J. , Ponds, R. W. H. M., Jolles, J. and Kok, G.J. (1993) Publieksvoorlichting over normale vergeetachtigheid en dementie. Effectiviteit van een planmatig ontwikkelde voorlichtingsbrochure. Tijdsch Geront Geriat, in press

Commissaris, C. J. A. M., Jolles, J., Ponds, R. W. H. M., Verbey, F. R. J., Damoiseaux, V. and Kok, G. J. (1993) Vergeetachtig of dement? wie maakt zich zorgen en waarom? Tijdsch Geront Geriat, in press

Commissaris, C. J. A. M., Jolles, J., Reyersen van Buuren, E. J. and Verbey, F. R. J. (1993) Effecten van geheugenvoorlichting door middel van functiegerichte begeleiding (FGB). Gedrag en Gezondheid.

Commissaris, C. J. A. M., Verbey, F. R.J., Ponds, R. W. H. M., Jolles, J. and Kok, G. J. (1993) Determinanten van ervaren problemen van thuisverzorgende partners van dementie-patienten. Gedrag en Gezondheid 21, $17-25$.

Commissaris, C. J. A. M., Verbey, F. R. J., Ponds, R. W. H. M., Jolles, J. and Kok, G.J. (1993) Publiekswoorlichting over normale vergeetachtigheid en dementie. Belangen en effecten. Tijdschrift voor Sociale Gezondheidszorg 71(3236).

Jolles, J., Verbey, F. R.J. and Bohnen, N. (1989) Veranderingen in het centrale zenuwstelsel bij veroudering en de gevolgen hiervan op de urine-productie. Nederlands Tijdschrift voor Geneeskunde 113, 1811-1812.

Jolles, J. and Verhey, F. R. J. (1993) On the rationale behind treatment studies in cognitive aging and dementia. In Alzheimer's disease: back to the future (E. C. Wolters and P. Scheltens, Eds). Vrije Universiteit Amsterdam, Amsterdam.

Jolles, J., Houx, P. J., Vreeling, F. W. and Verbey, F. R. J. (1993) Cognitive aging, biological life events and primitive reflexes. Neurosci Lett, in press.

Jolles, J, Verhey, F. R. J., Houx, P. J. and Reyersen van Buuren, E. J. (submitted) Cognitive dysunctions in middleaged subjects with late-onset dysthymia.

Plugge, L. A., Verhey, F. R.J. and Jolles, J. (1988) Het EVINCE-project: expertsysteemontwikkeling door dementiedifferentiaaldiagnostiek. De Psycholoog 23(9), 497. 
Plugge, L. A., Verbey, R.RJ. and Jolles, J. (1989) Een experimenteel expertsysteern voor differentiële diagnostiek van het dementiesyndroom. TijdschGeront Geriat 20,51-58.

Plugge, L. A, Verhey, F.RJ. and Jolles, J. (1989) Experimental Expert System for Differential Diagnosis of Dementia. DIGEST: Parkinson/Azheimer 8, 26-27.

Plugge, L. A., Verhey, F. R J. and Jolles, J. (1990) Desk-Top expert system for the differential diagnosis of dementia: An evaluation study. Int J Technol Ass 6, 147-156.

Plugge, L. A, Verbey, F. R. J. and Jolles, J. (1990) Evince: een expertsysteem voor dementiediagnostiek. Klein symposium Automatisering en Diagnostiek in de psychogeriatrie, Maastricht, 19-01-1990. Tijdsch Geront Geriat 21, $133-135$.

Plugge, L. A, Verhey, F.RJ. and Jolles, J. (1990) EVINCE: een expertsysteem voor dementiediagnostiek. Tijdsch Geront Geriat 21, 135 .

Plugge, L. A., Verbey, F. R.J. and Jolles, J. (1991) Differential diagnosis of dementia: a comparison between the expert system EVINCE and clinicians. Joumal of Neuropsychiatry and dinincal neurosciences 3, 398-404.

Plugge, L., Verhey, FR. J., Everdingen, v.E. and Jolles, J. (1991) Differential diagnosis of dementia. An experimental study into intra-and interdisciplinary agreement. J Geriatric Psychiat Neurol 4, $90-97$.

Ponds, R. W. H. M. Verhey, F. RJ., Rozendaal, N., Jolles,J. and Deelman, B. G. (1992) Dementiescreening: validiteit van de cognitive screeningstest en de mini-mental state examination. T Geront Geriat 23, 94-99.

Ponds, R. W. H. M., Verbey, F. R J, Rozendaal, N., Jolles, J. and Deelman, B. G. (1992) Brief cognitive screening tests for dementia: comparison of the mini-mental status examination and the cognitive screening test. In Gerontechnology (Studies in Health Technology and Informatics) (H. Bouma and J A. M. Graafmans, Eds). IOS Press, Amsterdam.

Scheltens, P. Verhey, F. R.J, Boer, M. d. and Wolters, E. C. (1991) Langzaam progressieve afasie zonder dementie. Ned Tijdschr Geneesk 135, 898-901.

Verhey, F. R.J., Reyersen van Buuren, E. and Jolles, J. (1987) De geheugenkliniek: multidisciplinaire benadering bij stoornissen van het geheugen en andere cognitieve stoornissen. Ouder worden Nu (Houweling. T. J, Eds), Gerontologisch instituut, Amsterdam.

Verbey, F.RJ and Jolles,J. (1988) Over de spraakverwarring rond het begrip dementie en de zickte van Alzheimer. Tijdsch Geront Geriat 19,89.96.

Verhey, F. R. J. Vreeling, F. W. and Jolles, J. (1989) DSM-III and NINCDS/ADRDA criteria for dementia and Alzheimer's disease: impact of diagnostic procedures on dally practice. In Fifth Meeting of the Intemational Study Group on the pharmacology of Memory Disorders Associated with Aging, Zürich, january 1989 (J. Wurtman, Eds), Zürich. Center for Brain Sciences and Metabolism Charitable Trust. 
Verbey, F. R. J. (1989) Consensus conference on the methodology of clinical trails of Noötropics. Congresverslag, München 28-30 juni 1989. Tijdsch Geront Geriat 20, 215-216.

Verhey, F. R J.; Ponds, R. W. H. M., Jolles, J. and Lugt, P. J. M. v. d. (1991) Een fobie voor vergeten: een nieuw psychogeriatrisch syndroom. Medisch Contact 46, 575-576.

Verhey, F. R J. (1991) Post-Whiplash syndroom (ingezonden brief). Ned Tijdschr Geneesk 135, 678.

Verhey, F. R. J., Plugge, L. A., Everdingen, J. J. E. v. and Jolles, J. (1991) Verschillende disciplines, verschillende diagnosen? - een enquête onder de deelnemers van de consensusvergadering over dementie. Tijdsch Geront Geriat 22, 187-194.

Verbey, F. R J., Vreeling, F. W. and Jolles, J. (1992) Dementie bij amyotrofische laterale sclerose. Acta Neuropsychiat, 18-21.

Verhey, F. R. J., Ponds, R. W. H. M., Jolles, J. and Commissaris, C. J. A. M. (1992) Vergeetachtig? of Dement? Alzheimer Stichting, Bunnik.

Verbey, F. R. J., Jolles, J., Ponds, R. W. H. M., Rozendaal, N., Plugge, L., Vet, H. C. W. d. et al. (1993) Diagnosing dementia: a comparison between a monodisciplinary and multidiscipinary approach. J Neuropsychiat Clin Neurosc 5 ,
$78-85$.

Verbey, F. R. J., Roozendaal, N., Ponds, R. W. H. M. and Jolles, J. (1993) Dementia, depression and awareness. IntJ Geriat Psychiat. in press

Verbey, F. R. J. Jolles, J., Ponds, R. W. H. M. and Vreeling, F. W. (1993) Psychiatrische stoomissen bij patienten van een geheugenpolikliniek. Ned Tijdschr Geneesk 137, 1054-1058.

Verhey, F. R. J., Rozendaal, N., Ponds, R. W. H. W. and Jolles, J. (1993) Depression and insight in Alzheimer's disease and vascular dementia. In Alzheimer's Disease and Related Disorders (M. Nicolini, Eds). Pergamon Press Ltd,
Oxford, $85-86$

Vreeling, $\mathrm{F}$. W. Jolles, J., Verhey, F. RJ. and Houx, P.J. Primitive reflexes in healthy, adult volunteers and neurological patients: Methodological issues. J Neurol in press

Vreeling, F. W. Verhey, F. RJ., Houx, P. J and Jolles, J. Primitive reflexes in Parkinson's diseasse.J Neurol Neurosurg Psychiat, in press

Vreeling, F. W., Jolles, J., Verhey, F. R. J. and Houx, P. J. Primitive reflexes in Alzheimer's disease and vascular dementia. J Geriatric Psychiat Neurol, in press

Vreeling, F, W., Jolles, J, Verbey, F. R J J and Houx, P. Prinitive reflexes in healthy, adult volunteers and neurological patients: Methodological issues. J Neurol, in press 
Submitted:

Verhey, F. R J., Houx, P. Ponds, RWHM., Rozendaal, N. and Jolles, J. (1993) Two year follow-up of non demented patients from a memory dinic.

Verhey, F. R.J., Lodder, J., Rozendaal, N. and Jolles, J. (1993) A comparison between six current criteria for the diagnosis of Vascular Dementia.

Verbey, F. R.J., Ponds, RWYHM., Rozendaal, N. and Jolles, J. (1993) Depression, insight and personality changes in Alzheimer's disease and vascular dementia.

Vreeling, F. W., Jolles, J,, Houx, P.J. and Verhey, F. R J. Primitive reflexes in age-associated memory impaiment.

Published abstracts:

Jolles,J,, Reyersen van Buuren, E. J., Houx, P.J., Plugge, L.A and Verbey, F. R.J. (1990) Early diagnosis of Alzheimer's disease and the differentiation from normal aging. Eur $\mathrm{Clin}$ Invest 20(2-I), A61.

Plugge, L. A, Verhey, F. R. and Jolles,J. (1990) The dementia diagnostic expert system Evince in comparison with expert clinicians: an evaluation study. EurJ Clin Invest 20(2), A62.

Plugge, L. A, Verhey, F. R. J. and Jolles, J. (1990) Evaluation of an expert system for dementia diagnostics (EVINCE): expert system performs better than clinicians. Neurobiol Aging 11(3), 255-256.

Reyersen van Buuren, E.J., Verbey, F. R.J. and Jolles, J. (1987) Contributions for neuropsychology to early diagnosis of demential syndromes and the implication for treatment and care. Clin Neurol Neurosurg 89(suppl. II), 22-23.

Verhey, F. R.J., Reyersen van Buuren, E. J. and Jolles, J. (1987) Neuropsychiatric disturbances in the presenium: possible contribution to early diagnosis of dementia. Clin Neurol Neurosurg 89 supl II, 22.

Verhey, F. RJ., Reyersen van Buuren, E.J., Vreeling, F. W. and Jolles, J. (1988) Necessity of a multidisciplinary and Systematic diagnostic model in early and differential diagnosis of demential syndromes. In international symposium on Atheimer's Disease, Kuopio, Finland.

Verhey, F. R.J., Plugge, L. A and Jolles, J. (1989) Dementa and Alzheimer's disease: factors influencing the diagnosis. Eur J Clin Invest 20(2), A61.

Verbey, F. R. J., Ponds, R. W. H. M., Reyersen van Burten, E. J., Vreeling, F. W. and Jolles, J. (1990) Application of research criteria for dementia in common clinical practice. Neurobiol Aging 11(3), 298-299.

Vreeling, F. W. Verbey, F. R. J., Howx, P. and Jolles, J. (1988) Methodological problems in the assessment of neurological signs, i.e. primitive reflexes, in aging and dementia. In 2nd International Symposium on Alzheimers Diseases, Kuopio, Finland. 
